\title{
Particulate Deposition Prediction of Diluted Two-Phase Impinging Jet
}

\author{
By
}

\section{Eid Alatawi}

A thesis submitted to the Faculty of Graduate and Postdoctoral Affairs in partial fulfilment of the requirements for the degree of

\section{Doctor of Philosophy}

\section{in Mechanical Engineering}

Ottawa-Carleton Institute for Mechanical and Aerospace Engineering

Department of Mechanical and Aerospace Engineering

Carleton University

Ottawa, Ontario, Canada

July 2013

(C) 2013, Eid Alatawi 


\section{Abstract}

Insights into particle transport and deposition process in impinging jet flow, in light of the available experiments, can be gained using computational fluid dynamics (CFD) numerical simulations. The present thesis focuses mainly on the prediction of aerosol particle transport and deposition in impinging jet flow. An extensive literature survey has indicated that the present work represents the first comprehensive investigation of aerosol particle transport and deposition, using Reynolds averaged Navier Stokes/eddy interaction model (RANS/EIM) along with near-wall corrections, and large eddy simulation (LES) numerical approaches applied to particle-laden impinging jet flow.

A new in-house tracking code for particle-laden impinging jet flow using modified EIM, as well as modified EIM in conjunction with the near-wall correction technique based on impinging jet flow characteristics was developed to simulate the particulate phase. Two different approaches in the framework of RANS method, RANS SST (shear stress transport) and RANS RSM-BSL (Reynolds stress transport-Baseline) model were used to simulate the fluid phase in three cases of nozzle-to-surface distances of $L / D=2,4$ and 6. Also, to better understand the applicability and accuracy limits of different numerical methods on aerosol particle deposition, one representative case for an impinging jet flow of $L / D=2$ was performed using LES.

Deposition results without near-wall correction, with turbulent tracking, showed unrealistic behavior at the beginning of the wall jet region and close to the stagnation point. Once the normal-to-wall fluctuating velocity, which plays important role for particle deposition on the impingement wall, was properly modeled via the near-wall 
correction technique, significant improvements were obtained when compared to the previous experiments, for all $L / D$ cases. However, the results showed that RANS RSMBSL/modified EIM, in conjunction with the near-wall correction, have better performance in predicting the deposition results. Particle deposition results for $L / D=2$ showed that LES is in closer agreement with previous experimental data more than RANS RSM-BSL/modified EIM along with near-wall correction. These results provide new insight into the general behavior of the aerosol particle transport and deposition process in impinging jet flow. 


\section{Acknowledgements}

All praises and glory to Almighty ALLAH the exalted who granted me the support, courage, and patience during my PhD study. I thank ALLAH for giving me supreme opportunities and the strength to rise to the challenge of treating the difficult tasks in my life. I thank him for helping me complete this work.

I want to express sincere appreciation to my thesis supervisor, Dr. Edgar Matida, who offered me valuable guidance, advice and encouragement during my research, and also in my career life. Working with him has been very interesting and beneficial. Also, I want to thank my colleagues for their assistance while I conduct this research. I am also grateful to all academic members of the department for their assistance. I want to acknowledge in particular Neil, Christie, Irene and Nancy.

My deepest appreciation goes to my parents for their patience, endless encouragement and everlasting love. My deepest gratitude goes to my wife for her sacrifice and unconditional support that allowed me to follow a dream. Finally, I want to acknowledge King Abdulaziz City for Science and Technology that sponsored me during this research. 


\section{Table of Contents}

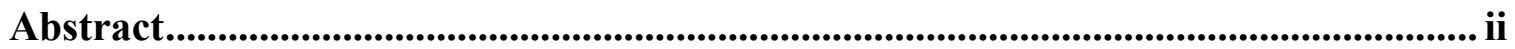

Acknowledgements ............................................................................................................... iv

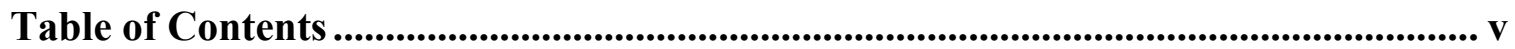

List of Tables .......................................................................................................................... viii

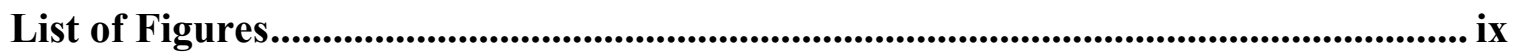

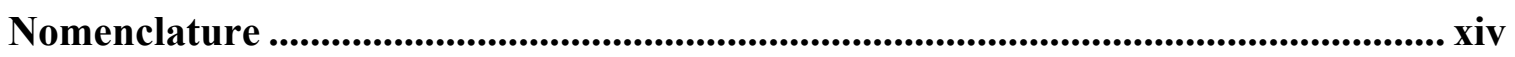

Chapter 1: Introduction ........................................................................................................ 1

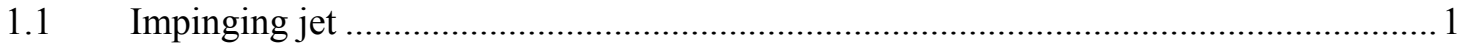

1.2 Two-phase interactions and deposition mechanisms................................................... 5

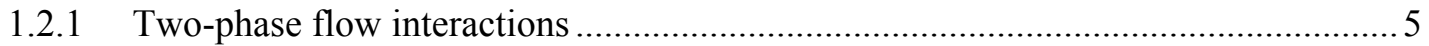

1.2.2 Deposition mechanisms ..............................................................................

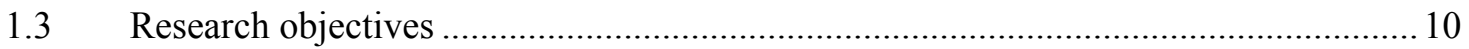

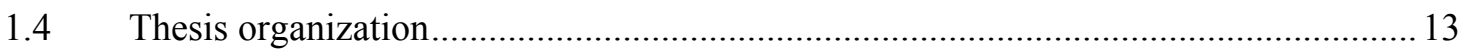

Chapter 2: Literature survey .................................................................................................. 14

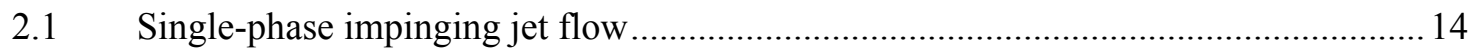

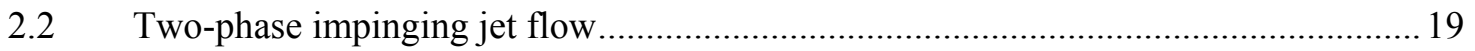

2.3 Previous studies of different particle-laden flows .......................................................2

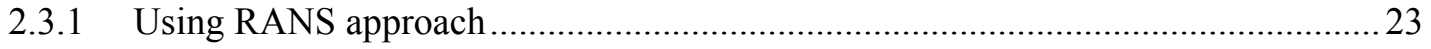

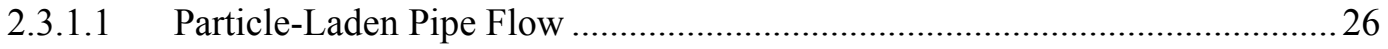

2.3.1.2 Particle-Laden Free Jet Flow ......................................................................... 32

2.3.2 Using LES approach ........................................................................................ 34

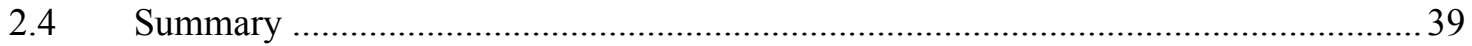

Chapter 3: Numerical Methods .......................................................................................... 40

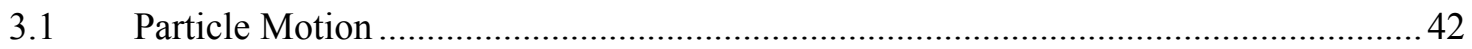

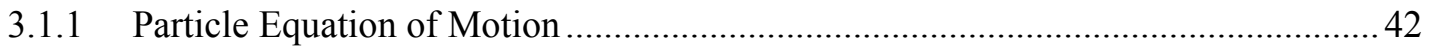

3.2 Reynolds Averaged Navier Stokes (RANS) Equations.................................................. 44

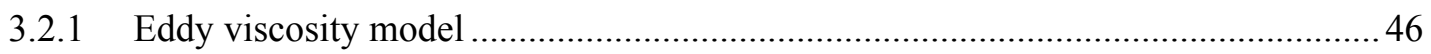

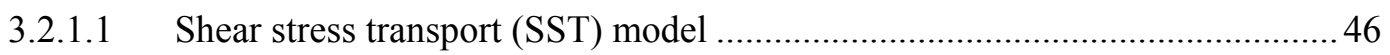

3.2.2 Reynolds stress model (RSM) ..................................................................... 47

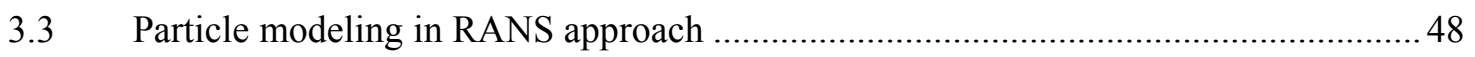

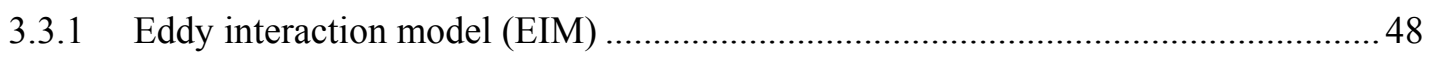




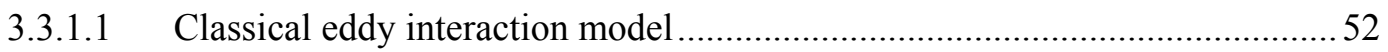

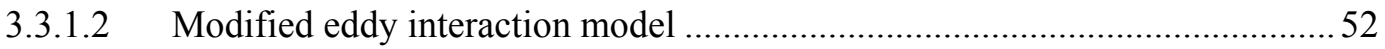

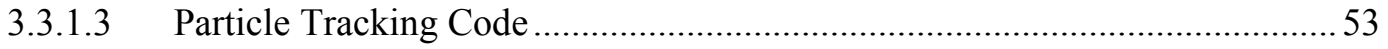

3.3.1.4 Details of exporting the flow field and connectivity data................................56

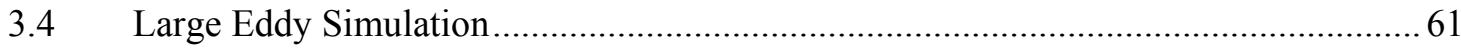

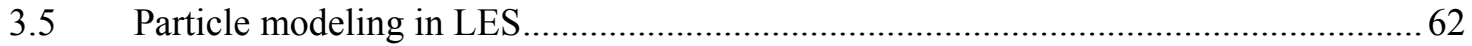

Chapter 4: Results for RANS-SST with EIM ....................................................... 64

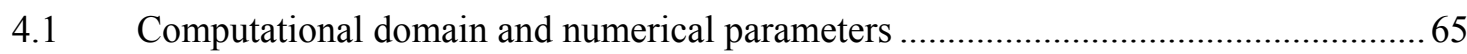

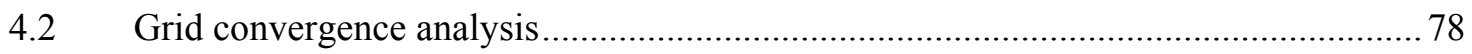

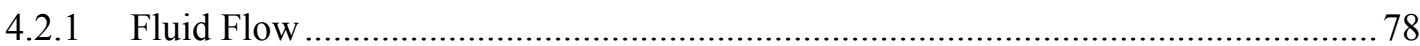

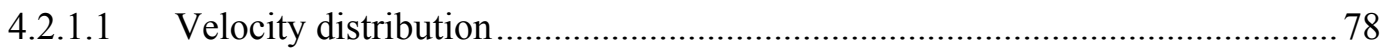

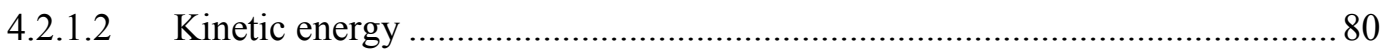

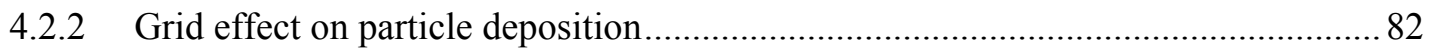

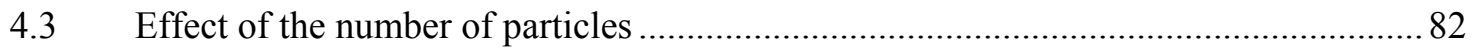

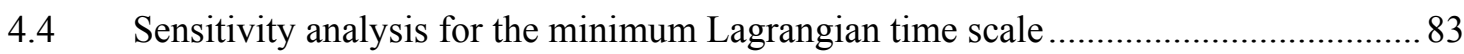

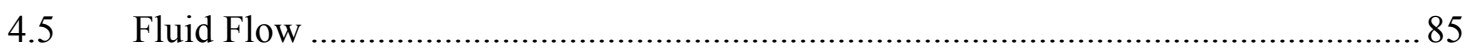

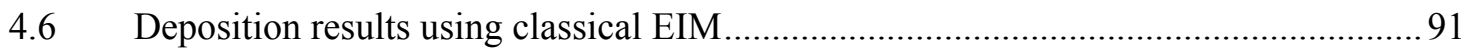

4.6.1 Turbulent tracking and mean flow tracking ......................................................... 91

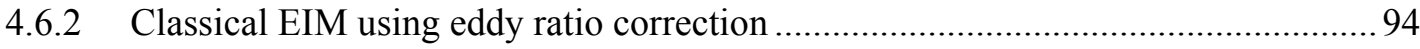

4.7 Particle distribution over the impingement surface …........................................... 97

4.8 Effect of the nozzle-to-surface distance on particle deposition ................................. 101

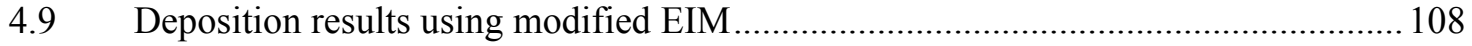

4.9.1 Turbulent tracking and mean flow tracking ....................................................... 108

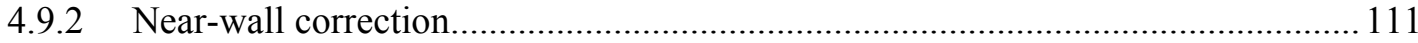

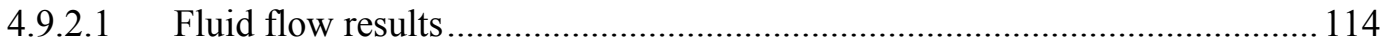

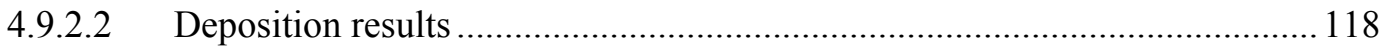

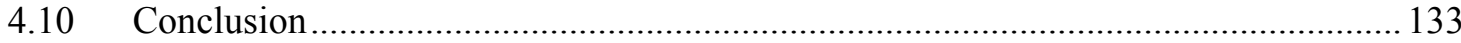

Chapter 5: Results for RANS-RSM with EIM....................................................... 135

$5.1 \quad$ Computational domain and numerical parameters ................................................. 135

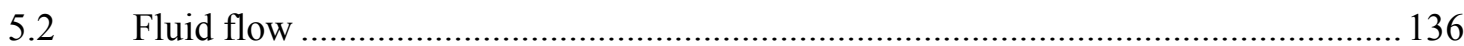

5.3 Deposition results using modified EIM................................................................. 144

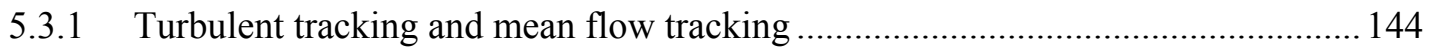

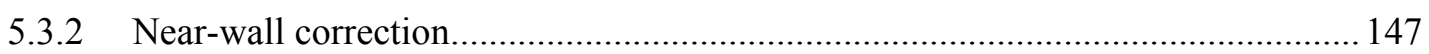

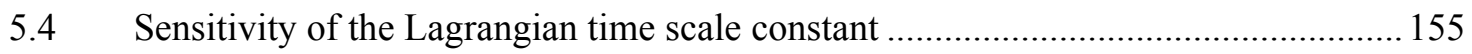




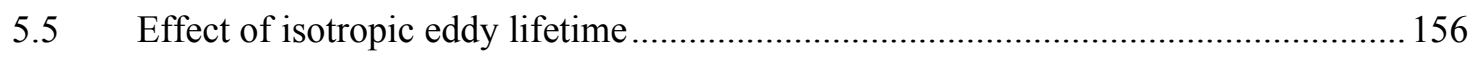

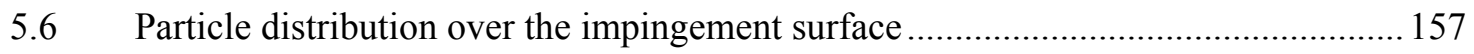

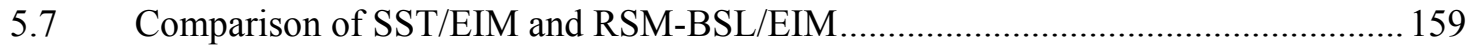

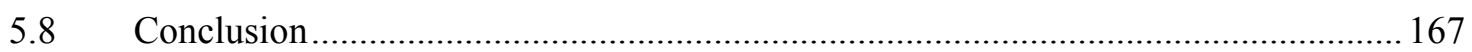

Chapter 6: Results Using LES ............................................................................ 168

6.1 Computational domain and numerical parameters ................................................ 169

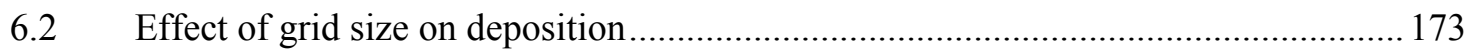

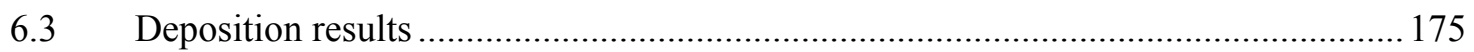

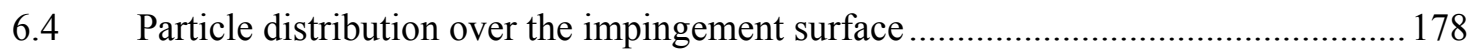

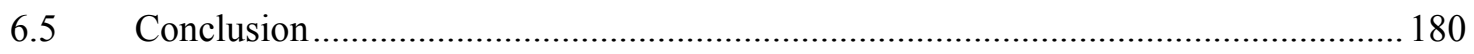

Chapter 7: Conclusions and Future Work ............................................................ 181

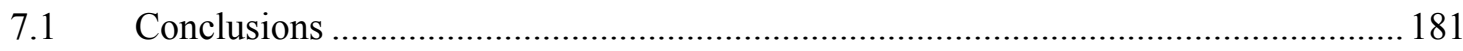

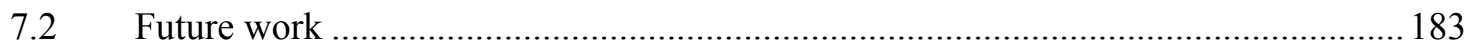

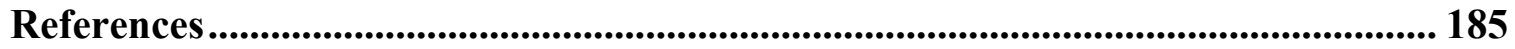

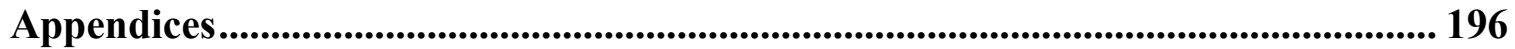

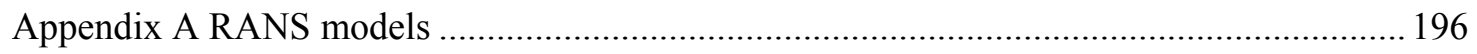

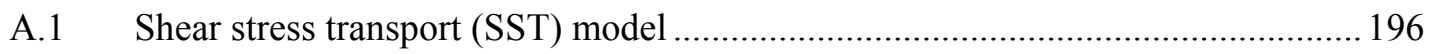

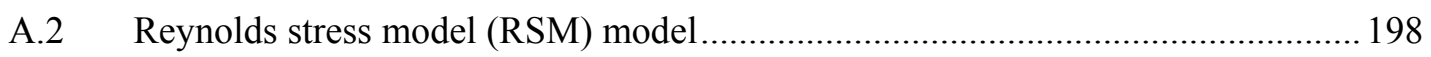

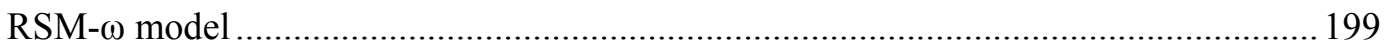

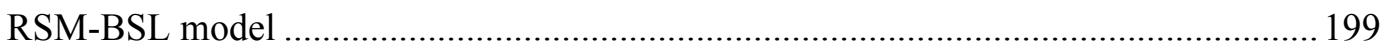

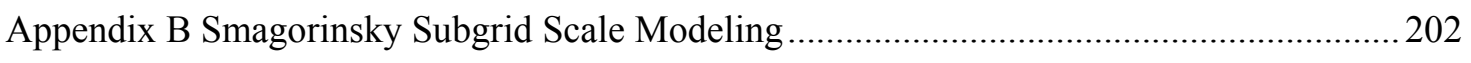

Appendix C Grid convergence for computational domains based on Cooper's experiment ...204

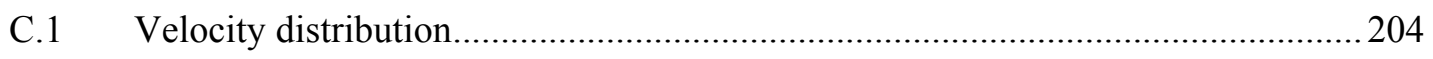

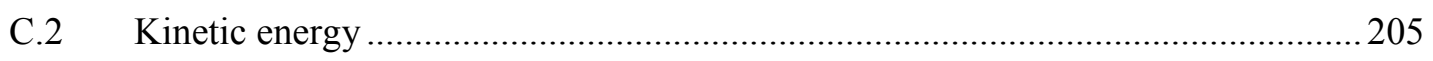




\section{List of Tables}

Table 1.1. Differences between the impinging jet and the flow parallel to walls (Cooper et

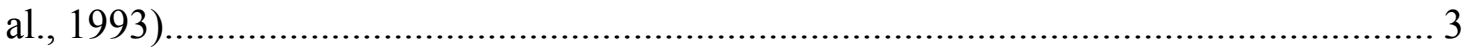

Table 2.1. Summary of previous studies on single-phase impinging jet flow................ 15

Table 3.1. Connectivity of block 1 with other blocks and boundaries. .......................... 59

Table 4.1. Details of computational domains. ................................................................ 70

Table 4.2. Details of computational domains based on Cooper's experiment. ................. 77 


\section{List of Figures}

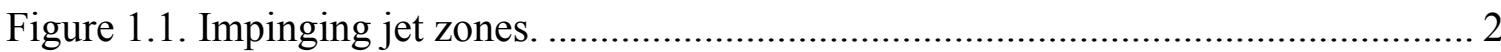

Figure 1.2. Map of regimes of fluid-particle interaction (adopted from Elghobashi, 1994).

Figure 2.1. Normal-to-wall RMS fluctuatuating velocities for $\mathrm{L} / \mathrm{D}=2$ and 6 . (Cooper et al., 1993). 18

Figure 2.2. Impaction pattern. (adopted from Sethi and John, 1993) ............................. 21

Figure 2.3. Deposition behaviour in impinging jet. (Burwash et al., 2006).................... 23

Figure 2.4. Particle deposition velocity versus nondimensional particle relaxation time.

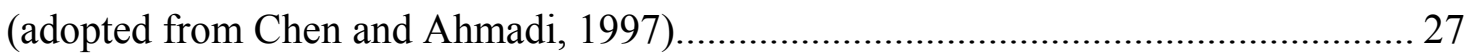

Figure 2.5. Deposition efficiency in turbulent bend flow (adopted from Breuer et al., 2006). 37

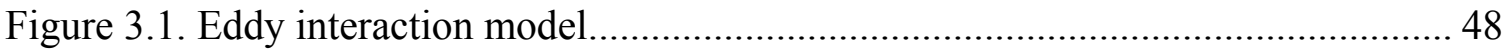

Figure 3.2. Transformation of the hexahedric element: (a) in $x y z$ system, and (b) in $a b c$

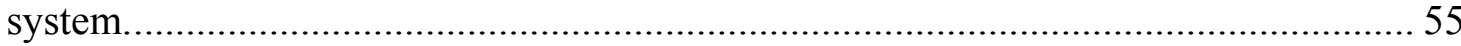

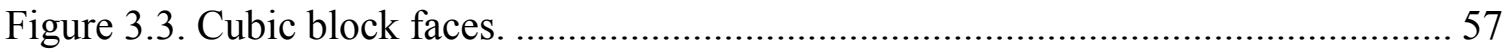

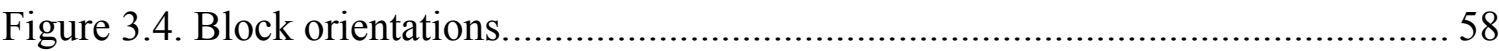

Figure 3.5. Flow chart of the steps of exporting the flow field and connectivity information.

Figure 4.1. Schematic of the computational domain of the impinging jet based on the

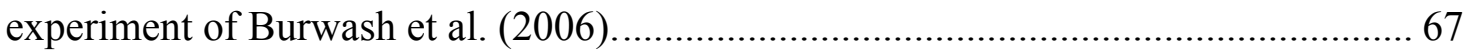

Figure 4.2. Grid of the computational domain of the impinging jet used for RANS/EIM calculations for: (a) Top view of the computational domain, and (b) Zoom of the normal-to-wall mesh in $\mathrm{x}-\mathrm{z}$ plane $(\mathrm{y}=0)$.

Figure 4.3. Schematic of the computational domain of the impinging jet based on the experiment of Cooper et al. (1993).

Figure 4.4. Grid of the computational domain of the impinging jet used for RANS calculations based on Cooper et al.'s experiment for: (a) Top view of the computational domain, and (b) Zoom of the normal-to-wall mesh in $x-y$ plane................................ 75

Figure 4.5. Velocity magnitude along the centerline axis. ................................................ 79 
Figure 4.6. Velocity magnitude along a line parallel to the impingement wall $(z=0.1 \mathrm{D})$.

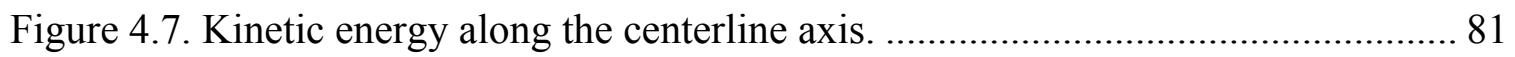

Figure 4.8. Kinetic energy profiles along a line parallel to the impingement ................. 81

Figure 4.9. Effect of grid resolution on the particle deposition................................... 82

Figure 4.10. Effect of number of particles on the deposition. .................................... 83

Figure 4.11. Sensitivity analysis for the minimum Lagrangian time scale..................... 84

Figure 4.12. Mean velocity magnitude (a-c), and normal-to-wall fluctuation (d-f) for L/D

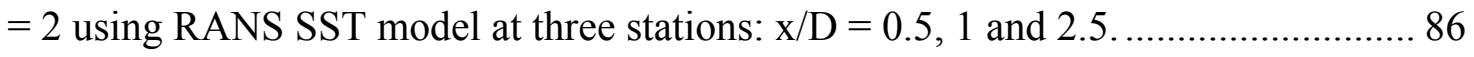

Figure 4.13. Mean velocity magnitude (a-c), and normal-to-wall fluctuation (d-f) for L/D

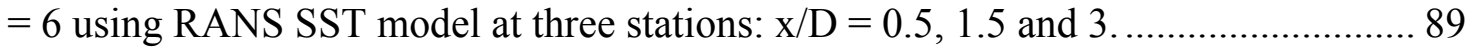

Figure 4.14. Deposition density using turbulent tracking and mean flow tracking for L/D

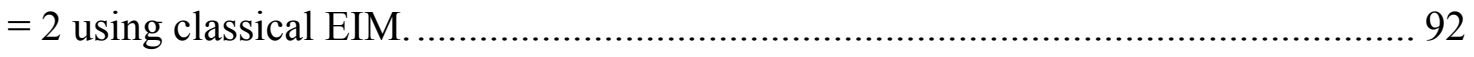

Figure 4.15. Deposition density using turbulent tracking and mean flow tracking for $\mathrm{L} / \mathrm{D}$

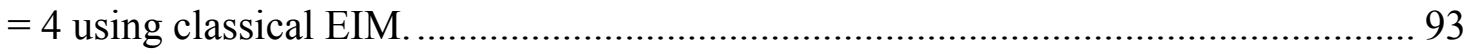

Figure 4.16. Deposition density using turbulent tracking and mean flow tracking for L/D

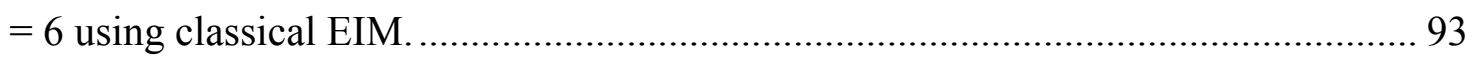

Figure 4.17. Effect of eddy viscosity correction on deposition density for $\mathrm{L} / \mathrm{D}=2 \ldots \ldots .95$

Figure 4.18. Effect of eddy viscosity correction on deposition density for $L / D=4$...... 96

Figure 4.19. Effect of eddy viscosity correction on deposition density for $L / D=6 \ldots \ldots .96$

Figure 4.20. The experimental deposition pattern for $\mathrm{L} / \mathrm{D}=2$. (Burwash et al., 2006)... 99

Figure 4.21. Particle deposition patterns for $\mathrm{L} / \mathrm{D}=2,4$ and 6 plotted for: $(\mathrm{a}, \mathrm{b}, \mathrm{c})$ turbulent

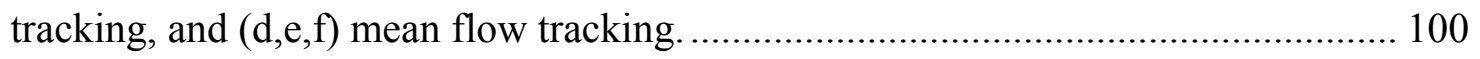

Figure 4.22. Particle deposition patterns plotted for $\mathrm{L} / \mathrm{D}=2,4$ and 6 ....................... 101

Figure 4.23. Centerline velocity along the jet axis for $\mathrm{L} / \mathrm{D}=2,4$ and 6 .................... 102

Figure 4.24. Turbulence kinetic energy along a line $(z=0.1 D)$ perpendicular to the jet axis for $\mathrm{L} / \mathrm{D}=2,4$ and 6 . 103

Figure 4.25. Turbulence kinetic energy at distances from the jet axis: (a) $x=0 D$, (b) $x=$ $0.5 \mathrm{D},(\mathrm{c}) \mathrm{x}=1.0 \mathrm{D}$, and $(\mathrm{d}) \mathrm{x}=2.0 \mathrm{D}$ for $\mathrm{L} / \mathrm{D}=2,4$ and 6 . 106

Figure 4.26. Contours ( $\mathrm{x}-\mathrm{z}$ plane, $\mathrm{y}=0)$ of the magnitude of the mean velocity $(\mathrm{a}-\mathrm{c})$, and turbulence kinetic energy (d-f) for $\mathrm{L} / \mathrm{D}=2,4$ and 6 . 107 
Figure 4.27. Deposition density using turbulent tracking (modified EIM) and mean flow tracking for $\mathrm{L} / \mathrm{D}=2$.

Figure 4.28. Deposition density using turbulent tracking (modified EIM) and mean flow tracking for $\mathrm{L} / \mathrm{D}=4$. 110

Figure 4.29. Deposition density using turbulent tracking (modified EIM) and mean flow tracking for $\mathrm{L} / \mathrm{D}=6$. 110

Figure 4.30. Mean velocity magnitude (a-c), and normal-to-wall RMS (d-f) for L/D = 2 at three stations: $\mathrm{x} / \mathrm{D}=0.5,1$ and 2.5 . 115

Figure 4.31. Mean velocity magnitude (a-c), and normal-to-wall RMS fluctuation (d-f) for $\mathrm{L} / \mathrm{D}=6$ at three stations: $\mathrm{x} / \mathrm{D}=0.5,1.5$ and 3 . 116

Figure 4.32. Calibrating normal-to-wall RMS fluctuation using SST model without Kato and Launder modification (a-c), and with Kato and Launder modification (d-f) for L/D $=2$ at three stations: $\mathrm{x} / \mathrm{D}=0.5,1$ and 2.5 .

Figure 4.33. Calibrating normal-to-wall RMS fluctuation using SST model without Kato and Launder modification (a-c), and with Kato and Launder modification (d-f) for L/D $=6$ at three stations: $\mathrm{x} / \mathrm{D}=0.5,1.5$ and 3 .

Figure 4.34. Deposition density for $\mathrm{L} / \mathrm{D}=2$ from turbulent tracking and near-wall correction at different $\mathrm{y}+$ using modified EIM and: (a) SST without Kato and Launder modification, and (b) SST with Kato and Launder modification. 125

Figure 4.35. Deposition density for $\mathrm{L} / \mathrm{D}=4$ from turbulent tracking and near-wall correction at different $\mathrm{y}+$ using modified EIM and: (a) SST without Kato and Launder modification, and (b) SST with Kato and Launder modification. 127

Figure 4.36. Deposition density for $\mathrm{L} / \mathrm{D}=6$ from turbulent tracking and near-wall correction at different $\mathrm{y}+$ using modified EIM and: (a) SST without Kato and Launder modification, and (b) SST with Kato and Launder modification. 130

Figure 5.1. Mean velocity magnitude using RANS RSM-BSL model for L/D $=2$ at three stations: (a) $x / D=0.5$, (b) $x / D=1$, and (c) $x / D=2.5$. 137

Figure 5.2. RMS fluctuating components using RANS RSM-BSL model for L/D $=2$ at three stations: $\mathrm{x} / \mathrm{D}=0.5,1$ and 2.5 . 138

Figure 5.3. Mean velocity magnitude using RANS RSM-BSL model for L/D $=6$ at three stations: (a) $\mathrm{x} / \mathrm{D}=0.5$, (b) $\mathrm{x} / \mathrm{D}=1.5$, and (c) $\mathrm{x} / \mathrm{D}=3$. 141 
Figure 5.4. RMS fluctuating components using RANS RSM-BSL model for $\mathrm{L} / \mathrm{D}=6$ at three stations: $\mathrm{x} / \mathrm{D}=0.5,1.5$ and 3 . 142

Figure 5.5. Deposition density from turbulent tracking and mean flow tracking for $\mathrm{L} / \mathrm{D}=$ 2 using RSM-BSL/modified EIM. 145

Figure 5.6. Deposition density from turbulent tracking and mean flow tracking for $\mathrm{L} / \mathrm{D}=$ 4 using RSM-BSL/modified EIM. 145

Figure 5.7. Deposition density from turbulent tracking and mean flow tracking for $\mathrm{L} / \mathrm{D}=$ 6 using RSM-BSL/modified EIM. 146

Figure 5.8. Calibrating RMS normal-to-wall fluctuation using RSM-BSL model for L/D $=2(\mathrm{a}-\mathrm{c})$, and $\mathrm{L} / \mathrm{D}=6(\mathrm{~d}-\mathrm{f})$. 150

Figure 5.9. Deposition density for $\mathrm{L} / \mathrm{D}=2$ from turbulent tracking and near-wall correction with the new function at different $y+$ using RSM-BSL/modified EIM..... 152

Figure 5.10. Deposition density for $\mathrm{L} / \mathrm{D}=4$ from turbulent tracking and near-wall correction with the new function at different $y+$ using RSM-BSL/modified EIM..... 153

Figure 5.11. Deposition density for $\mathrm{L} / \mathrm{D}=6$ from turbulent tracking and near-wall correction with the new function at different $y+$ using RSM-BSL/modified EIM.... 154

Figure 5.12. Sensitivity of the Lagrangian time scale constant. ................................ 155

Figure 5.13. Effect of isotropic and anisotropic eddy lifetime scales on deposition..... 157

Figure 5.14. Particle deposition patterns for $\mathrm{L} / \mathrm{D}=2,4$ and 6 plotted for: $(\mathrm{a}, \mathrm{b}, \mathrm{c})$ turbulent tracking, and (d,e,f) near-wall correction. 159

Figure 5.15. Comparison of deposition density for L/D $=2$ using SST and RSM-BSL along with modified EIM. 161

Figure 5.16. Comparison of deposition density for $\mathrm{L} / \mathrm{D}=4$ using SST and RSM-BSL along with modified EIM. 161

Figure 5.17. Comparison of deposition density for L/D $=6$ using SST and RSM-BSL along with modified EIM. 162

Figure 5.18. Velocity magnitude contours ( $\mathrm{x}-\mathrm{z}$ plane, $\mathrm{y}=0$ ) for $\mathrm{L} / \mathrm{D}=2,4$ and 6 using SST and RSM-BSL model. Value is in $\mathrm{m} / \mathrm{s}$. 165

Figure 5.19. Kinetic energy contours $(\mathrm{x}-\mathrm{z}$ plane, $\mathrm{y}=0$ ) for $\mathrm{L} / \mathrm{D}=2,4$ and 6 using SST and RSM-BSL model. Value is in $\mathrm{m}^{2} / \mathrm{s}^{2}$. 166 
Figure 6.1. Schematic of the computational domain of the impinging jet based on the experiment of Burwash et al. (2006).....

Figure 6.2. Grid of the computational domain of the impinging jet used for LES calculations for: (a) Top view of the computational domain, and (b) Zoom of the normal-to-wall mesh in $\mathrm{x}-\mathrm{z}$ plane. 171

Figure 6.3. Effect of grid size on the particle deposition using LES. 174

Figure 6.4. Particle deposition density using LES compared with RANS calculations. 175

Figure 6.5. Particle deposition patterns for $\mathrm{L} / \mathrm{D}=2$ plotted for: (a) turbulent tracking using RANS/EIM, (b) near-wall correction using RANS/EIM, (c) using LES with dynamic perturbations, and (d) LES with constant perturbations.......................... 179

Figure C.1. Velocity magnitude along the centerline axis......................................... 204

Figure C.2. Velocity along a line parallel to the impingement wall........................... 205

Figure C.3. Kinetic energy profile along the centerline axis.................................... 206

Figure C.4. Kinetic energy profiles along a line parallel to the impingement wall....... 206 


\section{Nomenclature}

\begin{tabular}{|c|c|}
\hline$a$ & Spherical particle radius $[\mathrm{m}]$ \\
\hline$C$ & Lagrangian time scale constant [-] \\
\hline$C_{S}$ & Smagorinsky constant \\
\hline$C_{c}$ & Slip factor [-] \\
\hline$D$ & Nozzle diameter $[\mathrm{m}]$ \\
\hline$D_{\text {wall }}$ & Van Driest wall damping [-] \\
\hline$d$ or $d_{p}$ & Particle diameter $[\mu \mathrm{m}]$ \\
\hline $\mathrm{F}$ & Total force exerted on the particle $[\mathrm{N}]$ \\
\hline$F$ & $\begin{array}{l}\text { Function used in blending process in SST and RSM-BSL turbulence } \\
\text { models [-] }\end{array}$ \\
\hline$g$ & Gravitational acceleration $\left[\mathrm{m} / \mathrm{s}^{2}\right]$ \\
\hline$k$ or $\mathrm{k}$ & Turbulence kinetic energy $\left[\mathrm{m}^{2} / \mathrm{s}^{2}\right]$ \\
\hline$L$ & $\begin{array}{l}\text { Distance from the nozzle exit to the impingement wall }[\mathrm{m}] \text {, also used } \\
\text { as the side length of local element. }\end{array}$ \\
\hline Le & Eddy characteristic length scale $[\mathrm{m}]$ \\
\hline$L \min$ & Minimum side length of hexahedral element \\
\hline$m$ & Mass $[\mathrm{kg}]$ \\
\hline$M$ & Number of particles \\
\hline$N$ & Random number [-], also used as a number in Trilinear interpolation. \\
\hline$\varepsilon$ & Turbulence kinetic energy dissipation $\left[\mathrm{m}^{2} / \mathrm{s}^{3}\right]$ \\
\hline$\omega$ & Turbulence frequency $\left[\mathrm{s}^{-1}\right]$ \\
\hline$t$ & Time $[\mathrm{s}]$ \\
\hline$t_{0}$ & Starting time $[\mathrm{s}]$ \\
\hline$t_{i}$ & Initial time $[\mathrm{s}]$ \\
\hline $\operatorname{Re}$ & Fluid phase Reynolds number [-] \\
\hline $\mathrm{Re}_{\mathrm{p}}$ & Particle Reynolds number[-] \\
\hline Stk & Stokes number[-] \\
\hline$P$ & Air pressure $[\mathrm{Pa}]$ \\
\hline$T$ & Characteristic time scale [s] \\
\hline $\mathrm{y}$ & Distance to nearest wall [m] \\
\hline $\mathrm{y}^{+}$ & Nondimensional wall distance [-] \\
\hline$r$ & Radius [m] \\
\hline$u_{p 0}$ & Initial velocity of the particle $[\mathrm{m} / \mathrm{s}]$ \\
\hline$u_{e}$ & Eddy characteristic velocity scale $[\mathrm{m} / \mathrm{s}]$ \\
\hline$u^{*}$ & Friction velocity $[\mathrm{m} / \mathrm{s}]$ \\
\hline$V_{p}$ & Volume of single particle $\left[\mathrm{m}^{3}\right]$ \\
\hline$U$ & Velocity of the fluid $[\mathrm{m} / \mathrm{s}]$ \\
\hline
\end{tabular}




\begin{tabular}{|c|c|}
\hline$V$ & $\begin{array}{l}\text { Volume occupied by particles and fluid }\left[\mathrm{m}^{3}\right] \text {, also used as the particle } \\
\text { velocity }[\mathrm{m} / \mathrm{s}]\end{array}$ \\
\hline$\Phi_{p}$ & Volume fraction of particles $[-]$ \\
\hline$\ell$ & Length scale of small eddies in LES [m] \\
\hline$\ell$ & Length scale of energy containing eddies [m] \\
\hline$q$ & Velocity scale of small eddies in LES [m/s] \\
\hline$x, y, z$ & Cartesian coordinates \\
\hline$a, b, c$ & Local system coordinates in isoperimetric element \\
\hline$\rho$ & Density $\left[\mathrm{kg} / \mathrm{m}^{3}\right]$ \\
\hline$\mu$ & Molecular viscosity [Pa.s] \\
\hline$\mu_{t}$ & Turbulent viscosity [Pa.s] \\
\hline$v$ & Kinematic molecular viscosity $\left[\mathrm{m}^{2} / \mathrm{s}\right]$ \\
\hline$v_{T}$ & Kinematic eddy viscosity (also referred to as eddy viscosity) $\left[\mathrm{m}^{2} / \mathrm{s}\right]$ \\
\hline$\tau$ & Time $[\mathrm{s}]$ \\
\hline$\tau_{p}$ & Particle relaxation time or particle response time $[\mathrm{s}]$ \\
\hline $\bar{\tau} \tau_{e}$ & $\begin{array}{l}\text { Turnover time of large eddy, also used as eddy characteristic time } \\
\text { scale (or lifetime) [s] }\end{array}$ \\
\hline$\tau_{L}$ & Lagrangian time scale $[\mathrm{s}]$ \\
\hline$\tau_{K}$ & Kolmogorov time scale $[\mathrm{s}]$ \\
\hline$\Delta t$ & Time step [s] \\
\hline$\Delta$ & Filter width $[\mathrm{m}]$ \\
\hline$\varnothing$ & Nodal value \\
\hline$\varepsilon_{v}$ & Viscous dissipation used in LES $\left[\mathrm{m}^{2} / \mathrm{s}^{3}\right]$ \\
\hline$\tau_{w}$ & Wall shear stress $\left[\mathrm{kg} / \mathrm{m} . \mathrm{s}^{2}\right]$ \\
\hline$\Omega$ & Magnitude of vorticity rate $\left[\mathrm{s}^{-1}\right]$ \\
\hline$\delta_{i j}$ & Kronecker Delta [-] \\
\hline$U_{B}$ & Bulk velocity $[\mathrm{m} / \mathrm{s}]$ \\
\hline$U_{j}$ & Jet exit velocity $[\mathrm{m} / \mathrm{s}]$ \\
\hline$U_{\text {nozz }}$ & Fluid velocity upstream the nozzle exit $[\mathrm{m} / \mathrm{s}]$ \\
\hline$u$ & Velocity component in $\mathrm{x}$-direction $[\mathrm{m} / \mathrm{s}]$ \\
\hline$v$ & Velocity component in y-direction [m/s] \\
\hline$w$ & Velocity component in z-direction [m/s] \\
\hline$\gamma$ & User defined ratio in Equation $4.2[-]$ \\
\hline
\end{tabular}

Subscripts

\begin{tabular}{l|l}
$r m s$ or rms & Root-mean-square
\end{tabular} 


\begin{tabular}{l|l}
$f$ & fluid \\
\hline$p$ & particle \\
\hline$e$ & eddy \\
\hline$u, v, w$ & velocity components \\
\hline min & minimum \\
\hline Wall or w & wall surface \\
\hline $\mathrm{B}$ & bulk \\
\hline corr & correction \\
\hline $\mathrm{cr}$ & critical \\
\hline $\mathrm{j}$ & jet \\
\hline$K$ & Kolmogorov \\
\hline mag & magnitude
\end{tabular}

\section{Superscripts}

\begin{tabular}{l|l}
, & Prime (fluctuation) \\
\hline &
\end{tabular}

Abbreviations

\begin{tabular}{l|l} 
RANS & Reynolds averaged Navier Stokes \\
\hline LES & Large eddy simulation \\
\hline DNS & Direct numerical simulation \\
\hline CFD & Computational fluid mechanics \\
\hline SGS & Subgrid scales \\
\hline$C D k \omega$ & Cross-diffusion \\
\hline CFL & Courant-Friedrichs-Lewy number \\
\hline RMS & Root-mean-square \\
\hline EIM & Eddy interaction model \\
\hline SST & Shear stress transport \\
\hline RSM & Reynolds stress model \\
\hline &
\end{tabular}




\section{Chapter 1: Introduction}

\subsection{Impinging jet}

A turbulent jet impinging vertically onto a surface has great importance in many engineering and industrial applications. Due to its production of high levels of heat and mass transfer in the stagnation region, the impinging jet can be used, for example, in internal cooling of gas turbine blade, cooling of electronic devices and drying of paper or textile products. Another reason that makes the study of impinging jet an interesting subject is the flow complexity. The motion in the region close to the stagnation point involves a nearly irrotational normal straining. Also, the edge of the impinging jet has steep streamline curvature and strong rotationality, leading to a wall jet with hard-topredict features. Moreover, the impinging jet can easily be handled from a numerical point of view, and its numerical prediction provides a perfect test case for turbulence model assessment, as demonstrated by Craft et al. (1993), who examined four turbulence models (one k- $\varepsilon$ turbulence model and three second moment closures) using impinging jet as the test case. To understand the mechanism of the impinging jet flow, a brief description of the impinging jet flow characteristics is made. According to Jambunathan et al., (1992), the impinging jet has the following zones (see Figure 1.1):

(1) Initial mixing zone: The fluid from the surroundings is entrained into the jet. This shear or mixing region surrounds a core region, where the fluid velocity is nearly equal to the nozzle velocity. The core region is referred as the potential core region, even though the flow is not inviscid. The end of the core region is defined as the point where the axial velocity is 0.95 the nozzle exit velocity. 


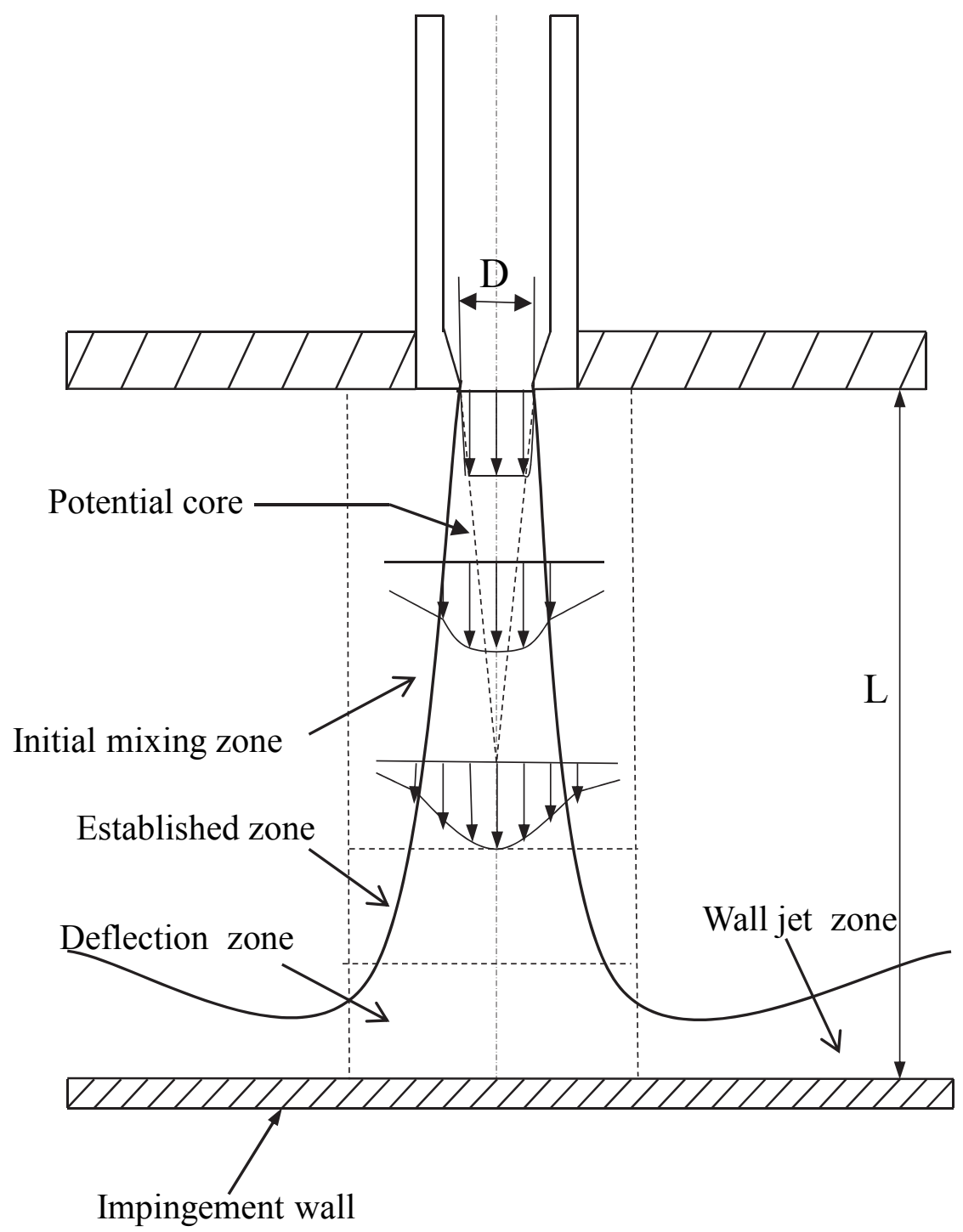

Figure 1.1. Impinging jet zones.

(2) Established zone: If the impingement wall is placed at a distance greater than the core length, the axial velocities will be reduced with increasing distance from the nozzle exit. The fall of the centerline velocity and the jet half width are directly proportional to the axial distance from the end of the potential core.

(3) Deflection zone: The region near the impingement plate is the deflection zone, where 
the axial velocity decreases rapidly and the static pressure is increased.

(4) Wall jet: Within the wall jet region, the velocity profile forms such that the velocity increases rapidly to a maximum near the wall and then falls for larger distances from the wall. The wall jet has higher levels of heat transfer than a similar parallel flow, which appears to be caused by the shear between the wall jet and the ambient air being transported to the boundary layer at the impingement wall.

The impinging jet has certain characteristics and features. Indeed, it has some differences compared to flows parallel to the walls (Cooper et al., 1993). These differences are highlighted in Table 1.1.

Table 1.1. Differences between the impinging jet and the flow parallel to walls (Cooper et al., 1993).

\begin{tabular}{|c|c|c|}
\hline Number & Impinging jet & Flow parallel to the wall \\
\hline 1) & $\begin{array}{c}\text { Very close to the axis of symmetry, } \\
\text { energy is produced by normal } \\
\text { straining. }\end{array}$ & Energy is produced due to shear. \\
\hline 2) & $\begin{array}{l}\text { The root-mean-square (RMS) } \\
\text { fluctuating velocity normal-to-wall } \\
\text { is higher than other components }\end{array}$ & $\begin{array}{l}\text { The RMS fluctuating velocity } \\
\text { normal-to-wall is smaller than other } \\
\text { components. }\end{array}$ \\
\hline 3) & $\begin{array}{l}\text { Turbulent length scales close to the } \\
\text { impingement wall are influenced } \\
\text { by the length scales of the jet } \\
\text { turbulence }\end{array}$ & $\begin{array}{l}\text { The length scales are usually } \\
\text { determined by the distance from the } \\
\text { wall. }\end{array}$ \\
\hline 4) & $\begin{array}{l}\text { Convective transport of turbulence } \\
\text { energy towards the stagnation point } \\
\text { is significant. }\end{array}$ & $\begin{array}{l}\text { Convective transport is usually } \\
\text { negligible. An approximate balance } \\
\text { between the generation and the } \\
\text { dissipation processes exist. }\end{array}$ \\
\hline
\end{tabular}


Adding solid or liquid particles as a second phase in the impinging jet flow creates more complexity due to particle-fluid interactions in different regions of the flow. For this reason, the impinging jet flow laden with particles is not well studied as relatively few studies have been conducted (Anderson and Longmire, 1995). As stated earlier, a turbulent jet impinging vertically onto a surface produces high levels of heat and mass transfer in the stagnation region. Further augmentation of heat and mass transfer in the stagnation region can be obtained by the introduction of solid or liquid particles into the impinging jet. Kurosaki et al. (1990), who studied the mechanisms of heat and mass transfer of an impinging air jet laden with solid particles experimentally, attributed the heat transfer augmentation relative to a single phase flow to several factors. These factors include the unsteady heat conduction between particles and the heated wall, the convective heat transfer between particles and surrounding primary flow, and heat transfer due to radiation between particles and the heated wall or among the particles.

Solid or liquid particle-laden impinging jets have various applications ranging from inkjet printing, sand blasting, surface erosion, cascade impactors, gas turbine industry and pharmaceutical aerosols. Some pharmaceutical aerosols devices such as dry powder inhalers (DPIs), which deliver a specific quantity of drug to the lungs, involve impinging jet flows as well as jet and swirling flows. Dry powder inhalers deagglomerate adhered particles using turbulent impinging jet flow in order to increase the efficiency of the drug delivery to the lung (Finlay, 2001). However, this leads to an increase in particle deposition on the impaction surface. Particle deposition on the impaction surface is an essential factor, affecting the efficiency of several mechanical applications. Studying the 
particle deposition in impinging jet flow numerically is the most important objective in the present research.

\subsection{Two-phase interactions and deposition mechanisms}

\subsubsection{Two-phase flow interactions}

The two-phase, or particle-laden, turbulent flow is a mixture in which two phases are present. One phase is fluid, either gas or liquid, and the second phase is composed of particles that can be in the form of solid particles, liquid droplets or gas bubbles (simply referred to as "particles"). The latter phase is called the dispersed phase (Elghobashi, 1994). The fluid phase can be in a laminar or a turbulent regime. However, this research focuses on the turbulent regime. The two-phases interact dynamically and are in motion because of the external forces acting on them. The fluid phase transports due to the effect of external force such as pressure and gravity forces. In the particulate phase, the dispersed particles move due to the forces generated by the fluid motion such as the drag force. On the other hand, the dispersed particles, as a reaction to the fluid forces, may exert forces upon the fluid phase (Mashayek and Pandya, 2003).

To better understand particle-laden turbulent flows, a brief description of fluidparticle interaction is made. According to Elghobashi (1994), particle-laden turbulent flows can be classified based on the type of interaction between the particles and the turbulent flow. Elghobashi presented a classification map of these interaction regimes as shown in Figure 1.2. In this figure, $\Phi_{p}=M V_{p} / V$ is volume fraction of particles, $M$ is the number of particles, $V_{p}$ is the volume of a single particle and $V$ is the volume 
occupied by particles and fluid. $\tau_{p}=\rho_{p} d^{2} / 18 \rho_{f} v$ is the particle response time (describes the response time of the particles to the changes in the fluid phase motion), $\tau_{K}$ $=(v / \varepsilon)^{1 / 2}$ is Kolmogorov time scale, and $\tau_{e}=\ell / u$ is the turnover time of large eddy. The vertical coordinate $\tau_{p} / \tau_{e}$ is the time scale ratio of the particle relaxation time to the large eddy life time. This time $\tau_{p} / \tau_{e}$ is related to $\tau_{p} / \tau_{K}$ through the turbulent Reynolds number $\operatorname{Re}=\ell u / v$.

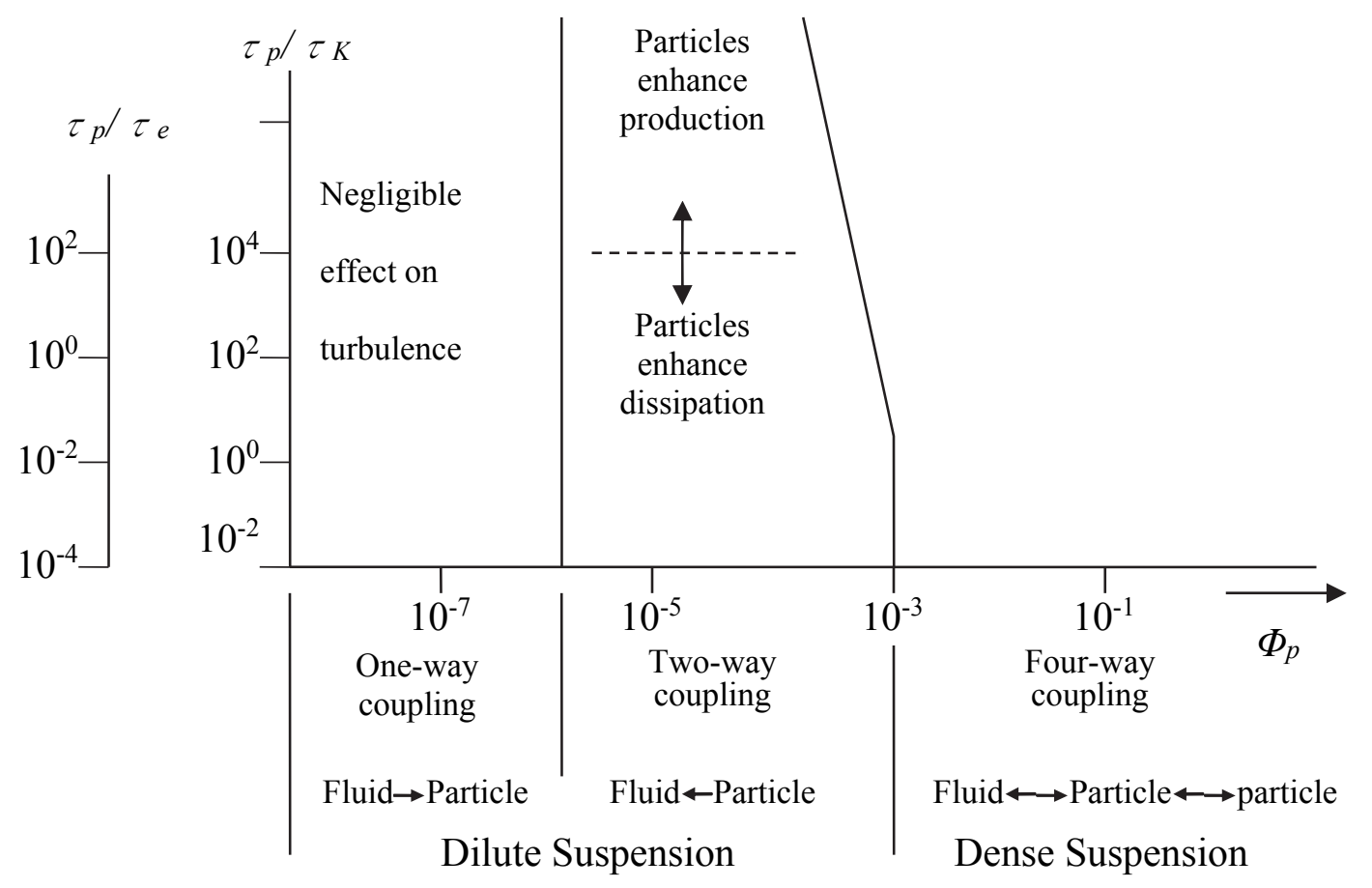

Figure 1.2. Map of regimes of fluid-particle interaction (adopted from Elghobashi, 1994).

In the above description, $\rho$ is the material density, and subscripts $p$ and $f$ denote the particle and fluid, respectively. $v$ is the kinematic viscosity of the fluid, $d$ is the diameter of the particle, $\ell$ is the length scale of the energy containing eddies, $u$ is the rms 
fluid velocity and $\varepsilon$ is the dissipation rate of turbulence kinetic energy. In the first regime, where the values of volume fraction of the particles are very low $\left(\Phi_{p} \leq 10^{-6}\right)$, the particles have negligible influence on the turbulent flow. The interaction between the turbulent fluid phase and the particulate dispersed phase in this case is known as one-way coupling. In this regime, the momentum transfer from the particles to the turbulent flow is insignificant because of the negligible concentration of the particles.

In the second regime, $10^{-6}<\Phi_{p} \leq 10^{-3}$, the momentum transfer from the particles to the turbulent flow is important and in this case can affect the turbulence structure. The interaction in this regime is called two-way coupling. In this regime, decreasing $\tau_{p}$ (particle response time) by decreasing the diameter of the particle for a given value of volume fraction of particles leads to an increase in the surface area of the particulate phase, and thus an increase in the dissipation rate of turbulence energy. However, as the particle response time increases for the same value of volume fraction of particles, the particle Reynolds number increases. When the particle Reynolds number reaches higher values $\left(\operatorname{Re}_{p} \geq 400\right)$, the vortex shedding occurs and the turbulence energy production is enhanced. In the third regime, $\Phi_{p}>10^{-3}$, in addition to the two-way coupling between the turbulent flow and the particles, the particle-particle interaction takes place and in this case the interaction is known as four-way coupling. Flows in this regime are referred to as dense suspensions due to the increased particle loading. 


\subsubsection{Deposition mechanisms}

The transport and deposition of particles on solid surfaces can take place in several physical processes such as the deposition of particles and droplets in gas, steam turbines and heat exchanger equipments. Similar physical processes occur in the atmospheric dispersal of pollutants, determination of indoor air quality, the transport and sedimentation of various substances in rivers, and deposition of drugs and harmful substances in the respiratory tract (medical engineering). In turbulent flows, the solid particles or liquid droplets, which are suspended in a fluid (gas or liquid), are transported and deposited on the solid wall due to certain mechanisms. These mechanisms include diffusion, gravity, inertial, thermophoresis and electrostatic effects (Williams and Hedley, 1973; Ganic and Mastanaiah, 1981; Sehmel, 1971).

Diffusion can be divided into two mechanisms: Brownian diffusion and turbulent diffusion. In Brownian diffusion, the particles collide randomly with surrounding air molecules, as they undergo a nondeterministic random walk motion (Finlay, 2001). This mechanism is significant for very small particles $d_{p}<<1 \mu m$, i.e., submicron, and nano particles. The turbulent diffusion, which is called turbulent dispersion mechanism (Burwash et al., 2006), is governed by the fluid turbulent fluctuations. In turbulent flows, the particles are transported by mean flow and dispersed near the wall by turbulent fluctuations that greatly enhance the possibility of the particles to hit the wall and hence cause the deposition. The turbulence dispersion mechanism is significant for aerosol particles with $d_{p}>1 \mu m$ and even for relatively larger ones (Abuzeid et al., 1991 and Lee and Gieseke, 1994). This means that this mechanism is important for most particle sizes and, moreover, becomes the dominant mechanism in the absence of other mechanisms. Li 
et al. (1994) concluded that since the particle deposition is affected by turbulent fluctuations, the turbulence dispersion mechanism is the dominating mechanism.

In gravitational mechanism, which is also called sedimentation, the particle in the fluid settles under the effect of gravity force and reaches a velocity that is called settling velocity (Finlay, 2001). Since gravity is the driving force of this mechanism, large particles are more influenced by this mechanism, especially when these particles reach the adjacent region of the wall. Abuzeid et al. (1991) pointed out that the gravitational mechanism is significant for particles greater than $30 \mu \mathrm{m}$ in diameter. This mechanism is more significant in horizontal flow systems than in vertical systems (McCoy and Hanratty, 1977).

The inertial mechanism includes the effects of impaction (also called impingement) and interception (Williams and Hedly, 1973). In inertial impaction, as the fluid velocity experiences a rapid change in magnitude and direction, a significant slip velocity arises especially for larger particles. When the fluid travels through a curved surface, the larger particles, due to their inertia, are unable to follow the rapid change in the fluid velocity and consequently collide with nearby solid surface and get deposited (Finlay, 2001). The inertial impaction mechanism is an important mechanism for highly curved surfaces (such as blades and gas turbines) or in complex flow systems where strong curvature behavior exists. The inertial interception takes place as the particle is shot by turbulence fluctuations and reaches a finite distance from the wall, equal to the particle radius, where protuberances of the wall surface enhance the deposition (Davies, 1965). The inertial interception is the dominant mechanism for the deposition on rough wall surfaces especially for large particles (Fan and Ahmadi, 1993). This mechanism depends on the 
particle to fluid density ratio and the wall roughness. This mechanism is important in the filtration processes for protection against dust of low toxicity as pointed by Lange et al. (2000).

Thermophoresis depends on the presence of a temperature gradient. When a particle is in a temperature gradient where some molecules are hot in one side and some are cold on the other side, the hot molecules, which have more energy than the cold ones, vibrate and thus cause large force. This force causes the particle to go in the direction of the cold molecules, towards the colder region (Greenfield and Quraini, 1998). This mechanism is significant for small particles.

Electrostatic effects are important for charged aerosol particles. The charged particles near the solid wall may experience an electrostatic force due to induced charges on the wall surface (mirror charging). This force due to mirror charging has an attractive nature and acts towards the wall, thus assisting deposition (Rao and Faghri, 1990).

\subsection{Research objectives}

This thesis presents a comprehensive research of aerosol particle dispersion and deposition prediction in jet flow impinging normally on an impingement wall. The impinging jet flow characteristics, which include recirculation and strong streamline curvature zones, as well as stagnation and free shear near-wall regions, are extremely complex. Consequently, the particle deposition on the impingement wall is also highly complicated. The influence of these characteristics on the deposition of particles is present in several applications. For example, in pharmaceutical aerosols, the deposition of particles in the mouth is caused by the impingement of the inlet jet on the back of the mouth, as pointed out in previous researches. Therefore, in relevance to that and aiming 
at better understanding of the physics of particle-turbulence interaction and deposition in impinging jet flow, a numerical prediction is necessary.

The single-phase impinging jet flow has been investigated both experimentally and numerically. In the literature, few studies have been reported for particle-laden jet impingement. These studies are mainly limited to experimental research. Very few numerical attempts have been made to study the particles' behaviors in impinging jet flows (see detailed discussion in Section 2.2). However, to the author's knowledge, none have conducted a numerical comprehensive investigation of aerosol particle dispersion and deposition for two-phase flow impinging normally over impingement wall (a flat plate). One part of the present research was to develop a new in-house tracking code for particle-laden impinging jet flows using modified EIM (eddy interaction model) as well as modified EIM in conjunction with near-wall correction technique. The modified EIM and near-wall correction technique to investigate the particle dispersion and deposition in impinging jet flows had not been accomplished prior to the present work.

Using the modified EIM in conjunction with the near-wall correction, two different approaches in the framework of RANS method were adopted in order to simulate aerosol particle dispersion and deposition in impinging jet flow: RANS SST, which is based on eddy viscosity assumption, and RANS RSM-BSL, which solves directly for the Reynolds stresses without modeling. Also, with one representative case of impinging jet flow, large eddy simulation with perturbed inflow condition was performed to predict the particle deposition in impinging jet flow. Results including particle deposition density and deposition patterns were predicted. Due to the novelty of these predictions, the results were validated with the experimental deposition measurements performed by Burwash et 
al. (2006). The results from the present work provide detailed information of aerosol particle dispersion and deposition process that can enhance and improve the understanding of the particle-turbulence interactions in such a complex flow.

Particular objectives of the present thesis were to:

1. Predict the aerosol particle deposition in the impinging jet flow.

2. Develop a new in-house tracking code that utilizes modified EIM; and adopt a methodology for exporting the flow field information and the connectivity information between the blocks and the elements that form the structured computational domains of three nozzle-to-surface distance $L / D$ cases of 2,4 and 6 , to improve the prediction of aerosol deposition in impinging jet flow. The height $L$ is the distance between the nozzle and the deposition surface and $D$ is the nozzle diameter.

3. Test the effectiveness of the classical EIM, which is implemented in the CFX tracking code, and apply eddy ratio correction to examine its limits in predicting the aerosol particle deposition.

4. Investigate the influence of the nozzle-to-surface distance on aerosol particle deposition using RANS method.

5. Adopt a near-wall correction technique based on impinging jet flow characteristics and implement this correction in the modified EIM.

6. Assess the performance of the modified EIM in conjunction with the near-wall correction, along with the two RANS approaches (SST and RSM-BSL models), to examine their limits in predicting aerosol particle deposition in impinging jet flow; and conduct large eddy simulation for $L / D=2$, using the perturbed inflow boundary condition and compare its prediction with RANS RSM-BSL/EIM to better understand the 
limits of applicability and accuracy of the different numerical methods on aerosol particle deposition.

\subsection{Thesis organization}

This thesis is composed of seven chapters. In Chapter 1, background, motivation and goals of the present research are presented. In Chapter 2, the literature relevant to the single-phase, the two-phase impinging jet, and the methods of prediction is reviewed. Chapter 3 introduces the numerical methods used in the current prediction, describes the in-house tracking code that was developed, and details the procedure of exporting the flow field data as well as the connectivity information. Chapter 4 presents the numerical results of RANS SST, along with the classical and modified EIM investigations. Chapter 5 presents the numerical results of RANS RSM-BSL, along with the modified EIM investigations. In Chapter 6, the particle deposition results using large eddy simulation for nozzle-to-surface distance of 2 are presented and compared with the numerical results of RANS RSM-BSL along with the modified EIM. Finally, the conclusions and future work are presented in Chapter 7. 


\section{Chapter 2: Literature survey}

More understanding of aerosol particle dispersion and deposition in impinging jet flow is important because the two-phase impinging jet flow case has received less attention than the single-phase case. Although several studies on particle-laden turbulent flows have been conducted, these studies are focused on pipe (or channel), free jet flow as well as some configurations related to pipe flows. In this chapter, relevant literature of single and two-phase impinging jet flows is reviewed. In addition, previous studies of different particle-laden turbulent flows using Reynolds averaged-Navier Stokes (RANS) and large eddy simulation (LES) methods are introduced.

\subsection{Single-phase impinging jet flow}

Single-phase impinging jet flow was the subject of several experimental and simulation studies. Some of these studies are summarized in Table 2.1. The impinging jet flow received more attention and focus on heat transfer aspects, due to its highly localized heating and cooling rates. There are many studies (e.g., Shi et al., 2002a; Shi et al., 2003a; Hattori and Nagano, 2004; Dano et al., 2005; Zhang et al., 2011) that focused on the heat transfer characteristics of impinging jet flow. Also, since the heat transfer rate is a function of several factors, several researchers studied the effect of these factors on heat transfer in several attempts. Some studies investigated the effect of nozzle geometry and nozzle-to-surface distance, L/D, (e.g., Obot et al., 1979a; Garimella and Nenaydykh, 1996; Colucci and Viskanta, 1996; Brignoni and Garimella, 2000), the confinement effect 
Table 2.1. Summary of previous studies on single-phase impinging jet flow.

\begin{tabular}{|c|c|c|c|c|c|}
\hline $\begin{array}{c}\text { Researcher } \\
\text { and year }\end{array}$ & $\begin{array}{l}\text { Study } \\
\text { type }\end{array}$ & Model & $\begin{array}{c}\text { Simulation } \\
\text { conditions at } \\
\text { inlet }\end{array}$ & Focus of study & Main results \\
\hline $\begin{array}{c}\text { Tailland \& } \\
\text { Mathien } 1967\end{array}$ & $\begin{array}{c}\text { Experime- } \\
\mathrm{nt}\end{array}$ & N/A & N/A & $\begin{array}{c}\text { Flow field } \\
\text { structure in wall } \\
\text { jet region }\end{array}$ & $\begin{array}{c}\text { Spreading rate of } \\
\text { the wall jet, mean } \\
\text { velocity }\end{array}$ \\
\hline Amano, 1983 & Simulation & $\begin{array}{c}\text { RANS } \\
\mathrm{k}-\varepsilon\end{array}$ & $\begin{array}{c}\text { Pipe nozzle; } \\
\text { Constant profile } \\
\text { for velocity, } \mathrm{k}, \varepsilon\end{array}$ & $\begin{array}{l}\text { Flow field and } \\
\text { effect of } L / D\end{array}$ & $\begin{array}{c}\text { Mean velocity } \\
\text { (parallel to } \\
\text { impingement wall), } \\
\text { skin friction } \\
\text { coefficient. } \\
\end{array}$ \\
\hline $\begin{array}{c}\text { Craft et al., } \\
1993\end{array}$ & Simulation & $\begin{array}{l}\text { RANS } \\
\mathrm{k}-\varepsilon \& \\
\mathrm{RSM}\end{array}$ & $\begin{array}{l}\text { Pipe nozzle; } \\
\text { fully developed } \\
\text { conditions }\end{array}$ & $\begin{array}{l}\text { Flow field under } \\
\text { effect of } L / D\end{array}$ & $\begin{array}{c}\text { Mean and RMS } \\
\text { fluctuating velocity } \\
\text { distribution }\end{array}$ \\
\hline $\begin{array}{l}\text { Nishino et al., } \\
1996\end{array}$ & $\begin{array}{c}\text { Experime- } \\
\mathrm{nt}\end{array}$ & N/A & $\mathrm{N} / \mathrm{A}$ & $\begin{array}{c}\text { Turbulence } \\
\text { statistics in } \\
\text { submerged water } \\
\text { jet }\end{array}$ & $\begin{array}{l}\text { Mean and RMS } \\
\text { fluctuating } \\
\text { velocities } \\
\text { distribution and } \\
\text { triple correlations } \\
\text { of velocity } \\
\text { fluctuations. }\end{array}$ \\
\hline $\begin{array}{c}\text { Knowles \& } \\
\text { Myszko, } 1998\end{array}$ & $\begin{array}{c}\text { Experime- } \\
\mathrm{nt}\end{array}$ & N/A & N/A & $\begin{array}{l}\text { Flow field and } \\
\text { turbulence in free } \\
\text { jet and } \\
\text { downstream wall } \\
\text { jet regions with } \\
\text { varying } L / D \\
\end{array}$ & $\begin{array}{c}\text { Mean velocities and } \\
\text { turbulent Reynolds } \\
\text { stresses }\end{array}$ \\
\hline $\begin{array}{c}\text { Rhea et al., } \\
2009\end{array}$ & Simulation & $\begin{array}{l}\text { RANS } \\
\text { RSM } \\
\& \text { LES }\end{array}$ & $\begin{array}{c}\text { Slot nozzle; } \\
\text { Fully developed } \\
\text { velocity profile } \\
\text { (RANS) and } \\
\text { velocity profile } \\
\text { obtained using } \\
\text { turbulence } \\
\text { generator (LES) }\end{array}$ & $\begin{array}{l}\text { Assessing ability } \\
\text { of RANS and } \\
\text { LES to predict } \\
\text { impinging jet }\end{array}$ & $\begin{array}{l}\text { Mean and RMS } \\
\text { fluctuating } \\
\text { velocities (parallel } \\
\text { to impingement } \\
\text { wall) }\end{array}$ \\
\hline $\begin{array}{l}\text { Seyedein et } \\
\text { al., } 1994\end{array}$ & Simulation & $\begin{array}{c}\text { RANS } \\
\mathrm{k}-\varepsilon\end{array}$ & $\begin{array}{l}\text { Pipe nozzle; } \\
\text { Constant } \\
\text { profiles for } \\
\text { velocity and } \\
\text { temperature }\end{array}$ & $\begin{array}{l}\text { Testing k- } \varepsilon \\
\text { performance by } \\
\text { predicting flow } \\
\text { field and heat } \\
\text { transfer }\end{array}$ & $\begin{array}{l}\text { Fluid flow includes } \\
\text { flow patterns \& } \\
\text { centerline velocity, } \\
\text { Nusselt number }\end{array}$ \\
\hline $\begin{array}{c}\text { Hattori \& } \\
\text { Nagano, } 2004\end{array}$ & Simulation & DNS & $\begin{array}{l}\text { Slot nozzle; } \\
\text { fully developed } \\
\text { conditions }\end{array}$ & $\begin{array}{c}\text { Structures \& } \\
\text { characteristics of } \\
\text { flow and heat } \\
\text { transfer }\end{array}$ & $\begin{array}{l}\text { RMS fluctuating } \\
\text { velocities, heat flux } \\
\text { and mean } \\
\text { temperature }\end{array}$ \\
\hline $\begin{array}{l}\text { Fuchs \& } \\
\text { Hallqvist, } \\
2009\end{array}$ & Simulation & LES & $\begin{array}{l}\text { Pipe nozzle; } \\
\text { sinusoidal } \\
\text { perturbation for } \\
\text { velocity profile }\end{array}$ & $\begin{array}{l}\text { Effects of inflow } \\
\text { conditions on } \\
\text { flow field and } \\
\text { heat transfer }\end{array}$ & $\begin{array}{c}\text { Mean \& RMS } \\
\text { fluctuating } \\
\text { velocities, Nusselt } \\
\text { number }\end{array}$ \\
\hline
\end{tabular}




\begin{tabular}{|c|c|c|c|c|c|}
\hline $\begin{array}{c}\text { Kubacki \& } \\
\text { Dick } \\
2010 \mathrm{~b}\end{array}$ & Simulation & $\begin{array}{l}\text { RANS } \\
\text { k- } \omega \text { \& } \\
\text { hybrid } \\
\text { RANS/L } \\
\text { ES }\end{array}$ & $\begin{array}{l}\text { Pipe nozzle; } \\
\text { Fully developed } \\
\text { conditions }\end{array}$ & $\begin{array}{c}\text { Evaluating } \\
\text { performance of } \\
\text { RANS and hybrid } \\
\text { RANS/LES }\end{array}$ & $\begin{array}{l}\text { Mean velocity, } \\
\text { RMS fluctuating } \\
\text { velocities and } \\
\text { Nusselt number }\end{array}$ \\
\hline $\begin{array}{l}\text { Garimella \& } \\
\text { Nenaydykh } \\
1996\end{array}$ & $\begin{array}{c}\text { Experime- } \\
\mathrm{nt}\end{array}$ & N/A & N/A & $\begin{array}{l}\text { Effect of nozzle } \\
\text { geometry (aspect } \\
\text { ratio) and } L / D \text { on } \\
\text { heat transfer. }\end{array}$ & $\begin{array}{l}\text { Distribution of } \\
\text { local heat transfer } \\
\text { coefficient }\end{array}$ \\
\hline $\begin{array}{l}\text { Shi et al., } \\
2003 \mathrm{a}\end{array}$ & Simulation & $\begin{array}{l}\text { RANS } \\
\mathrm{k}-\varepsilon \& \& \\
\text { RSM }\end{array}$ & $\begin{array}{c}\text { Pipe nozzle; } \\
\text { Constant } \\
\text { velocity profile }\end{array}$ & $\begin{array}{c}\text { Heat transfer } \\
\text { characteristics for } \\
\text { air jet impinging } \\
\text { normally and } \\
\text { obliquely into } \\
\text { imposed cross } \\
\text { flow } \\
\end{array}$ & $\begin{array}{c}\text { Nusselt number } \\
\text { distribution, } \\
\text { temperature } \\
\text { difference } \\
\text { between jet \& cross } \\
\text { flow }\end{array}$ \\
\hline $\begin{array}{l}\text { Wang \& } \\
\text { Mujumdar, } \\
2005\end{array}$ & Simulation & $\begin{array}{l}\text { RANS } \\
\mathrm{k}-\varepsilon\end{array}$ & $\begin{array}{l}\text { Slot nozzle; } \\
\text { constant profile } \\
\text { for velocity, } \\
\text { temperature, } \mathrm{k} \\
\& \varepsilon\end{array}$ & $\begin{array}{c}\text { Investigating } \\
\text { performance of } \\
\text { low Reynolds k- } \varepsilon \\
\text { in predicting flow } \\
\& \text { heat transfer } \\
\text { characteristics }\end{array}$ & $\begin{array}{l}\text { Nusselt number } \\
\text { distribution } \\
\text { reported only }\end{array}$ \\
\hline $\begin{array}{c}\text { Gao \& Ewing, } \\
2006\end{array}$ & $\begin{array}{c}\text { Experime- } \\
\mathrm{nt}\end{array}$ & N/A & N/A & $\begin{array}{c}\text { Confinement and } \\
L / D \text { effect on heat } \\
\text { transfer }\end{array}$ & $\begin{array}{l}\text { Nusselt number } \\
\text { distribution }\end{array}$ \\
\hline
\end{tabular}

(e.g., Seyedein et al., 1995; Gao and Ewing, 2006) and the effect of inflow conditions on flow field and heat transfer (e.g., Fuchs and Hallqvist, 2009). Table 2.1 describes some of these studies.

The single-phase impinging jet is a challenging test case for Computational Fluid Dynamics (CFD) because it exhibits extremely complex flow characteristics depite its relatively simple geometry (Park and Sung, 2001). As can be seen in Table 2.1, several numerical studies including RANS and LES approaches were conducted to test the performance of RANS turbulence models and assess the ability of LES to predict the flow and heat transfer characteristics (e.g., Rhea et al., 2009; Seyedein et al., 1994; Kubacki and Dick, 2010b; Wang and Mujumdar, 2005). 
There are several studies (e.g., Sigalla, 1958; Huang et al., 1984; Barata et al. 1993; Cooper et al., 1993; Craft et al., 1993; Nishino et al., 1996; Amano, 1983; Morries et al., 1999; Quispe et al, 2000) focused on investigating the flow field structure and turbulence statistics under the influence of different $L / D$ distance cases. Other studies (e.g., Tailland and Mathien, 1967; Knowles and Myszko, 1998) investigated the flow field with more attention on certain regions (free and wall jet regions) of the impinging jet flow.

Among the numerous flow field experiments is the experiment of Cooper et al. (1993), who performed an extensive set of measurements on the flow field. In their experiment, several $L / D$ distance cases ranging from 2 to 10 were tested. However, in their experiment they focused on the $L / D$ distances of 2 and 6 . In their results, they reported the mean velocity profiles in the vicinity of the impingement wall as well as the root-mean-square (RMS) fluctuating velocities. Their results for the mean velocity profiles showed small variations with $L / D$ distance. The RMS fluctuating velocities showed quantitative differences in the radial development according to $L / D$ distance cases although the qualitative behavior is similar in all the cases as shown in Figure 2.1. The authors noted a feature that is the level of the normal-to-wall RMS fluctuating velocity profile for $L / D=2$ is approximately half that of $L / D=6$. This can be clearly seen at $r / D=0.5$ (beginning of the wall jet) as demonstrated in Figure 2.1. They explained this difference by the fact that in lower nozzle-to-surface distances (i.e., $L / D<6$ ) the mixing layer does not spread to the jet centerline axis whereas in large nozzle-to-surface distances, where more mixing occurs before the jet strikes the impingement wall, the turbulence levels of RMS fluctuating velocities are higher. As the flow develops 
downstream the wall jet, the difference in RMS velocity between the two cases nearly vanishes.
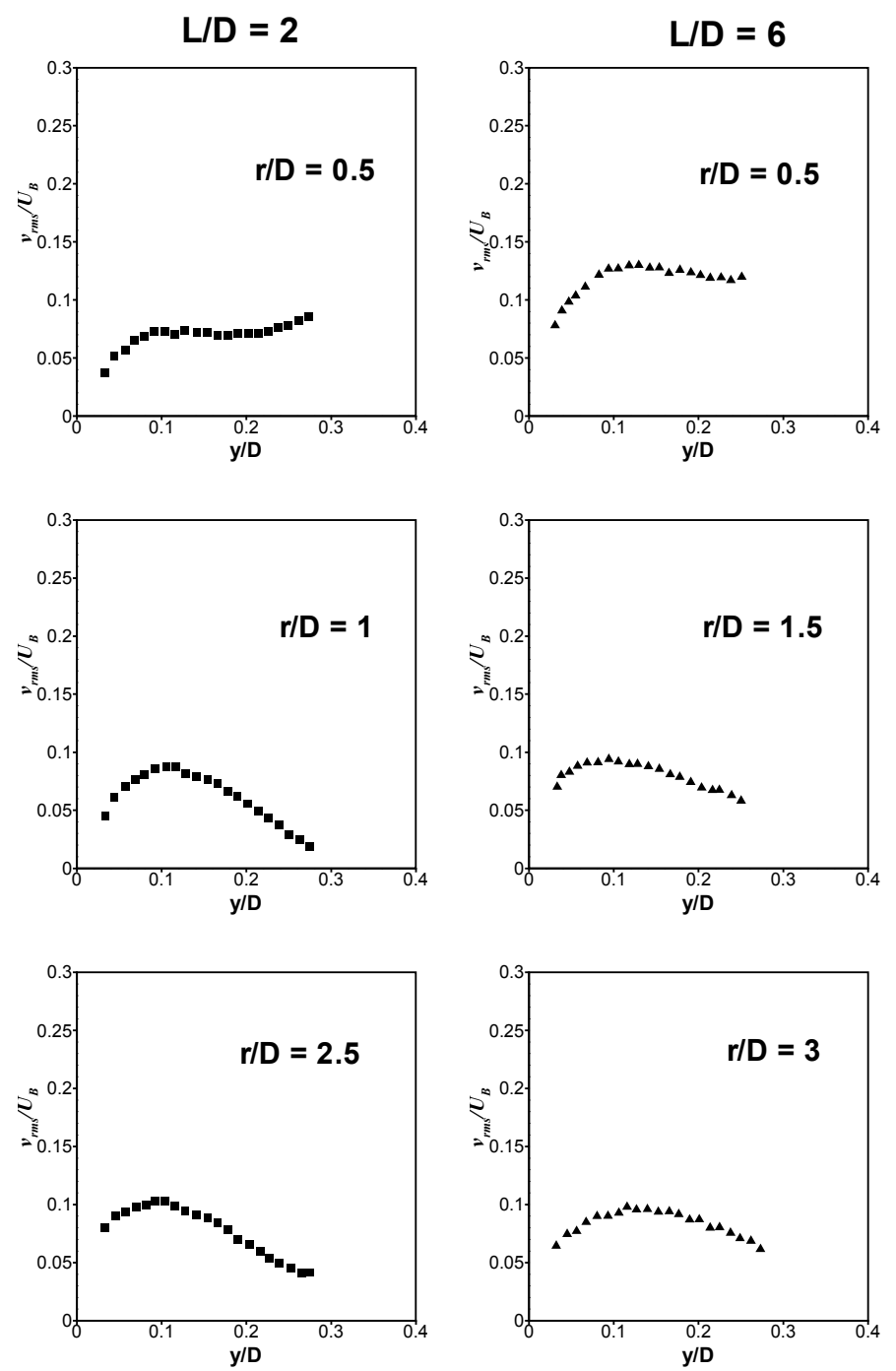

Figure 2.1. Normal-to-wall RMS fluctuatuating velocities for $L / D=2$ and 6. (Cooper et al., 1993)

Despite extensive research of both experimental and numerical single-phase impinging jet flow, the two-phase impinging jet is less investigated as will be highlighted in the next section. The particle-laden impinging jet flow, with the focus on dispersion and deposition process, is the subject of the present thesis. 


\subsection{Two-phase impinging jet flow}

As stated earlier, the single-phase impinging jet is a complex flow by its nature. Thus, adding solid, liquid or gas particles (particulate phase) increases this complexity due to the presence of interaction between the two phases. In comparison to the single-phase impinging jet, the two-phase impinging jet has not been investigated extensively.

The turbulent jet impinging vertically on a surface yields high levels of heat and mass transfer in the stagnation point, and adding solid or liquid particles causes further augmentation of heat and mass transfer. Shimizu et al., (1979) and Kurosaki et al. (1986) noticed this augmentation and indicated that there is a remarkable increase in the heat transfer rate around the stagnation point. Yoshida et al. (1990) examined the turbulence structure for the gas and solid phases and found that the turbulence intensity for the two phases is higher than that for the single phase, especially near the stagnation point, due to gas-solid interactions. As a result, this increase in turbulent intensity near the stagnation point leads to a marked heat transfer enhancement. There is a number of experimental and numerical studies that investigated the heat transfer produced by two-phase impinging jets (e.g.,Yoshida et al., 1988; Kurosaki et al., 1990; Yokomine et al., 2002; Shi et al., 2003b; Pakhomov and Terekhov, 2012).

The interaction of particles and the primary flow in the two-phase impinging jet as well as the influence of this interaction on the particle motion near the impingement wall were the subject of some studies. Anderson and Longmire (1995), in their experiment, found that the Stokes number, which can be defined as the ratio of particle to fluid time scales (Crowe et al., 1985), has a strong effect on particle behavior. For larger Stokes number $(S t k>0.6)$ the particles can not immediately adjust to the acceleration in the gas 
phase and hence deviate strongly from the fluid velocity. For smaller Stokes number, the particles respond more quickly to the fluid fluctuations. There are some numerical investigations (e.g., Dosanjh and Humphrey, 1985; Hatta et al., 1991) that showed similar findings as those of Anderson and Longmire (1995). However, these studies did not provide any quantitative analysis about particle deposition.

In a similar subject, Ciampini et al (2003a) developed a computer model for determination of the interference between an incident stream of spheres and those rebounding from a flat surface. Although their model is capable of examining several parameters on the inter-particle collisions, it suffers from several limitations. One of the important limitations is that the drag force and other fluid forces were neglected. Ignoring the effect of the fluid (air) forces on the particles allows the particle paths to be approximated by straight lines and makes the effect of air jet on the rebounding particles to be ignored. Ciampini et al (2003b) used the model developed by Ciampini et al (2003a) to study the following parameters on the inter-particle collisions: stream angle of incidence, nozzle divergence angle, particle velocity, particle size, particle flux, particleparticle and particle-surface impact parameters. Although these studies described the collision dynamics of the particles, they did not investigate the particle deposition in impinging jet.

Some experimental studies (Fuchs, 1964; Sethi and John, 1993; Marple, 2001; Gomez-Morino et al., 2002) investigated the particle deposition in configurations related to impinging jet flow, such as the cascade jet impactor, a device that is used for the collection of aerosol particles. In order to improve the design of jet impactors, Sethi and John (1993) investigated experimentally impaction patterns of particles over an impaction 
surface using an image analysis technique (John et al., 1991). The authors used $3 \mu \mathrm{m}$ ammonium fluorescent particles and observed that the deposition patterns formed a ring

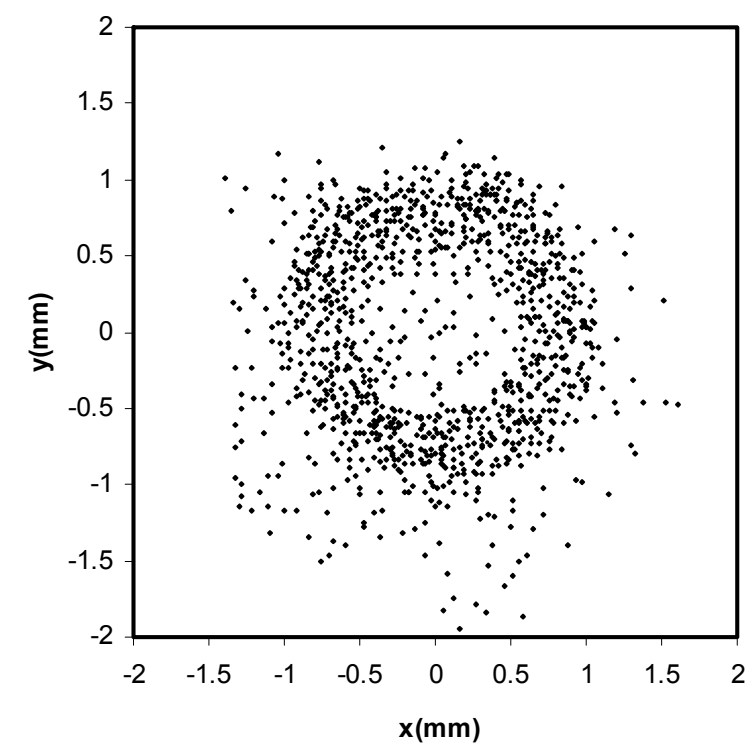

Figure 2.2. Impaction pattern. (adopted from Sethi and John, 1993)

shape on the impaction surface as shown in Figure 2.2. They also tested the effect of turbulence on the deposition pattern, by increasing the flow rate through the nozzle, and found that the annular deposition area of the ring is moving towards the center of the impaction surface. They therefore concluded that the turbulence effect is significant on the deposition patterns. Gomez-Morino et al. (2002), who studied experimentally the effect of turbulence transition in impactor jets, concluded that the turbulence has a significant effect on particle deposition.

There are numerical studies predicted the heat transfer influence on deposition efficiency in configurations involving internal impingement flow. Ai and Fletcher (2012) conducted numerical computations to simulate the gas and ash particles impinging on gas turbine disk at a $45^{\circ}$ angle, with film cooling fluid injection through a row of different- 
sized cylindrical holes distributed at varying hole spacing. In their simulation, they studied the effect of hole size and injection blowing ratio on capture efficiency, defined as the ratio of the mass of the adhered particles to the total mass of particles released in the domain. They found that the capture efficiency decays as the blowing ratio and hole size increase (as the blowing ratio is held fixed). Indeed, they studied the effect of adding thermal barrier coating (TBC), a layer with particular thermal conductivity placed over the flat disk, on the deposition over the surface. It was found that the TBC layer reduces the surface temperature initially. However, when a thin ash deposition layer forms, the thermal resistance layer increases the surface temperature, which in turn accelerate the deposition formation and thus decreases conductive heat transfer.

In an experimental study that focused on particle deposition in impinging jet flow, Burwash et al. (2006) conducted a set of measurements of the deposition of $5 \mu \mathrm{m}$ particles in impinging jet flow using three nozzle-to-surface distances, $L / D=2,4$ and 6 . In their experiment, the authors investigated the deposition density and the deposition patterns for the three cases of $L / D$. Their results showed that the deposition density for the impinging jet increased close to the stagnation point, when the $L / D$ distance was increased as can be shown in Figure 2.3. They attributed this behavior to the turbulent fluctuation velocities, which increase as the $L / D$ distance increases. They also found that the deposition peak over the impingement surface moves away from the stagnation point as the $L / D$ distance is decreased. This was attributed to the movement of the maximum turbulence kinetic energy's location as the $L / D$ distance is decreased. Their results for the particle deposition pattern indicate the ring shape pattern, which was previously observed by Sethi and John (1993). They concluded that the turbulent dispersion mechanism, due 
to turbulent diffusion by fluctuating velocities, is an important mechanism for particle deposition.

The literature review above revealed the fact that a comprehensive analysis of aerosol particle deposition through a numerical approach is lacking at the moment. The aerosol particle dispersion and deposition in impinging jet flow is investigated numerically in the present thesis.

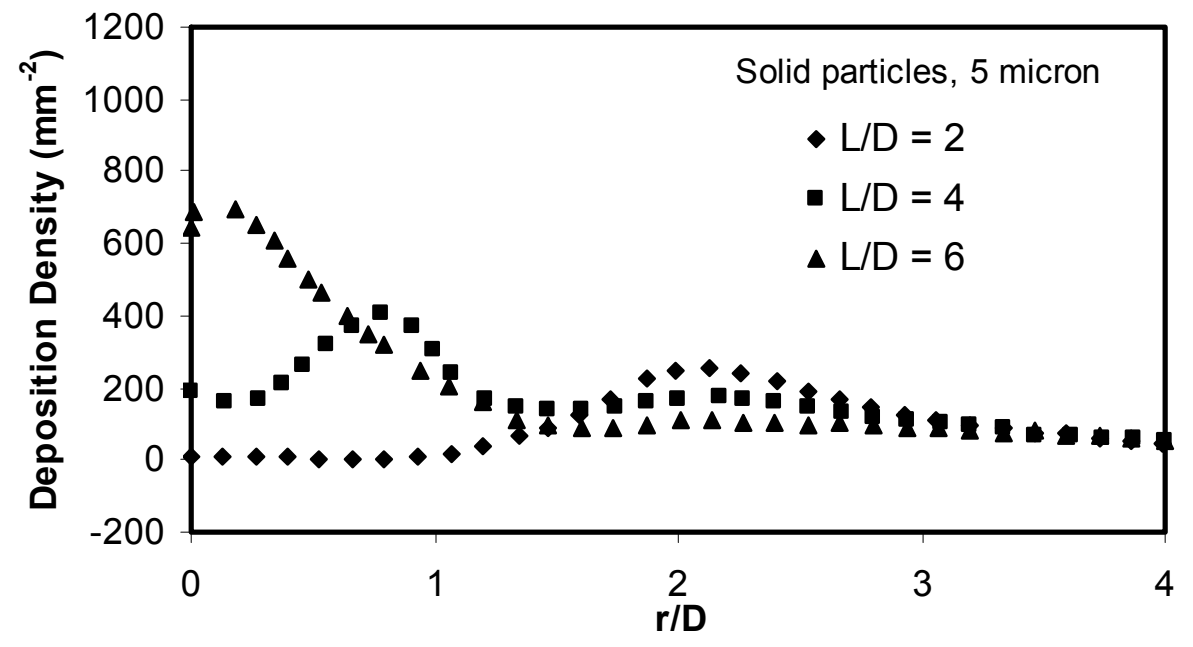

Figure 2.3. Deposition behaviour in impinging jet. (Burwash et al., 2006).

\subsection{Previous studies of different particle-laden flows}

\subsubsection{Using RANS approach}

The two-phase turbulent flows are commonly simulated using the Lagrangian or Eulerian approaches (Mashayek and Pandya, 2003). In the Eulerian approach, which is also called "two-continuum", the particulate phase can be treated as fluid in the Eulerian framework (Elghobashi, 1994). In the Lagrangian approach, a large number of particles is introduced into the flow and their trajectories are computed using the particle equation of motion. 
MacInnes and Bracco (1992) divided the Lagrangian approach into two types: the stochastic differential equation (SDE) and the random walk model. In the SDE models, an equation, which is called Langevin equation, is solved to obtain the fluid velocity fluctuation at the particle location. Several studies used different forms of the Langevin equation (see Thomson, 1987; He and Ahmadi, 1999 and Howorth and Pope, 1987). The random walk models, which are called also Monte Carlo models (Wang and Stock, 1992), do not need any equation to specify the Lagrangian velocity at the particle location. However, a random fluctuating velocity obtained from a Gaussian distribution, with zero mean and a variance related to the turbulent velocity scale that is computed from a turbulence model, is added to the local mean fluid velocity to determine the velocity at the particle location. There are several studies (e.g., Yuu et al., 1978; Dukowic, 1980; Ormancey and Martinon, 1984; Zhou and Leschziner, 1991) proposed various ways of simulating velocity fluctuation as either continuous turbulence or discontinuous turbulence. Although these Lagrangian approaches have a higher computational cost than the Eulerian approaches (Mashayek and Pandya, 2003 and Elghobashi, 1994), they can provide a more realistic and flexible models of particle deposition than the Eulerian approaches.

In the Lagrangian approach, the single-phase fluid flow is computed by RANS equations along with a turbulence model (for example, $k-\omega$, shear stress transport (SST), Reynolds stress model (RSM)), LES or DNS. Then, the particles (second phase) are introduced in the computational domain and their individual trajectories are computed using the equation of motion of particles. 
The most accurate approach for modeling particle deposition in turbulent boundary layer is DNS. The basic concept of DNS is to solve the Navier-Stokes equations on sufficiently fine grids without approximation. Although the use of DNS in several studies (e.g., McLaughlin, 1989 and Ounis et al., 1991) enhanced our understanding of the mechanisms controlling deposition, it is impractical due to its requirement for large computational overload. Therefore, RANS approach, which is more practical for industrial applications of flows with large Reynolds numbers, along with a turbulence model, is a cost effective way to compute the primary flow. It is also a preferred approach used to predict wall bounded flows such as impinging jet flow because of its simplicity and capability of predicting the near-wall flow by incorporating the damping functions. Moreover, it is well known that RANS approach with the turbulence model is able to predict the flow with reasonable accuracy (Wang and Mujumdar, 2005 and Jaramillo et al., 2008). The RANS equations can provide single-point turbulence statistical properties such as the mean and the variance of the fluid fluctuating velocity, which are obtained by turbulence closure models. Therefore, once the correct statistical properties are generated, the Lagrangian stochastic approaches in the framework of RANS are able to give an adequate prediction of particle deposition.

There have been several numerical studies that used the Lagrangian approaches in the framework of RANS approach. However, most of these studies were limited to simple flow systems such as pipe, channel, free jet flows and other relevant geometries. These studies are discussed and reviewed in the following subsections. 


\subsubsection{Particle-Laden Pipe Flow}

The particle-laden pipe (or channel) flows have been widely investigated in several numerical studies due to their numerous industrial applications. Li and Ahmadi (1992, 1993) studied numerically the dispersion and deposition of particles (in the range of 0.1 to $20 \mu \mathrm{m}$ in diameter) in a turbulent channel flow. In their simulation, they used previous mean velocity field data and the experimental data of Kreplin and Eckelmann (1979) for RMS fluctuating velocities. For the particulate phase, they followed Kraichnan's model (1970) to compute the local fluctuating velocities at the particle position. This model is based on generating a continuous Gaussian random field resembling a pseudo-isotropic turbulence in order to compute the instantaneous fluctuating velocity. In their simulation, the instantaneous fluctuating velocity was obtained by multiplying the experimental RMS fluctuating velocity by the product of local turbulence scale and the continuous Gaussian random number. The authors simulated the Brownian effect, which is significant for submicron particles (i.e., particles with a diameter $<1 \mu \mathrm{m})(\mathrm{Li}$ and Ahmadi, 1993), using Gaussian white noise random process.

Li et al. (1994) used the same approach as Li and Ahmadi, (1992, 1993), but the fluid phase was simulated by RANS along with algebraic stress turbulence model. Chen and Ahmadi (1997) also followed a similar approach; however, the authors used previous experimental data for the mean flow field and RMS fluctuation velocities. The deposition results of Li and Ahmadi, (1992, 1993), Li et al. (1994) and Chen and Ahmadi (1997) demonstrate a similar v-shape curve, as shown in Figure 2.4, due to the Brownian effect, which causes the deposition of submicron particles, and the gravitational effect, which causes the deposition of larger particles. Although the behavior of the v-shape curve is 
similar to previous simulations of Wood (1981) and MacLaughlin (1989), the results of Chen and Ahmadi (1997) were quite high for submicron particles when compared to Wood (1981). They attributed this to the fact that the surface curvature of the pipe wall increases the capture distance of the wall, and thus increases the deposition rate for those particles unlike the case for Wood's study, where the surface was flat.

Abuzeid et al. (1991), who simulated aerosol particles in turbulent channel flow, used a continuous random walk approach that is different from Kraichnan's model to

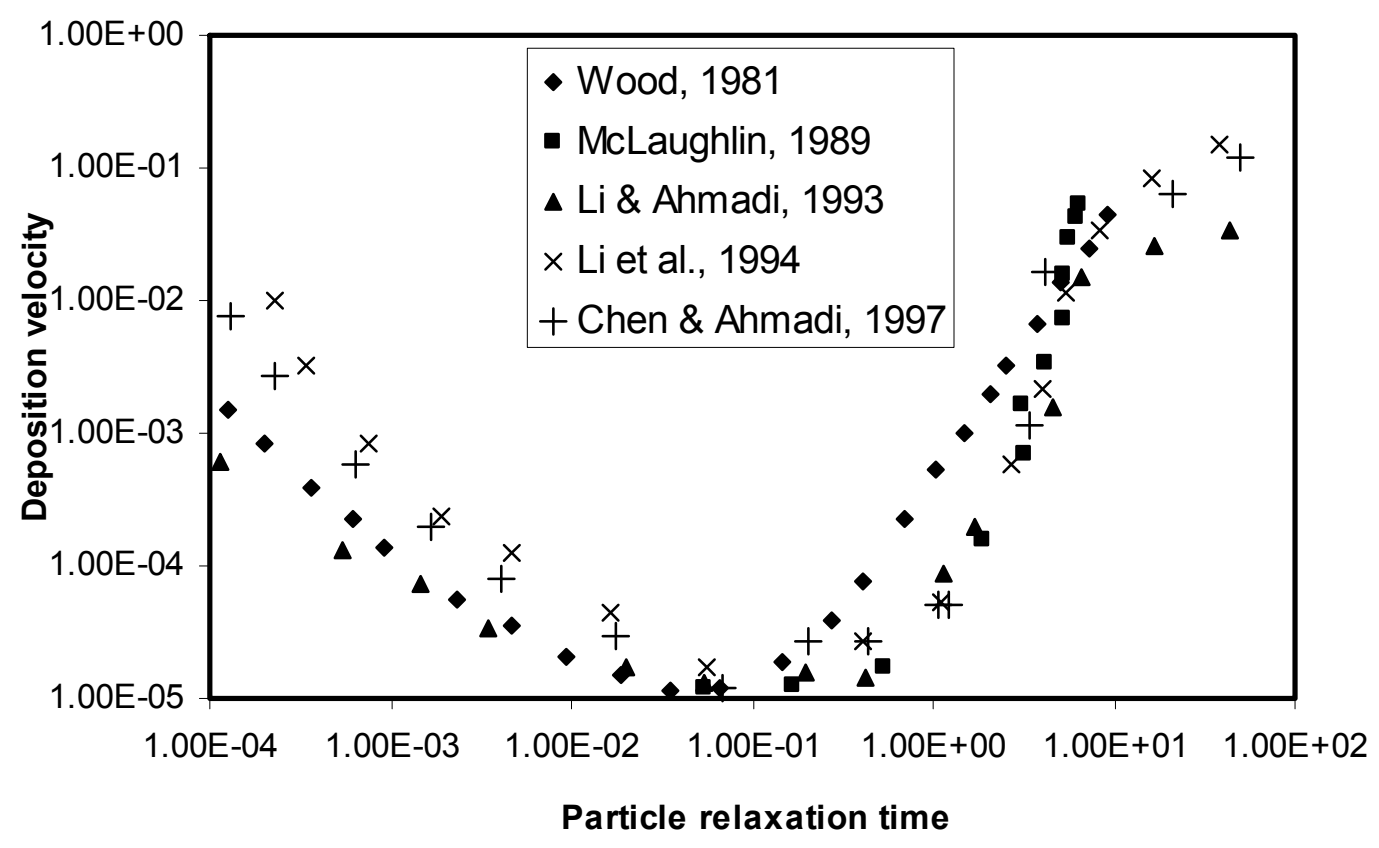

Figure 2.4. Particle deposition velocity versus nondimensional particle relaxation time. (adopted from Chen and Ahmadi, 1997).

simulate the particulate phase. In their approach, they assumed that the turbulence fluctuations are isotropic Gaussian process. Therefore, they used random time functions, which are a product of Gaussian random numbers, and variances of $2 k / 3, k$ here is the turbulence kinetic energy. These functions are generated at every time step, and used in 
an equation that introduces a nonzero correlation time to compute the fluctuating velocity at the particle position. The mean flow field and the turbulence kinetic energy were obtained from RANS along with k- $\varepsilon$ turbulence model. In their simulation, they found that the isotropy approximation causes the normal-to-wall fluctuating velocity, which is responsible for the particle deposition on the wall ( $\mathrm{Li}$ and Ahmadi, 1992), to be overestimated in regions close to the wall and this could affect the deposition. To remedy this problem, they used the normal-to-wall fluctuating velocities reported by Ahmadi and Chowdhury (1988) in the layer adjacent to the wall (inertial sublayer). Their results using these fluctuating velocities in the inertial sublayer showed better prediction than those using the isotropic assumption.

In order to have a better understanding of the physical details of the deposition mechanism within the turbulent boundary layer of a duct flow, Kallio and Reeks (1989) used a discontinuous random walk model to simulate the particulate phase in isotropic turbulence flow field. This model simulates the particle's trajectory as a succession of interactions with turbulent eddies, where each eddy is specified by lifetime, velocity, and sometimes length (Gosman and Ioannides, 1981, Graham and James, 1996). In their method, the authors obtained the eddy velocity by randomizing the RMS fluctuating velocity from a Gaussian probability distribution, and the eddy time scale by randomizing the Lagrangian integral time scale $\tau_{\mathrm{L}}$ from an exponential probability distribution. The RMS normal-to-wall fluctuating velocity was determined by incorporating its turbulence limit, which shows quadratic variation at short distance from the wall, into a curve fit of previous experimental data. The Lagrangian integral time scale was determined using an isotropic expression related to turbulence kinetic energy $k$, and dissipation $\varepsilon$, which are 
obtained from boundary layer measurements. The mean flow was established from previous experiments. The results for the particle deposition were in close agreement with previous experimental data. Sommerfield et al. (1993) followed Kallio and Reeks's method (1989) to study the dispersion in pipe flow, although they did not study the deposition.

Greenfield and Quraini (1997) also used the method of Kallio and Reeks (1989), but they obtained the RMS velocity and the eddy time scale based on an expression related to the kinetic energy and the dissipation from the fluid phase simulation. At large particle relaxation times, their results for particle deposition showed relatively close prediction to the experimental data; however, at small particle relaxation times, the deposition was slightly overpredicted. They attributed this overprediction to the small number of particles that were tracked in their simulation.

Similarly, Wang and James (1999b) used the discontinuous random walk to simulate the particulate phase, where the RMS velocity and the eddy time scale were obtained from the fluid phase, which is calculated by RANS along with the k- $\varepsilon$ turbulence model. The authors noted that the use of the RMS velocities in terms of the kinetic energy $k$, as obtained from the $\mathrm{k}-\varepsilon$ model, causes a significant difference between the simulation deposition results and the experimental data. To overcome this deficiency, they followed the method of Kallio and Reeks (1989), but by using DNS data of Kim et al (1987), which is curvefitted to obtain the variation of turbulent RMS velocities to account for the anisotropic effect in the buffer region close to the wall. Also, to circumvent this problem, Agnihotry et al. (2012) introduced the helicity effect, which is based on expressions related to the vorticity and mean velocity components, to account 
for the anisotropic behavior of the RMS velocities close to the wall of $90^{\circ}$ bend pipe. The results of Wang and James (1999b) and Agnihotry et al. (2012) were close to previous experiments.

Several numerical studies have used the discontinuous random walk model to simulate the particulate phase in pipe, duct flows or other related configurations. Example of these studies include Christoforou et al. (1992), Matida et al. (2000), Chorel et al. (2010), and Zhang et al. (2004).

In the literature, there are also many numerical studies that simulated the particulate phase using SDE based on different forms of the Langevin equation in framework of RANS. Among those studies is the study of Tian and Ahmadi (2007), who used RANS with k- $\varepsilon$ and RSM turbulence models to simulate the fluid phase, and SDE based on the Langevin equation to simulate the particulate phase. In their method, the instantaneous fluid velocity in the particle equation of motion is simulated for the given turbulence local mean velocity and fluctuating velocity using the Langevin equation (He and Ahmadi, 1999). The Lagrangian time scale, which is used in the Langevin equation, was computed from expressions related to the normal-to-wall fluctuating velocity and the turbulence dissipation when the RSM model was used, or from those that related to the turbulence kinetic energy $k$ and the dissipation $\varepsilon$ when the k- $\varepsilon$ model was used. In their simulation, they estimated the turbulence features close to the wall of the duct using standard wall function and two-layer zonal model (Chen and Patel, 1988).

Tian and Ahmadi (2007) found that the deposition results demonstrate significant difference in comparison with the experimental data. They attributed this difference to the limitations of the standard wall function and the two-layer zonal model, as well as 
their inability to correctly account for the turbulence features in the vicinity of the wall, especially for the turbulent fluctuating velocity normal to the wall. To improve the deposition prediction, the authors incorporated the limit of turbulent fluctuation as was pointed out by Kallio and Reeks (1989), into the turbulence fluctuation term in the Langevin equation. They found that using the normal-to-wall turbulence fluctuation limit improved the particle deposition results when the RSM model was used for the fluid phase simulation, more than when $\mathrm{k}-\varepsilon$ was used.

Recently, in extrathoracic airways (ETA) geometry, which is related to pipe and duct flows, Dehbi (2011) used SDE with a different form of the Langevin equation to simulate the particulate phase, and RANS along with the RSM model, to simulate the fluid phase. The author used this form of the Langevin equation to compute the turbulent fluid fluctuation for a given RMS fluctuating velocity at the particle position during the trajectory process. Then, the turbulent fluid fluctuation is added to the mean velocity to obtain the instantaneous fluid velocity at the particle position. In their simulation, they tested flow rates including $30 \mathrm{~L} / \mathrm{min}$ and $90 \mathrm{~L} / \mathrm{min}$. Their deposition results showed overestimation and underestimation in specific regions of the ETA geometry when compared to experimental data. The author attributed these failures to the inadequate modeling of the turbulent fluctuation.

There are several studies (e.g., Wang and James, 1998 and Zhao et al., 2007) that simulated the particle deposition using Lagrangian form in framework of RANS in waveplate mist eliminators, which are simple devices used to remove liquid droplets from gas flow geometry. However, these studies did not use any stochastic Lagrangian approach (continuous, discontinuous random walk or SDE) to simulate the particulate phase 
(droplets) and subsequently account for the turbulence effects on the droplets through their equation of motion. Their results for deposition demonstrated a large discrepancy with the experimental results; this discrepancy was attributed by the authors to the lack of the turbulent fluid fluctuation effect. James et al. (2003), Galletti et al. (2008) and Zamora and Kaiser (2011) used a discontinuous random walk model to simulate the particulate phase and thus accounted for the turbulence effects on the droplets in a similar way as the studies introduced earlier in this section. Although James et al. (2003) did not compare their results with experimental data, the results of Galletti et al. (2008), and Zamora and Kaiser (2011) showed an improved prediction and had better agreement with the experimental data.

\subsubsection{Particle-Laden Free Jet Flow}

Two-phase turbulent free jets were also investigated numerically using Lagrangian stochastic methods in framework of RANS in several studies due to their occurrence in many engineering applications. Yuu et al. (1978), in order to study the dispersion of particles in turbulent jet flow, used a discontinuous random walk model for simulating the particulate phase and empirical formulae to obtain time averaged fluid velocities. The RMS fluctuating velocity distributions were obtained from previous experimental data. In their model, they assumed that the particle is surrounded by an eddy, which has an eddy lifetime and a velocity equal to the RMS fluid fluctuation. The authors obtained the fluid fluctuating velocity by multiplying the RMS fluctuating velocity by a random number and chose the eddy lifetime to be equal to the Lagrangian time scale obtained from the Lagrangian velocity correlation of the fluid. 
In another form of the discontinuous random walk model, Shuen et al. (1983a) adopted a model where the eddy velocity, lifetime and size were obtained from the k- $\varepsilon$ turbulence model. The authors obtained the fluid fluctuating velocity by multiplying the RMS fluctuating velocity, which is quantified by $(2 k / 3)^{0.5}$, by a random number. In their model, they assumed the particle to stay within the eddy for a particular time, which is the minimum of eddy lifetime or transit time. The transit time was determined from eddy size and lifetime scales. Shuen et al. (1983b) extended the model used by Shuen et al (1983a) by allowing the particles to stay within the eddy as long as their residence times and displacements are less than the eddy lifetime and the eddy size. The authors used various particle sizes. Their results for the particle dispersion using the original and extended models were relatively close to experiments.

Mostafa and Mongia (1988) and Nijdam et al. (2006) used the model of Shuen et al. (1983a) to simulate the particle dispersion in free jet where the fluid phase was simulated by RANS along with the k- $\varepsilon$ turbulence model. However, they determined the eddy lifetime such that it depends inversely on the magnitude of the current fluctuation velocity.

Chen et al., (2001) used the model of Shuen et al. (1983a) with some modifications to simulate the dispersion of larger particles (15 to $115 \mu \mathrm{m}$ ), whereas the fluid flow was simulated by RANS along with the Reynolds stress model (RSM). Based on previous studies (e.g., Graham and James, 1996), the authors modified the way of determining the interaction time of heavy particles (i.e., particles with large diameters) by introducing a new time, tint, that allows those particles to interact with the eddies for a longer time. Therefore, the interaction time becomes either the minimum of $\mathrm{T}_{\max }$ and transit time, or 
twice the eddy lifetime for low relative velocity between the two phases, where $\mathrm{T}_{\max }=$ $3.16 \tau_{e}$. In the model of Shuen et al. (1983a), the particles were allowed to interact for a time less than the eddy lifetime and this consequently caused an underprediction of the dispersion of heavy particles (Chen et al., 2001 and Graham and James, 1996). By using the new time as suggested by the authors, heavy particles can disperse more in the long time limit (Chen et al., 2001).

There have been several numerical studies that investigated, in framework of RANS, the dispersion of particles in jet and shear layer flows, using continuous or discontinuous random walk models that require specific forms of Lagrangian or Eulerian velocity auto-correlation functions. These correlation functions are required to compute the turbulent fluctuation velocities that are added to the mean velocities, which are obtained from the mean flow calculations (RANS with turbulence model) at the particle position. However, these methods need more analysis and involve time-consuming computations (Wang and James, 1999a). Examples of the studies include Berlemont et al. (1990), Burry and Bergeles (1993), Zhou and Leschziner (1991, 1996), Yokomine et al. (1991) and Ormancey and Martinon (1992).

\subsubsection{Using LES approach}

In particle-laden turbulent flows, interactions between the particles and the primary fluid phase are a problematic research topic of both fundamental importance and practical interest. Additionally, the interaction is a challenging problem in complex wall-bounded flows, such as impinging jet flow, because it contains free shear as well as zones of strong streamline curvature. In recent years, DNS and LES have become powerful tools 
for the study of dispersion and deposition of particles in turbulent fluid flows. However, although DNS is impractical due to its demand for a large computational power, it has been used in several studies to investigate the two-phase flows. DNS was used to study the deposition in turbulent channel flows (e.g., McLaughlin, 1989; Ounis et al., 1991; Chen and McLaughlin, 1995) and the dispersion of particles in mixing layer and jet flows (e.g., Ling et al., 2000; Li et al., 2011).

The concept of LES, which is not as severely restricted in the range of Reynolds number and computational power as DNS, has emerged as a promising tool to address these types of problems. Moreover, its use has increased in computational turbulent flows. In LES, the large energy carrying eddies are computed directly whereas the influence of the small eddies (subgrid scales of motion) has to be modeled by a subgrid scale (SGS) model.

The use of RANS-based prediction methods rely significantly on turbulence models to represent all the relevant turbulent scales. Although RANS-based prediction methods can predict the particle deposition accurately to some extent, their predictions remain limited. Therefore, the recent improvements in the processing speed of computers make the applicability of LES to turbulent flows more feasible since it has less computational cost than DNS. Numerical methods based on LES can provide the optimum means of capturing the unsteady physical features of particle-laden turbulent flows. In LES, the particle dispersion is dominated mainly by the large-scale structures (Fan et al., 2001), which are obtained directly without modeling.

The particle dispersion and deposition in pipe (or channel) and shear turbulent flows have been the subject of many numerical studies. Several studies (e.g., Yeh and 
Lei, 1991; Uijttewall and Oliemans, 1996; Wang and Squires, 1996; Breuer et al., 2006; Berrouk et al., 2007; Berrouk and Laurence, 2008; Matida et al., 2006; Lei et al., 2008; Shotorban et al., 2007) have utilized LES to gain a better physical understanding of the particle-turbulence interactions.

One of the early studies, which investigated the motion of small particles in decaying isotropic turbulence flow generated by LES, is by Yeh and Lei (1991). In their research, they performed several simulations where the large-scale velocity (resolved velocity) was used in the particle equation of motion. The authors compared their results with previous experiments and concluded that the particle motion was mainly controlled by the motion of large-scales. Wang and Squires (1996) performed LES for the Reynolds numbers of 11,160 and 79,400 to investigate the particle deposition in a vertical turbulent channel flow. In their study, they used the large-scale velocity in the equation of motion of particles and found that the particle deposition rate is in agreement with the DNS results of McLauglin (1989) and previous experiments. They also tested the effect of SGS on the deposition by including SGS velocity fluctuations in the equation of motion of particles and found that their effects are insignificant.

The effect of SGS on deposition depends on the Reynolds number. For a fixed computational domain, increasing the Reynolds number corresponds to an increase in the turbulence energy in the SGS and hence a greater effect of small scale fluctuations on particle motion (Wang and Squires, 1996). Therefore, the effect of SGS turbulence will be more pronounced at higher Reynolds number (Berrouk et al., 2007).

Aiming at better understanding of particle deposition, Breuer et al. (2006) investigated the deposition of particles in $90^{\circ}$ bending pipe using LES for Reynolds 
number of 10,000 . In their research, they used the large-scale velocity in the equation of motion of particles. To study the effect of the Stokes number on the deposition of particles, they used various particle sizes, which correspond to the Stokes numbers of 0.001 to 1.5 . Their results for the deposition were in good agreement with previous experimental data over the entire Stokes number range considered as shown in Figure 2.5. From their findings, the authors noted that for large Stokes numbers the deposition

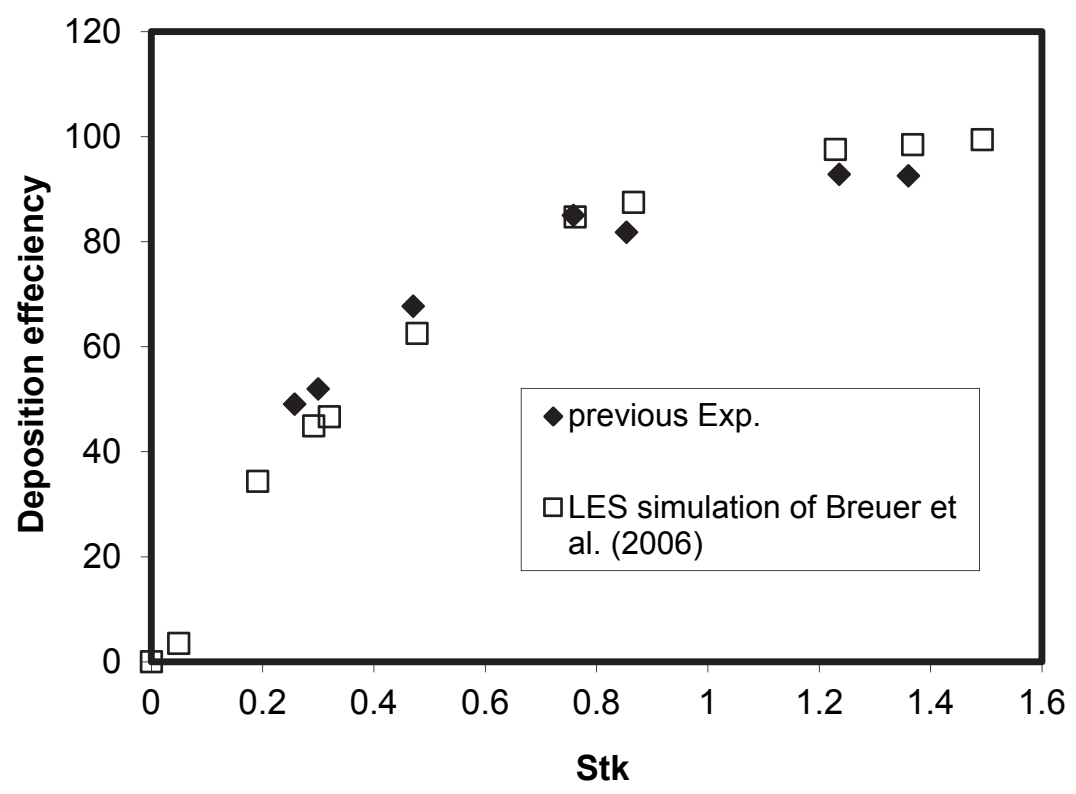

Figure 2.5. Deposition efficiency in turbulent bend flow (adopted from Breuer et al., 2006).

efficiency reaches $100 \%$, while for small Stokes numbers the efficiency goes to zero (see Figure 2.5). The authors attributed this behavior to the inertia of the particles. For small particles (small Stokes number), the inertia is low such that they are able to follow the primary flow closely and therefore leave the bend through the exit, except for a few particles that deposit at the side wall and at the inner bend. In contrast, for large particles, 
the inertia is high due to increasing of the particle sizes and consequently more particles are unable to follow the flow in the bend. As a result, more particles deposit in the bend.

In a similar study, Berrouk and Laurence (2008) performed LES to investigate the particle deposition in $90^{\circ}$ bend with and without including the SGS effect on the particles. Although they used a coarser mesh than Breuer et al. (2006), their results were close to the results of Breuer et al. (2006) and to previous experiments over the entire Stokes number range.

The behavior of particles in the primary fluid flow is strongly related to the largescale structures of the fluid. Thus, the particle dispersion is dominated by those large structures (Jin et al., 2003). There have been a number of numerical studies that investigated the particle dispersion in free jet flow using LES. Jin et al. (2003) performed LES to study the particle behavior and dispersion in free jet flow at Reynolds number of 11,300. They used particles with different diameters including 1, 10, 30 and $100 \mu \mathrm{m}$. In their LES, the authors used the large-scale velocity in the equation of motion of particles. By investigating the spatial distribution of the particles, the authors found that most of the particles follow the large-scale structures closely and cluster densely on the edges of these structures. However, the large particles $(100 \mu \mathrm{m})$ were less representative to this behavior. Several numerical studies were performed to investigate the particle dispersion in free jet flow. These studies include Fan et al. (2001), Yuu et al. (2001), Apte et al. (2003) and Almeida and Jaberi (2008). 


\subsection{Summary}

From the above literature, it can be deduced that there are several numerical studies focused on the deposition. However, these studies were limited to pipe and channel flow systems. Indeed, the above literature indicates that despite the two-phase impinging jet flow is investigated numerically, the deposition in the impinging jet is less explored. The present research proposes a comprehensive analysis regarding the prediction of aerosol particle dispersion and deposition in two-phase flow impinging normally over the impingement wall. This research has great significance in terms of improving the understanding of the fluid-particle interactions and the particle deposition in the impinging jet flow (Alatawi and Matida, 2012). The main findings in this research are to report the deposition density and the deposition patterns over the impingement wall for different nozzle-to-surface distances using several numerical approaches.

To predict the particle dispersion and deposition in the present research, two different approaches in framework of RANS method, namely RANS SST and RANS RSM-BSL, along with EIM were adopted to simulate aerosol particle dispersion and deposition in impinging jet flow. The classical EIM will be implemented along with the eddy ratio correction to test its effectiveness in predicting the aerosol particle deposition. In addition, the modified EIM, which is adopted in the in-house tracking code, and the modified EIM in conjunction with near-wall correction technique based on impinging jet flow characteristics to improve the particle deposition prediction will be implemented. Finally, LES prediction for $L / D=2$ using perturbed inflow boundary condition will be applied and its performance for the deposition prediction will be compared against RANS RSM-BSL/EIM. 


\section{Chapter 3: Numerical Methods}

The numerical simulation of impinging jet flow is a classical problem in turbulence. Moreover, adding a second phase (particulate phase) leads to significant challenges due to the strong influence of the fluid phase on the particulate phase, where the turbulent dispersion (turbulence fluctuations) is the main mechanism for small particles deposition.

It is critical to employ an appropriate model for simulating turbulence fluctuations for accurate analysis of aerosol particle transport and deposition processes. The most faithful simulation that is able to capture the details of the turbulence eddy structures is DNS. However, due to a wide range of length and time scales present in turbulent flows, DNS is still restricted due to large computational power required in relatively simple geometries. In DNS, the grid density required is proportional to $\operatorname{Re}^{3}$ (Luo et al., 2004), which requires tremendous resolution requirements.

The RANS approach along with a suitable turbulence model is a cost effective method to compute the primary flow. This makes it practical for different industrial applications and useful tool in industrial design. The RANS approach performs reasonably well in most of the impinging jet flow domain despite its complexity. However, the near-wall regions, including the stagnation and wall jet regions, are not properly resolved by most of the standard turbulence models (e.g., k- $\varepsilon$ and k- $\omega$ ). The modeling of these regions is significant issue in order to accurately predict the deposition results, especially for small particles, where the turbulent fluctuations drive the turbulent dispersion as the main mechanism for the particle deposition. Most of the solvers (like CFX Ansys Inc.) available nowadays employ turbulence models that are still formulated 
primarily, despite their sophistication, for turbulent flows in the core region. Therefore, to predict the particle transport and deposition accurately, it is important to model the nearwall region adequately. One of the advantages of the RANS approach is its capability and simplicity of predicting the near-wall flow by incorporating the damping functions. The present study introduces near-wall correction technique through the modified EIM to model the near-wall region in impinging jet flow domain. To accomplish this, an inhouse tracking code was developed in the present study. The shear stress transport (SST) turbulence model is used along with EIM to predict the particle deposition. In addition, the most advanced Reynolds stress models (RSM), which solve for the Reynolds stresses individually and perform better in most of the flow domain, are used along with the modified EIM to predict the particle deposition.

LES, which calculates the large-scale structures explicitly, is used in the present work as a verification tool for the RANS/EIM particle simulation. LES permits for more accurate accounting of particle-turbulence interaction because major portion of the flow is simulated without any models.

This chapter describes the particle behavior and its equation of motion. It also presents the governing equations that are used for RANS along with their models. In addition, it describes the methodology of particle modeling in the RANS approach in conjunction with classical and modified EIM. Moreover, the in-house tracking code is described in detail. The details of exporting the flow field and the connectivity data, which are required to run the tracking code, are presented. Finally, the governing equations for LES and the methodology of particle modeling are detailed. 


\subsection{Particle Motion}

\subsubsection{Particle Equation of Motion}

The equation of motion governing the trajectory of a particle is Newton's second law

$$
m \frac{d V}{d t}=\mathrm{F}
$$

where $V$ is the velocity of the particle and $\mathrm{F}$ is the total external force exerted on the particle. Several developments for the particle equation of motion have been proposed by Basset (1961), Tchen (1947) and Corrsin and Lumley (1956) who modified Tchen's equation by adding a term for turbulence pressure gradients. Maxey and Riley (1983) suggested an equation of motion for a small sphere with relative motion of low Reynolds number. Mei (1996) proposed an equation of particle motion corresponding to

$$
\begin{aligned}
\frac{4}{3} \pi a^{3} \rho_{p} \frac{d V}{d t}= & \frac{4}{3} \pi a^{3}\left(\rho_{p}-\rho_{f}\right) g+6 \pi \mu a \phi(U-V)+6 \pi \mu a \int_{t_{0}}^{t} K(t-\tau) \frac{d(U-V)}{d \tau} d \tau \\
& +\frac{2}{3} \pi a^{3} \rho_{f}\left(\frac{\mathrm{DU}}{\mathrm{Dt}}-\frac{d V}{d t}\right)+\frac{4}{3} \pi a^{3} \rho_{f} \frac{D U}{D t}
\end{aligned}
$$

where $\rho_{p}$ and $\rho_{f}$ are the densities of the particle and fluid, respectively. The velocities of the particle and the fluid are, respectively, $V$ and $U$. The particle radius is given as $a=\frac{d_{p}}{2}$, where $d_{p}$ is the particle diameter. Here, $t_{o}$ is the starting time and $\mu$ is the fluid viscosity. 
In Equation (3.2), the term on the left hand side is a summation of the forces acting on the particle expressed in terms of the particle acceleration. The first term on the right hand side is the gravitational acceleration force. The second term is the drag force, which is also known as the Stokes' linear resistance that arises because of the relative velocity. The third term is the Basset force, which accounts for the deviation in the flow pattern from steady state. It is also known as the history term. The fourth term is the added mass or the inertia of the virtual mass force which is caused by the pressure resulting from the relative accelerated motion. The fifth term is the force that results due to the pressure gradient in the fluid surrounding the particle, which is caused by the fluid acceleration. Kim et al. (1998) developed an equation similar to that of Mei (1996).

Except for the drag and the gravitational acceleration forces, all other terms in Equation (3.2) are only significant when the fluid density is comparable to, or greater than, the particle density (Finlay, 2001). Therefore, by considering particles of $5 \mu \mathrm{m}$ in diameter with higher densities than the fluid (i.e., $\rho_{f} / \rho_{p}<<1$ ), Equation (3.2) can be simplified to the following form

$$
\frac{4}{3} \pi a^{3} \rho_{p} \frac{d V}{d t}=\frac{4}{3} \pi a^{3}\left(\rho_{p}-\rho_{f}\right) g+6 \pi \mu a \phi(U-V)
$$

By introducing the particle diameter $d_{p}$ and rearranging Equation (3.3), the following three dimensional Lagrangian equations that describe the motion of particles in the computational domain of the impinging jet are used (Kim et al., 1998; Finlay, 2001; Crowe et al., 1998; Barton, 1995)

$$
\frac{d u_{p}}{d t}=\frac{\left(u_{f}-u_{p}\right)}{\tau_{p} / \phi}
$$




$$
\begin{aligned}
& \frac{d v_{p}}{d t}=\frac{\left(v_{f}-v_{p}\right)}{\tau_{p} / \phi}, \\
& \frac{d w_{p}}{d t}=\frac{\left(w_{f}-w_{p}\right)}{\tau_{p} / \phi}-g\left(1-\frac{\rho_{f}}{\rho_{p}}\right)
\end{aligned}
$$

Where, $\tau_{p}$, the particle relaxation time, which describes the response time of the particles to the changes in the fluid phase motion, is given as

$$
\tau_{p}=\frac{\rho_{p} d_{p}^{2}}{18 \mu}
$$

and the correction factor (Mei, 1996) is

$\phi=1+0.15 \operatorname{Re}_{p}^{0.687}$

$\operatorname{Re}_{p}$ is the particle Reynolds number.

\subsection{Reynolds Averaged Navier Stokes (RANS) Equations}

In RANS, the flow variables (such as velocity and pressure) are divided into a statistically averaged (mean) component and a fluctuation component. For example, the

instantaneous fluid velocity $u_{i}$ can be divided into an averaged, $U_{i}=\frac{1}{T} \int_{t}^{t+T} u_{i} d t$, and a fluctuating component $u_{i}^{\prime}$, such that $u_{i}=U_{i}+u_{i}^{\prime}$. The mean velocity field is obtained by solving the continuity and RANS equations, whereas the fluctuations of the fluid variables need to be modeled. The time average continuity equation and Reynolds averaged Navier Stokes (RANS) equations are given as follows

$$
\frac{\partial \rho}{\partial t}+\rho \frac{\partial U_{i}}{\partial x_{i}}=0
$$


$\rho \frac{\partial U_{i}}{\partial t}+\rho U_{j} \frac{\partial U_{i}}{\partial x_{j}}=-\frac{\partial P}{\partial x_{i}}+\frac{\partial}{\partial x_{j}}\left(2 \mu S_{i j}-\rho \overline{u_{i}^{\prime} u_{j}^{\prime}}\right)$

Simulations using RANS equations require less memory and computational power than other numerical methods such as LES and DNS. However, all scales of the turbulence field are being modeled. Moreover, the averaging procedure produces additional unknown quantities containing products of fluctuating quantities called Reynolds stress given as $-\rho \overline{u_{i}^{\prime} u_{j}^{\prime}}$. For three dimensional flows, we have ten unknown mean flow properties (one pressure, three velocity components and six Reynolds stresses). The number of equations is four including continuity and three components of the Navier Stokes equations. This means the system is undetermined. Therefore, the Reynolds stresses are needed to be modeled by additional equations of known quantities in order to obtain the closure, i.e., an adequate number of equations required for all the unknowns. In order to model the Reynolds stresses, a turbulence model is required. The Reynolds stress can be determined by a turbulence model, either via the turbulent viscosity hypothesis (i.e., eddy viscosity approximation) or more directly from modeled Reynolds stress transport equations. In the present research, the two approaches (i.e., eddy viscosity approximation and the Reynolds stress transport equations) are used to model the Reynolds stress. The two approaches are described in the following subsections. 


\subsubsection{Eddy viscosity model}

In this type of model, the Reynolds stress is computed using eddy viscosity approximation (Pope, 2000), which relates the Reynolds stress to the velocity gradients and the eddy viscosity. The eddy viscosity is obtained from two transport equations, which describe the transport of two scalars (the turbulent kinetic energy $\mathrm{k}$ and its dissipation $\varepsilon$ or the turbulent frequency $\omega$ ). Several models, based on the eddy viscosity hypothesis, are used to model the Reynolds stress, which results due to the averaging procedure. In the current research, the shear stress transport (SST) model is employed using CFX 12.0 (Ansys Inc.) software.

\subsubsection{Shear stress transport (SST) model}

To capture the flow properties accurately, the SST turbulence model is adopted. The SST turbulent model (Menter, 1994) is designed to yield the best behavior of the k- $\omega$ model and the standard k- $\varepsilon$ model. The k- $\omega$ model is used to predict the most turbulent flows because of its reasonable accuracy, robustness and the near wall treatment. On the other hand, away from the wall, i.e., in free shear layers the standard k- $\varepsilon$ model predicts the shear flows more accurately because of the freestream independence of the k- $\varepsilon$ model in the outer part of the boundary layer. However, these two equation models are unable to correctly predict the onset and amount of separation in adverse pressure gradient flows because they lead to overprediction of the turbulence kinetic energy and hence yield large Reynolds stress. Therefore, to resolve this problem, the SST model, which combines the original k- $\omega$ model near the wall and the standard k- $\varepsilon$ model in the free shear layers away from the wall, was developed. In the SST formulation, the k- $\omega$ formulation is utilized 
near the wall and switches to the k- $\varepsilon$ behavior in the free stream. In this model the definition of the eddy viscosity is adjusted to account for the transport of the turbulent shear stress and thus gives highly accurate predictions of the start and the amount of flow separation under adverse pressure gradients. The governing equations for SST model are described in Appendix A.

\subsubsection{Reynolds stress model (RSM)}

Another approach used in this work is the Reynolds stress model (RSM). In this model, the transport equation for the Reynolds stress, $-\rho \overline{u_{i}^{\prime} u_{j}^{\prime}}$, in the fluid is solved without need for the eddy viscosity assumption, which is used in the SST model. Beside the Reynolds stress equation, another equation for the turbulent frequency $\omega$ or turbulent dissipation $\varepsilon$ has to be added to determine the length scale of the turbulence. The RSM is more suited to complex flows because it accounts for the effects of a strong streamline curvature, sudden changes in the strain rate, and secondary flows (Wilcox, 2006).

Two types of Reynolds stress models: Omega Reynolds stress model (RSM- $\omega$ ) and Baseline Reynolds stress model (RSM-BSL) were utilized using CFX 12.0 (Ansys Inc.) software. Both models are based on the $\omega$-equation that allows for a more accurate near wall treatment with an automatic switch from a wall function to a low-Reynolds number formulation based on the grid spacing. However, since their performances were almost similar, the RSM-BSL model was considered in the present research. The governing equations for RSM- $\omega$ and RSM-BSL models are described in Appendix A. 


\subsection{Particle modeling in RANS approach}

\subsubsection{Eddy interaction model (EIM)}

The Eddy interaction model (EIM) is a Lagrangian simulation method used to numerically track liquid or solid particles released in turbulent flows. This type of model is described by using the term "random walk" (MacInnes and Bracco, 1992).

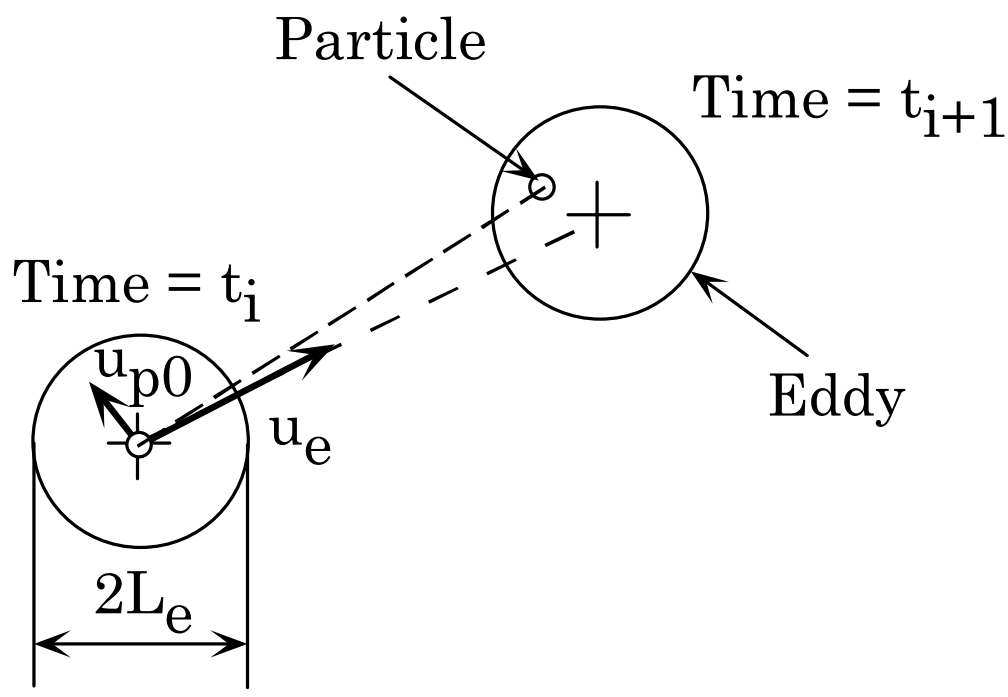

Figure 3.1. Eddy interaction model.

In EIM, one particle is allowed to interact successively with various eddies. Each eddy has a characteristic lifetime, length and velocity scales that are obtained from the primary flow calculation results. The end of the interaction between the particle and one eddy occurs when the lifetime of the eddy is over or when the particle crosses the eddy as shown in Figure 3.1. At this instant, a new interaction for the particle with a new eddy is started. The particle will have another trajectory according to its equation of motion (Kim et al., 1998; Mei, 1996).

When the particle enters the eddy, the local fluctuating velocity at the particle location is added to the local mean velocity to obtain the instantaneous fluid velocity used 
in the particle equation of motion. The local fluctuating velocities are obtained by multiplying the root mean square (RMS) fluid fluctuating velocities by random numbers generated from a Gaussian probability density function of zero mean and unit standard deviation at the start of one eddy-particle interaction. In order to obtain velocity or deposition statistics, hundreds of particles must be released into the flow.

The characteristic eddy scales should be determined to predict the particle motion. The eddy scales can be determined from isotropic turbulence flow field. Shuen et al. (1983a), based on method of Gosman and Ionannides (1981), simulated the particle dispersion in a turbulent round jet using the k- $\varepsilon$ turbulence model and the following velocity, length, and time scales of fluid eddies:

$$
\begin{aligned}
& u_{e}=(2 k / 3)^{0.5}, \\
& L_{e}=\left(C_{\mu}\right)^{3 / 4} \frac{k^{3 / 2}}{\varepsilon}, \\
& \tau_{e}=\frac{L_{e}}{(2 k / 3)^{0.5}}
\end{aligned}
$$

In specifying the eddy lifetime, it is important to consider the Lagrangian time scale $\tau_{L}$. Kallio and Reeks (1989), in their numerical simulations, estimated $\tau_{L}$ using an isotropic expression that depends on the turbulence kinetic energy $k$ and the dissipation rate $\varepsilon$. In determining the eddy lifetime scale the constant lifetime scheme of Hutchinson et al. (1971) and Govan et al. (1989) is used such that

$$
\tau_{e}=2 \tau_{L}
$$

Wang and Stock (1992) and Graham and James (1996) noted that using the constant eddy lifetime scheme ensures dispersion consistency. Shuen et al. (1983a) used 
the constant eddy lifetime scheme in their simulation. Other studies, such as Kallio and Reeks (1989), Tian and Ahmadi (2007), and Zamora and Kaiser (2011), estimated the eddy lifetime by randomizing the lifetime scale from an exponential probability density function. However, Graham and James (1996) and Tian and Ahmadi (2007) indicated that the constant eddy lifetime scheme is preferred over the exponential distribution scheme because it reduces the computational cost. The constant eddy lifetime scheme, as given by Equation (3.14), is used in the present study.

In the isotropic turbulent flow field, the eddy life time scale is obtained by

$$
\tau_{e}=0.2 \frac{k}{\varepsilon}
$$

and the eddy velocity scales are computed from

$$
u_{e}=\left(\frac{2 k}{3}\right)^{0.5}
$$

where $k$ is the turbulence kinetic energy. In the anisotropic turbulent flows, $\tau_{L}$ is computed using the normal-to-wall turbulence mean square fluctuation (Tian and Ahmadi, 2007) as

$$
\tau_{L}=C \frac{\overline{w^{\prime 2}}}{\varepsilon}
$$

in order to determine the eddy lifetime scale from Equation (3.14). In the present study, $C$ is the Lagrangian time scale constant and $\overline{w^{\prime 2}}$ the normal-to-wall turbulence mean square fluctuation. In the literature, there is no specific value for $C$. In the present study, $C=0.1$ 
was used based on a sensitivity analysis of the constant to the particle deposition results as will be shown in Section 5.4. The eddy velocity scale in anisotropic turbulent flow is calculated as

$$
u_{e}=\left(\overline{u_{i}^{12}}\right)^{0.5}
$$

where $\left(\overline{u_{i}^{12}}\right)^{0.5}$ is the RMS local fluctuating velocity in the $\mathrm{i}^{\text {th }}$ direction.

In the present study, Equations (3.15) and (3.16) were used to calculate the eddy lifetime and the velocity scales when the primary flow phase is computed by eddy viscosity models (SST model). For the RSM models, Equations (3.17) and (3.18) were used to calculate the eddy lifetime and the velocity scales. Since the particles that were used in the present study are relatively small $(5 \mu \mathrm{m})$, crossing trajectory effects were not relevant and the length scales can be neglected (Matida et al., 2000).

The fluid velocity components in Equations (3.4)-(3.6) are instantaneous quantities composed of the mean part and the fluctuation part, for example,

$$
u_{f}=U_{f}+u_{f}^{\prime}
$$

The local fluctuation velocities of the eddy are generated at the start of each interaction by multiplying the RMS fluctuation velocities $\left(u_{r m s}^{\prime}, v_{r m s}^{\prime}, w_{r m s}^{\prime}\right)$ obtained from Equations (3.16) and (3.18) by the random number $\left(N_{u}, N_{v}, N_{w}\right)$ such that

$$
\begin{aligned}
& u_{f}^{\prime}=N_{u} u_{r m s}^{\prime} \\
& v_{f}^{\prime}=N_{v} v_{r m s}^{\prime} \\
& w_{f}^{\prime}=N_{w} w_{r m s}^{\prime}
\end{aligned}
$$


The random numbers are generated from a Gaussian probability density function of zero mean and unity standard deviation at the start of one eddy-particle interaction. In this study, there are two versions of EIM that will be used. They are the classical EIM and the modified EIM. The next two subsections will detail those two versions of EIM.

\subsubsection{Classical eddy interaction model}

This version of EIM is based on Gosman and Ioannides (1981) model along with the length, time and velocity scales of fluid eddies Equations (3.11)-(3.13). In this type, during the eddy-particle interaction, the mean fluid velocity $U_{f}$ in Equation (3.19) varies according to the particle position. However, RMS fluid fluctuating velocity (eddy velocity) is constant during the eddy-particle interaction. This version of EIM is used in CFX 12.0 (Ansys Inc.) tracking code.

\subsubsection{Modified eddy interaction model}

In this type, at the start of an eddy-particle interaction, the random number was generated and kept constant during the eddy-particle interaction as the classical EIM. However, both the local mean velocity and the fluctuating velocity were allowed to vary during the interaction, as the particle is within the eddy. This type of EIM was adopted in an inhouse tracking code, which is developed to track the particles in impinging jet flow. The simulation of the turbulent fluctuations including the near-wall corrections and particle trajectory analysis were performed using the in-house tracking code. The near-wall corrections and how they were incorporated in the tracking code will be described in Subsections 4.9.2 and 5.3.2. The next section describes in more details the developed inhouse tracking code. 


\subsubsection{Particle Tracking Code}

In order to track the particles using the modified EIM, the in-house particle tracking code (using FORTRAN language source) was developed in the present study. Moreover, this tracking code allows us to improve the particle deposition in the impinging jet flow by adding near-wall corrections as will be described later.

This code predicts the trajectory of the particles released numerically in the flow field, which is solved using CFX 12.0 (Ansys, Inc.). During the tracking process, each particle travels from one hexahedric element to another in the computational domain. Figure 3.2 shows a schematic of the hexahedric element. As the particle is tracked in the computational domain, the code transforms the actual hexahedric elements in the global $x$ $y z$ system used by CFX into isoperimetric elements in the local $a b c$ system as shown in Figure 3.2. A single particle is tracked by solving the particle equations of motion (Equations 3.4-3.6). The above equations are integrated twice inside the hexahedric element using fourth order Runge-Kutta method. The first integration is to obtain the velocity of the particle and the second integration is to obtain the position of the particle. The integration continues until the value of one of the local coordinate variables (i.e., $a$, $b$, or $c$ ) becomes smaller than -1 or greater than 1 , which implies that the particle enters a new hexahedric element.

In order to calculate the particle trajectories, the flow variables such as the mean velocity and other turbulence quantities should be obtained at the particle position. The particle position in the Lagrangian approach was obtained by interpolating the actual position of the particle in the Eulerian approach (Cartesian coordinates, $x y z$ ) as follows 


$$
x=\sum_{i=1}^{8} N_{i} x_{i}, \quad \mathrm{y}=\sum_{i=1}^{8} N_{i} y_{i}, \quad z=\sum_{i=1}^{8} N_{i} z_{i}
$$

where

$$
N_{(i=1-8)}=\frac{1}{8}\left[1+\left(a_{i}\right) a\right]\left[1+\left(b_{i}\right) b\right]\left[1+\left(c_{i}\right) c\right]
$$

such that $i=1,2, \ldots \ldots, 8$ indicating the vertices of the hexahedric element and $a_{i}, b_{i}$ and $c_{i}$ having accordingly values of -1 or 1 . The main fluid flow variables were also transformed from the Eulerian domain to the Lagrangian domain by using Trilinear interpolation to obtain their values at the particle position. Trilinear interpolation of the variables $\phi$ such as fluid mean velocity, turbulence kinetic energy, Reynolds stresses and dissipation can be obtained by

$$
\phi(x, y, z)=\left[N_{1} N_{2} \ldots \ldots N_{8}\right]\left[\begin{array}{c}
\phi_{1} \\
\phi_{2} \\
\ldots \\
\phi_{8}
\end{array}\right]
$$

where $\phi_{i}(i=1-8)$ are nodal values of these variables.

The time step that is used in the integration of the particle equation of motion was an adaptive time step and set at each start of an eddy-particle interaction, or when the particle enters a new element. The time step was chosen to be the minimum of one fifth of the eddy lifetime or one fifth of the minimum side length of the local element divided by the particle local velocity, that is

$$
\Delta t=\min \left(\frac{\tau_{e}}{5}, \frac{1}{5} \frac{L_{\min }}{\left(u_{p}^{2}+v_{p}^{2}+w_{p}^{2}\right)^{0.5}}\right)
$$




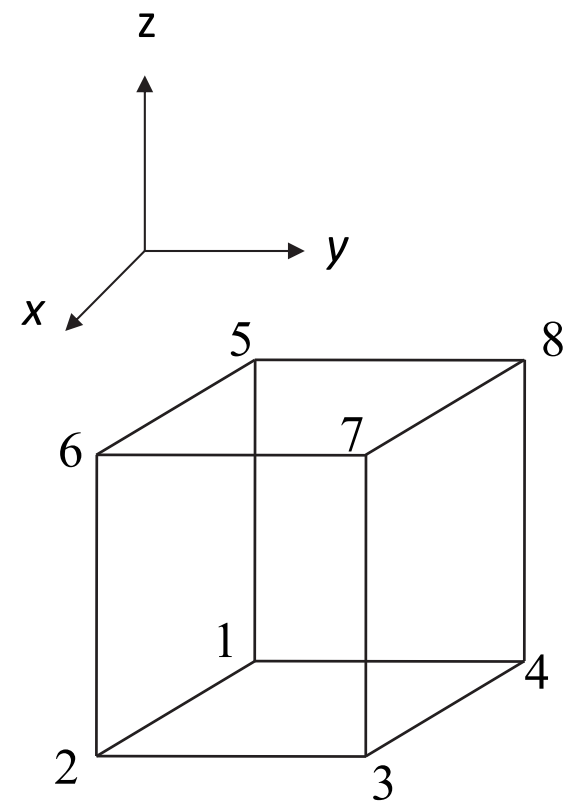

(a)

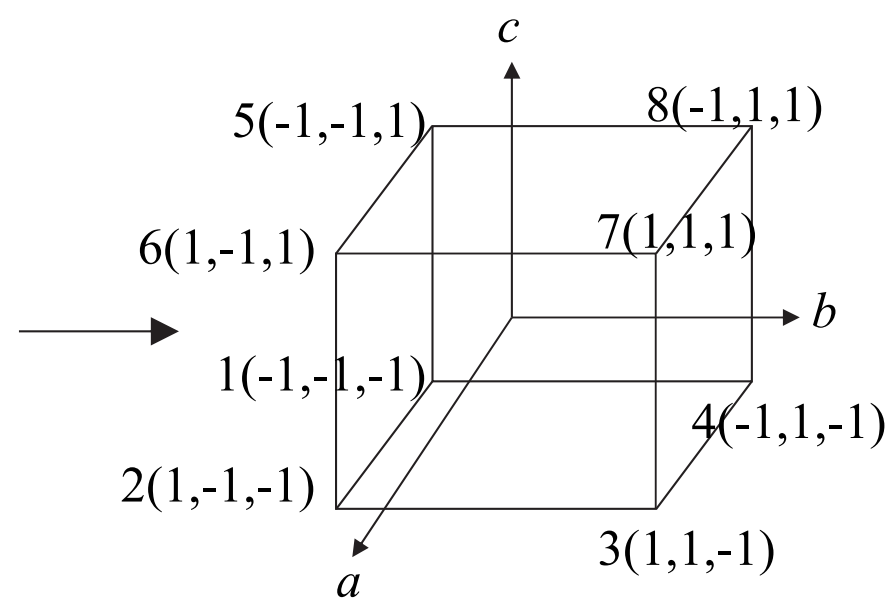

(b)

Figure 3.2. Transformation of the hexahedric element: (a) in $x y z$ system, and (b) in a b c system.

where $\tau_{e}$ is the eddy lifetime and $u_{p}, v_{p}$ and $w_{p}$ are the local particle velocities along $x$, $y$, and $z$, respectively. The particles are released individually and their initial positions are assigned randomly. Their initial velocities were made equal to the fluid velocity. The particles were assumed to deposit and stick to the impingement wall as the centroids of the particles reach a distance from the wall equivalent to the radius of the particle. Near the wall the eddy lifetime was made constant and equal to the minimum Lagrangian time scale (Kallio and Reeks, 1989). This approach is efficient in circumventing the problem that occurs near the walls where the eddy lifetimes asymptotes to infinitesimal values which increases the computational time. 


\subsubsection{Details of exporting the flow field and connectivity data}

To track the particles using the modified EIM through the in-house tracking code, the flow field results and the computational domains information must be exported and fed to the tracking code. The computational domains that are used in the present study were generated using CFD-ICEM mesh generator. The computational domains are composed of multi-block structures that consist of hexahedral elements. This will be described in Section 4.1. Thus, after the flow field was calculated separately by CFX, the results (including the coordinates and the turbulence quantities) were exported in Fieldview unstructured format. The structured block files, which list the name and the range of all blocks composing the structured mesh, were exported from CFD-ICEM using Multiblock translator. In order to match the grid coordinates that were obtained from the CFX (Fieldview unstructured format) according to the range and nodal order of each block that were obtained from CFD-ICEM, a customized (FORTRAN source) code was built to accomplish this process. Therefore, by using the outputs of this code, the grid coordinates and all the turbulence values, as well as other quantities, such as turbulence kinetic energy and dissipation, were placed in their corresponding nodes for each block. To verify the correct matching of the coordinates and other turbulence quantities, these quantities were post-processed and compared with the corresponding quantities that were post-processed by CFX-post.

The computational domain of the impinging jet is composed of 13 structured multiblocks that involve a large number of elements, as will be described in Section 4.1. Each block has six faces and a coordinate system (orientation). Figure 3.3 demonstrates a schematic of a block with its faces (face 1 to face 6 ) and coordinate (i, j and k indices). 
Since the particles move from one element to another (inside a block) or from a block to another during the tracking process, the connectivity information of these elements and blocks should be known.

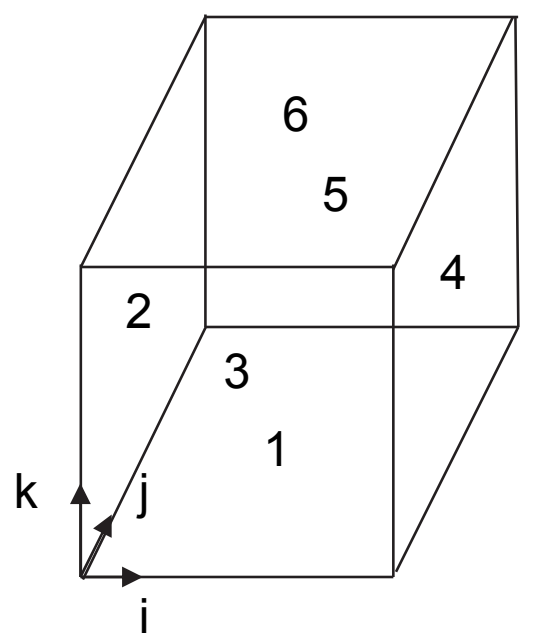

Figure 3.3. Cubic block faces.

The information concerning the connectivity between the blocks and between the blocks and other boundaries (inlet, outlet and walls) was obtained from CFD-ICEM using Multiblock translator. The connectivity information contains the shared faces of the connected blocks and the faces of the blocks that are connected to other boundaries (inlet, outlet and walls). This information lists the start and end of the node range along $\mathrm{i}, \mathrm{j}$ and $\mathrm{k}$ directions. In the present study, a convention used to determine what face of a block is connected to another face of another block was adopted. In this convention, as shown in Figure 3.3, faces 2 and 4 are assigned, respectively; at the start and end of the $i$ index. Faces 3 and 5 are assigned, respectively, at the start and end of the $\mathrm{j}$ index. Faces 1 and 6 are assigned, respectively, at the start and end of the k index. 


\section{Face $1 \quad$ Face 1 (4 orientations)}
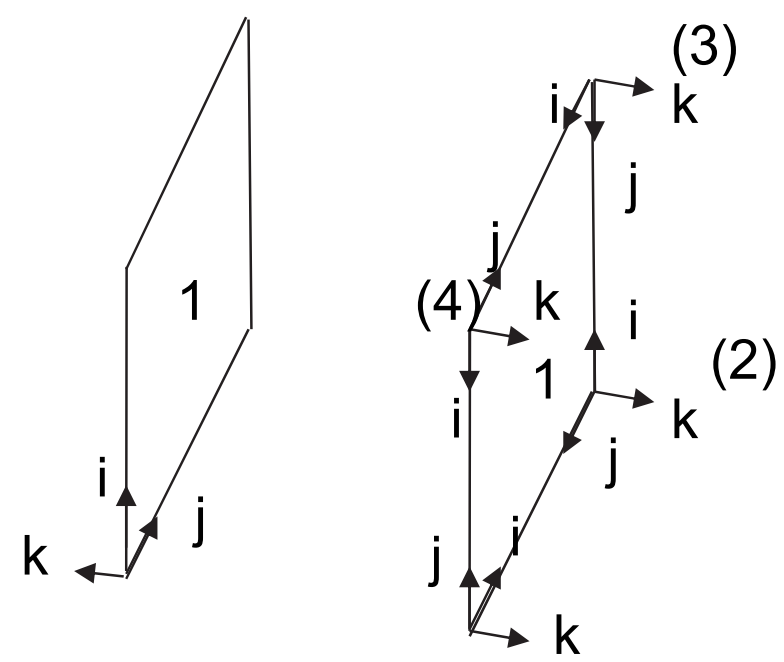

(1)

Figure 3.4. Block orientations.

In determining how a block is connected to another block (new block), it is important to know the coordinate system (orientation) of the new block. This was determined by adopting a convention that uses the right-hand rule. Figure 3.4 describes the adopted convention. When face 1 of arbitrary block is connected to face 1 of another block (new block), there are four possible coordinates orientations for the new block. Thus, based on the start and end of each index (i.e., i and j) of the attached new block, the appropriate orientation can be chosen.

In forming the connectivity between the blocks and other boundaries, each block is indicated by a specific number. The face number and the orientation number for the blocks are determined as mentioned in the above paragraphs. The inlet, outlet and wall boundaries are indicated by the number zero and orientation zero meaning that there are no coordinates. The inlet and outlet boundaries are designated by one face symbolized by 
number "7". The wall boundary is also designated by one face symbolized by number zero " 0 ". Hence, using this information and applying the mentioned conventions in a customized code, the connectivity between the blocks and other boundaries was constructed. Table 3.1 shows an example of the connectivity information, which was obtained from the customized code and can be read into the in-house tracking code. In Table 3.1 , the first row indicates that face $1\left(2^{\text {nd }}\right.$ column $)$ of block $1\left(1^{\text {st }}\right.$ column $)$ is connected to an inlet or outlet boundary because it has face "7". The second row shows that face 2 ( $2^{\text {nd }}$ column) of block $1\left(1^{\text {st }}\right.$ column $)$ is connected to face 4 of block 4 and block 4 has orientation 1 . The third row shows that face $3\left(2^{\text {nd }}\right.$ column $)$ of block $1\left(1^{\text {st }}\right.$ column) is connected to a wall, which is indicated by "0" for its number, face number and the orientation.

Table 3.1. Connectivity of block 1 with other blocks and boundaries.

\begin{tabular}{|c|c|c|c|c|}
\hline $\begin{array}{c}\text { Block } \\
\text { number }\end{array}$ & $\begin{array}{c}\text { Face } \\
\text { number }\end{array}$ & $\begin{array}{c}\text { New block or } \\
\text { boundary } \\
\text { number }\end{array}$ & $\begin{array}{c}\text { Face of new block or } \\
\text { boundary number }\end{array}$ & $\begin{array}{c}\text { Orientation } \\
\text { number }\end{array}$ \\
\hline 1 & 1 & 0 & 7 & 0 \\
\hline 1 & 2 & 4 & 4 & 1 \\
\hline 1 & 3 & 0 & 0 & 0 \\
\hline
\end{tabular}

After the flow field results are exported according to the structured mesh (i.e., node order), they are supplied to the in-house tracking code. Figure 3.5 shows the flow chart of the steps that were performed to make the fluid results and connectivity information available to the tracking code. 


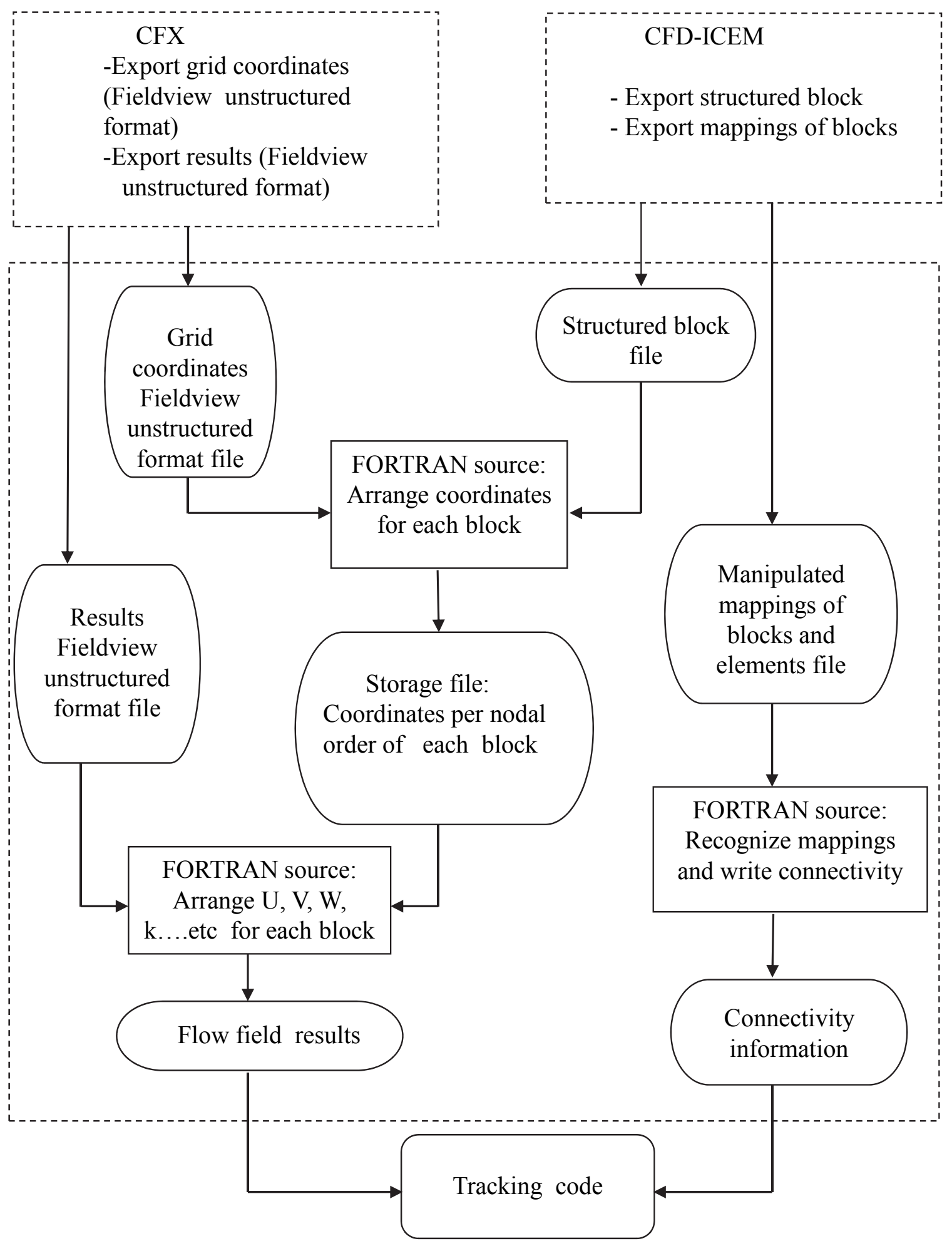

Figure 3.5. Flow chart of the steps of exporting the flow field and connectivity information. 


\subsection{Large Eddy Simulation}

Large eddy simulation (LES) lies, in terms of accuracy and computational requirements, between RANS and DNS approaches. Since the large energy carrying eddies are highly influenced by the boundary conditions, they can be resolved by computation, whereas the small eddies (unresolved scales) are modeled. The large scales of motion are more energetic than the small scales. Thus, their size and strength make them effective transporters of the conserved properties (Piomelli, 2001). LES is a three-dimensional and time dependent approach.

By applying the filtering operation to the Navier-Stokes equations (Piomelli, 2001), the filtered Navier-Stokes equations, which are solved in large eddy simulations, are written in the following form

$$
\begin{aligned}
& \frac{\partial \bar{U}_{i}}{\partial x_{i}}=0 \\
& \frac{\partial \bar{U}_{i}}{\partial t}+\frac{\partial}{\partial x_{j}}\left(\bar{U}_{i} \bar{U}_{j}\right)=-\frac{1}{\rho} \frac{\partial \bar{P}}{\partial x_{i}}-\frac{\partial \tau_{i j}}{\partial x_{j}}+v \frac{\partial^{2} \bar{U}_{i}}{\partial x_{j} \partial x_{j}}
\end{aligned}
$$

where $\tau_{i j}=\bar{U}_{l} \bar{U}_{J}-\bar{U}_{i} \bar{U}_{j}$ is the subgrid scale (SGS) Reynolds stress (Piomelli, 2001). The SGS Reynolds stress is analogous to the Reynolds stress tensor in RANS (Pope, 2000). In RANS, the velocity fluctuations $u_{i}^{\prime}$ (turbulent fluctuations) represent all the turbulent motions, whereas in LES, $u_{i}^{\prime}$ (the small eddies) represents only the small scales (Wagner et el., 2007). Therefore, the implications of this are that the energy in the unresolved scales of LES (small eddies) represents a much smaller portion of the total energy of the 
flow in comparison with the energy in the unresolved scales (turbulent fluctuations) of RANS.

Like RANS, the filtered Equations (3.24) and (3.25) are unclosed. The closure is obtained by modeling the SGS residual stress tensor. In the present study, the SGS residual stress is modeled by using Smagorinsky model. The Smagorinsky model is shown in Appendix B.

\subsection{Particle modeling in LES}

LES offers the advantage of solving the large scale eddies directly while modeling the small scale eddies. This approach is several orders of magnitude more expensive than a RANS simulation and is, therefore, not used routinely in industrial flow simulations. However, it provides much more information than the RANS approach. This means that it gives more details about the flow features in the fluid phase, which interacts strongly with the particulate phase.

In the present research, the frozen domain particle tracking technique to track the particles that are released in the LES computational domain was adopted. By using this technique, the equations of motion of particles (Equations 3.4-3.6) are solved where the filtered velocity (resolved) component was used as the corresponding fluid velocity. In LES, the energy in the subgrid scales (unresolved scales of LES) represents a much smaller fraction of the total flow energy. Therefore, for a high density ratio $\rho_{p} / \rho_{f}$ (Wang and Squires, 1996) and a moderate Reynolds number, as is considered in the present research, in addition to a highly refined mesh (DNS like) of the flow field domain, the subgrid scale contribution to the turbulent kinetic energy is very small 
(Breuer et al., 2006). Moreover, in LES predictions, the particle dispersion is governed mainly by the large-scale motion, such that the particles follow the large scale eddies in their trajectories (Uijttewaal and Oliemans, 1996 and Elghobashi, 1994).

In the frozen domain particle tracking technique, which is available in CFX 12.0, all the particles are released and tracked in single steady-mode iteration without any modeling and using $1 \mu$ s of physical time scale in convergence control (CFX 12.0). In this technique, thousands of particles are released in a frozen LES computational domain and fully tracked in one iteration and then the deposition statistics are recorded. 


\section{Chapter 4: Results for RANS-SST with EIM}

This chapter presents RANS SST/EIM, where the SST model is formulated on the basis of eddy viscosity assumption, in order to predict the particle deposition in impinging jet flow. Two versions of EIM, classical EIM (used through CFX code) and modified EIM (using the developed tracking code) were used to predict the deposition by "turbulent tracking" (without any corrections) over the impingement wall. Results from turbulence eddy ratio correction in conjunction with classical EIM were compared with the results obtained by turbulent tracking. The near-wall correction function (Van Driest, 1956), based on the impinging jet flow characteristics, was presented. The results using the nearwall correction, which was incorporated into the tracking code and implemented through the modified EIM, are presented and compared with turbulent tracking. The deposition results are validated by comparing with the impinging jet deposition measurements data of Burwash et al. (2006). The accuracy of the impinging jet flow field simulations that are conducted under the experimental conditions of Burwash et al. (2006) and Cooper et al. (1993) are validated by comparing with the experiment of Cooper et al. (1993) and the simulation of Kubacki and Dick (2010b).

In addition, the particle deposition patterns over the impingement wall are presented and compared. Several effects on the particle deposition are reported and tested: the effect of the number of particles and also the effect of the nozzle-to-surface distance. 


\subsection{Computational domain and numerical parameters}

Before presenting the computational domain and the numerical parameters, the experiment of Burwash et al. (2006), which is used to validate the simulated deposition results, is described. Figure 4.1 shows a schematic of the confined jet showing a deposition chamber including the nozzle attached to the nozzle plate and deposition surface. The nozzle is a contraction nozzle with diameter of $D=15 \mathrm{~mm}$. The deposition chamber as shown in the figure consists of a deposition surface with diameter of $440 \mathrm{~mm}$ and a nozzle plate with diameter of $370 \mathrm{~mm}$. The nozzle plate was attached to the nozzle to guide the flow and to maintain the impinging jet flow and the deposition surface from the flow re-entrainment. The deposition surface is a circular sheet of plastic secured to the bottom of the deposition chamber. The particles used in their experiment were solid polystyrene microspheres particles with size of $5 \mu \mathrm{m}$. These aerosol particles are mixed with dry air and then moved into a secondary mixing chamber, which is attached to the nozzle to permit mounting of the nozzle and to allow the nozzle-to-surface distance to be set and changed easily. The flow rate at the nozzle exit was $111 \mathrm{~L} / \mathrm{min}$ and Reynolds number of $\operatorname{Re}=10000\left(U_{j}=10.5 \mathrm{~m} / \mathrm{s}\right)$ was maintained at the nozzle exit.

In their experiment, the authors used a microscope-camera system and an image processor to analyze the deposition patterns of the particles. By using the microscopecamera system along with the suitable laser, which is used for particle excitation (short wavelength UV light), several images of the deposition patterns on the deposition surface were taken. Then, the images are processed using the image processor identification capabilities, which assign a particle deposition density for the individual images of the 
deposition surface. This was obtained by knowing the total number of the particles in the images (obtained from image processor) and the dimensions of the surface captured in the image, which is obtained through the calibration of the microscope-camera system. A profile for the deposition density of the particles from the stagnation point was compiled by taking several images at particular distances from the center of the deposition pattern, which is determined by identifying the point on the pattern directly below the center of the nozzle. The surface was imaged outward every $2 \mathrm{~mm}$ successively from the stagnation point until the deposition vanishes. In the experiment, the deposition was measured for three cases of nozzle-to-surface, $L / D$, distances of 2, 4 and 6 . Each case of $L / D$ was repeated three times and one radial ray from the stagnation point outward was analyzed for each repetition. The deposition density curves for the three cases are plotted versus the radial distance outward from the stagnation point (as shown in Figure 2.3). Each point in the curves is the average of three repeats. The standard error in addition to the error associated with the image processing is $\pm 11 \%$. The error in the nondimensional distance due to the standard error and the error in the location of the stagnation point is \pm 0.067 ( $\pm 1 \mathrm{~mm})$ (Burwash et al., 2006).

The dimensions of the computational domain (Figure 4.1) were made based on the experiment of Burwash et al. (2006). Indeed, the flow parameters, i.e., inlet velocity and Reynolds number were made the same as in their experiment. The height $L$, the distance between the nozzle and the deposition surface, varies by 30,60 and $90 \mathrm{~mm}$ leading to three nozzle-to-surface distance cases of $L / D=2,4$ and 6 , where $D$ is the nozzle diameter. 


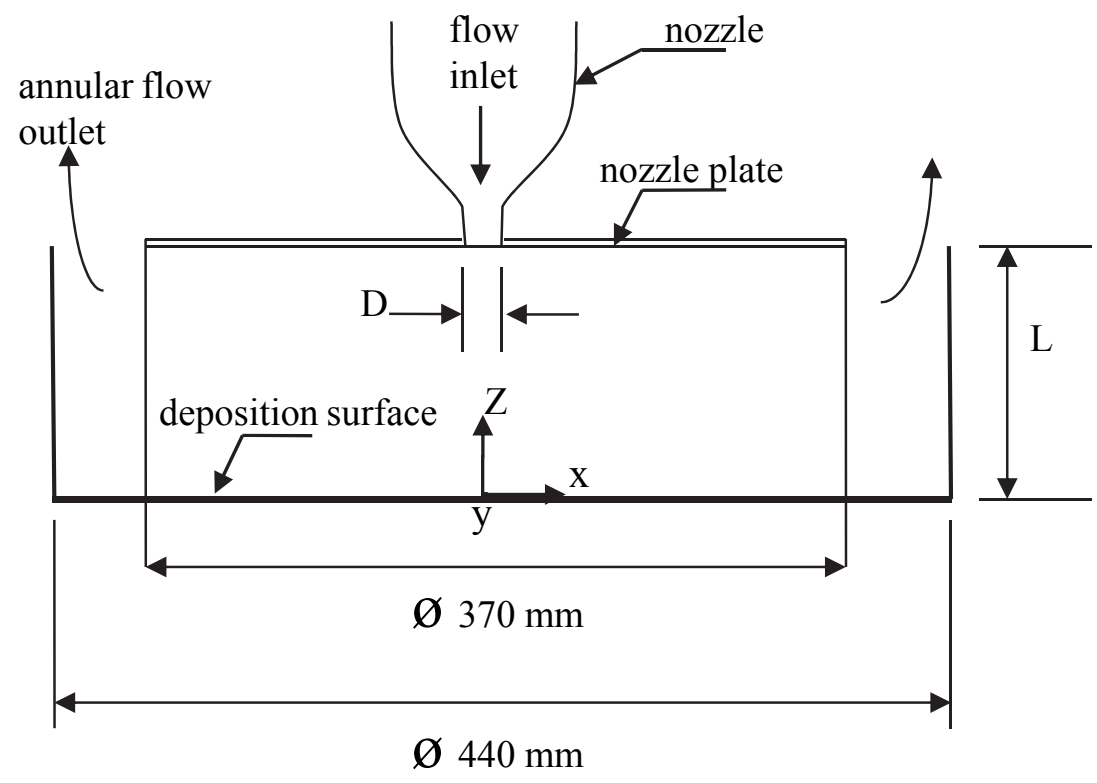

Figure 4.1. Schematic of the computational domain of the impinging jet based on the experiment of Burwash et al. (2006).

The nozzle-to-surface distance is important parameter in impinging jet flow. Its effect on the fluid phase was widely investigated in the previous literature as in Cooper et al. (1993). One of the objectives of the present research is to investigate the influence of this parameter on the particle deposition using three cases of $L / D=2,4$ and 6 . Therefore, the choice of these cases was made in order to understand the deposition behavior through numerical prediction as the nozzle-to-surface distance changes. Also, this choice was made in accordance with the available measurements of Burwash et al. (2006) for which these measurements are used to validate the present simulations.

The computational domains for the three nozzle-to-surface distances, $L / D=2,4$ and 6, were constructed using CFD-ICEM (Ansys Inc.) mesh generator code. Each mesh 
of these cases is composed of structured multi-block mesh. Each mesh consists of 13 blocks. For each case, the grid points were accumulated at the bottom, top and side walls in order to fulfill the condition $\mathrm{y}+<3$ (Kubacki and Dick, 2010a). In addition, grid points were accumulated in the shear layer of the jet since these are the regions of high velocity gradients. Also, the grid points were clustered in the central region of the geometry (from the inlet up to the stagnation point) and towards the impingement wall (near-wall region) as shown in Figure 4.2. Figure 4.2a shows a top view of the computational domain where the grid points were accumulated in the central region of the domain, and Figure $4.2 \mathrm{~b}$ shows a cluster of the grid points from the inlet up to the stagnation point and towards the impingement wall. The near-wall region is the region of interest where the deposition occurs. This region is crucial for the deposition prediction using near-wall correction. Therefore, it was necessary to impose low enough values of $\mathrm{y}+$ for accurate predictions of deposition. The values of $y+$ at the first grid point over the impingement wall were in range of about $0.2 \leq y^{+} \leq 1.4$. Discontinuities in node spacing at the boundaries were avoided in order to have smooth connections.

The numerical accuracy can be enhanced by using a highly refined mesh. Four grid sizes of $248,676,497,408,761,978$ and $1,007,073$ nodes were used to perform grid convergence analysis for $L / D=2$. Based on the adequate grid size that was chosen for $L / D=2$, grids for $L / D=4$ and 6 were established. This way was adopted based on previous numerical simulations for impinging jet flow (Craft et al., 1993). For $L / D=4$ 


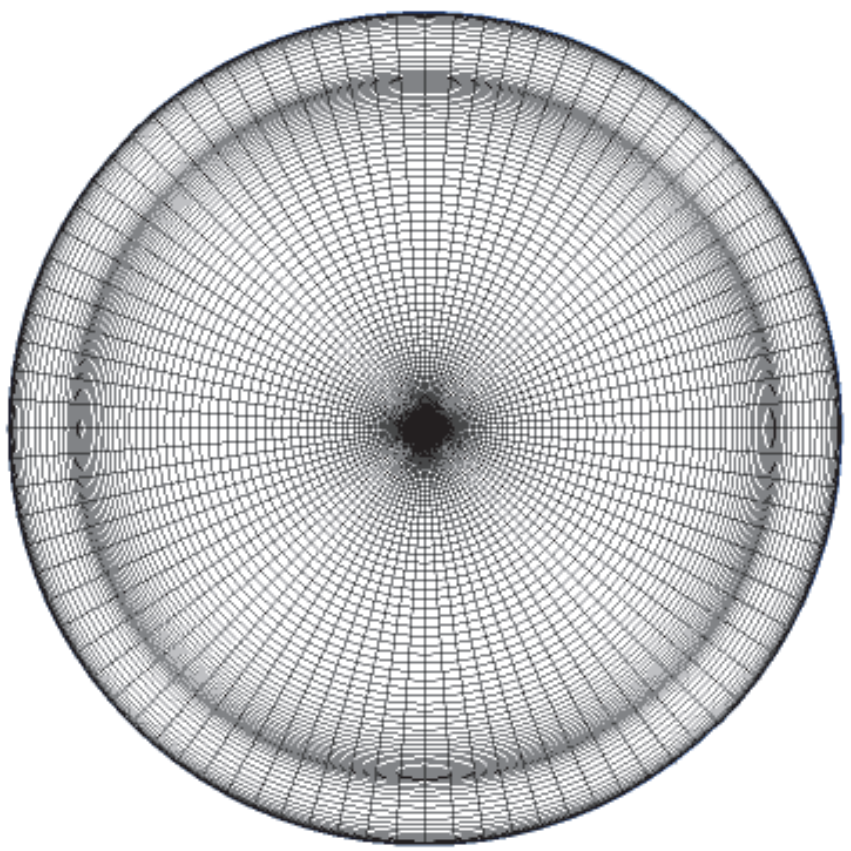

a) Top view of the computational domain.
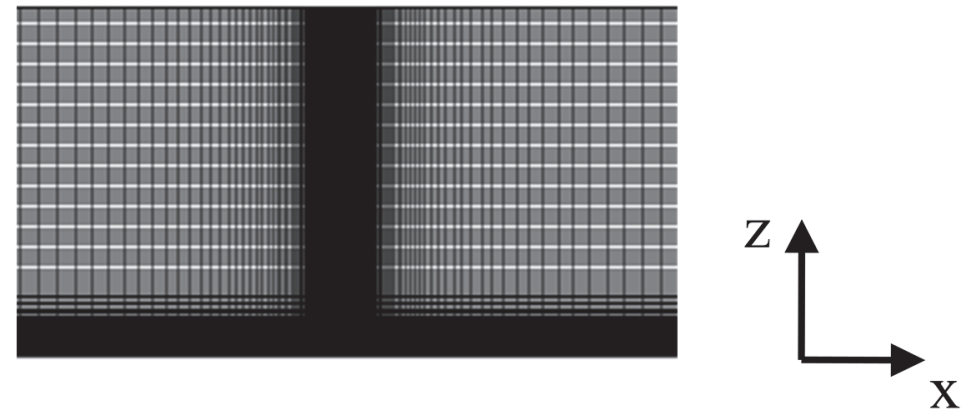

b) Zoom of the normal-to-wall mesh in $x-z$ plane.

Figure 4.2. Grid of the computational domain of the impinging jet used for RANS/EIM calculations for: (a) Top view of the computational domain, and (b) Zoom of the normal-to-wall mesh in $x-z$ plane $(y=0)$. 
and 6 , the nodes along other directions ( $x$ and $y$ axes) were kept the same as the chosen grid for $L / D=2$. However, along the $z$ direction (normal-to-wall), the number of nodes was increased due to the increase in the nozzle-to-surface distance as shown in Table 4.1. Also, the grid density was higher near the wall and coarsened in the outward direction with a grid expansion factor of 1.01 for $L / D=2,4$ and 6 as presented in Table 4.1.

Table 4.1. Details of computational domains.

\begin{tabular}{|c|c|c|c|}
\hline$L / D$ case & $\begin{array}{c}\text { Number of nodes along } \\
\text { normal-to-wall direction }\end{array}$ & $\begin{array}{c}\text { Expansion } \\
\text { factor }\end{array}$ & $\begin{array}{c}\text { Total number of } \\
\text { nodes }\end{array}$ \\
\hline 2 & 74 & 1.01 & 761,978 \\
\hline 4 & 146 & 1.01 & $1,503,362$ \\
\hline 6 & 219 & 1.01 & $2,555,043$ \\
\hline
\end{tabular}

The numerical simulations of the primary fluid phase were carried out using CFX 12.0 (Ansys Inc.) software. Airflow at $25 \mathrm{C}^{\circ}$, incompressible and steady state fluid flow was assumed in the solution. The boundary conditions were set as follows. The inlet conditions consist of the Top-Hat profile (i.e., constant inlet velocity $U_{j}=10.5 \mathrm{~m} / \mathrm{s}$ ) giving steady state flow rate of $111 \mathrm{~L} / \mathrm{min}$, a turbulence intensity of $5 \%$ of the mean velocity and turbulence length scale of $10 \%$ of the inlet diameter. These inlet values of turbulence intensity and length scale were chosen based on the experiment of Burwash et al. (2006), who used a contraction nozzle exit and had turbulent intensity of $5 \%$ of the mean velocity at the nozzle exit. The Reynolds number based on the inlet diameter is Re $=\rho U_{j} D / \mu=10^{4}$, where the air density is $\rho=1.18 \mathrm{~kg} / \mathrm{m}^{3}$, the nozzle diameter is $D=15$ $\mathrm{mm}$ and $\mu$ is the viscosity of the fluid $\left(\mu=1.824 \times 10^{-5} \mathrm{~kg} / \mathrm{m} . \mathrm{s}\right)$. The standard no slip 
boundary condition was applied to the walls and a constant zero gage pressure opening boundary was used at the outlet of the computational domain.

In the present simulation, the particulate phase is simulated using CFX tracking code, where classical EIM is used, and the in-house tracking code, where modified EIM is used. The particles were modeled to be solid, having a diameter of $5 \mu \mathrm{m}$ and a density of $1050 \mathrm{~kg} / \mathrm{m}^{3}$. The flow rate was specified as $2.0 \times 10^{-9} \mathrm{~kg} / \mathrm{s}$. One-way coupling was used to govern the particle-fluid interaction. A number of 10,000 particles were released in the computational domain. The particle Stokes number is defined as

$$
S t k=\frac{\rho_{p} C_{c} U_{j} d_{p}^{2}}{9 \mu D}
$$

where $\rho_{p}$ is the density $\left(\rho_{p}=1050 \mathrm{~kg} / \mathrm{m}^{3}\right)$ and $d_{p}$ is the diameter $\left(d_{p}=5 \times 10^{-6} \mathrm{~m}\right)$ of the particles. $C_{c}$ is the slip factor and assumed to be approximately equal to one. The Stokes number investigated in this study is $S t k=0.11$. The size of the particles $(5 \mu \mathrm{m})$ in the present research was chosen in order to validate the simulated particle deposition results with the experimental data of Burwash et al. (2006), who used identical particle size of $5 \mu \mathrm{m}$. Moreover, although not reported in the thesis, other particle sizes of 10 and $20 \mu \mathrm{m}$ were used to investigate the effect of their size (Stokes number) on their trajectories.

In the particle modeling in the CFX tracking code, the particles were released during one iteration. Initial particle velocities were set equal to the fluid velocity. The particles were randomly and uniformly distributed in space at the inlet.

In RANS-based particle simulation two types of particle tracking were tested namely "turbulent tracking" and "mean flow tracking". In the turbulent tracking, the 
instantaneous velocity of the flow in the particle equation of motion is equal to the sum of the mean part and the fluctuation part (RMS fluctuating velocity multiplied by a random number). The fluid RMS fluctuating velocities are used without near-wall correction, i.e., as they are obtained from RANS calculations. In the mean flow tracking the instantaneous velocity of the flow is equal to the mean part and the fluctuation part is assumed to be zero.

Additional two computational domains for impinging jet flow with nozzle-tosurface distances of 2 and 6 (i.e., $L / D=2$ and 6 ) were constructed. The dimensions of the computational domains were based on the experiment of Cooper et al. (1993). Indeed, the flow parameters, i.e., the inlet velocity and the Reynolds numbers, were made the same as in their experiment. Figure 4.3 depicts a scheme of the computational domain with its dimensions based on the experiment of Cooper et al. (1993). The height $L$ varies as 52 and $156 \mathrm{~mm}$ leading to nozzle-to-surface distance cases of $L / D=2$ and 6 , where $D=26$ $\mathrm{mm}$ is the nozzle diameter. The purpose of creating these computational domains based on the experiment of Cooper et al. (1993) was to perform fluid flow simulations and thus calibrate the near-wall correction function (i.e., no deposition prediction was performed), which will be used to predict the particle deposition, based on the impinging jet flow characteristics. This will be described later in this chapter.

Before describing the mesh used in the computational domains, it is useful to present details about the experiment of Cooper et al. (1993). In the experimental configuration of Cooper et al. (1993), the flow exits from a pipe and impinges normally on an impingement wall as shown in Figure 4.3, where the side walls and nozzle plate are absent (i.e., unconfined configuration). The authors used a copper pipe with $26 \mathrm{~mm}$ in 
diameter and length of $2100 \mathrm{~mm}$ giving a length to diameter ratio of 80 to 1 . The pipe was long enough to have fully developed flow properties at the exit of the pipe. The end portion of the pipe was slightly inserted inside the domain for stability reasons. Although they used another pipe configuration (i.e., pipe with larger diameter of $101.6 \mathrm{~mm}$ ), they reported the measurement results obtained with the $26 \mathrm{~mm}$ pipe. The impinging jet plate (impingement wall) was made from printed-circuit board secured to thick plywood backing. The circuit board is covered by copper film facing impinging flow and hence enabled the probe's contact with the wall to be identified electrically. On the impinging jet plate there were three probe access holes that were bored to allow profiles to be obtained at the desired radii. The measurements in the impinging jet were conducted with hot-wire anemometer interfaced to a data acquisition system and the values are stored in the computer. In their measurements, in addition to the standard hot-wire probes they used cross-wire probes.

In the experimental procedure, the hot-wire probes were calibrated based on ten equi-spaced velocity increments covering the required range. Several validation checks were made by noting the agreement of the individual data points with a least squares fitting. The cross-wire probes were calibrated in yaw and this calibration was used, as a check, to find out the angle at which the probe was aligned. The maximum error was about $\pm 0.3^{\circ}$. In the measurements, two velocity profile traverses are placed always between probe calibrations. The "before" and "after" calibrations were compared, and the data of the velocity profiles are considered only if the average difference in the calibrated velocities is less than $2 \%$. Although the authors investigated different nozzle-to-surface distance cases, their focus was on $L / D=2$ and 6 . They reported extensive measurements 
including the mean velocity and the RMS velocity fluctuations for $L / D=2$ with $\operatorname{Re}=$ 23000 and $L / D=6$ with $\operatorname{Re}=70000$. The maximum mean velocity at any position has an estimated uncertainty of $\pm 2 \%$. The RMS fluctuating velocities have an estimated uncertainty of $\pm 4 \%$ for the parallel-to-wall component and $\pm 6 \%$ for the normal-to-wall component (Cooper et al., 1993).

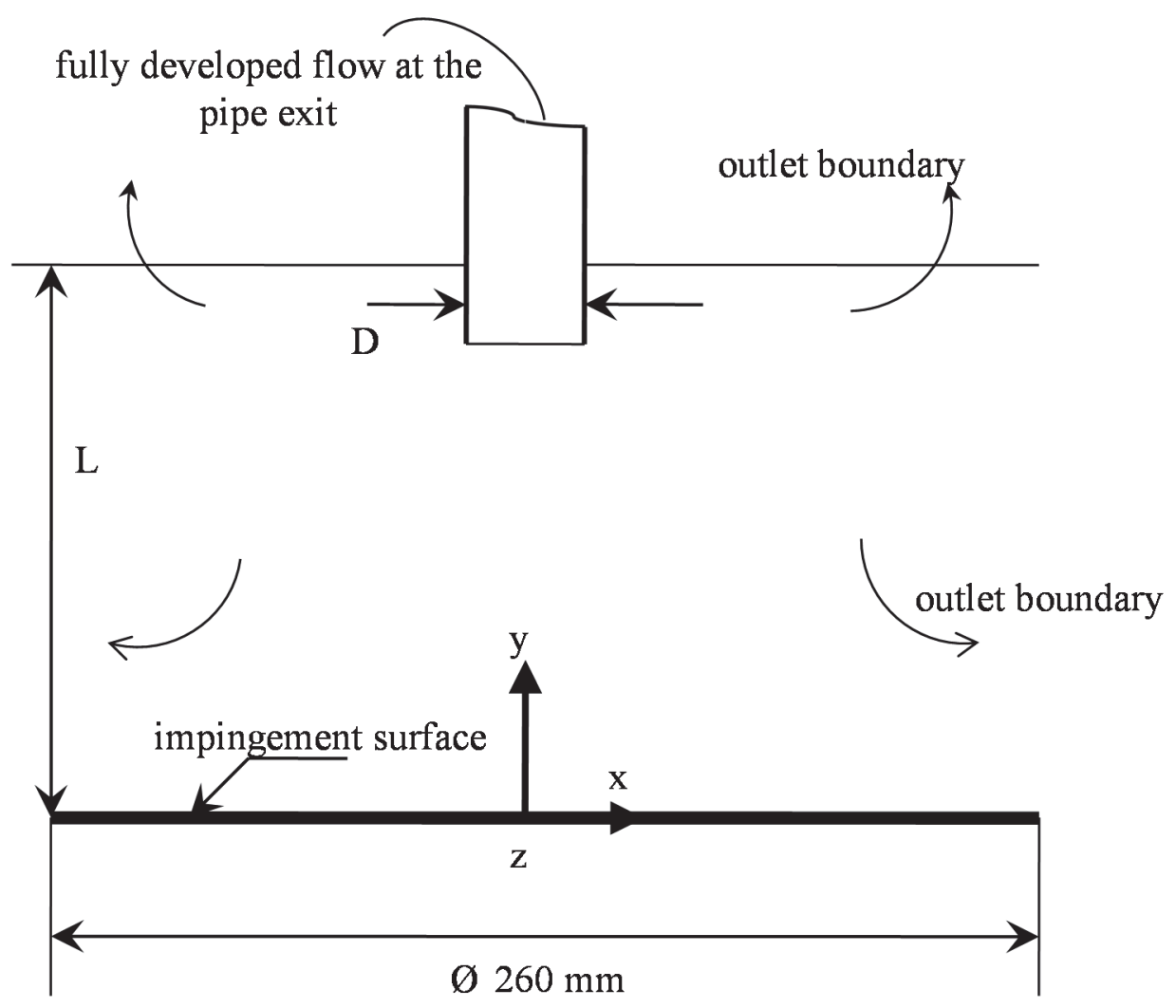

Figure 4.3. Schematic of the computational domain of the impinging jet based on the experiment of Cooper et al. (1993).

The computational domains for the $L / D=2$ and 6 cases were constructed using CFD-ICEM (Ansys Inc.) mesh generator code. Each mesh of these cases is composed of structured multi-block mesh. Each mesh consists of 23 blocks. For each case, the grid po- 


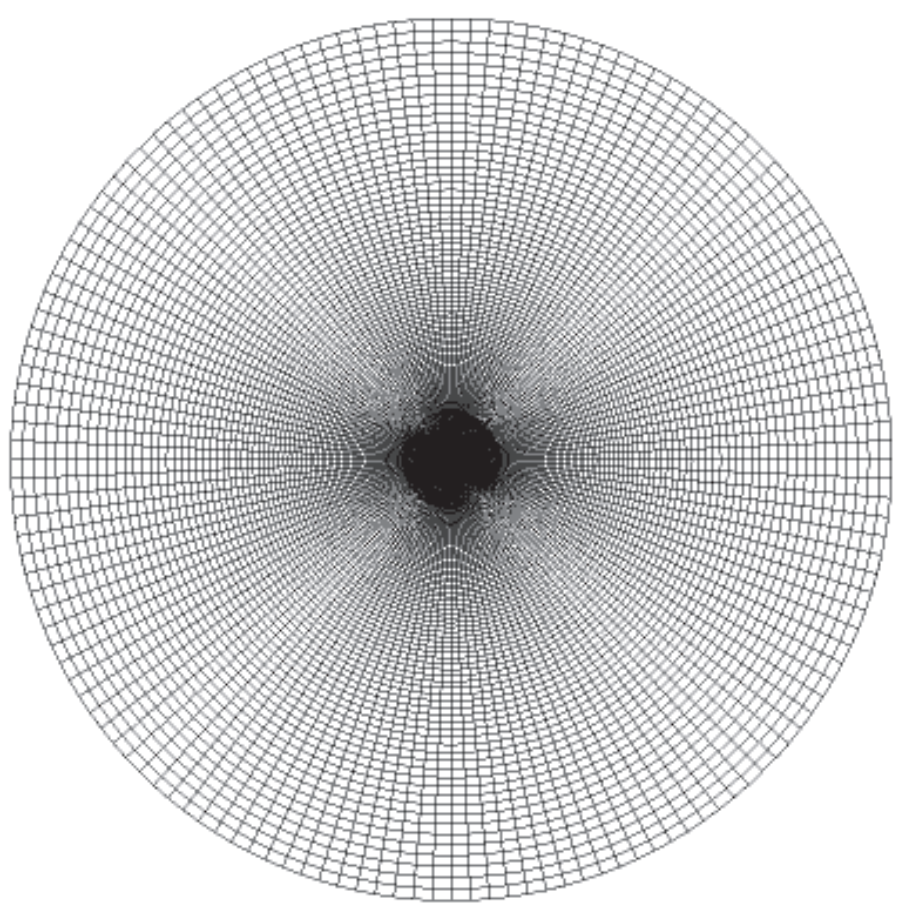

a) Top view of the computational domain.

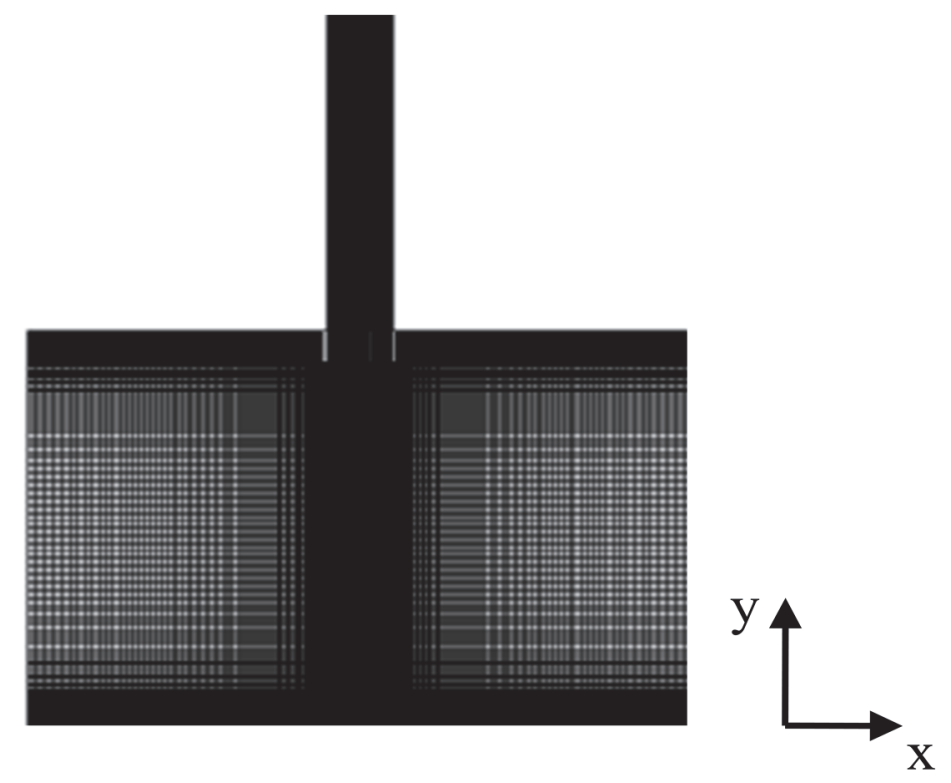

b) Zoom of the normal-to-wall mesh in $x-y$ plane.

Figure 4.4. Grid of the computational domain of the impinging jet used for RANS calculations based on Cooper et al.'s experiment for: (a) Top view of the computational domain, and (b) Zoom of the normal-to-wall mesh in $x-y$ plane. 
ints were accumulated at the bottom, at the top (near the nozzle exit), and in the shear layer of the jet since these are the regions of high velocity gradients. Also, the grid points were clustered in the central region of the geometry (from the inlet up to the stagnation point) and towards the impingement wall. Figure 4.4a shows a top view of the computational domain where the grid points were accumulated in the central region of the domain and Figure 4.4b shows a vertical x-y plane, where a cluster of grid points can be identified close to the impingement wall. As stated before, refining the near-wall region is important when the near-wall correction functions are used to correct the normal-to-wall velocity fluctuations. Thus, the near-wall region was refined such that the values of $y+$ at the first grid point over the impingement wall were in range of about $0.2 \leq$ $y^{+} \leq 1$. Discontinuities in node spacing at the boundaries were avoided in order to have smooth connections. Similar to the case of the computational domains that were based on the experiment of Burwash et al. (2006), a grid convergence analysis was performed using the three grid sizes of $1,310,784,2,422,679$ and $4,537,168$ nodes for $L / D=2$. Based on the grid size that was chosen for $L / D=2$, the grid for $L / D=6$ was established. As described before, this way was adopted based on previous numerical simulations for impinging jet flow (Craft et al., 1993).

For $L / D=6$, the nodes along the $x$ and $z$ axes were kept the same as the chosen grid for $L / D=2$. However, along the normal-to-wall (y-axis) direction, the number of nodes was increased due to the increase in the nozzle-to-surface distance as shown in Table 4.2. Also, the grid density was increased near the wall and reduced gradually in the outward direction with a grid expansion factor of 1.01 for $L / D=2$ and 6 as depicted in Table 4.2. 
Table 4.2. Details of computational domains based on Cooper's experiment.

\begin{tabular}{|c|c|c|c|}
\hline$L / D$ case & $\begin{array}{c}\text { Number of nodes along } \\
\text { the normal-to-wall } \\
\text { direction }\end{array}$ & $\begin{array}{c}\text { Expansion } \\
\text { factor }\end{array}$ & Total number of nodes \\
\hline 2 & 76 & 1.01 & $2,422,679$ \\
\hline 6 & 229 & 1.01 & $4,907,119$ \\
\hline
\end{tabular}

For the inflow boundary, fully developed pipe flow velocity conditions were obtained at the pipe exit. The Reynolds number based on the inlet diameter $(D=26 \mathrm{~mm})$ is $\operatorname{Re}=\rho U_{B} D / \mu=23000$ for $L / D=2$ and $\operatorname{Re}=\rho U_{B} D / \mu=70000$ for $L / D=6$. The bulk velocity is $U_{B}=13.2 \mathrm{~m} / \mathrm{s}$ and $40.1 \mathrm{~m} / \mathrm{s}$, respectively, for $L / D=2$ and 6 . The standard no slip boundary condition was applied at the impingement wall and a constant zero gage pressure opening boundary was applied at the outlet of the computational domain.

In the present research, since there are no researches with complete set of measurements for the fluid flow and the deposition of the particles, two different experimental researches are used in the validation procedure. These are the experiment of Burwash et al. (2006) and the experiment of Cooper et al, (1993). The simulated deposition results (from the CFX and the tracking code) are validated with the experiment of Burwash et al. (2006). However, since the fluid flow results were not reported in the experiment of Burwash et al. (2006), the experiment of Cooper et al. (1993) is used to validate the fluid flow results, which are simulated based on the experiment of Burwash et al. (2006). Therefore, due to the different conditions and configurations between the two experiments, some differences between the present 
simulation and the experiment of Cooper et al. (1993) are expected. This will be described in details in Sections 4.5 and 5.2.

\subsection{Grid convergence analysis}

A grid convergence analysis was performed using RANS with SST model for the $L / D=$ 2 case by considering four different grid sizes $248,676,497,408,761,978$ and 1,007,073. The grid sizes for the $L / D=4$ and 6 cases were constructed based on the adequate grid size chosen for $L / D=2$. The grid convergence analysis was conducted for both the fluid flow and the particle deposition (total particle deposition).

\subsubsection{Fluid Flow}

For the fluid flow, the velocity distribution and the turbulence kinetic energy along the jet centerline axis and along a horizontal line $(z=0.1 D)$ parallel to the impingement surface were used to test the grid convergence for the flow field. The following two subsections describe in details the grid convergence analysis for the fluid flow.

\subsubsection{Velocity distribution}

Figure 4.5 shows the velocity magnitude along the centerline axis as a function of $z / D$.

From the figure, as the airflow exits the nozzle, the potential core is formed and the velocity remains approximately equal to the jet exit velocity. The potential core is formed due to turbulent mixing similarly as the free jet flow (Ashforth-Frost et al., 1997). However, once the shear layer grows and penetrates the jet axis, the potential core ends and the velocity starts to decay before it reaches zero at the impingement wall. The figure indicates that the velocities for the four grid sizes decay with the same trend as the flow approaches the impingement wall. 


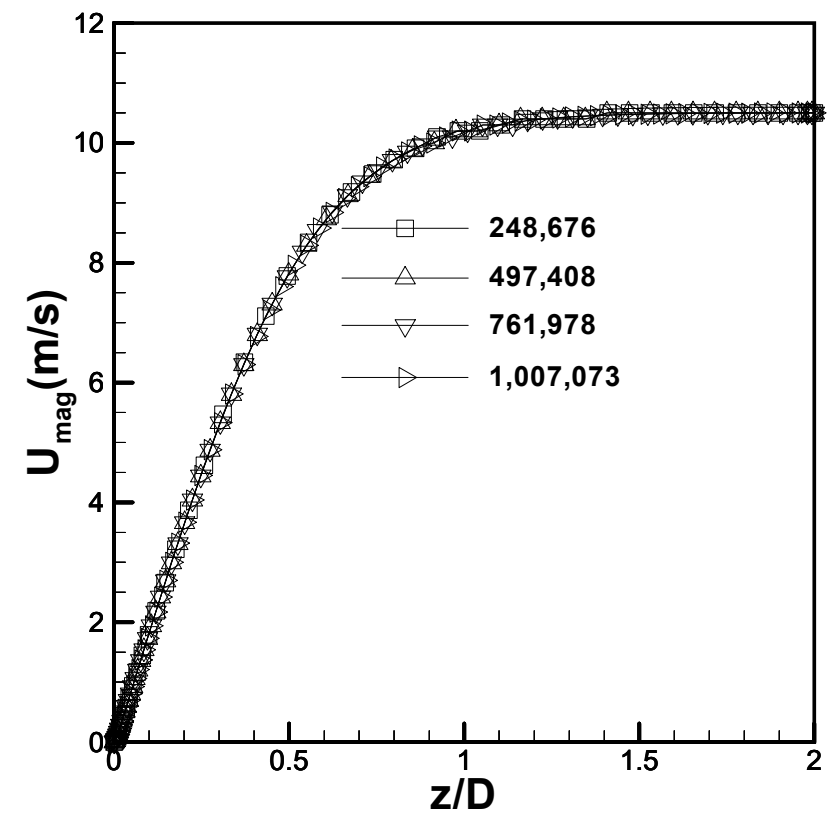

Figure 4.5. Velocity magnitude along the centerline axis.

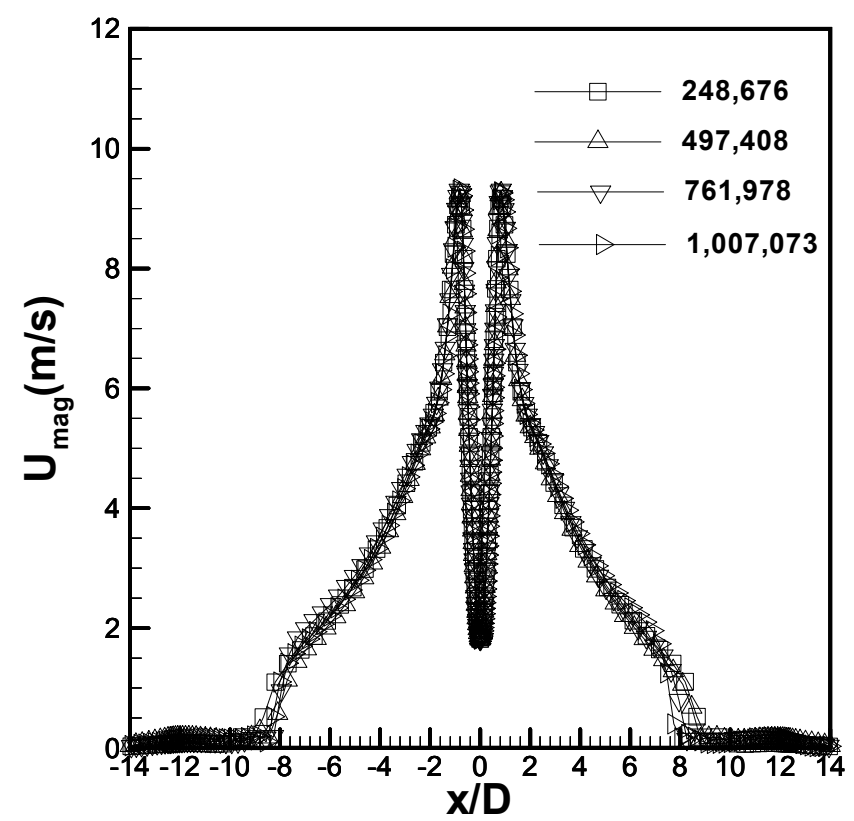

Figure 4.6. Velocity magnitude along a line parallel to the impingement wall $(\mathrm{z}=$ 0.1D). 
Figure 4.6 shows the velocity along a line parallel to the impingement wall $(z=$ $0.1 D$ ) as a function of $x / D$. This figure shows that the velocity profiles for the four grid sizes collapse on each other showing a symmetric behavior around the centerline axis. The velocity along the centerline axis (stagnation line) is decaying as shown at the center $(x / D=0)$. However, in the wall jet region ( just on the sides of the stagnation region) the velocity is increasing to two symmetric peaks, due to the turbulent shear flow produced in the wall jet region, before it vanishes in the far field.

\subsubsection{Kinetic energy}

Figure 4.7 shows the kinetic energy along the centerline axis as a function of $z / D$. As can be seen from the figure, the kinetic energy for the four grid sizes is decaying gradually before the flow reaches the impingement wall. Then, it increases due to the normal straining before decaying sharply at the wall.

Figure 4.8 illustrates the kinetic energy profiles along a line $(z=0.1 D)$ parallel to the impingement wall versus $x / D$. It can be noted that the kinetic energy profiles for the four grids are identical to each other and symmetric around the centerline axis. As can be noted from the figure, higher kinetic energy peaks result on both sides of the wall jet, in a direction parallel to the impingement wall, due to the turbulent shear produced. The energy production is reduced gradually as the distance is increased away from the stagnation point, which implies that the wall jet vanishes.

From the grid convergence analysis, it can be seen that a number of 761,978 nodes is sufficient for accurate results. 


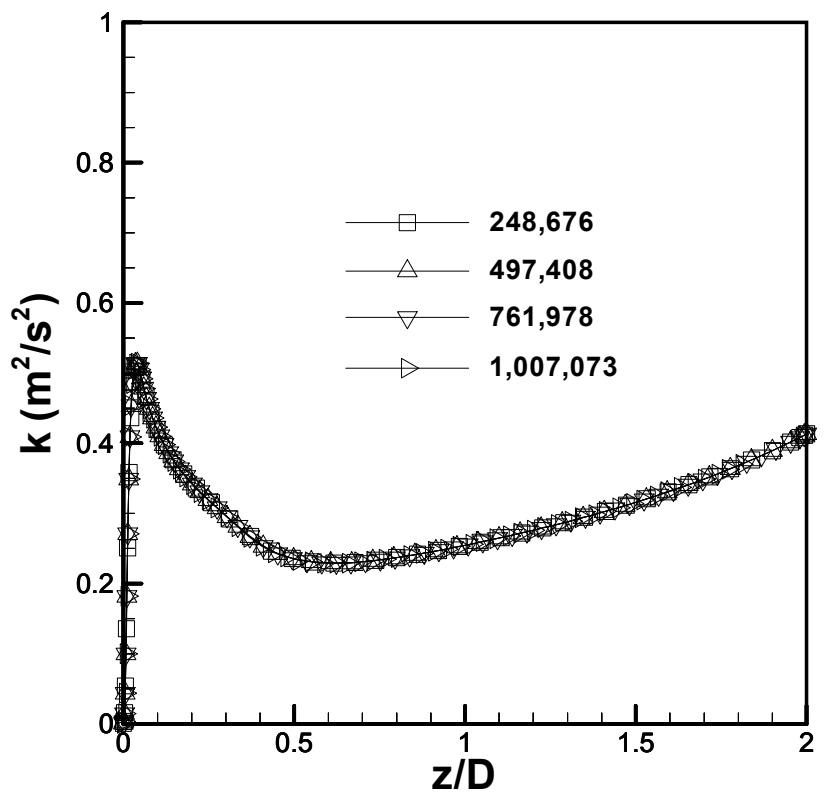

Figure 4.7. Kinetic energy along the centerline axis.

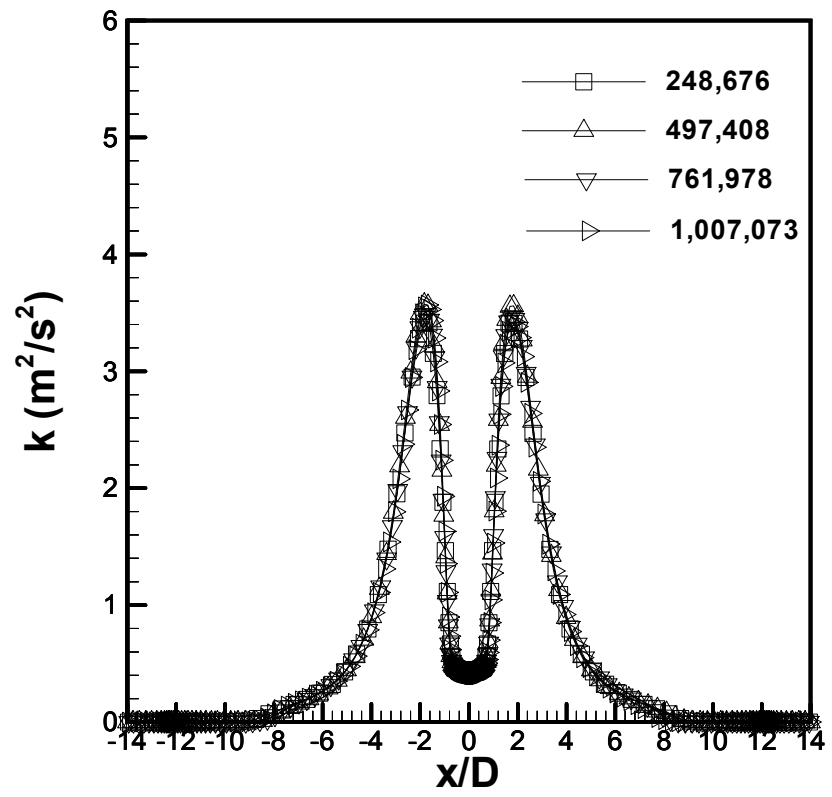

Figure 4.8. Kinetic energy profiles along a line parallel to the impingement surface $(\mathrm{z}=0.1 \mathrm{D})$. 


\subsubsection{Grid effect on particle deposition}

Figure 4.9 shows the influence of the grid resolution on the particle deposition results for the four grid sizes. In this calculation, the turbulent tracking was used. As shown in the figure, the particle deposition is almost constant as the grid size is increased. The particle deposition is showing nearly the same value of deposition percentage of approximately $11 \%$. This indicates the adequacy of the grid resolution and, therefore, grid size of 761 , 978 nodes was used for accurate deposition prediction.

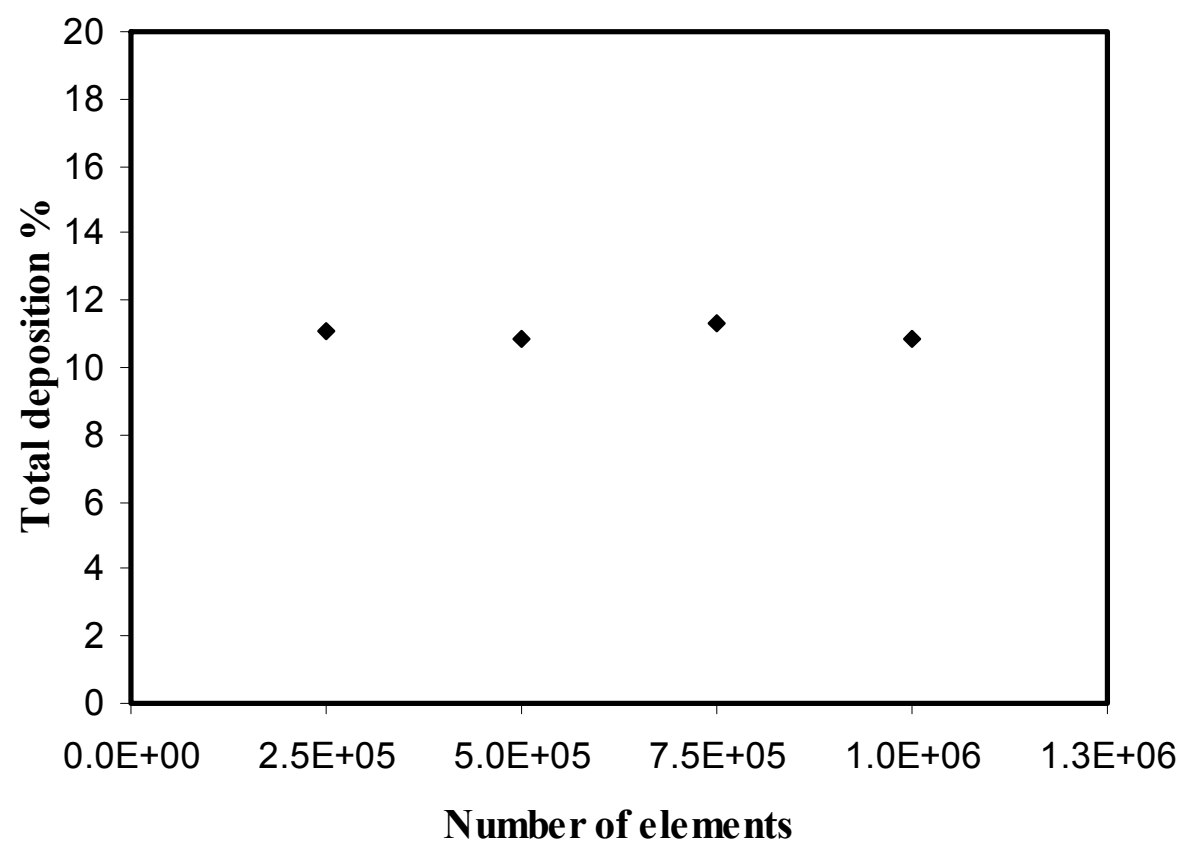

Figure 4.9. Effect of grid resolution on the particle deposition.

\subsection{Effect of the number of particles}

The effect of the number of particles on the deposition is investigated using six different values, $N_{p}=1000,2000,5000,7000,10,000$ and 20,000. Figure 4.10 illustrates the 
relative variation of the total percentage of deposition versus the number of particles released in the computational domain. As can be shown from the figure, the relative variation is very small for number of particles less than 10,000 beyond which this variation becomes insensitive as the number of particles is increased to 20,000 particles. Since the deposition using 10,000 particles shows no difference when compared with the deposition using 20,000 particles, this means that 10,000 particles is adequate for deposition statistics. Therefore, $N_{p}=10,000$ particles is used in the present research.

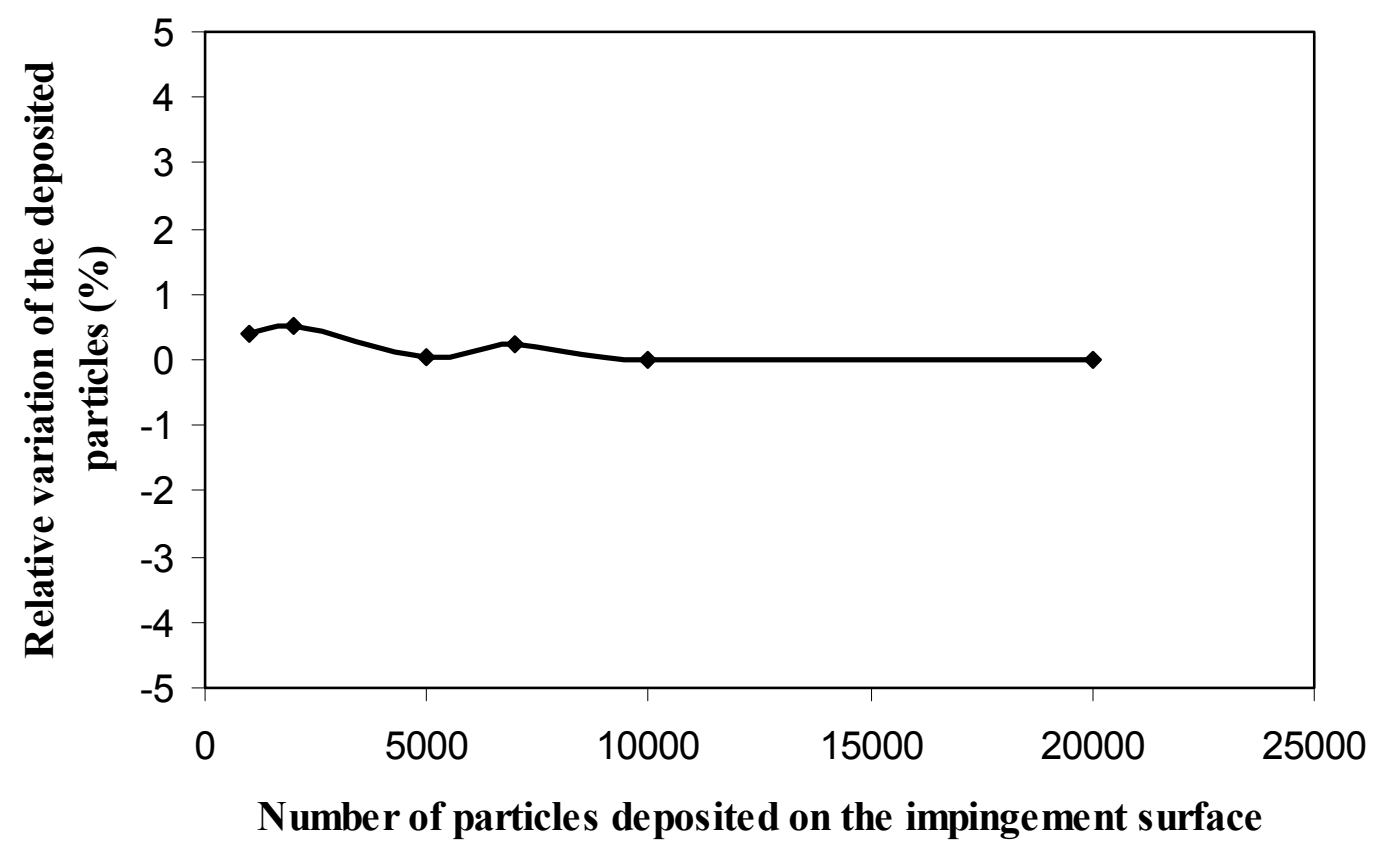

Figure 4.10. Effect of number of particles on the deposition.

\subsection{Sensitivity analysis for the minimum Lagrangian time scale}

As was mentioned previously, the minimum value of the Lagrangian time scale, $\tau_{L}$, was used in the tracking code in order to avoid the problem that occurs near the wall, where 
eddy lifetimes reach to infinitesimal values. The effect of the minimum Lagrangian time scale on the prediction of particle deposition density is investigated by decreasing the Lagrangian time scale from $1 \times 10^{-4}$ to $1 \times 10^{-7} \mathrm{~s}$. Figure 4.11 shows the deposition density for $L / D=2$ plotted as a function of $r / D$ using four values of the minimum Lagrangian time scale. The figure shows that the deposition density changes substantially when $\tau_{L}$ is reduced from $1 \times 10^{-4}$ to $1 \times 10^{-5} \mathrm{~s}$. However, the changes in the deposition density becomes nearly insensitive as $\tau_{L}$ is reduced further from $\tau_{L}=1 \times 10^{-5}$ to $1 \times 10^{-7} \mathrm{~s}$. For these reasons, and because of computational time savings, $\tau_{L}=1 \times 10^{-5} \mathrm{~s}$ is chosen in the present study as the minimum Lagrangian time scale.

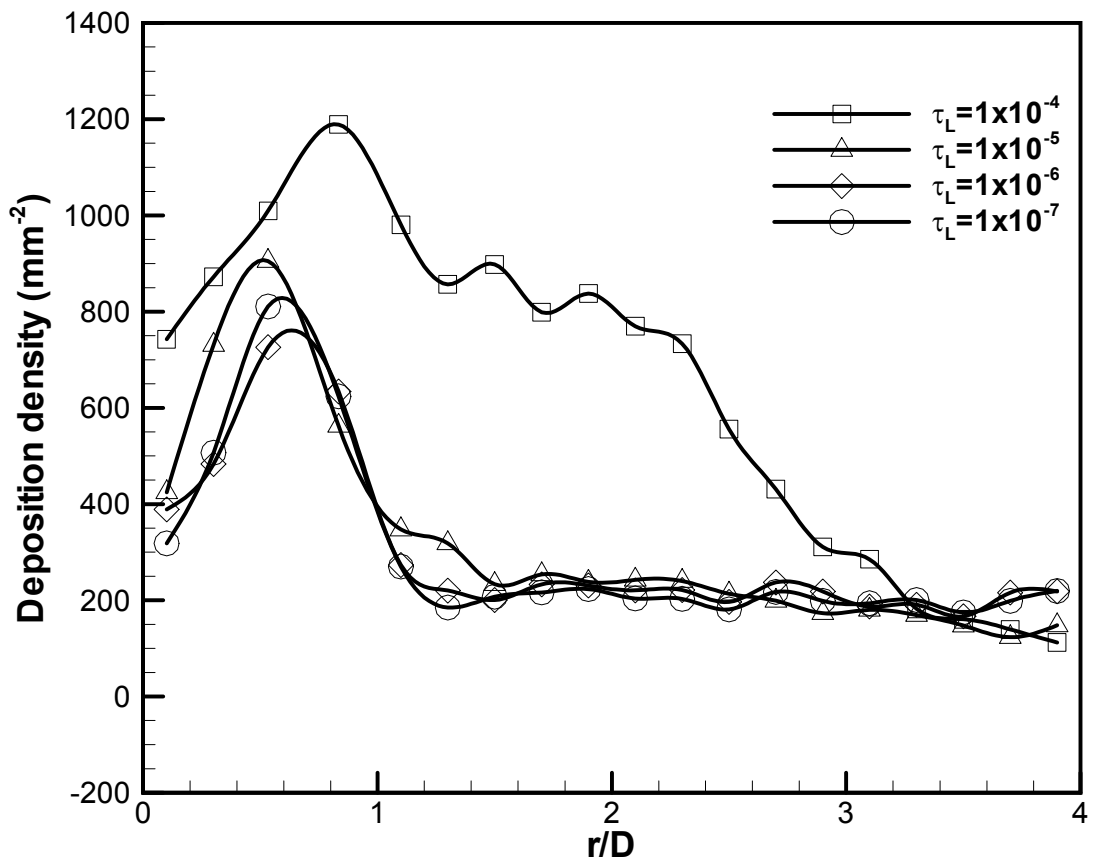

Figure 4.11. Sensitivity analysis for the minimum Lagrangian time scale. 


\subsection{Fluid Flow}

The fluid flow has strong influence on the particulate phase. Therefore, it is important to investigate the impinging jet flow field before predicting the deposition. The profiles of the mean velocity magnitude and the root-mean-square (RMS) normal-to-wall fluctuating (computed as $(2 / 3 k)^{0.5}$, i.e., $\left.w_{f r m s}^{\prime}=(2 k / 3)^{0.5}\right)$ velocities along lines perpendicular to the impingement wall versus the dimensionless distance from the wall $z / D$ for $L / D=2$ are shown in Figures 4.12a-4.12c and 4.12d-4.12f, respectively. The mean velocity magnitude and the RMS fluctuating velocities are normalized with the nozzle exit velocity $U_{j}$. The simulated mean magnitude and RMS fluctuating velocities (lines with open square symbols) are compared with the experimental data of Cooper et al. (1993) (dark square symbols). The experimental data of Cooper et al. (1993) are shown here as a reference since the present simulation, which is based on the experiment of Burwash et al. (2006), has different flow parameters and conditions from the experiment of Cooper et al. (1993). As can be noted in Figures 4.12a-4.12c, the simulated magnitude profiles of the mean velocity have similar trends as the experiment. Also, it can be noted that although the simulated mean profiles at $x / D=0.5$ and 1 are underestimated when compared to the experimental peaks close to the impingement wall, the profile at $x / D=$ 2.5 is slightly overestimated. However, they have a similar decay rate as the experiment in the viscous sublayer region (i.e., at $z / D \leq 0.01$ ). Moreover, the spreading of the mean velocity into the freestream is qualitatively similar to the experiment. The differences between the present simulation and the experimental data are described in the coming paragraphs. 
a)

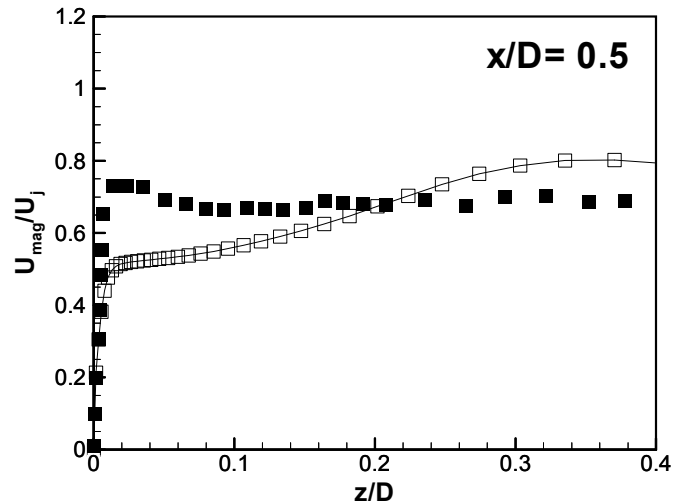

b)

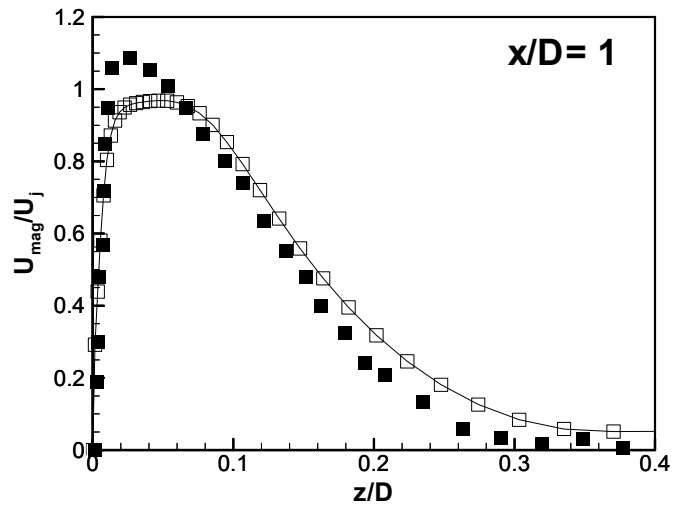

c)

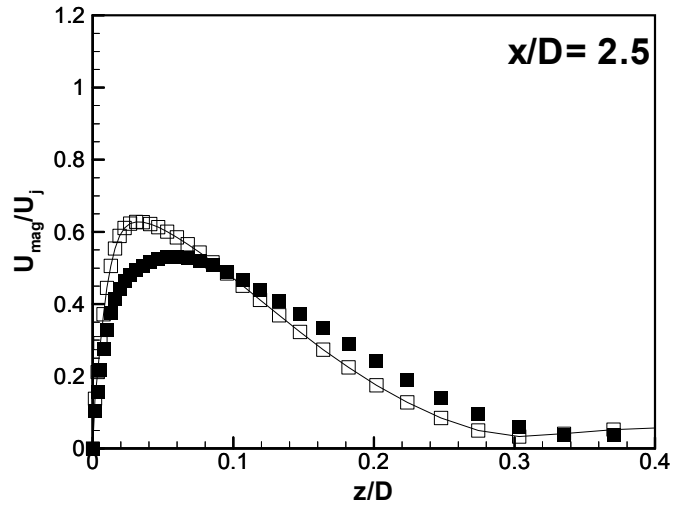

d)

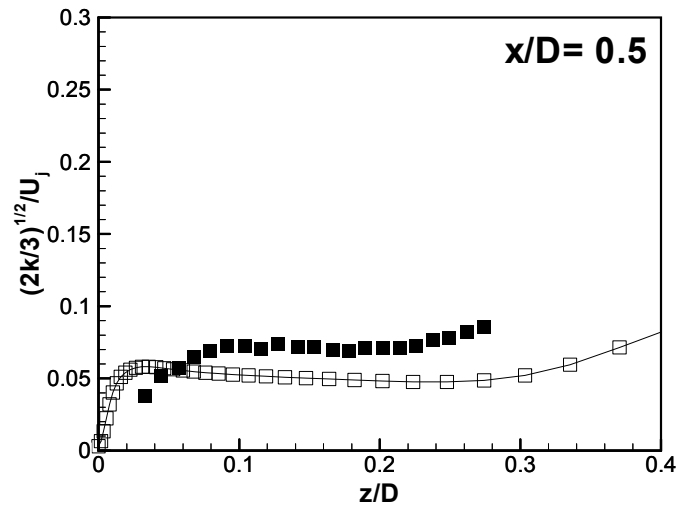

e)

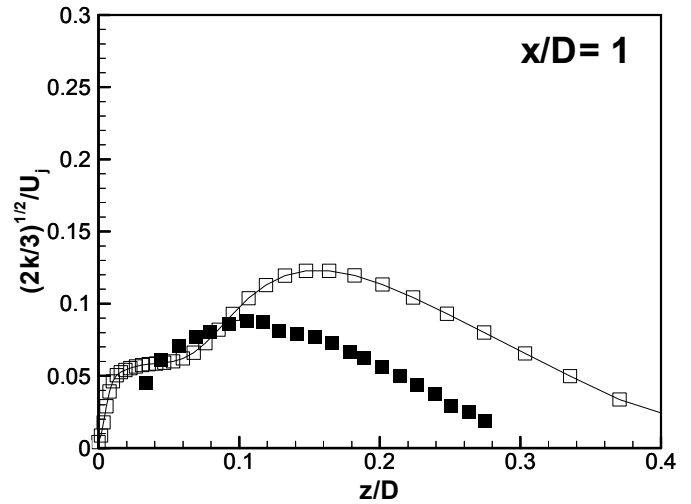

f)

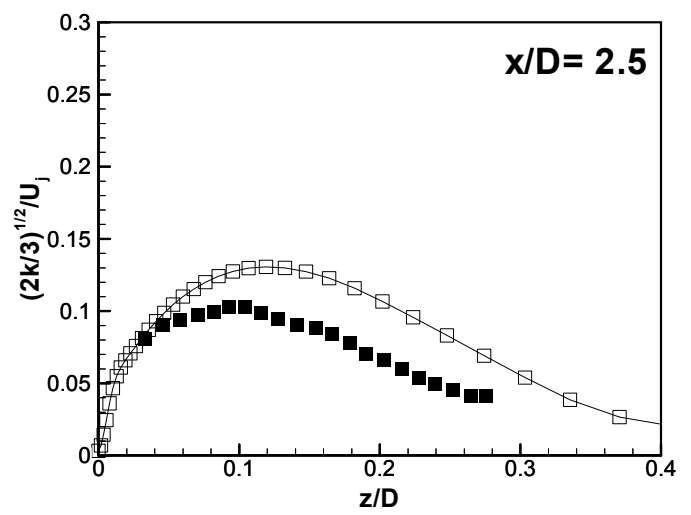

Figure 4.12. Mean velocity magnitude (a-c), and normal-to-wall fluctuation (d-f) for $\mathrm{L} / \mathrm{D}=2$ using RANS SST model at three stations: $\mathrm{x} / \mathrm{D}=\mathbf{0 . 5}, 1$ and 2.5 . 
The RMS fluctuating velocities (Figure 4.12d-4.12f), in general, have similar trends as the experiment; however, they are overestimated when compared to the experiment as the impingement wall is approached (i.e., at $z / D \leq 0.04$ ). Except for the fluctuating velocity at $x / D=0.5$, the simulated fluctuations are overestimated in comparison to the experiment over most of the dimensionless distance $z / D$.

Similarly for $L / D=6$, the profiles of the mean magnitude and the RMS fluctuating velocities along lines perpendicular to the impingement wall versus $z / D$ are shown in Figures 4.13a-4.13c and 4.13d-4.13f, respectively. The experimental data of Cooper et al. (1993) (dark square symbols) are also shown here as a reference. As can be seen from Figures $4.13 \mathrm{a}-4.13 \mathrm{c}$, the simulated mean profiles have similar trends as the experiment, although they are underestimated slightly when compared to the experimental peaks near the impingement wall. Also, they have similar decay rate as the experiment very close to the wall. With the exception of the fluctuating velocity at $x / D=0.5$, which has a prediction that is close to the experiment, the fluctuations at $x / D=1.5$ and 3 demonstrate higher predictions than the experiment further away from the wall, even though the fluctuation at $x / D=3$ is reduced to the level of the experimental fluctuation at about $z / D$ $<0.1$ (Figures 4.13d-4.13f)

The mean velocity trends (in the experiment and simulation for $L / D=2$ ) at $x / D=$ 0.5 (Figure 4.12a) are slightly different from the two downstream trends at $x / D=1$ and 2.5 (Figure $4.12 \mathrm{~b}$ and $\mathrm{c}$ ). This because at $x / D=0.5$ the mean velocity is still affected by the pressure close to the stagnation point unlike the trends at $x / D=1$ and 2.5 where the RMS fluctuations contribute to their behaviors more than the pressure effect. The mean velocity profiles at $x / D=1$ and 2.5 exhibit higher values close to the wall (demonstrated 
by the peaks just before they come under the wall effect) due to the large shear produced during the wall jet development. As $z / D$ increases, the profiles fall gradually and reach very small values away from the wall as the shear weakens. The behaviors of the RMS fluctuating velocities at $x / D=1$ and 2.5 (Figures $4.12 \mathrm{e}$ and $\mathrm{f}$ ) is related to the mean flow behavior in these regions where the shear produces large turbulence kinetic energy leading to higher fluctuations as seen in their trends (in the experiment and simulation) before they decay due to the wall effect. It can be noted that the RMS fluctuation at $x / D=$ 0.5 (Figure 4.12d) has lower trend than those at $x / D=1$ and 2.5 because the wall jet in this region is at the beginning of the development stage and hence has low turbulence kinetic energy. For $L / D=6$, the behaviors of the mean and RMS fluctuating velocities shown in Figure 4.13 are qualitatively similar to those described for $L / D=2$. However, the higher nozzle-to-surface distance effect $(L / D=6)$ leads to higher RMS fluctuations (as shown in Figures 4.13d-4.13f) than those for $L / D=2$ due to higher kinetic energy produced in the $L / D=6$ case.

The differences between the simulation results and the experiment of Cooper et al. (1993) in Figures 4.12 and 4.13 are expected because of the different flow parameters and inflow conditions used in the simulation and the experiment. In the present simulation, the flow parameters such as Reynolds number are set to values identical to those of the experiment of Burwash et al. (2006), who reported only the deposition results. In the experiment, the Reynolds number is 10000 for all nozzle-to-surface distance $L / D$ cases. However, in the experiment of Cooper et al. (1993), the Reynolds numbers used were 23000 and 70000 for $L / D=2$ and 6 , respectively. Another important reason for these differences is the inflow condition, which is believed to have a significant effect on the 
a)

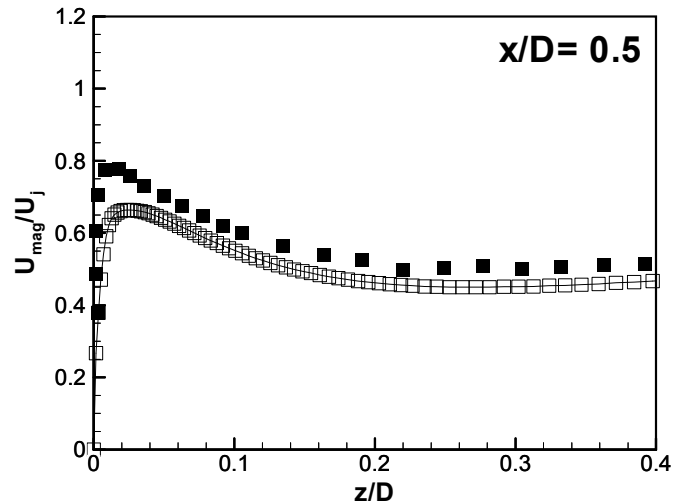

b)

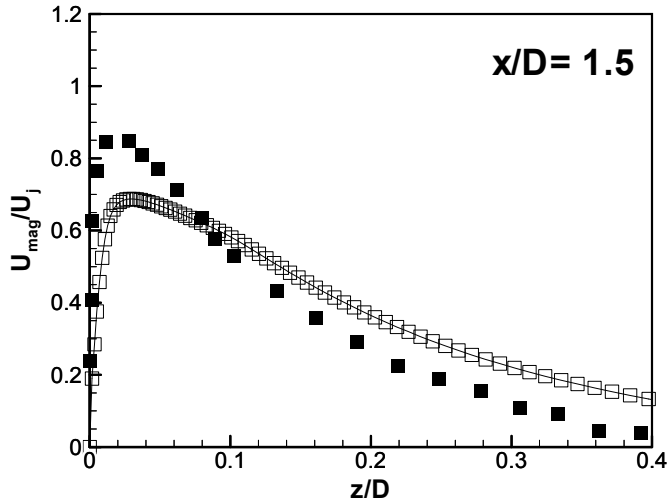

c)

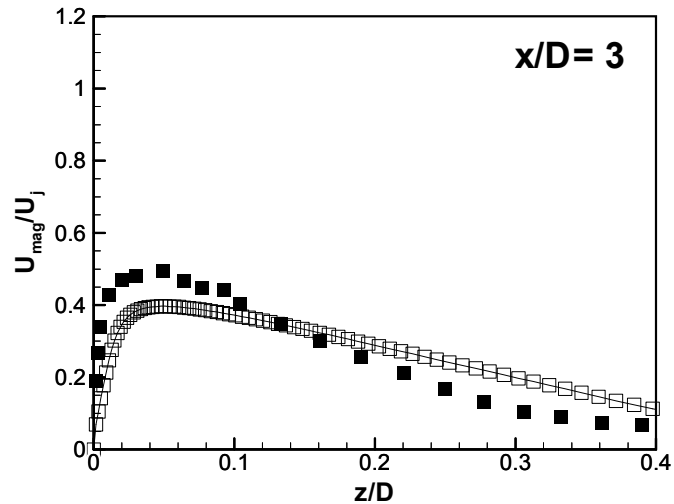

d)

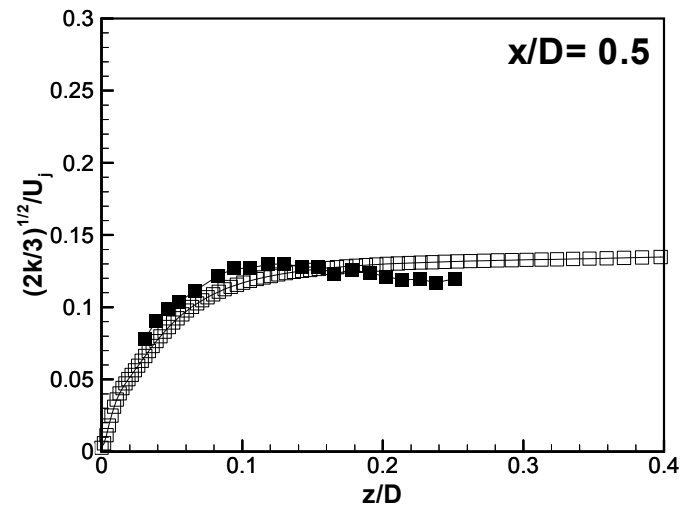

e)

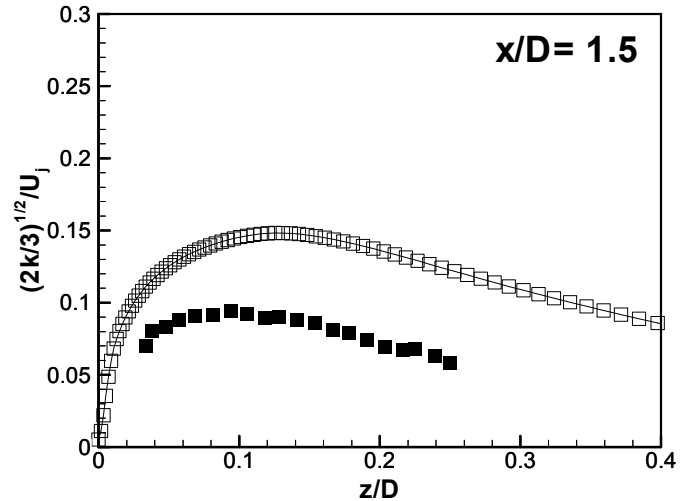

f)

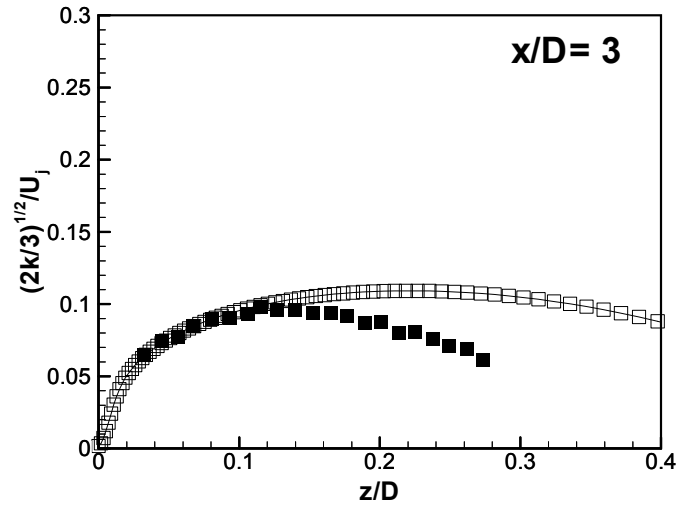

Figure 4.13. Mean velocity magnitude (a-c), and normal-to-wall fluctuation (d-f) for $\mathrm{L} / \mathrm{D}=6$ using RANS SST model at three stations: $\mathrm{x} / \mathrm{D}=0.5,1.5$ and 3 . 
fluid flow. In the present simulation, which is performed under the conditions of the experiment of Burwash et al. (2006), the inflow velocity profile is nearly flat (Top-Hat profile), where the exit was a contraction nozzle. In contrast, in the experiment of Cooper et al. (1993), the flow exits from a pipe nozzle. Finally, the present simulations are conducted in confined computational domains (similar to Burwash et al. (2006) experimental set-up) unlike the experiment of Cooper et al. (1993), which has an unconfined set-up.

The overprediction in the normal-to-wall fluctuating component, which is shown for $L / D=2$ and 6 (Figures 4.12 and 4.13), can be attributed to the excessive prediction of the turbulence kinetic energy in the wall jet and stagnation regions as obtained from the SST model although the stress limiter employed in the model. In addition, the SST model is insensitive to the streamline curvature effect due to the eddy viscosity assumption used in the model. Also, the variations between the actual inlet conditions in the experiment and those used for the simulation may affect the prediction of the fluctuating velocities, which are more sensitive to these variations, and consequently affect the mean flow prediction. The differences between the simulation and the experiment, which are noted in the mean velocity profiles, are influenced by the corresponding differences seen in the fluctuating components, since the mean flow is linked to the fluctuating components via the energy transfer process.

As shown in Figures 4.12 and 4.13, the RANS SST model is unable to accurately predict the normal-to-wall fluctuating velocity near the impingement wall. The overprediction in the normal-to-wall fluctuating velocity component, which is responsible for the deposition, has consequences in predicting high deposition rates as will be shown 
in Subections 4.6.1 and 4.9.1. Therefore, for successful predictions of the particle deposition rate, it is necessary that the normal-to-wall fluctuating velocity be correctly modeled. This is discussed more in the next sections.

\subsection{Deposition results using classical EIM}

\subsubsection{Turbulent tracking and mean flow tracking}

The particle deposition density profiles, using turbulent tracking (i.e., turbulent dispersion) and mean flow tracking (i.e., without turbulent dispersion) versus the radial distance from the stagnation point, $\mathrm{r} / \mathrm{D}$, are shown in Figures 4.14, 4.15 and 4.16 for $L / D$ $=2,4$ and 6, respectively. These particle simulations were performed using CFX tracking code (classical EIM). The experimental data of Burwash et al. (2006) for $L / D=2,4$ and 6 are shown for comparison. As demonstrated in Figures 4.14, 4.15 and 4.16 that the turbulent tracking (shown as bold solid lines) tends to overpredict the deposition density when compared to the experiment, at $r / D \leq 1.8$ for $L / D=2$ and 4 , and over the entire range of $r / D$ for $L / D=6$ cases. In all the cases, the maximum overprediction of the deposition density takes place approximately in a region in which the wall jet starts to develop where the shear replaces normal straining as the principle energy mechanism that produces the kinetic energy. This overprediction is not unexpected because of the isotropy assumption employed in the SST turbulence model. The isotropy assumption gives higher turbulence intensities (i.e., excessive turbulence kinetic energy) than the actual component normal to the wall, which plays an important role for particle deposition, and thus causes more deposition. From the figures, it can be deduced that the 
accurate modeling of the near-wall effects on turbulence features is important for the prediction of the deposition rates.

In contrast to the turbulent tracking, the mean flow tracking (thin solid lines), where the particles are deposited by the means of inertial impaction mechanism, underpredicts the deposition density, when compared to the experiment, over the entire range of $r / D$ for the $L / D=2$ and 4 cases. However, it has a better prediction for $L / D=6$ in the wall jet region although it causes an underprediction of the deposition density at approximately $r / D<0.6$. The underprediction trend (for $L / D=2,4$ and 6 cases) for the deposition density is expected because at a size of $5 \mu \mathrm{m}$ most of the particles, due to their small inertia, follow the mean flow field and only a few of them deposit on the impingement wall. These results therefore suggest that the turbulent dispersion mechanism which drives the turbulent tracking is the main mechanism that causes the particles to deposit on the impingement wall.

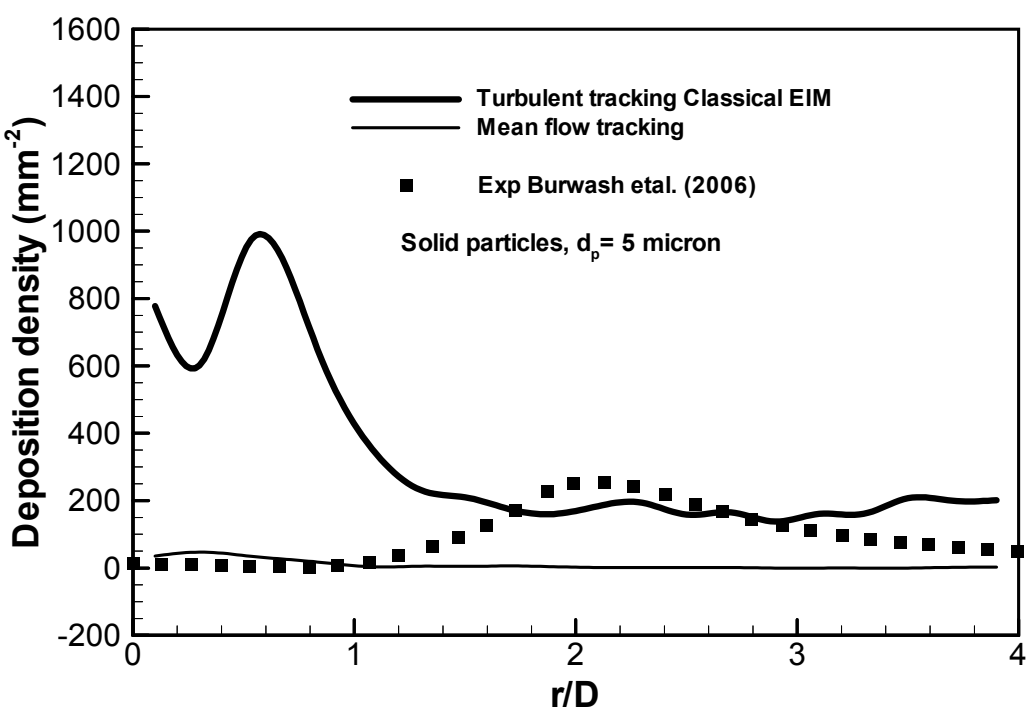

Figure 4.14. Deposition density using turbulent tracking and mean flow tracking for L/D = 2 using classical EIM. 


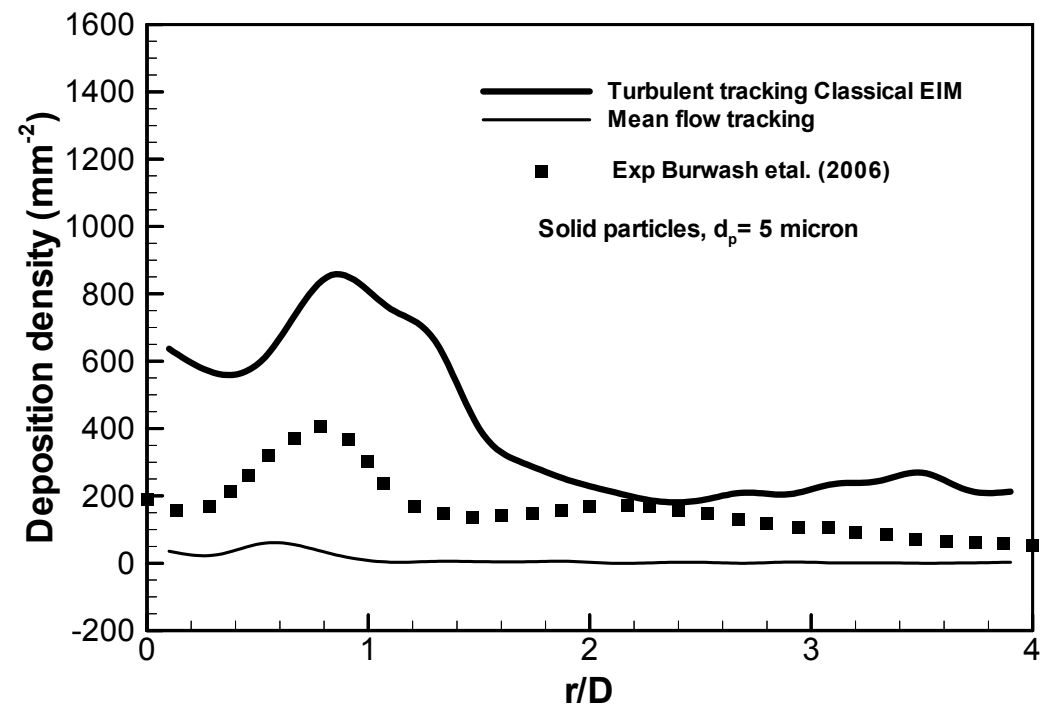

Figure 4.15. Deposition density using turbulent tracking and mean flow tracking for L/D $=4$ using classical EIM.

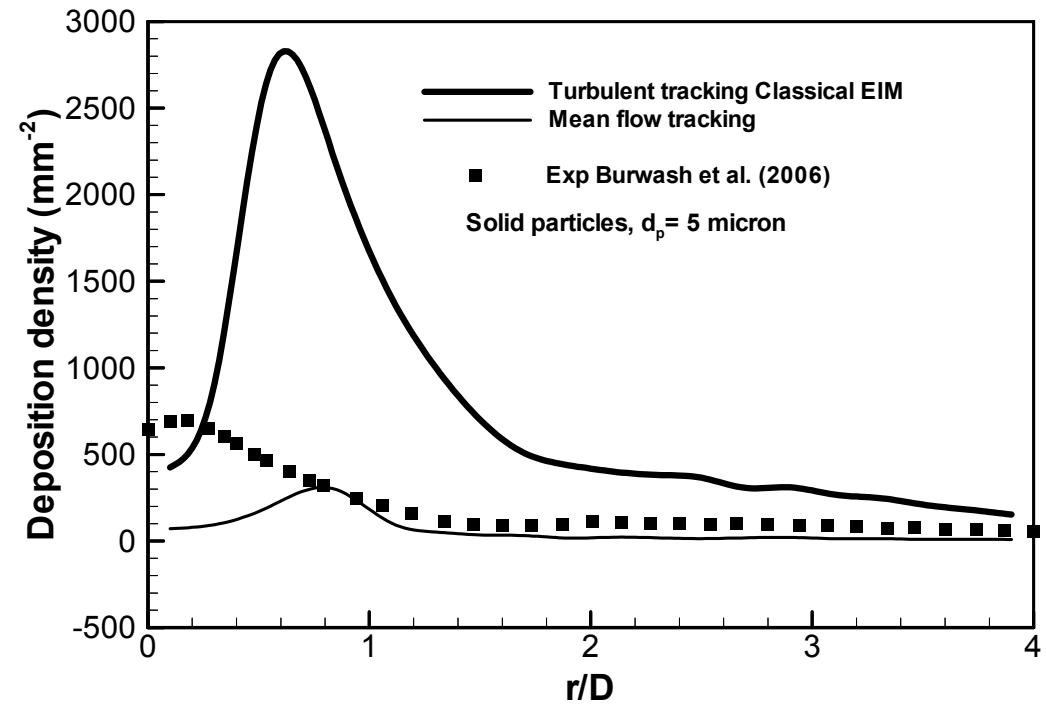

Figure 4.16. Deposition density using turbulent tracking and mean flow tracking for L/D $=6$ using classical EIM. 


\subsubsection{Classical EIM using eddy ratio correction}

In order to correct the particle deposition behavior predicted by turbulent tracking, the eddy ratio correction method is adopted in the present study. The eddy ratio correction is implemented by CFX using classical EIM. In this correction, a hybrid of turbulent tracking and mean flow tracking using different levels of correction by different eddy viscosity ratios was used for a robust prediction of particle tracking. In turbulent tracking, the instantaneous fluid velocity is equal to the sum of the mean fluid velocity and the fluctuating fluid velocity, whereas in mean flow tracking the instantaneous fluid velocity is equal to the mean fluid velocity, i.e., the fluctuation velocities are assumed to be zero. For the hybrid turbulent and mean flow tracking, the local turbulent and laminar regions at the particle location are detected using the ratio between the eddy viscosity and the dynamic fluid viscosity, $v_{\mathrm{T}} / \mathrm{v}$, as defined by

$$
\begin{array}{ll}
v_{T} / v<\gamma & \text { Laminar flow } \\
v_{T} / v>\gamma & \text { Turbulent flow }
\end{array}
$$

where $\gamma$ is a user defined ratio. If the eddy ratio exceeds the user defined ratio (the CFX default ratio is 5), then the flow is considered to be turbulent and turbulent tracking is implemented. On the other hand, if the eddy ratio is below the user defined ratio, the flow is laminar and mean flow tracking is used.

The particle deposition density profiles, with different levels of eddy viscosity ratios (using Equation 4.2), versus $r / D$ are shown in Figures 4.17, 4.18, 4.19 for $L / D=2$, 4 and 6 , respectively. In the case of $L / D=2$, with the exception of eddy ratio of 2.5 , which leads to an overprediction of the deposition density at about $r / D \leq 1.6$, eddy ratios of 7.5 and 10 lead to an underprediction in the deposition density curves when compared 
to the experiment of Burwash et al. (2006) (dark square symbols) at about $r / D \geq 1.2$. However, eddy ratio correction of $5\left(v_{T} / v=5\right.$, dot dash lines) predicts the deposition peak (in magnitude) quite close to the experiment although its location is shifted slightly to the left of the experimental peak at about $r / D \approx 1.1$.

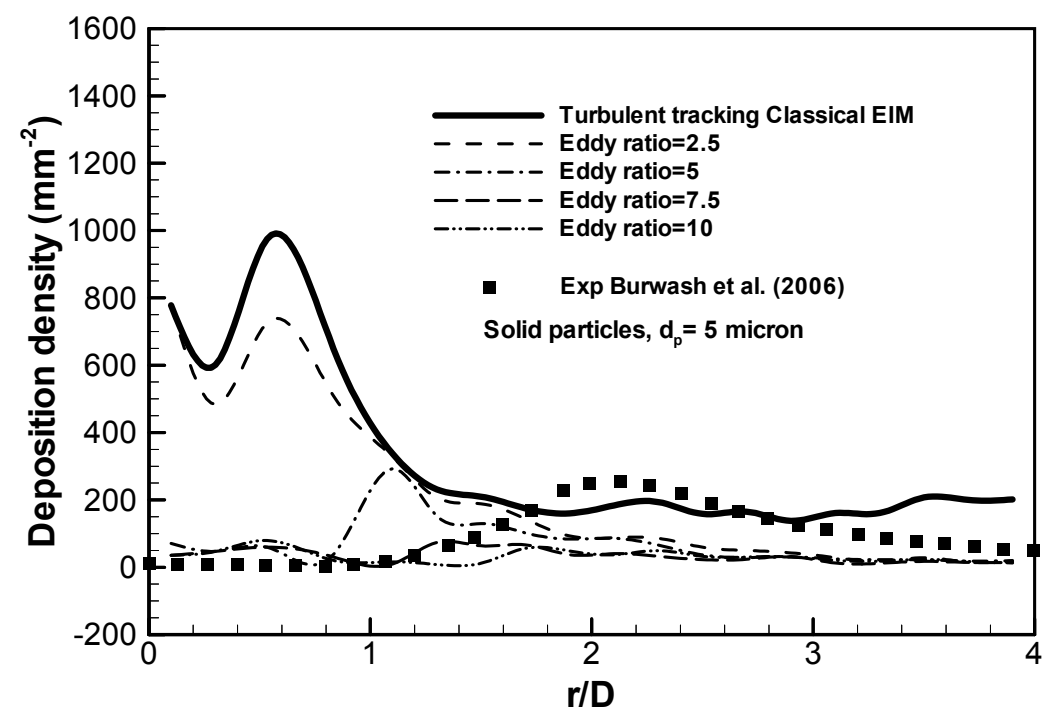

Figure 4.17. Effect of eddy viscosity correction on deposition density for $L / D=2$.

For the case of $L / D=4$ (Figure 4.18), the correction with different eddy ratios leads to several behaviors for the deposition density curves. However, the eddy ratio corrections of $7.5\left(v_{T} / v=7.5\right.$, long dash lines $)$ and $10\left(v_{T} / v=10\right.$, dot dot dash lines $)$ yield deposition density curves that behave closely as the experiment although their deposition peaks occur slightly downstream the experimental peak. At larger nozzle-tosurface distances, $L / D=6$ (Figure 4.19), the deposition density curve is reduced as the eddy ratio is increased from 10 to 50 . The eddy ratio of $50\left(v_{T} / v=50\right.$, dot dot dash 
lines) leads to a prediction that is close to the experimental deposition at $r / D>0.6$ although it underpredicts the experimental peak close to the stagnation point.

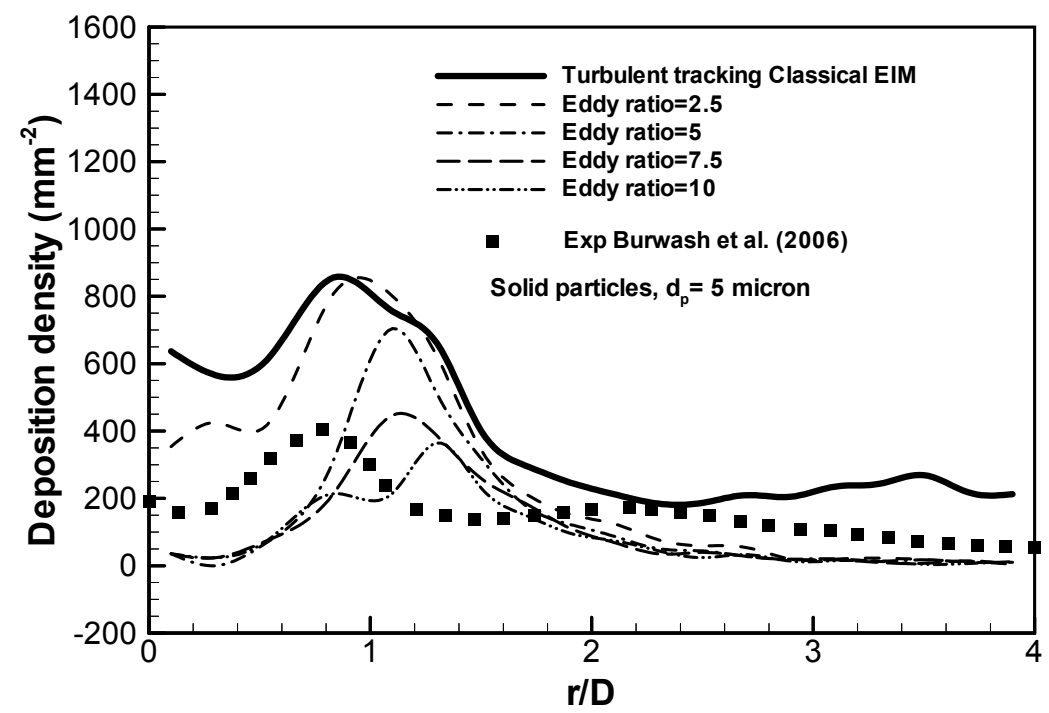

Figure 4.18. Effect of eddy viscosity correction on deposition density for $L / D=4$.

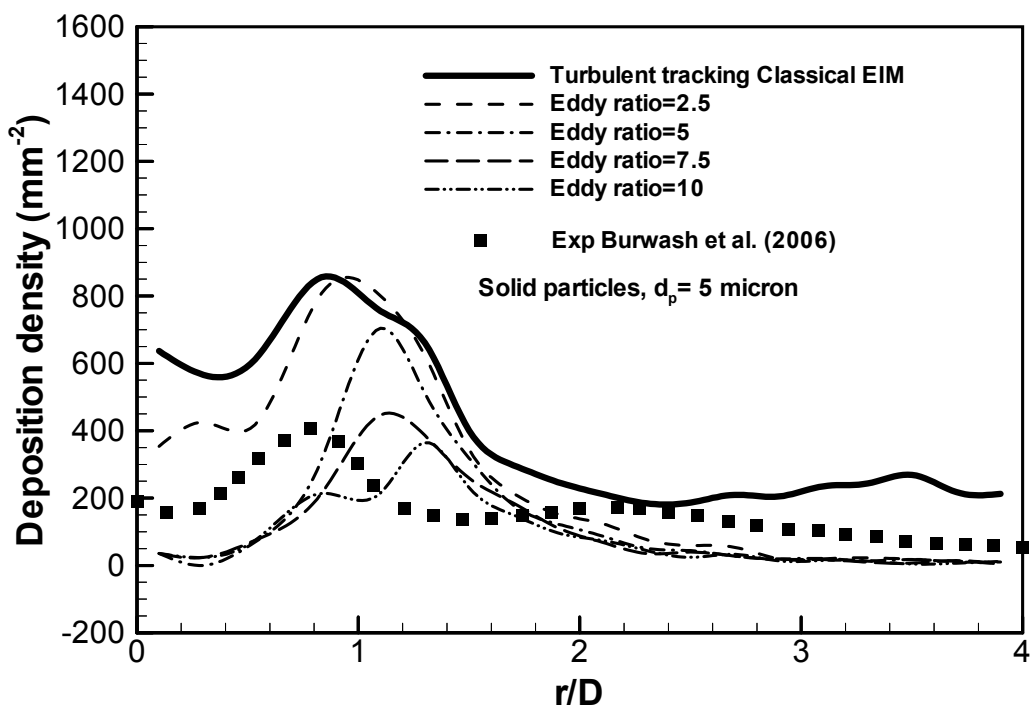

Figure 4.19. Effect of eddy viscosity correction on deposition density for $L / D=6$. 
As can be noted, Figures 4.17, 4.18 and 4.19 show that using the eddy ratio correction improves the prediction of the deposition density remarkably when compared to the turbulent tracking and, to some extent, when compared to the experiment although the differences that still remain. The differences can be enhanced, in addition to the isotropy assumption that is mentioned in Subsection 4.6.1, to the limitation of the eddy ratio correction. Although the eddy correction limits the production of the turbulence kinetic energy and thus the eddy viscosity especially when higher eddy ratios are used for all $L / D$ cases, it is unable to account for the near-wall features at the beginning of the wall jet region (as in the $L / D=2$ and 4 cases) and near the stagnation point (as in the $L / D=6$ case). Also, the limitation of the classical EIM that assumes the eddy velocity scale is constant during the eddy-particle interaction, affects the deposition (Galletti et al., 2008). However, overall, the eddy ratio correction for $L / D=2,4$ and 6 led to better prediction of the deposition density towards improving the large overprediction that is caused by the isotropy assumption adopted in the eddy viscosity turbulence models.

\subsection{Particle distribution over the impingement surface}

Particle distribution and deposition patterns over the impingement wall are also studied and compared. Figure 4.21 shows the particle deposition patterns using turbulent tracking (Figure 4.21a- 4.21c) and mean flow tracking (Figure 4.21d- 4.21f) for $L / D=2,4$ and 6 . The experimental deposition pattern of Burwash et al. (2006) for $L / D=2$ is shown for comparison in Figure 4.20, where the lighter regions are areas of high deposition and the darker regions are areas of low deposition. It can be noted in Figures 4.21a-4.21c that the particles are intensely deposited, especially close to the stagnation region, over the 
impingement wall for all $L / D$ cases. It can be seen the predicted pattern for $L / D=2$ is unlike the experimental pattern shown in Figure 4.20. In contrast, the mean flow tracking, where the inertial impaction is the only deposition mechanism, leads to different behavior from the turbulent tracking. Most of the particles follow the flow and a few of them are deposited on the wall as shown in Figure 4.21d- 4.21f. These results clearly indicate that the turbulent tracking, which is driven by turbulent dispersion (turbulent diffusion), is the main mechanism for the deposition of these small particles $(5 \mu \mathrm{m})$.

Figures 4.22a-4.221 show the deposition patterns for $L / D=2,4$ and 6 , using the eddy ratio correction (hybrid turbulent tracking and mean flow tracking, i.e., using $v_{T} / v=2.5,5,7.5$ and 10 for $L / D=2$ and 4 , and using $v_{T} / v=10,20,30$ and 50 for $\mathrm{L} / \mathrm{D}=6$ ). For the $L / D=2$ case, it can be noted in Figures $4.22 \mathrm{a}, 4.22 \mathrm{~d}, 4.22 \mathrm{~g}$ and $4.22 \mathrm{j}$ that as the eddy ratio is increased, the deposition is reduced and the ring-like deposition pattern appears for higher eddy ratios $\left(v_{T} / v=5,7.5\right.$ and 10 $)$ similar to the experimental pattern (Figure 4.20). It can be noted that although not quite clear, the ring pattern is still shown in Figures 4.22g $\left(v_{T} / v=7.5\right)$ and $4.22 \mathrm{j}\left(v_{T} / v=10\right)$, where most of the particles deposit in an annular region around the stagnation point that extends outward from approximately $x / D$ or $y / D \approx \pm 1$ to \pm 2 . Similarly for the $L / D=4$ and 6 cases, it is noted that as the higher eddy ratios are used, less deposition is obtained and the ringlike pattern forms around the stagnation point.

For $L / D=4$, when the eddy ratio of $10\left(v_{T} / v=10\right)$ is used, it can be noted that most of the particles deposit in an annular region around the stagnation point that extends from approximately $x / D$ or $y / D \approx \pm 0.75$ to \pm 1.5 . However, for $L / D=6$, when the eddy 
ratio of $50\left(v_{T} / v=50\right)$ is used, the ring becomes very tight such that it extends from approximately $x / D$ or $y / D \approx \pm 0.3$ to \pm 1.25 . Thus, by comparing the patterns in Figures $4.22 \mathrm{j}, 4.22 \mathrm{k}$ and 4.221 for $\mathrm{L} / \mathrm{D}=2,4$ and 6 , respectively, it can be noted that the area of the deposition density is moving towards the stagnation point as $L / D$ is increasing. This behavior is related to the nozzle-to-surface distance effect. For $L / D=2$, the highest turbulent kinetic energy is produced at the middle of the wall jet region causing more particles to deposit mainly at far distance from the stagnation point and thus causing the ring-like pattern around the stagnation point. The position of the maximum turbulent energy approaches the stagnation point as the nozzle-to-surface distance is increased leading to more deposition for the particles at the center (very close to the stagnation point) as noted for $L / D=6$ (Figure 4.221). The nozzle-to-surface distance effect is discussed in detail in the following section.

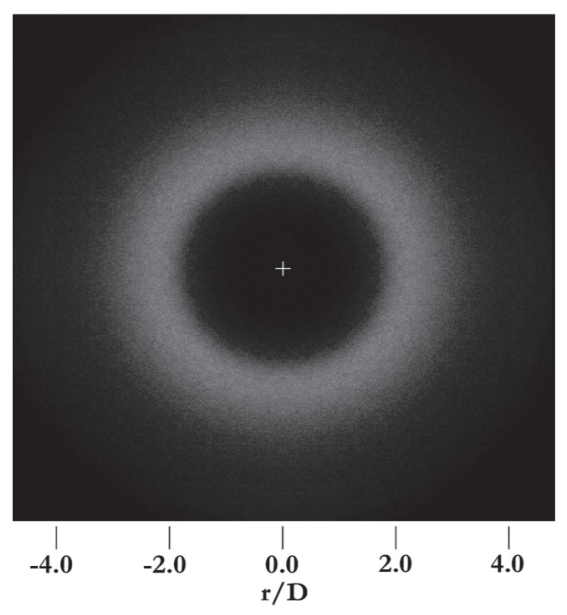

Figure 4.20. The experimental deposition pattern for $L / D=2$. (Burwash et al., 2006). 

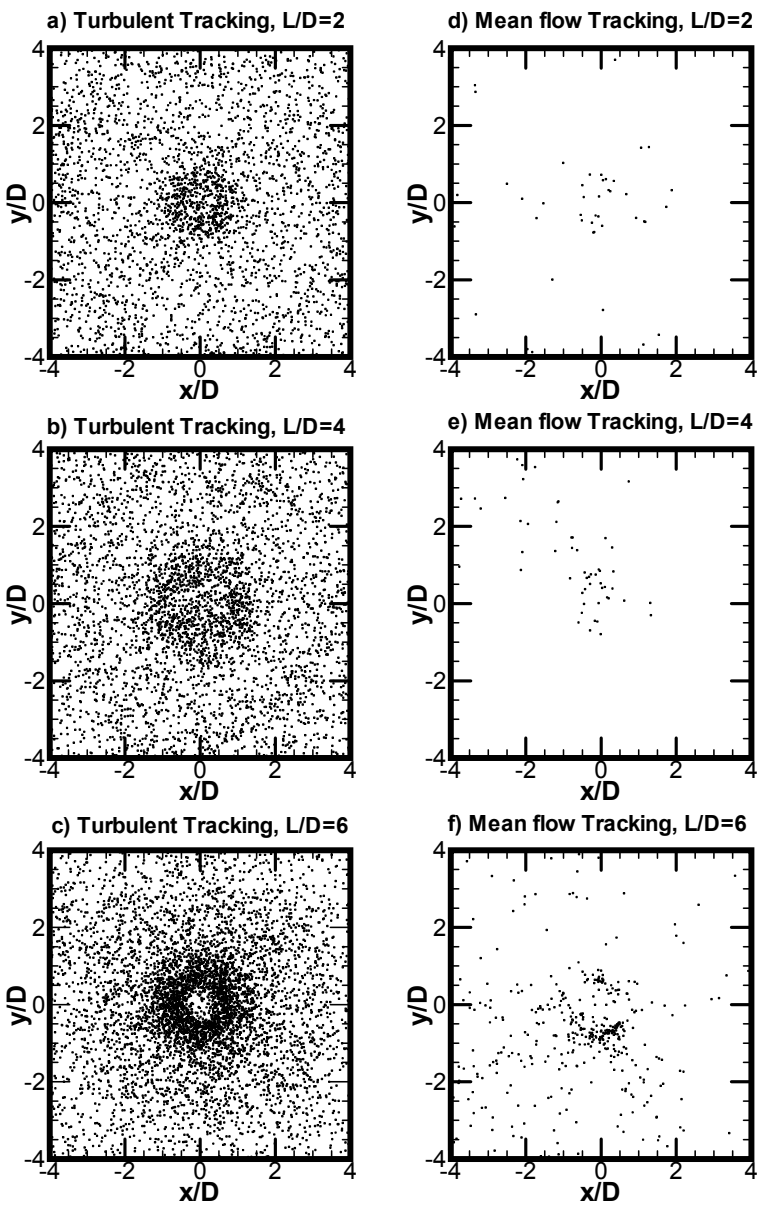

Figure 4.21. Particle deposition patterns for $L / D=2,4$ and 6 plotted for: $(a, b, c)$ turbulent tracking, and (d,e,f) mean flow tracking. 

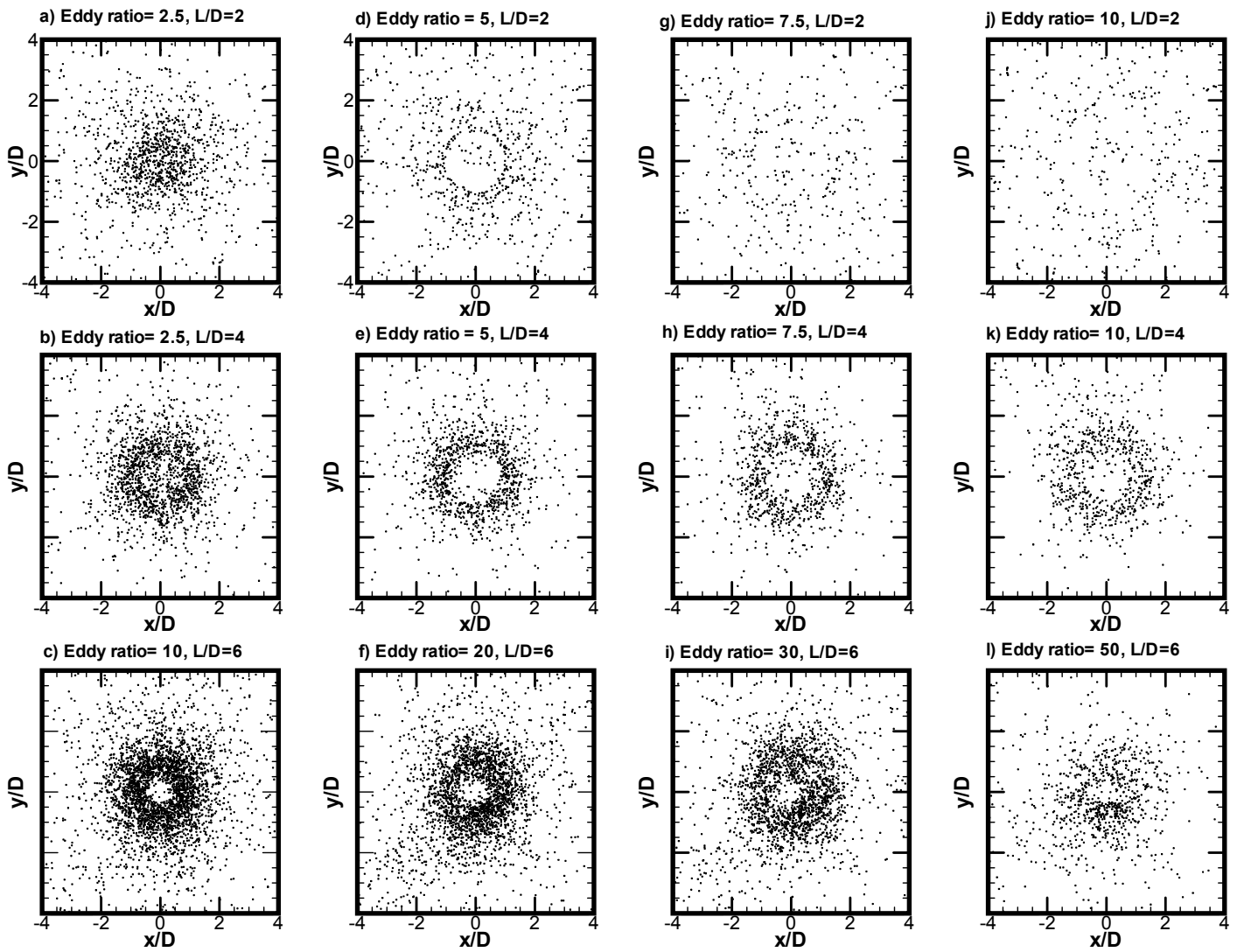

Figure 4.22. Particle deposition patterns plotted for $L / D=2,4$ and 6 .

\subsection{Effect of the nozzle-to-surface distance on particle deposition}

The influence of the nozzle-to-surface distance on the particle deposition is investigated by examining the flow field. Figure 4.23 shows the centerline velocity decay along the jet centerline axis for $L / D=2,4$ and 6 . This figure demonstrates that the $L / D=2$, and relatively, 4 cases have faster decay rate than the $L / D=6$ case. This leads to some differences in the centerline velocity as the impingement wall is approached (Burwash et al., 2006). Therefore, the larger centerline velocities for the $L / D=6$ case near the impingement wall causes the particles to be carried closer to the surface and hence to deposit due to the turbulent dispersion rather than due to inertial impaction. 
Figure 4.24 shows the turbulence kinetic energy for the $L / D=2,4$ and 6 cases along a line at a distance of $z=0.1 D$ perpendicular to the jet axis above the impingement wall. As can be noted from the figure, the turbulence kinetic energy increases as the distance $x / D$ from the centerline axis is increased, rises to different peaks for each case of $L / D$, and then begins to decay away from the centerline axis. Also, it can be noted that the level of turbulence kinetic energy in the wall jet region, i.e., at about $0.2 D \leq x \leq 1.6 D$ is increasing as the nozzle-to-surface distance $L / D$ is increased. This behavior in the turbulence kinetic energy profiles is related to the effect of the nozzle-to-surface distance.

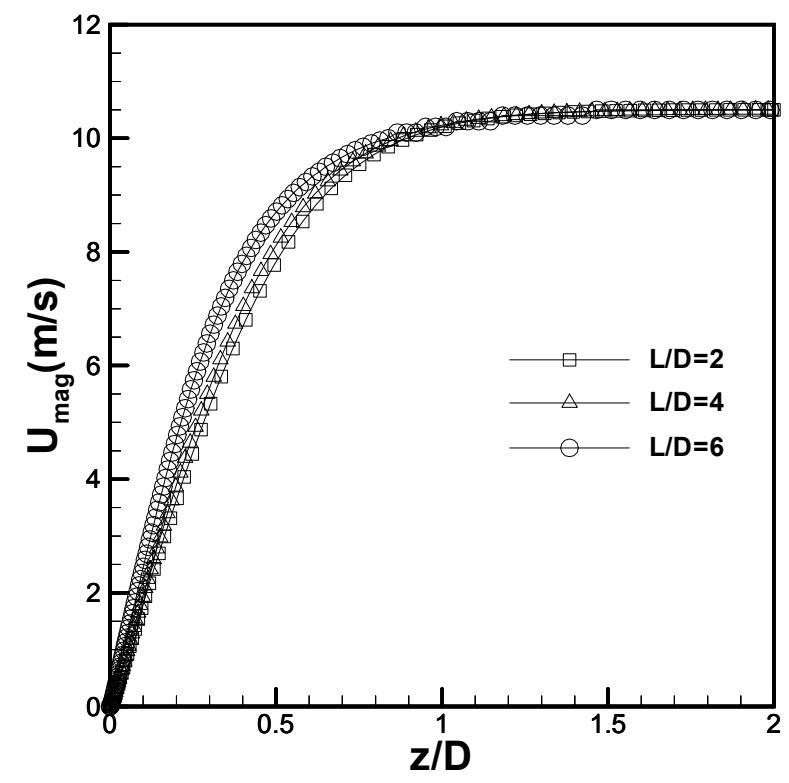

Figure 4.23. Centerline velocity along the jet axis for $L / D=2,4$ and 6 . 


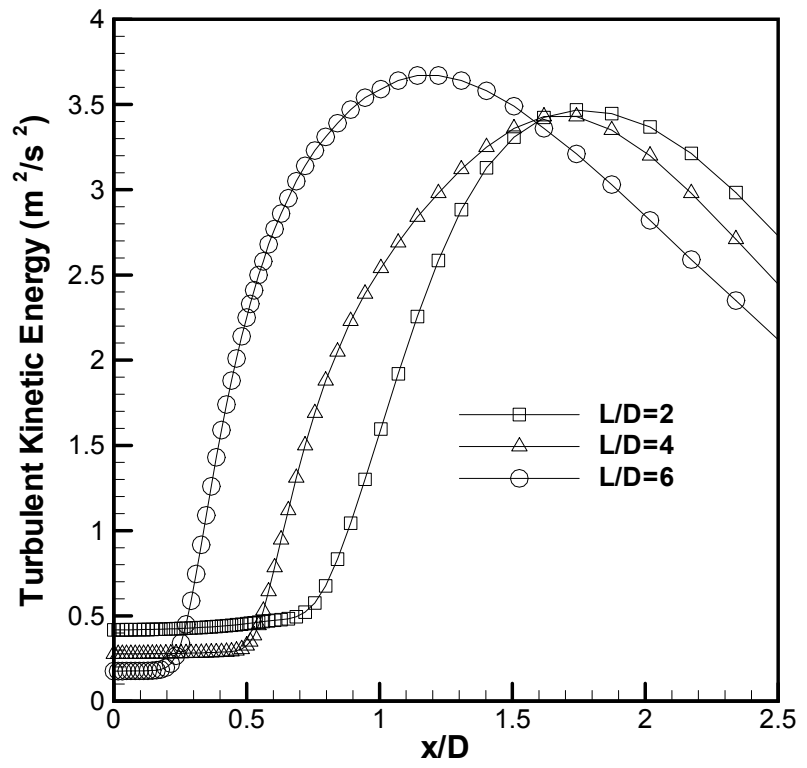

Figure 4.24. Turbulence kinetic energy along a line $(z=0.1 \mathrm{D})$ perpendicular to the jet axis for $L / D=2,4$ and 6 .

Larger nozzle-to-surface distance $(L / D=6)$ results in a greater amount of entrainment and jet spreading. Thus, more mixing occurs before the jet impinges on the wall and higher levels of turbulence (kinetic energy) results (Cooper et al., 1993 and Burwash et al., 2006). This causes large number of particles to deposit in this region for the $L / D=6$ case compared to the $L / D=4$ and 2 cases.

Figures $4.25 \mathrm{a}-4.25 \mathrm{~d}$ show the turbulence kinetic energy profiles along lines $x=$ $0.0 D, 0.5 D, 1.0 D$ and $2.0 D$, respectively. In Figure $4.25 \mathrm{a}$, in the stagnation region, there is low level of turbulence kinetic energy for the three nozzle-to-surface distances. This is because the flow is deflected by the wall in this region, and thus low energy is produced. However, as the flow is developed further downstream at $x=0.5 D$ and $x=1.0 D$, the level of the kinetic energy increases as the nozzle-to-surface distance is increased in a region 
very close to the wall $(z<0.4 D)$. The reason of this increase in energy for the three cases is because of the wall jet that starts to develop around these stations $(x=0.5 D$ and $x=$ $1 D$ ) and hence contributes to high levels of energy within this region. It can be noted in these two stations that the $L / D=6$ case has higher levels of turbulence kinetic energy than $L / D=4$ and 2. This is because longer development region occurs in $L / D=6$, which allows for more mixing with ambient air (Burwash et al., 2006). Further downstream, at $x$ $=2 D$, the three $L / D$ cases show close energy levels.

These results suggest that the deposition on the impingement wall, for the three $L / D$ cases, occurs in regions close to the regions of high turbulence kinetic energy. Also, these results suggest that the deposition in the case of $L / D=6$ is higher than in the $L / D=2$ and 4 cases, due to the significant increase in the turbulence kinetic energy, which occurs in regions close to the stagnation region (i.e., $x / D=0.5$ and $x / D=1$ ).

Figure 4.26 shows contours of the development of the magnitude of the mean velocity and turbulence kinetic energy for the $L / D=2,4$ and 6 cases. As can be seen in Figures $4.26 \mathrm{a}-4.26 \mathrm{c}$, although the three cases appear to have similar development, they differ in the extent of the development stage. The flow in shorter nozzle-to-surface distance has less chance to spread before impinging on the surface (for $L / D=2$ and, at lesser degree for, $L / D=4$ ), and the jet centerline velocity decays faster unlike the $L / D=6$ case, in which the velocity is more developed. For all $L / D$ cases, as the impingement surface is approached and the flow is deflected, the wall jet starts to develop and high levels of velocity and kinetic energy are produced. 
The development of the kinetic energy as shown in Figures 4.26d-4.26f is associated with the velocity development. As a result, this leads to differences in the behavior of kinetic energies in the wall jet region (parallel to the impingement surface), which consequently influences the particle deposition. Therefore, more deposition occurs near the stagnation point for the $L / D=6$ case than for the $L / D=2$ and 4 cases. This is because the maximum kinetic energy is produced around $x / D=0.5 \sim 2$ for the $L / D=6$ case, which is different from the range of maximum kinetic energy at approximately $x / D$ $=1.5 \sim 2.5$ and $x / D=1 \sim 2.25$ for the $L / D=2$ and 4 cases, respectively, as shown in Figures 4.26d-4.26f. 
a) $x / D=0$

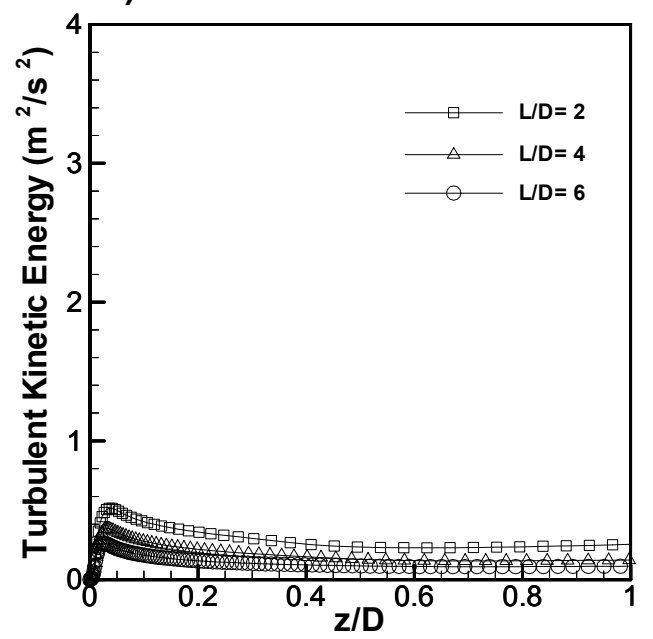

c) $x / D=1$

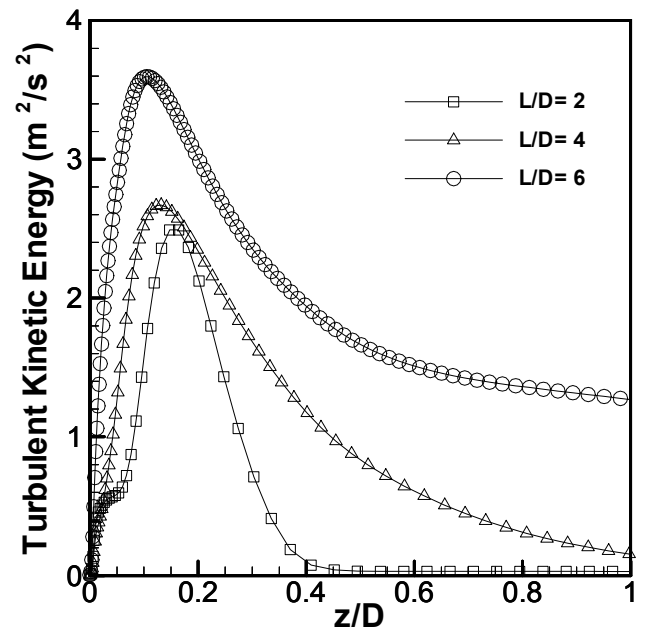

b) $x / D=0.5$

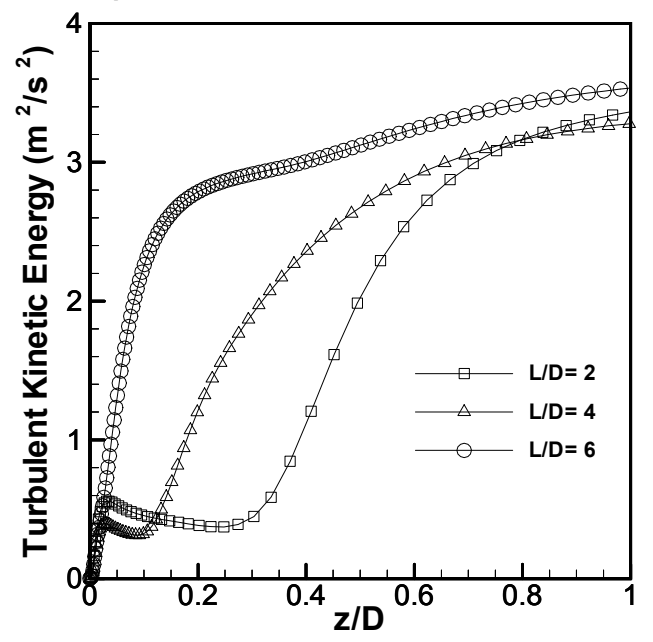

d) $x / D=2$

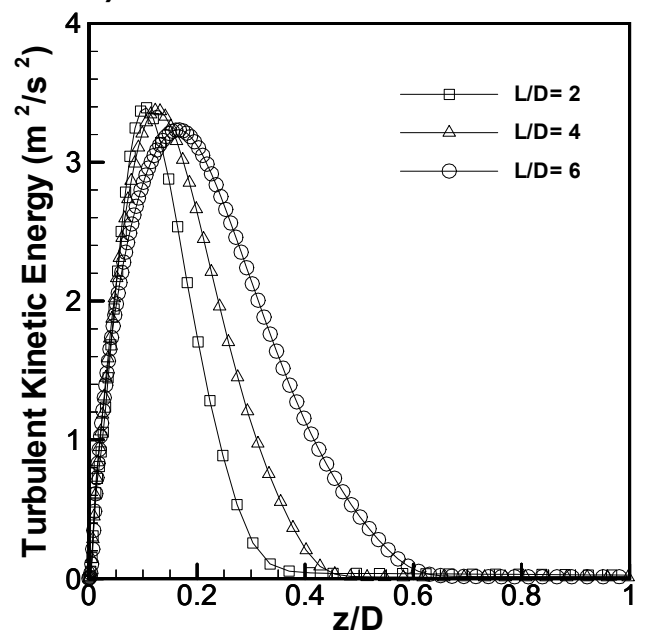

Figure 4.25. Turbulence kinetic energy at distances from the jet axis: (a) $x=0 D$, (b) $x=0.5 \mathrm{D}$, (c) $x=1.0 \mathrm{D}$, and (d) $x=2.0 \mathrm{D}$ for $\mathrm{L} / \mathrm{D}=2,4$ and 6 . 

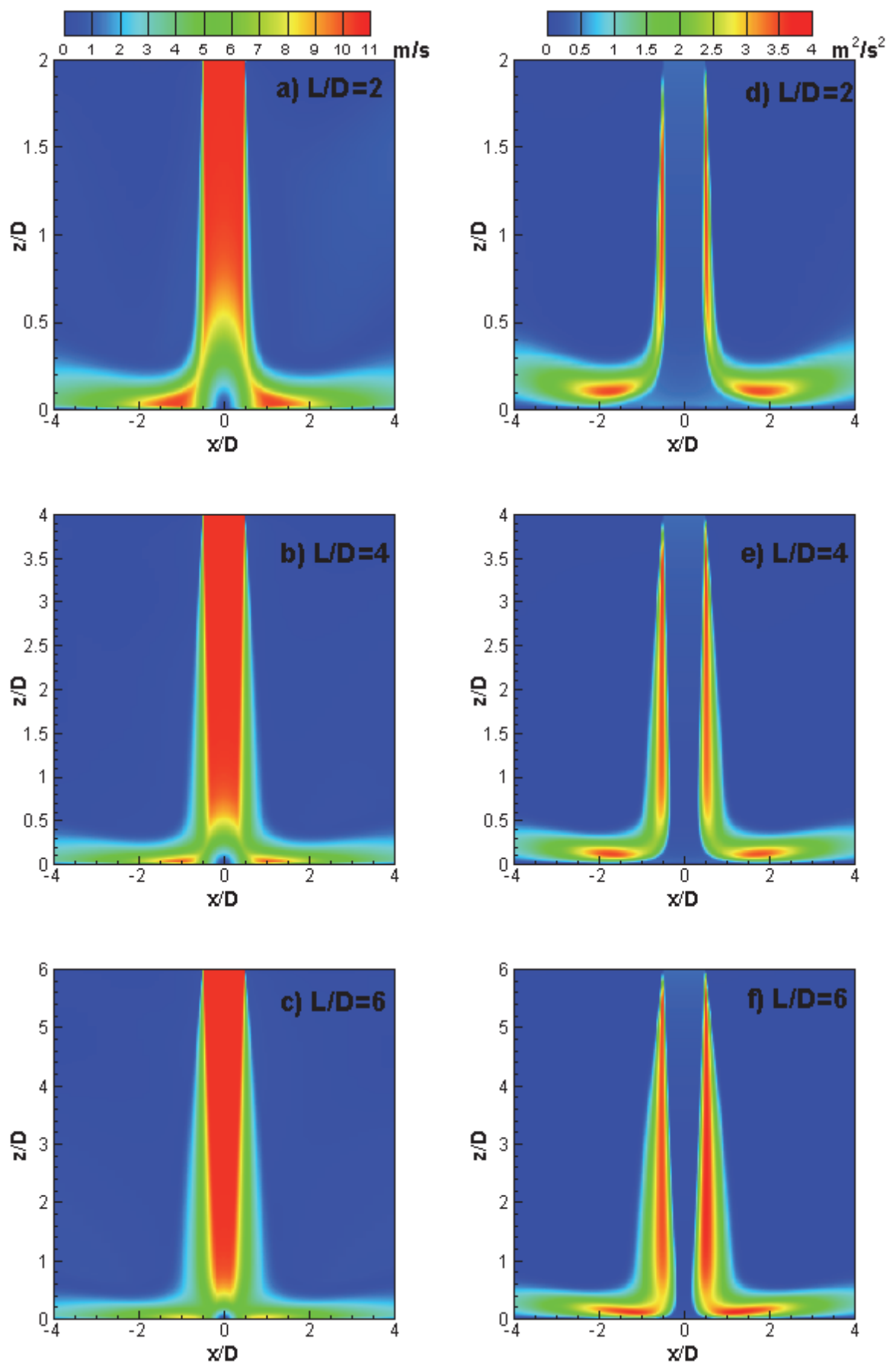

Figure 4.26. Contours ( $x-z$ plane, $y=0$ ) of the magnitude of the mean velocity (a-c), and turbulence kinetic energy (d-f) for $L / D=2,4$ and 6 . 


\subsection{Deposition results using modified EIM}

\subsubsection{Turbulent tracking and mean flow tracking}

Particle deposition using modified EIM, which was introduced in Subsection 3.3.1.2 and employed in the developed tracking code, is investigated. Figures 4.27, 4.28 and 4.29 show the particle deposition density for the three nozzle-to-surface distances of $L / D=2$, 4 and 6, respectively, plotted versus $r / D$ using turbulent tracking and mean flow tracking. As can be seen from Figures $4.27(L / D=2)$ and $4.28(L / D=4)$ that while the deposition density is overpredicted by turbulent tracking in region $r / D<1.6$ for $L / D=2$ when compared to the experiment of Burwash et al. (2006), it is also overpredicted over the entire range of $r / D$ for $L / D=4$.

For $L / D=6$ (Figures 4.29), the deposition density using the turbulent tracking is also overpredicted in region $0.3 \leq r / D \leq 2.6$ and underpredicted very close to the stagnation point $(r / D<0.3)$ although it has a better prediction beyond $r / D=2.6$. The overprediction in these figures is due to the isotropic decomposition effect cited in Subsection 4.6.1. For the mean flow tracking, the deposition density is underpredicted for all the cases $(L / D=2,4$ and 6$)$, as expected, and few particles are deposited near the stagnation point.

The above results indicate, as stated in Subsection 4.6.1, that the turbulent dispersion is the main mechanism for the deposition of the particles over the impingement wall. This is applicable for small particle sizes, which is considered in the present research, where the Stokes number, with $d_{p}=5 \mu \mathrm{m}$ and $\mathrm{Re}=10000$, is equal to 0.11 . This Stokes number is in the range where the particles follow the rapid change in 
the fluid and respond quickly to the behavior of the fluid phase. However, as the Stokes number is increased by using larger particles (larger diameters), other mechanisms for the deposition such as gravity and inertial impaction become more important than the turbulent dispersion. These mechanisms are significant for larger Stokes number around $S t k \approx 1$ or larger (Finlay, 2001) because particles with Stokes number in this range have no tendency to follow the flow closely and probably cause more deposition. Increasing the Reynolds number at the same particle size of $5 \mu \mathrm{m}$ may increase the deposition (Abuzeid et al., 1991) due to the increase in the turbulent fluctuations, which in turn increases the turbulent dispersion.

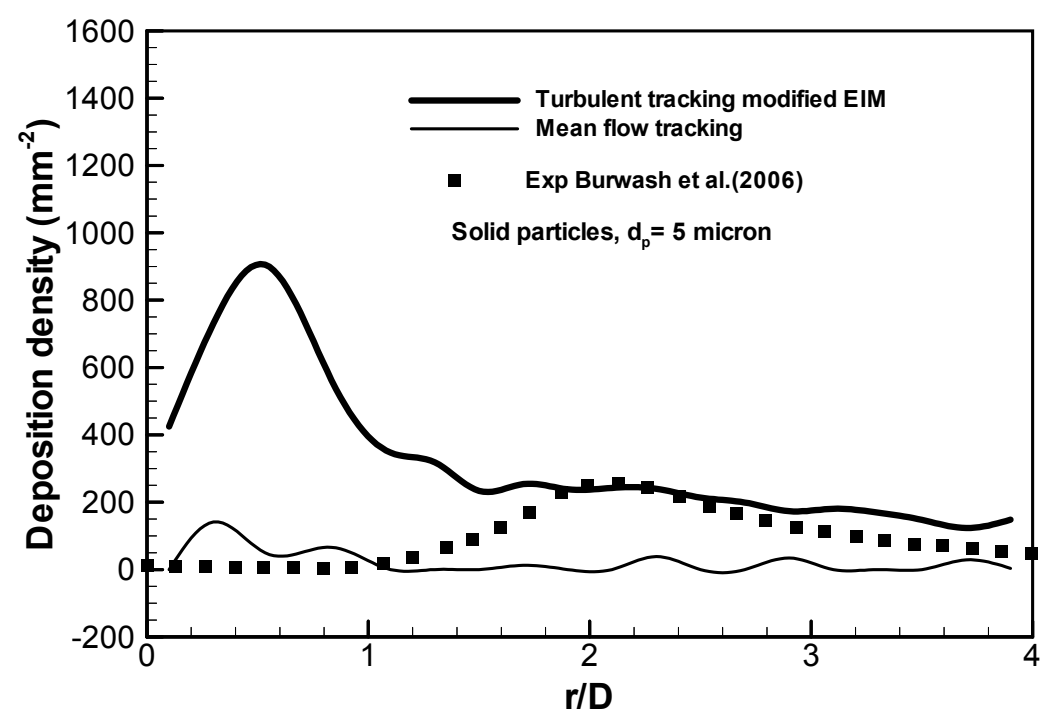

Figure 4.27. Deposition density using turbulent tracking (modified EIM) and mean flow tracking for $\mathrm{L} / \mathrm{D}=\mathbf{2}$. 


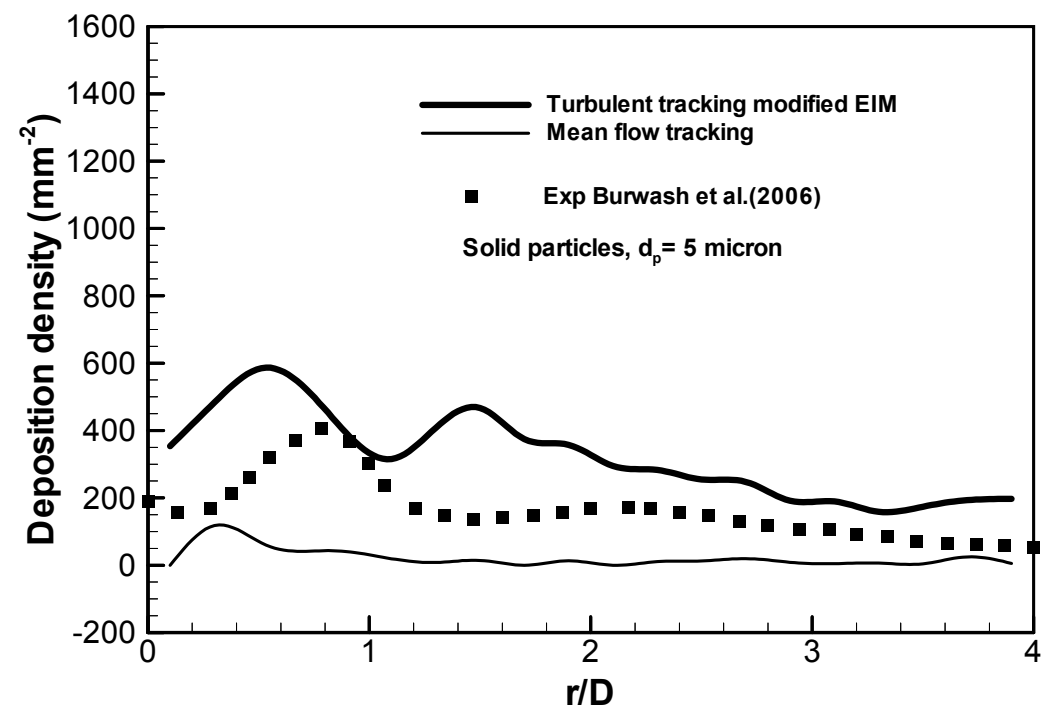

Figure 4.28. Deposition density using turbulent tracking (modified EIM) and mean flow tracking for $L / D=4$.

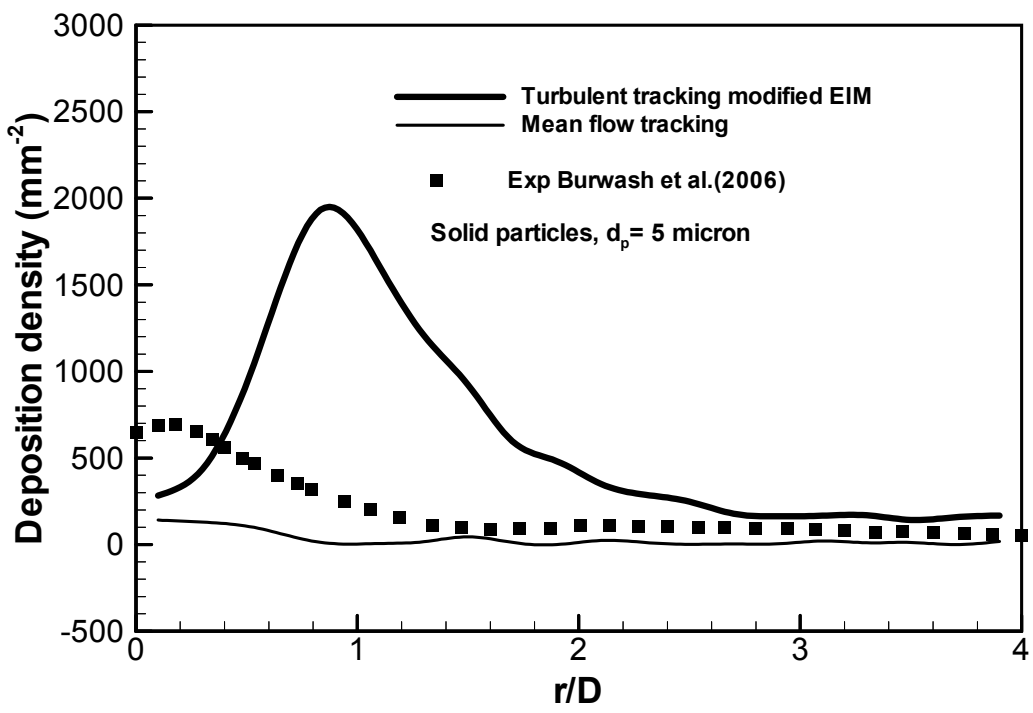

Figure 4.29. Deposition density using turbulent tracking (modified EIM) and mean flow tracking for $L / D=6$.

It is worth noting from Figures $4.27,4.28$ and 4.29 that the particle deposition density obtained from modified EIM using turbulent tracking has lower levels and 
generally a better prediction than the deposition density that is obtained from classical EIM. This difference between classical EIM and modified EIM is because in modified EIM, the RMS fluid fluctuating velocity (eddy velocity scale) is allowed to vary with the particle position during the eddy-particle interaction. In the classical EIM, which is available in CFX tracking code, the RMS fluid fluctuating velocity is constant during the interaction. This constant fluctuating velocity results in an unrealistic larger dispersion of particles and thus a larger deposition than the case when varying fluctuating velocity is used as in modified EIM.

\subsubsection{Near-wall correction}

In turbulent flow, the turbulent dispersion (i.e., by the fluid flow fluctuations) is the main mechanism for particle deposition, especially for small particle sizes $(5 \mu \mathrm{m}$ and $S t k=$ 0.11). Therefore, the appropriate modeling of the fluid fluctuations in the near-wall region is crucial for accurate particle simulations. It is known that, the RANS SST and k$\omega$ turbulence models in the CFX were formulated for isotropic decomposition of the turbulence kinetic energy $\left(u_{f r m s}^{\prime}=v_{f r m s}^{\prime}=w_{f r m s}^{\prime}=(2 k / 3)^{0.5}\right)$. The use of $(2 k / 3)^{0.5}$ would give overprediction for the normal-to-wall fluctuating velocity component, which is the main component responsible for particle deposition on the impingement wall, near the stagnation point and in the wall jet region (Nishino et al., 1996). As a result, the overprediction of the normal-to-wall fluctuating velocity will cause an overprediction in the deposition as was shown in Subsections 4.6.1 and 4.9.1.

For this reason, some improvements are needed to account for the near-wall features in the near-wall region. The near-wall damping functions are practical means to 
account for the influence of the wall on the fluid flow fluctuations, and to avoid all the complexities of the sublayer region. Therefore, to improve the prediction of the particle deposition in the impinging jet flow, the near-wall correction is used in the present study. This can be accomplished by applying the near-wall correction functions that are incorporated into the tracking code that is developed in the present research.

In general, the velocity fluctuation details near the wall are quite limited. In the literature, most of these details are obtained from channel (or pipe) flow for which a lot of experimental data and empirical equations are available. In addition, the DNS data, which can capture the primary flow representation including the velocity fluctuations near the wall, is also available such that several near-wall damping functions were developed based on DNS pipe flow data. Conversely, it should be mentioned here that for impinging jet flow the reliable near-wall turbulence data are absent in stagnation and wall jet regions.

For an accurate analysis of the particle transport and deposition process, a new damping function is proposed in the present research. This function is based on the Van Driest (1956) damping factor which is given by the following function

$$
f=1-e^{\frac{-y^{+}}{A}}
$$

where $A$ is a constant that has to be determined. The dimensionless wall distance $\mathrm{y}^{+}$is calculated using the formula $\mathrm{y}^{+}=\mathrm{y} u^{*} / v$, where $u^{*}=\sqrt{\tau_{w} / \rho}$. Here, $\tau_{\mathrm{w}}$ is the wall shear stress, and $y$ is the normal distance to the impingement wall (identical to $z$ in the present research). Before applying this function in the present analysis, it should be calibrated 
such that it gives the most accurate representation of the normal-to-wall velocity fluctuation, which has significant effect on the particle deposition, based on comparison with previous experimental data (Cooper et al., 1993). This can be obtained by determining the value of $A$ in the function, based on firm physical considerations, according to the impinging jet flow characteristics. To accomplish this, careful numerical experiments and comparisons should be established between calculations and experiments pertaining to impinging jet flow. For this reason, series of numerical simulations for the primary flow (single-phase), under conditions similar to the experiment of Cooper et al. (1993), were performed for nozzle-to-surface distances of $L / D=2$ and 6 as described before in Section 4.1. The experiment of Cooper et al. (1993) is the most cited experimental paper in the literature on the subject of impinging jet flow. This experiment reported several flow characteristics of the impinging jet flow for various nozzle-to-surface distance cases although some of them, such as $L / D=4$, were not reported.

As described in Section 4.1, separate computational domains were generated for $L / D=2$ and 6 based on the experiment of Cooper et al. (1993). The grid convergence analysis was performed and is presented in Appendix C.

Before calibrating the correction function, the accuracy of the fluid flow simulation was examined by validating its predictions for the mean and fluctuating velocities with the experimental data of Cooper et al. (1993). Also, by comparing with previous simulation of Kubacki and Dick (2010b) (for $L / D=2$ ) and with the simulation of Craft et al. (1993) (for $L / D=6$ ). This is described in the following subsection. 


\subsubsection{Fluid flow results}

The profiles of the mean velocity magnitude and the fluctuating velocity, along lines perpendicular to the impingement wall, versus the dimensionless distance from the wall $y / D$ are shown, respectively, in Figures $4.30 \mathrm{a}-4.30 \mathrm{c}$ and $4.30 \mathrm{~d}-4.30 \mathrm{f}$, for $L / D=2$. The simulation results are obtained using the SST model with Kato and Launder modification (shown by open delta) (Kato and Launder, 1993) and also without Kato and Launder modification (shown by open square). The mean velocity magnitude and the fluctuating velocities are normalized with the bulk velocity of the jet $U_{B}$. As can be noted from Figures $4.30 \mathrm{a}-4.30 \mathrm{c}$, the profiles of the magnitudes of the mean velocity with and without Kato and Launder modification have good prediction as the flow moves from the stagnation region to the wall jet region, i.e., from $x / D=0.5$ to $x / D=2.5$. Although the slight difference between the present simulations and the experimental data of Cooper et al. (1993) (dark square symbols), it can be noted that the predicted profiles behave closely as the experiment near the impingement wall and in the outer part of the wall jet. Also, it can be noted that there is agreement between the present simulation and the simulation data of Kubacki and Dick (2010b) (shown by open circles).

The fluctuating velocities have similar trends as in the experiment although the overestimation noted as the flow moves downstream from $x / D=0.5$ to $x / D=2.5$. However, it can be noted that the fluctuating velocity at $x / D=0.5$ obtained with Kato and Launder modification has qualitatively close prediction as the experiment in spite of the overestimation very close to the impingement wall. In general, the present simulation results are consistent with the previous simulation of Kubacki and Dick (2010b). 
a)

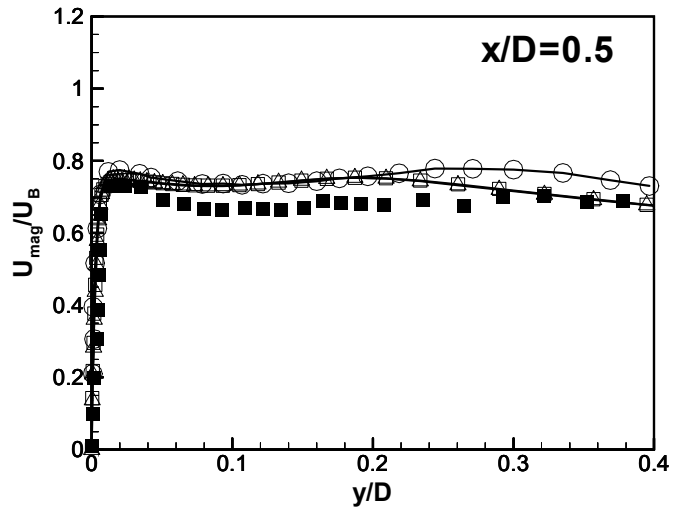

b)

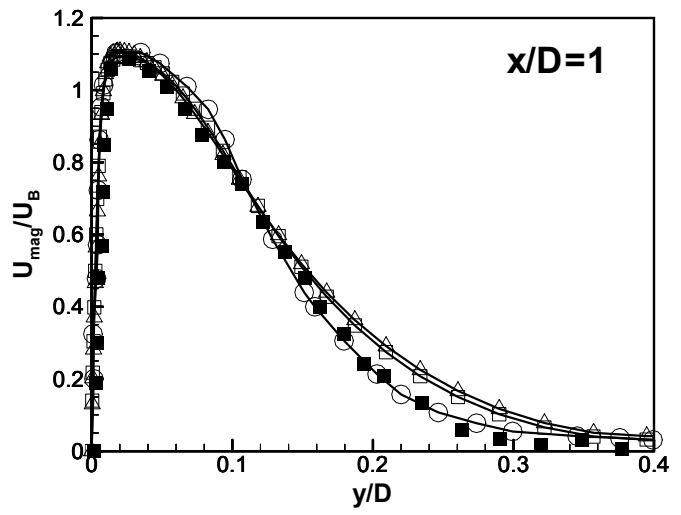

c)

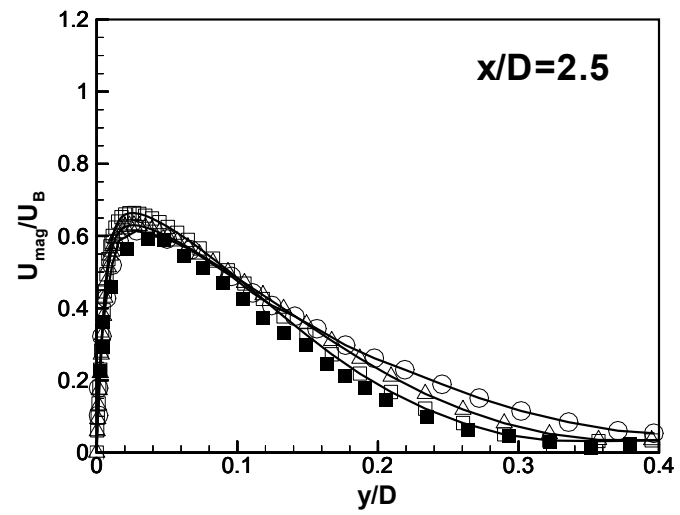

d)

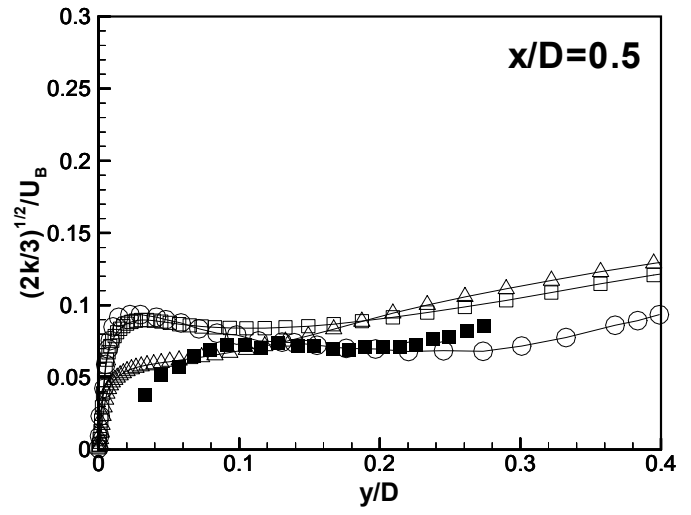

e)

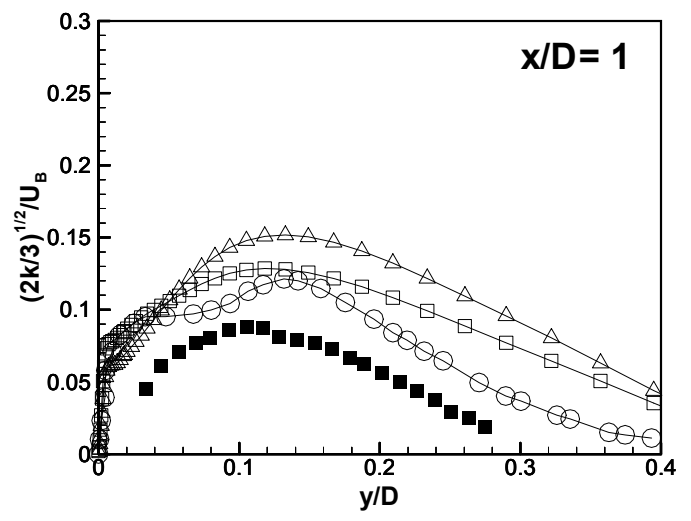

f)

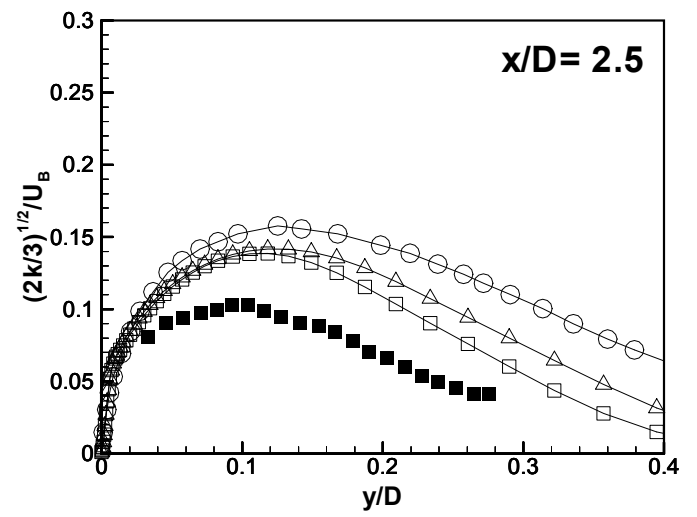

Figure 4.30. Mean velocity magnitude (a-c), and normal-to-wall RMS (d-f) for $L / D=$ 2 at three stations: $x / D=0.5,1$ and 2.5 . 
a)

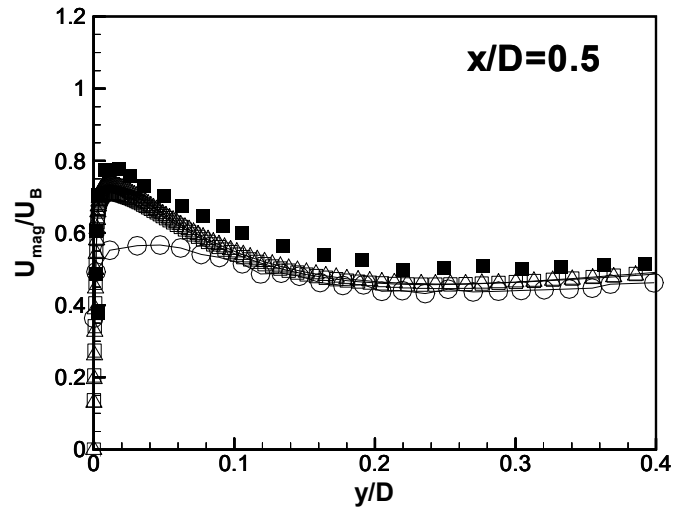

b)

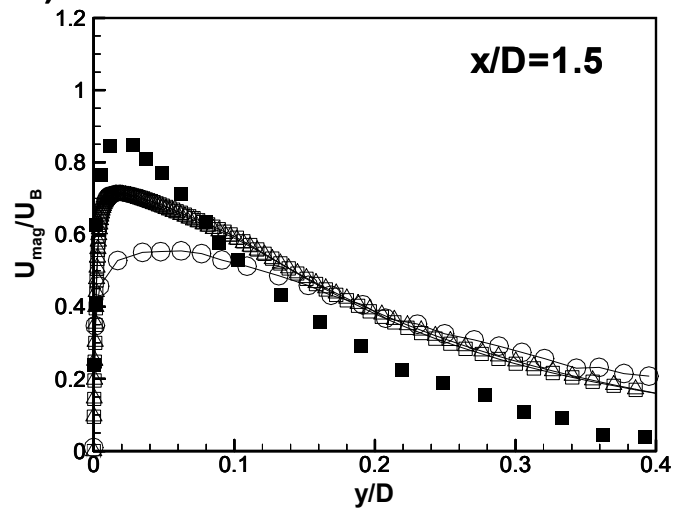

c)

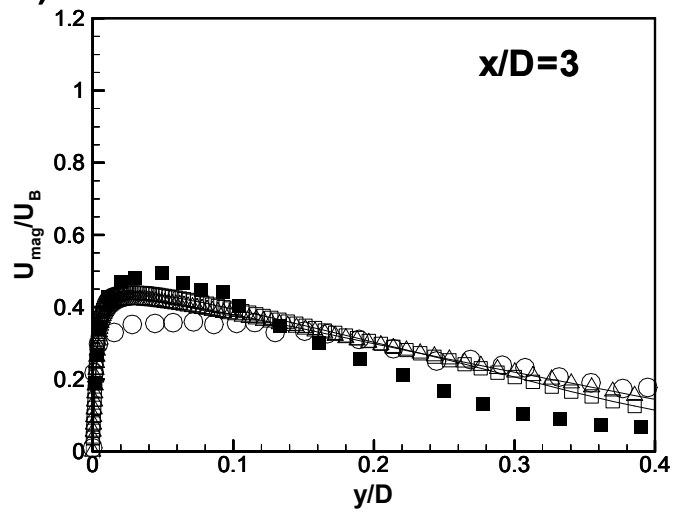

d)

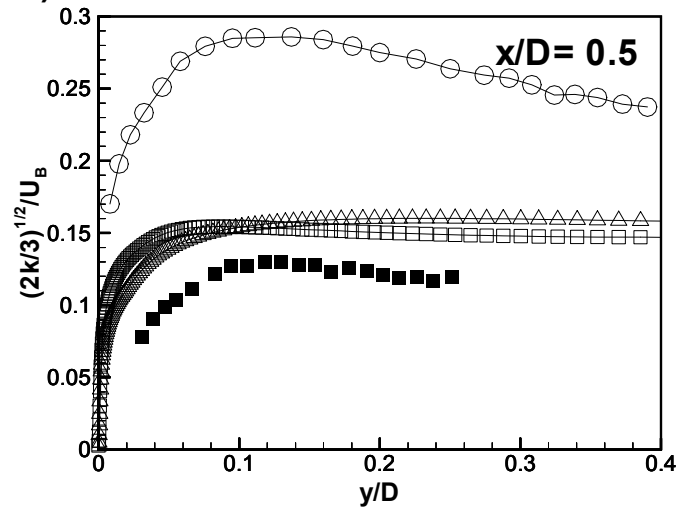

e)

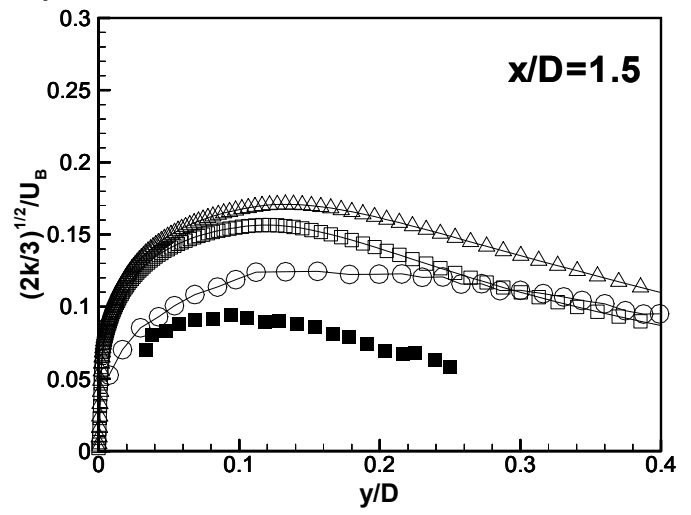

f)

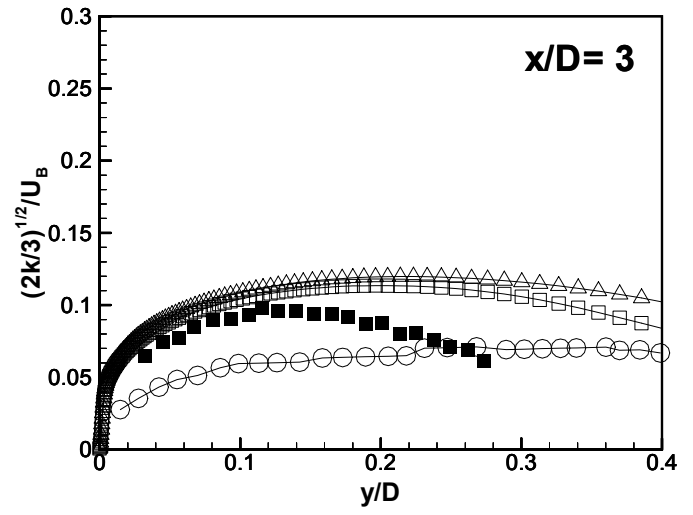

Figure 4.31. Mean velocity magnitude (a-c), and normal-to-wall RMS fluctuation (df) for $L / D=6$ at three stations: $x / D=0.5,1.5$ and 3 . 
Similarly for $L / D=6$ as shown in Figures $4.31 \mathrm{a}-4.31 \mathrm{c}$, the profiles of the magnitude of the mean velocity, which are obtained with (open delta) and without (open square) Kato and Launder modification, are close to the experiment (dark square symbols) at $x / D=0.5$ and 3 . However, at $x / D=1.5$, which is a region of strong flow acceleration, the mean velocity has a lower peak than the experiment and a higher velocity at large $y / D$ values. This difference can be attributed to the rapid mixing of the flow, which occurs as the flow accelerates strongly from the stagnation region to the wall jet region, as predicted by the turbulence model (Craft et al., 1993). Generally, the main features of the flow, such as the decay of the mean velocity in the viscous sublayer and the spreading of the mean velocity into the free stream, are captured as shown in Figures 4.31a-4.31c. The present simulation is consistent with the simulation of Craft et al. (1993) (open circles). The fluctuating velocities obtained with and without Kato and Launder modification have similar trends as in the experiment. However, they are overestimated when compared to the experiment for all the perpendicular lines as shown in Figures 4.31d-4.31f. The previous simulation of Craft et al. (1993) also shows an overprediction, with the exception of $x / D=3$, where there is an underprediction. The physical phenomena behind the trends of the mean and fluctuating velocities shown in Figures 4.30 and 4.31 are similar to those described in Section 4.5.

The overprediction in the normal-to-wall fluctuating component, which is shown for $L / D=2$ and 6 (Figures 4.30 and 4.31) can be attributed to the excessive prediction of the turbulence kinetic energy (Craft et al., 1993 and Kubacki and Dick, 2010b). This excessive prediction in the turbulence kinetic energy takes place in the wall jet and stagnation regions as obtained from the SST model, although the stress limiter employed 
in the model and Kato and Launder modification. In addition, the SST model is insensitive to the streamline curvature effect due to the eddy viscosity assumption used in the model. Therefore, it is necessary to predict the RMS normal-to-wall fluctuating velocity to an adequate level for an accurate particle deposition prediction. This can be obtained by calibrating the present RMS normal-to-wall fluctuating velocity using nearwall correction function, in order to yield the best fit with the experiment, and then using it in the deposition analysis as shown in the following subsection.

\subsubsection{Deposition results}

As mentioned above, it is important to use the function based on the impinging jet flow characteristics to account for the near-wall effects. In other words, the normal-to-wall RMS fluctuating velocity that causes the particles to deposit on the impingement wall should be represented accurately by conducting a critical evaluation. In the light of lack of turbulence data for the impinging jet flow near the wall (the wall jet region and the stagnation point), the accurate representation of the normal-to-wall RMS fluctuating velocity is based upon the comparison between calculations and the available experiment. Therefore, this is carried out by comparing the normal-to-wall RMS fluctuating velocity, which will be corrected using Equation (4.3), with the experimental data of Cooper et al. (1993) at different stations. Using Equation (4.3), the corrected normal-to-wall RMS fluctuating velocity is obtained as

$v_{f, r m s, \text { corr }}^{\prime}=\left(1-e^{\frac{-y^{+}}{A}}\right)(2 k / 3)^{0.5}$

The determination of the value $A$ in Equation (4.4) is described in the next paragraphs. 
In the present research, in order to determine the value of $A$, two different procedures were implemented. The first procedure is based on choosing a value for $A$, which has the closest agreement with the experimental data at every station, and then taking the average value of them and hence using this average value in the correction function. The second procedure relies on the best fit basis. In other words, the value of $A$ that gives the closest fit to the experiment overall the stations is chosen and used in the correction function. These two procedures are demonstrated by plotting several values of $A$ along with the experimental data of Cooper et al. (1993) (dark square symbols) as shown in Figures 4.32 and 4.33.

Figures $4.32 \mathrm{a}-4.32 \mathrm{c}$ and $4.32 \mathrm{~d}-4.32 \mathrm{f}$ show the normal-to-wall RMS fluctuating velocity at different stations versus $y / D$ using the SST model, without and with Kato and Launder modification, respectively, for $L / D=2$. The normal-to-wall RMS fluctuating velocity without damping, i.e., without near-wall correction (open square symbol) as obtained from RANS SST model is shown along with the corrected normal-to-wall RMS fluctuating velocity using Equation (4.4) for different values of $A$. As can be seen in the figures, the normal fluctuating velocity without damping demonstrates high levels, even with Kato and Launder modification, as the impingement wall is approached. This causes overprediction in the deposition when turbulent tracking was used. As the correction with Equation (4.4) is used in the near wall region, the normal-to-wall RMS fluctuating velocity is damped.

In Figure 4.32, it can be noted that the average value of $A=76$ for the case without Kato and Launder modification across the stations $x / D=0.5$ to 1 (right triangle symbols, 
Figure 4.32a-4.32c), which is determined using the first procedure, still gives higher values of the normal-to-wall RMS fluctuations with respect to the experimental data despite the noted correction. This is because the normal-to-wall RMS fluctuation has different level at every station. Also, the average value of $A=63$ (right triangle symbols, Figure 4.32d-4.32f) with Kato and Launder modification, although the slight agreement at $x / D=0.5$ with the experimental data, it has overestimation at $x / D=1$, where this region is high kinetic energy region for $L / D=2$. Therefore, the first procedure does not lead to a value of $A$ that is very close to the experimental data across all the stations. With the second procedure, the value of $A=100$ (diamond symbols) gives the closest fit to the experiment (Cooper et al., 1993) overall the stations for the cases with and without Kato and Launder modification although the unavoidable underprediction downstream the wall jet region at $x / D=2.5$. The second procedure leads to the best compromising value of $A$ across the near-wall region. Using this procedure leads to a better value of $A$ that can give an adequate level of the normal-to-wall fluctuation (best fit to the experiment) in the regions $(x / D=0.5$ and 1$)$ of excessive overprediction of turbulence kinetic energy. Therefore, this leads to a marked reduction in the deposition density as will be shown later in this section.

In the same way, for $L / D=6$ as shown in Figures $4.33 \mathrm{a}-4.33 \mathrm{f}$, the normal-to-wall RMS fluctuating velocity is lowered as Equation (4.4) is used for several values of $A$. As can be shown in the figures, the first procedure, using the average value of $A=150$ (for the case without Kato and Launder modification, right triangle symbols) and the average value of $A=141$ (for the case with Kato and Launder modification, delta symbols), gives slightly higher levels of the normal-to-wall fluctuations compared to the experiment alth- 
a) $x / D=0.5$

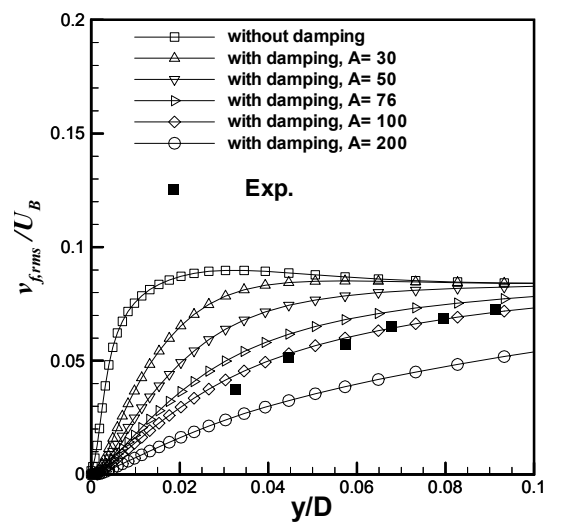

b) $x / D=1$

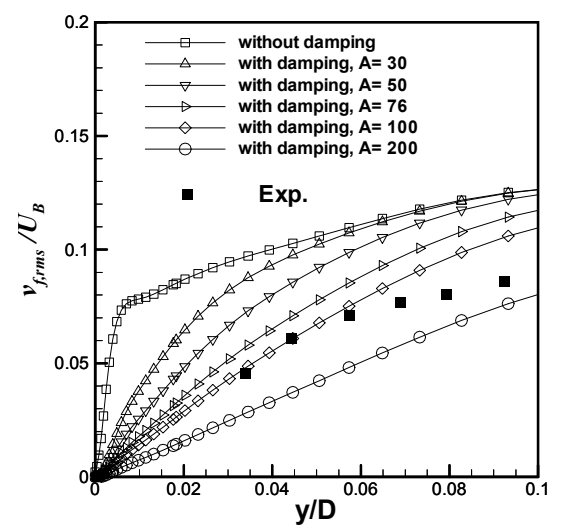

c) $x / D=2.5$

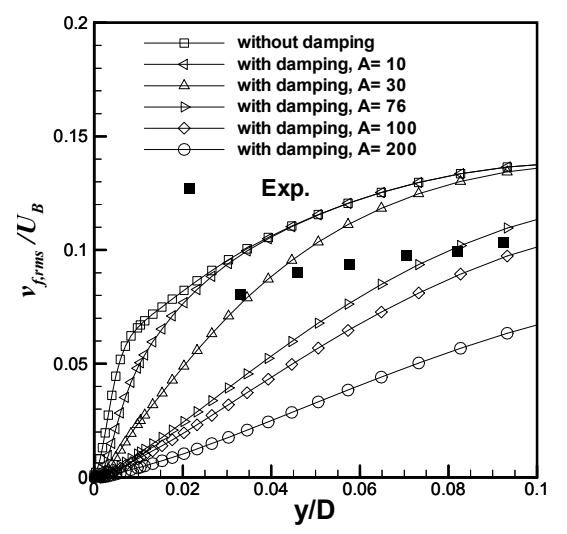

d) $x / D=0.5$

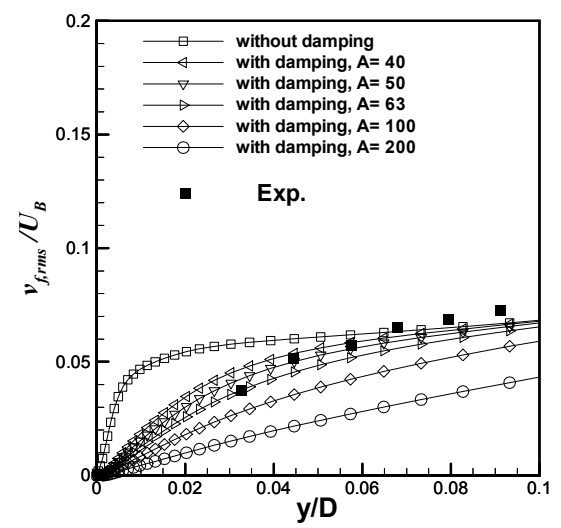

e) $x / D=1$

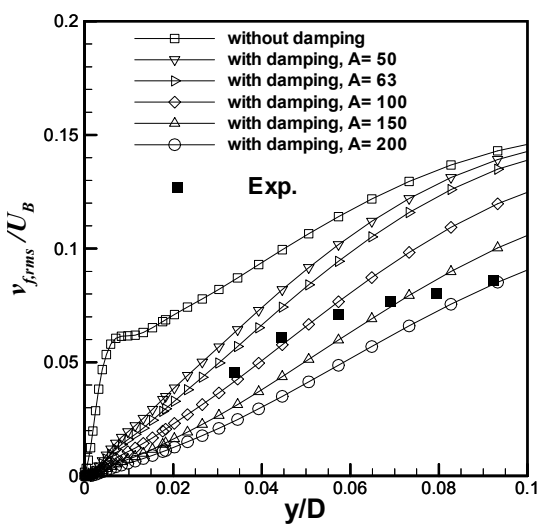

f) $x / D=2.5$

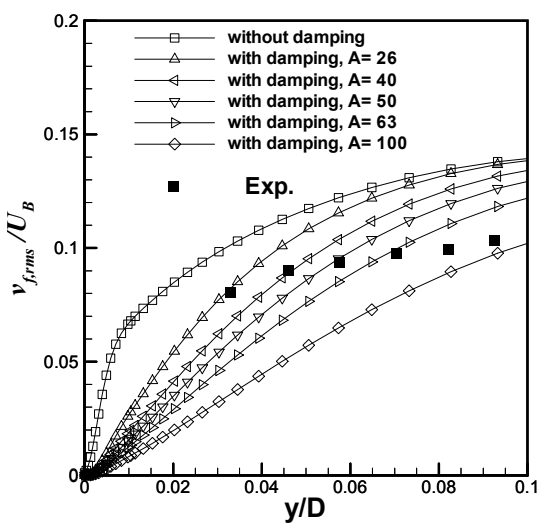

Figure 4.32. Calibrating normal-to-wall RMS fluctuation using SST model without Kato and Launder modification (a-c), and with Kato and Launder modification (df) for $L / D=2$ at three stations: $x / D=0.5,1$ and 2.5 . 
a) $x / D=0.5$

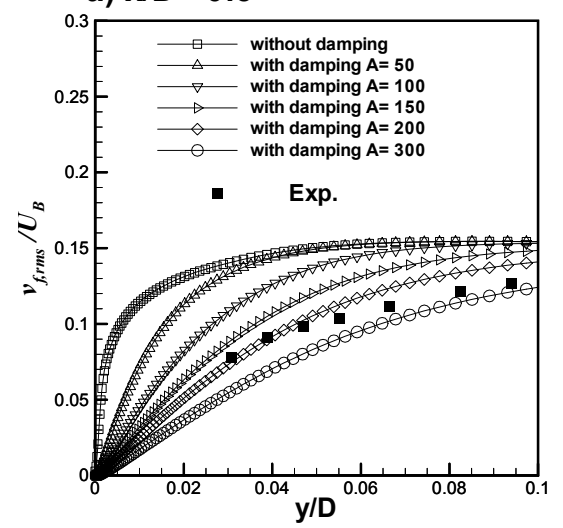

b) $x / D=1.5$

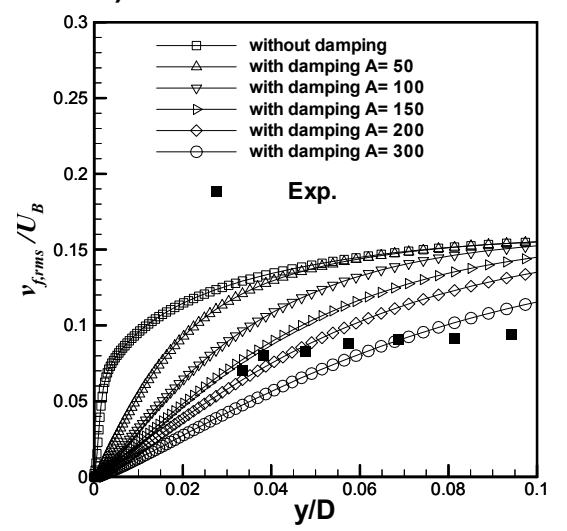

c) $x / D=3$

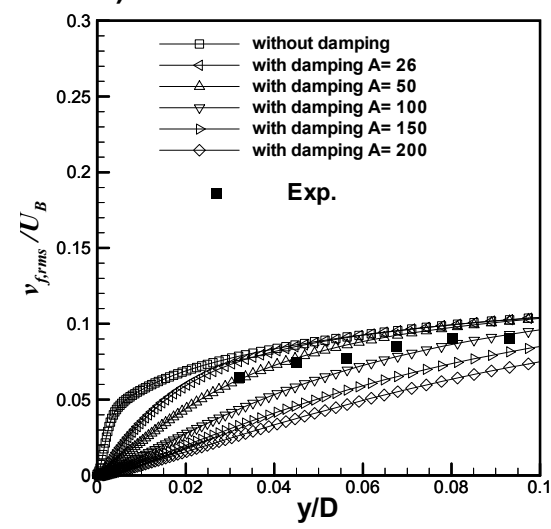

d) $x / D=0.5$

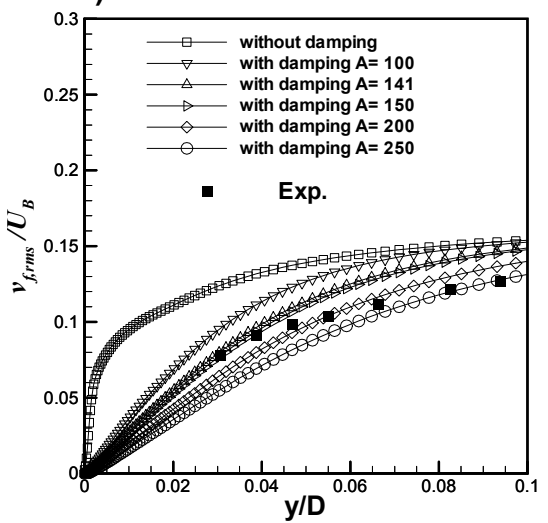

e) $x / D=1.5$
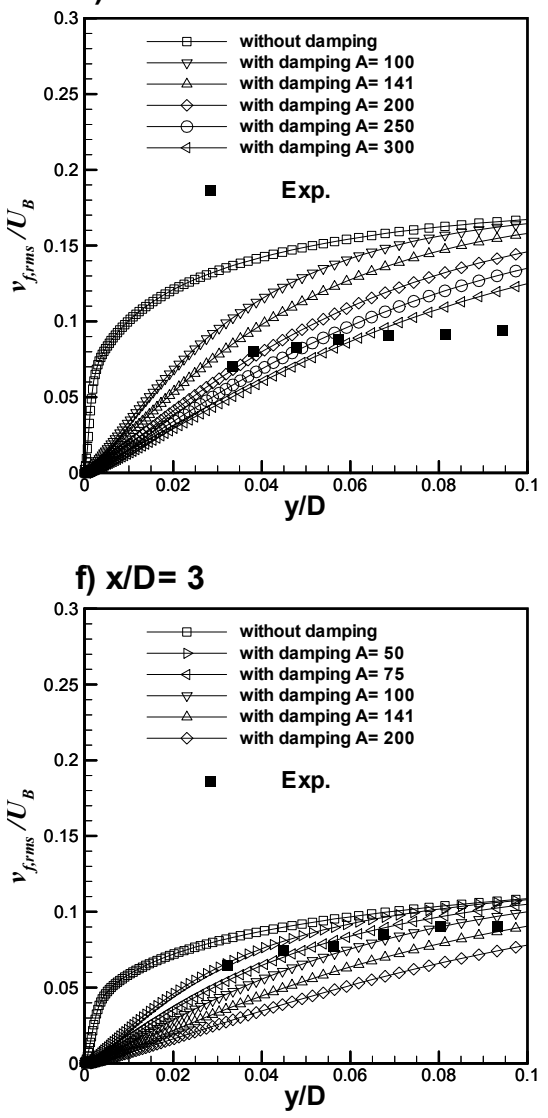

Figure 4.33. Calibrating normal-to-wall RMS fluctuation using SST model without Kato and Launder modification (a-c), and with Kato and Launder modification (df) for $L / D=6$ at three stations: $x / D=0.5,1.5$ and 3 . 
ough the marked correction. However, using the second procedure (best fit basis) the value of $A=200$ (diamond symbols) gives the best fit to the experimental data (Cooper et al., 1993) overall the stations (with and without Kato and Launder modification) despite the excessive damping at $x / D=3$, which has no effect on the deposition in this region for $L / D=6$.

Based on this critical evaluation, values of $A=100$ and 200 are chosen for $L / D=2$ and 6, respectively. For $L / D=4$, the value of $A=100$ is chosen since the turbulent features for $L / D=2$ and 4 , including the fluctuating velocities, are very similar (Cooper et al., 1993). These values were used in the correction function in order to obtain the corrected normal-to-wall RMS fluctuating velocities to predict the particle deposition in the impinging jet flow. Therefore for improved particle deposition prediction, the corrected normal-to-wall RMS fluctuating velocity can be expressed as

$w_{f, r m s, \text { corr }}^{\prime}=\left(1-e^{\frac{-y^{+}}{100}}\right)(2 k / 3)^{0.5}$

hence, the corrected kinetic energy can be written as

$k_{\text {corr }}=\left(1-e^{\frac{-y^{+}}{100}}\right)^{2} k$

By considering Equation (4.6) and using Equation (3.15), the corrected eddy lifetime can be written as

$\tau_{e}=0.2\left(1-e^{\frac{-y^{+}}{100}}\right)^{2}(k / \varepsilon)$

Equations (4.5 and 4.7) were used for the $L / D=2$ and 4 cases. Similarly, for the $L / D=6$ case, the following corrected normal-to-wall RMS fluctuating velocity 
$w_{f, r m s, \text { corr }}^{\prime}=\left(1-e^{\frac{-y^{+}}{200}}\right)(2 k / 3)^{0.5}$

and the eddy lifetime

$\tau_{e}=0.2\left(1-e^{\frac{-y^{+}}{200}}\right)^{2}(k / \varepsilon)$

were used. In the present study, the normal-to-wall RMS fluctuating velocity was corrected in regions that are in the buffer layer and the laminar sublayer near the wall (distant from the turbulent core). This correction (Equations 4.5-4.9) is implemented in the developed tracking code. The results are presented in the following paragraphs.

Figure 4.34 shows the deposition density curves as a function of $r / D$ for $L / D=2$ obtained with turbulent tracking and near-wall correction (i.e., Equations 4.5 and 4.7) at different $y^{+}$values. The predictions are computed using RANS SST without and with Kato and Launder modification along with modified EIM. As can be seen in Figure $4.34 \mathrm{a}$, the correction at $\mathrm{y}^{+} \leq 10$ has relatively close prediction to the experiment of Burwash et al. (2006) (dark square) at $r / D \geq 1.6$ despite the overprediction noted at $r / D \leq$ 1.6, which results due to the accumulation of particles in the low turbulence intensity region. However, it has better behavior over the turbulent tracking and the correction at $\mathrm{y}+\leq 5$, which does not show significant improvement over the turbulent tracking.

For computations using Kato and Launder modification as shown in Figure 4.34b, the turbulent tracking has a better prediction than the case without Kato and Launder modification although it still overpredicts the experiment at about $r / D \leq 1.8$. It can be noted that while the correction at $\mathrm{y}^{+} \leq 10$ (dot dash lines) leads to an underprediction over most of the $r / D$ range, the correction at $\mathrm{y}^{+} \leq 5$ (dash lines) shows significant 
improvement over the turbulent tracking. It can be noted that the correction at $y+\leq 5$ is able to capture the experimental peak accurately, and highly improves the deposition at the beginning of the wall jet region and close to the stagnation point. Also, it behaves closely as the experiment over the entire range of $r / D$ in spite of the small overpredictions downstream the wall jet and at $0.2<r / D<1$. This overprediction is likely attributable to

a)

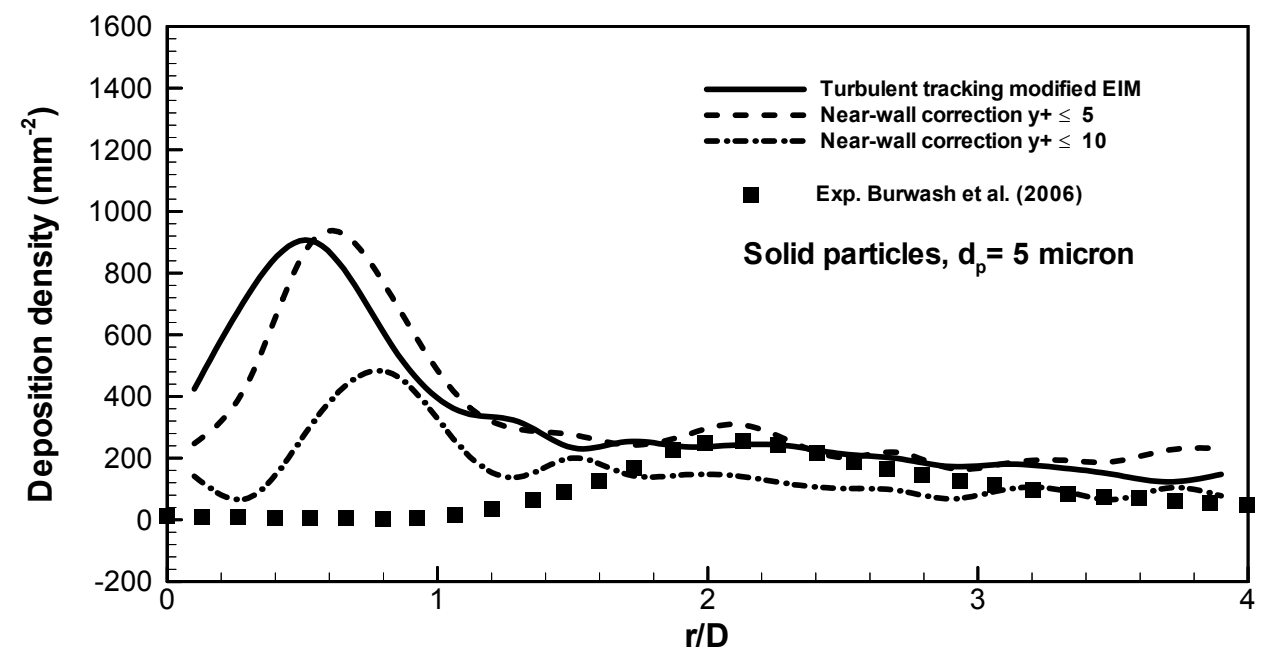

b)

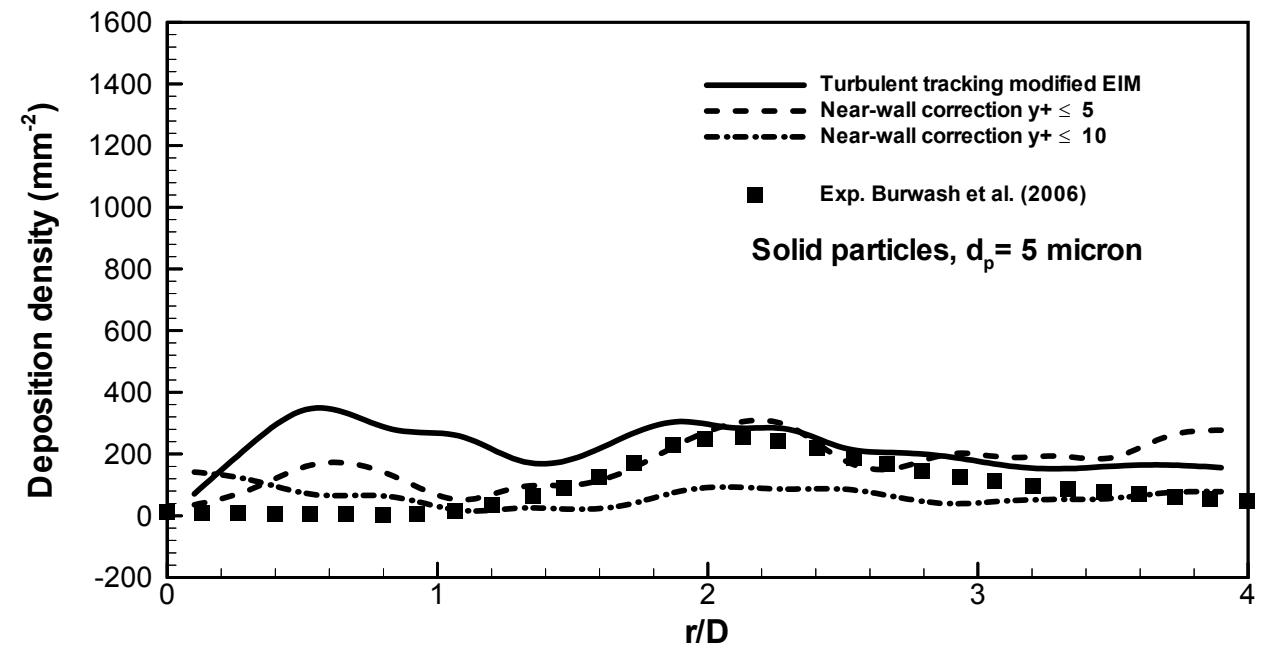

Figure 4.34. Deposition density for $\mathrm{L} / \mathrm{D}=\mathbf{2}$ from turbulent tracking and near-wall correction at different $y^{+}$using modified EIM and: (a) SST without Kato and Launder modification, and (b) SST with Kato and Launder modification. 
the particle accumulation effect that still persists, but at lesser extent than the case without Kato and Launder modification. The improved prediction for the deposition density is associated to the effective performance of the near-wall correction function used in the present analysis. The near-wall correction reduces the RMS normal-to-wall fluctuating velocity to an adequate level, especially in the wall jet region. As a result, it also reduces the excessive deposition.

Similarly for $L / D=4$, as shown in Figure 4.35 a, the deposition density that is predicted with correction at $\mathrm{y}^{+} \leq 10$ demonstrates significant improvement over the turbulent tracking, which leads to an overprediction over the $r / D$ range. This improvement is enhanced to the reduction in the RMS normal-to-wall fluctuating velocity due to the use of the near-wall correction function. It can be noted, although the small underprediction seen at $r / D<0.3$ that is caused by the underprediction of the RMS normal-to-wall fluctuating velocity, the deposition density at $\mathrm{y}^{+} \leq 10$ behaves closely as the experiment (Burwash et al., 2006) unlike the correction at $y^{+} \leq 15$ that leads to an underprediction over the $r / D$ range.

In Figure 4.35b (using Kato and Launder modification), although the turbulent tracking has relatively good prediction at $r / D<0.8$, it still overpredicts the experiment beyond $r / D=1$, i.e., within the wall jet region. This results due to the fact that Kato and Launder modification performs reasonably well very close to the stagnation point $(r / D<$ 0.4), but has no effect in the wall jet region where high values of the RMS normal-to-wall fluctuations remain and thus lead to high deposition. Using the near-wall correction at $y^{+}$ $\leq 10$ and 15 improves the prediction within this region (at $r / D \geq 1.2$ ) because of reducing 
a)

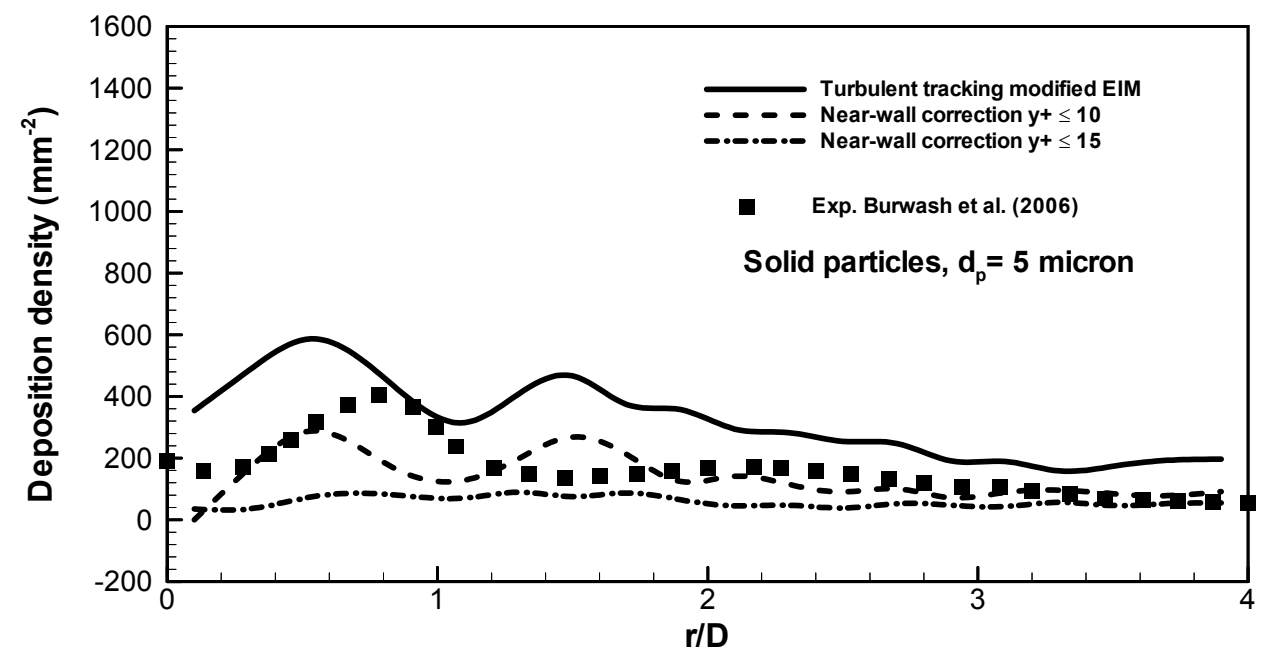

b)

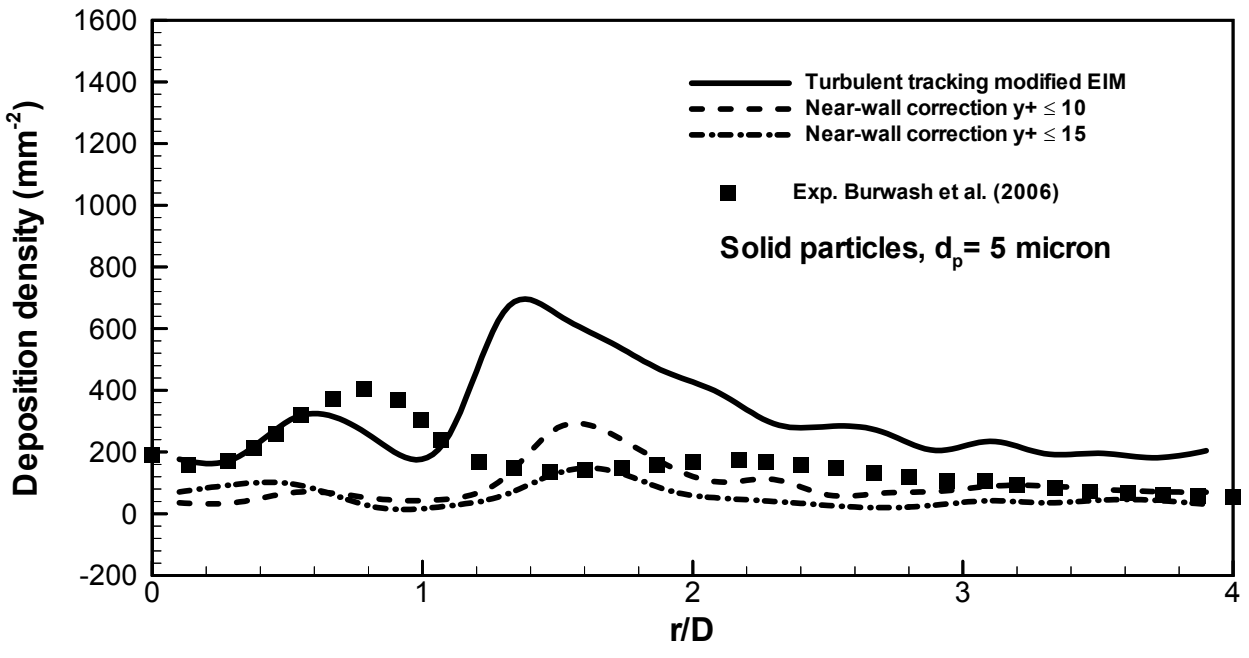

Figure 4.35. Deposition density for $\mathrm{L} / \mathrm{D}=\mathbf{4}$ from turbulent tracking and near-wall correction at different $y+$ using modified EIM and: (a) SST without Kato and Launder modification, and (b) SST with Kato and Launder modification.

the RMS normal-to-wall fluctuating velocity. However, the corrections at $y+\leq 10$ and 15 lead to an underprediction of the experimental peak at $0.4 \leq r / D \leq 1.1$ although they show a similar trend as the experiment close to the stagnation point at $r / D<0.4$. The underprediction, which is noted at the beginning of the wall jet $(0.4 \leq r / D \leq 1.1)$, is pro- 
bably because of the unavoidable reduction in the RMS normal-to-wall fluctuating velocity in this region.

From the experimental deposition data of Burwash et al. (2006), the deposition in cases $L / D=2$ and 4 occurs mainly in the wall jet region where the shear flow is dominant. In this region, the kinetic energy is highly produced by near-wall shear strains (region of strong streamline curvature). However, for $L / D=6$, the deposition behavior is highly affected by the presence of the stagnation point since the maximum deposition occurs very close to the stagnation point. As mentioned earlier, the impinging jet flow is complex due to the presence of the stagnation point where the kinetic energy production is dominated by normal strain. Indeed, it is known that very close to the stagnation point the friction velocity $u^{*}$ is very low (nearly zero) unlike in the wall jet region where it exhibits higher values. In addition, the turbulence kinetic energy (fluctuating velocities) that is produced near the stagnation point is less than the kinetic energy that is produced in the wall jet region downstream the stagnation point. Thus, applying the damping function in a region very close to the stagnation point affects the deposition and leads to a significant underprediction in the $L / D=6$ case. Therefore, due to the lack of data for the normal-to-wall RMS fluctuating velocity close to the stagnation point, and to improve the deposition prediction, the application of the correction using Equations (4.8 and 4.9) is adjusted such that the excessive effect of the damping can be reduced and the desired features in different regions can be obtained.

The adjustment of the application of the correction is based on a regional correction where the damping function switches between the stagnation region (very close to the 
stagnation point) and the wall jet region. Away from the stagnation point, i.e., at the beginning of the wall jet region and farther downstream, the function uses higher values of $y+$ (large correction domain). As the stagnation point is approached, the function uses lower values of $\mathrm{y}^{+}$(smaller correction domain). The regions at which the switch takes place are designated by critical distances of $r_{\mathrm{rr}, 1} / D=0.2$ and $r_{\mathrm{cr}, 2} / D=0.8$. At those two distances, the trend of the experimental deposition curve (Burwash et al., 2006) exhibits some variations, changing as the stagnation point is approached. In this adjustment, two correction domains of $y_{c r, 1}^{+}$and $y_{c r, 2}^{+}$were used. The values of $y_{c r, 1}^{+}$and $y_{c r, 2}^{+}$were adjusted to obtain the best agreement between the predictions and the experiment. The value of $y_{c r, 1}^{+}$is always smaller than $y_{c r, 2}^{+}$. Using this adjustment, the effect of extra damping very close to the stagnation point can be eased.

Figure 4.36 shows the deposition density curves obtained with the turbulent tracking and using the adjustment of the application of near-wall correction of Equations (4.8 and 4.9) for $L / D=6$. The predictions are computed without and with Kato and Launder modification. The correction is carried out using two different sets of $y_{c r}^{+}$, i.e., set1 is $y_{c r, 1}^{+} \leq 2$ and $y_{c r, 2}^{+} \leq 10$ and set 2 is $y_{c r, 1}^{+} \leq 2$ and $y_{c r, 2}^{+} \leq 12$. As can be seen from Figure 4.36a, as the adjustment of the near-wall correction is used, the predicted deposition density is significantly reduced, giving an improved behavior compared to turbulent tracking, especially very close to the stagnation point. It can be noted that the correction with $\mathrm{y}_{\mathrm{cr}, 1}^{+} \leq 2$ and $\mathrm{y}_{\mathrm{cr}, 2}^{+} \leq 12$ yields the closest prediction to the experiment of Burwash et al. (2006), although the slight overprediction noted at approximately $0.7 \leq$ 
$r / D \leq 1$, which might be due to the excessive kinetic energy that still remains in spite of the applied near-wall corrections.
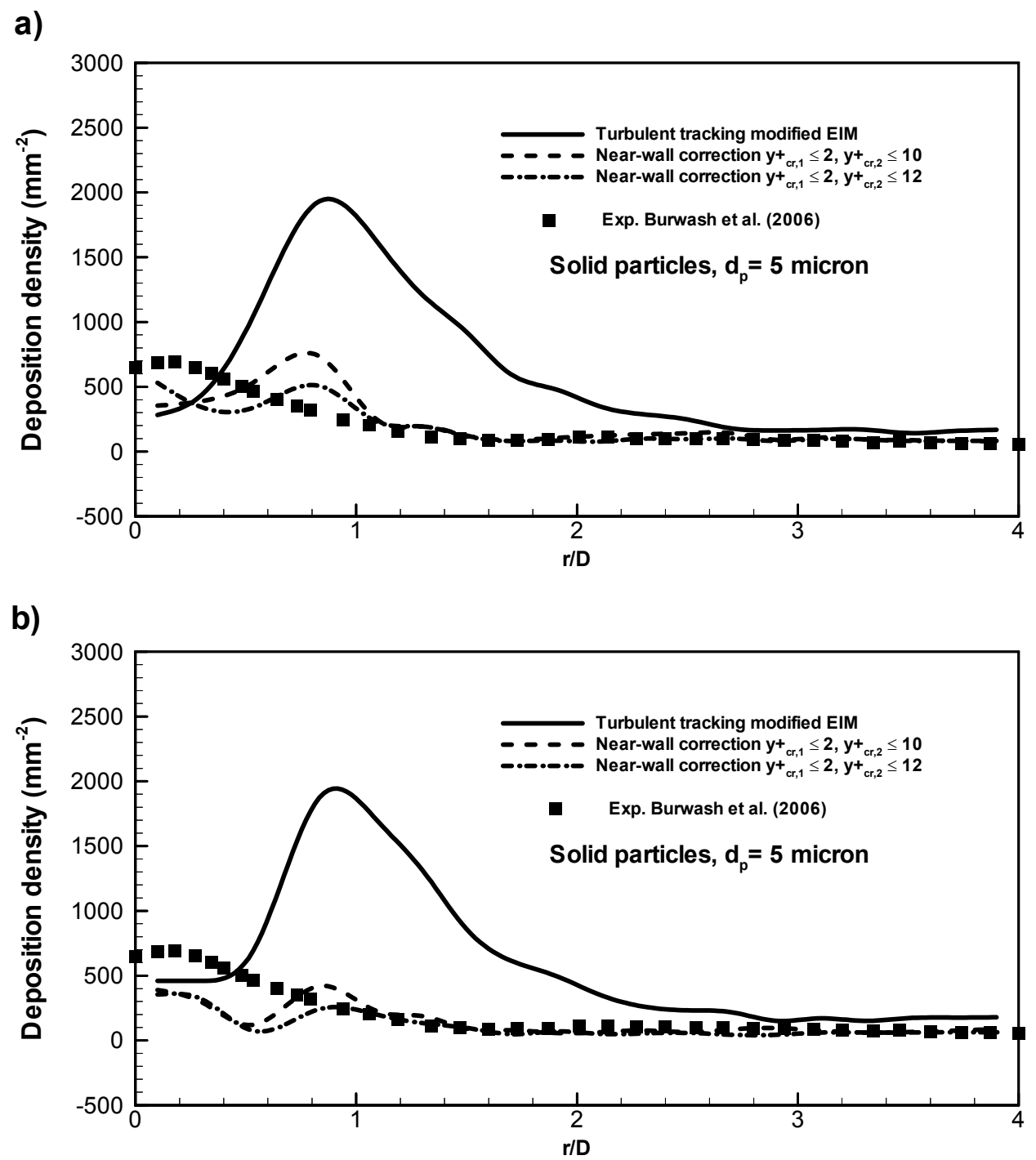

Figure 4.36. Deposition density for $L / D=6$ from turbulent tracking and near-wall correction at different $y+$ using modified EIM and: (a) SST without Kato and Launder modification, and (b) SST with Kato and Launder modification.

In Figure 4.36b (using Kato and Launder modification), the turbulent tracking has a similar behavior as in without Kato and Launder modification, although it yields an improved prediction very close to the stagnation point. When the adjustment of the near- 
wall correction is used for the two sets of $\mathrm{y}_{\mathrm{cr}}^{+}$, the deposition is significantly improved and agrees with the experiment at approximately $r / D \geq 0.7$. At approximately $r / D<0.7$, the deposition with the two sets of $\mathrm{y}_{\mathrm{cr}}^{+}$has a similar trend as the experiment, although the small underprediction very close to the stagnation point that might result due to the extra damping effect for the RMS normal-to-wall fluctuating velocity despite using small correction domains.

The differences that appear between the simulation results and the experimental data can be attributed to the effect of the accumulation of the particles in certain regions, in spite of allowing the eddy velocity scale to vary during the eddy-particle interaction in the modified EIM. This is more sensible for the $L / D=2$ case, and at lesser degree for the $L / D=4$ case. In the $L / D=2$ case, since the maximum kinetic energy takes place in the middle of the wall jet region $(1.5 \lesssim x / D \lesssim 2.3)$, as shown in the contour Figure $4.26 \mathrm{~d}$, and as the particle reaches this region very close to the wall, it receives a larger turbulence intensity and thus moves within the eddy to a region of low turbulence intensity. The particle continues to move within the eddy, until the eddy lifetime ends and another eddy forms with a lower velocity scale such that the particle is unable to return back to the high turbulence intensity region. Therefore, this leads to the accumulation of the particles in low turbulence region around $0.2 \leq r / D \leq 1.4$, and thus causes the peak (seen in Figure 4.34a) despite applying the near-wall correction. This accumulation of the particles in the low turbulence intensity region near the wall does not affect the particle concentration because the volume fraction of the particles is very low. The volume fraction considered in the present research is $\Phi_{\mathrm{p}}=10^{-9}$, which is within the range $\left(\Phi_{\mathrm{p}} \leq\right.$ 
$10^{-6}$ ) in which the particles have negligible influence on the fluid phase (Elghobashi, 1994). Thus, the one-way coupling interaction is not affected.

One of the causes, which might lead to a small difference between the simulation and the experimental data, is the hypothetical presence of the particle bouncing effect in the experiment (Burwash et al., 2006), especially for $L / D=2$. Due to the short nozzle-tosurface distance, the particles as they touch the wall, bounce off, and eventually redeposit at farther distance from the stagnation point. However, the effect of bouncing and reentrainment is assumed to be very small due to the small Stokes number used in the present research. This effect could be significant for larger Stokes numbers. Several researchers showed that heavy particles (high Stokes numbers) bounce off and reentrained to the impinging flow after impacting the impingement wall. In their investigated Stokes number range (0.6-2.4), Anderson and Longmire (1995) noted that the large Stokes number particles bounce off to greater heights above the impingement wall unlike the small Stokes number particles, which remain close to the wall. As a result, this bouncing affects the particle behavior to follow the fluid phase especially for larger particle sizes.

Another reason for the differences between the present simulation results and the experiment is the calibration against a high Reynolds number data. The calibrated nearwall correction function is based on the experiment of Cooper et al. (1993) that used higher Reynolds numbers $(\operatorname{Re}=23000$ for $L / D=2$ and $\operatorname{Re}=70000$ for $L / D=6)$ than the experiment of Burwash et al. (2006). Therefore, as the function is used to describe the normal-to-wall fluctuation behavior for the deposition analysis (based on Burwash's et al 
experiment), this is probably partly responsible for the difference between the present simulation and the experiment (Burwash et al., 2006). Also, part of this difference, which appears to affect the deposition for all $L / D$ cases, comes from the limitations of the fluid flow solution (single-phase flow) that results due to the imbalance of the production of turbulence kinetic energy near the stagnation point and in the wall jet region in spite of the inclusion of the shear stress limiters in the SST turbulence model.

\subsection{Conclusion}

This chapter presented the deposition results obtained using RANS SST/EIM for the three nozzle-to-surface distances of $L / D=2,4$ and 6 . The particle deposition density obtained with the turbulent tracking "without correction", using classical and modified EIM, demonstrates higher predictions than the experimental data for all the $L / D$ cases. Also, the results have shown that the nozzle-to-surface distance effect is significant for the particle deposition. In shorter nozzle-to-surface distance $(L / D=2)$, the particles deposit around the stagnation point forming annular area (ring-like pattern) as the experiment. This annular area moves towards the center (stagnation point) as the nozzleto-surface distance is increased as shown in Figures 4.22j, 4.22k and 4.221.

The present analysis has shown that the proper modeling of the near-wall normalto-wall fluctuating velocity is important for accurate prediction of the particle deposition. Therefore, the near-wall correction function calibrated based on the impinging jet flow characteristics was adopted to give an adequate representation of the normal-to-wall fluctuation. By employing this near-wall correction, the deposition density has an overall better level of agreement with the experimental data. By comparing Figures 4.34, 4.35 and 4.36 with Figures 4.17-4.19, it can be deduced that using the near-wall correction, 
which accounts for the near-wall features close to the impingement wall, leads to an improved prediction when compared with the turbulent tracking that was obtained using both classical EIM (CFX code) and modified EIM (tracking code), and the eddy ratio correction. Therefore, the near-wall correction has a better performance in predicting the particle deposition in the impinging jet flow than the eddy ratio correction that was presented in Subsection 4.6.2. 


\section{Chapter 5: Results for RANS-RSM with EIM}

In this chapter, the results from numerical simulations using another approach, RANS RSM-BSL/modified EIM, where the Reynolds stresses are obtained individually, are presented. In the present analysis, the modified EIM, which was adopted in the in-house tracking code that is extended in order to account for the RSM model features, was used to predict the deposition density by "turbulent tracking" (without corrections). The tracking code in CFX is based on the isotropic lifetime and velocity scales of the eddies; therefore, it is inapplicable when RSM model is used. The near-wall correction function (Van Driest, 1956), based on the impinging jet flow characteristics that are predicted by RANS RSM-BSL, is also presented. The results using near-wall correction, which was incorporated in the tracking code and implemented through the modified EIM, are presented and compared with the results obtained by the turbulent tracking. The accuracy of the impinging jet flow field simulations that are conducted under the experimental conditions of Burwash et al. (2006) and Cooper et al. (1993) is validated by comparing with the experiment of Cooper et al. (1993) and the simulation of Craft et al. (1993). A comparison of the deposition results between RANS SST/EIM and RANS RSM$\mathrm{BSL} / \mathrm{EIM}$ is presented. Other effects, such as effect of an isotropic eddy lifetime, are reported and presented.

\subsection{Computational domain and numerical parameters}

For RANS RSM calculations, the same computational domains based on Burwash et al. (2006) experiment were used to perform particle simulations. These computational domains for $L / D=2,4$ and 6 cases and the choice of the $L / D$ values are described in 
Section 4.1. Furthermore, the same computational domains based on Cooper et al. (1993) experiment for $L / D=2$ and 6 were used to perform fluid flow simulations (single-phase) and thus calibrate the near-wall correction function. The numerical simulations of the fluid flow phase using RANS RSM-BSL were carried out with CFX 12.0 (Ansys, Inc.). The simulations using RANS RSM-BSL were initialized from a converged RANS SST velocity field. This is because RANS RSM-BSL (and RSM models in general) simulations are computationally more demanding than RANS SST, since an equation for each Reynolds stress is solved individually.

Flow parameters and conditions are the same as those introduced in Section 4.1. The particle simulations were conducted using the modified EIM that was adopted in the in-house tracking code, where the particle simulation conditions are the same as those in Subsection 3.3.1.3.

\subsection{Fluid flow}

Using RANS RSM-BSL that solves directly for the Reynolds stresses provides another representation of the flow field. Therefore, it is useful to give insight to the impinging jet flow field before presenting the particle deposition results. The profiles of the mean velocity magnitude and the RMS fluctuating velocity components along lines perpendicular to the impingement wall versus $z / D$ for $L / D=2$ are shown in Figures 5.1 and 5.2, respectively. As seen in Figures 5.1a-5.1c, the simulated magnitude profiles of the mean velocity (lines with open squares) have similar trends as in the experiment of Cooper et al. (1993) (dark squares). At $x / D=0.5$, the mean velocity profile is pressure dominated and, therefore, it has higher values at larger $z / D$ values, i.e., RMS fluctuations 

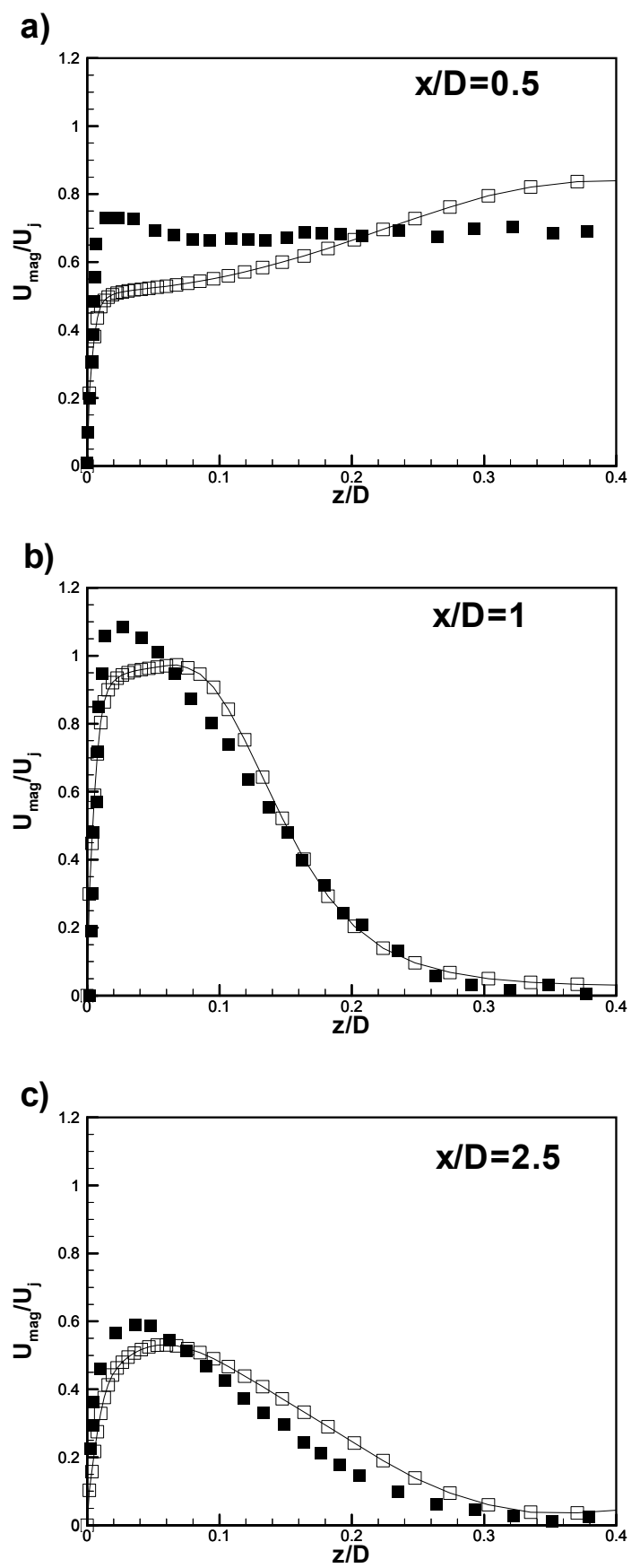

Figure 5.1. Mean velocity magnitude using RANS RSM-BSL model for $L / D=2$ at three stations: (a) $\mathrm{x} / \mathrm{D}=0.5$, (b) $\mathrm{x} / \mathrm{D}=1$, and (c) $\mathrm{x} / \mathrm{D}=2.5$. 
a)

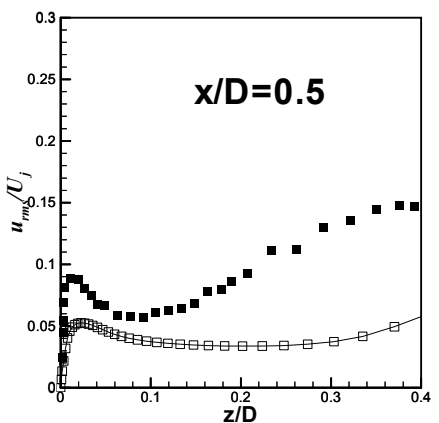

b)

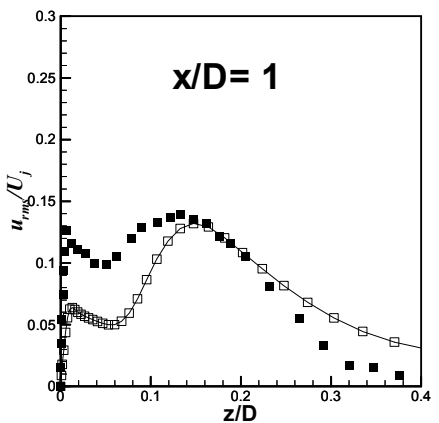

c)

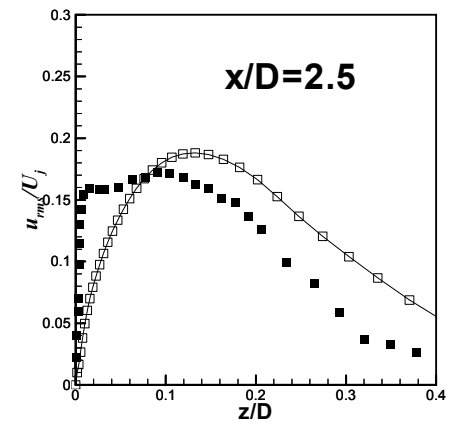

d)

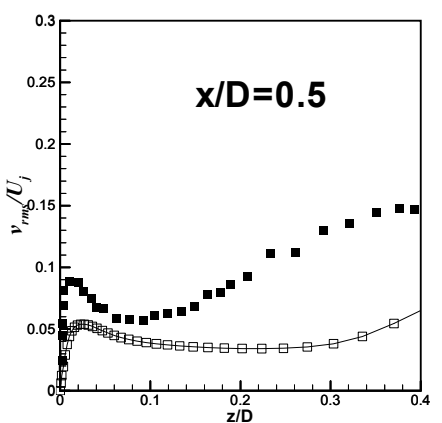

e)

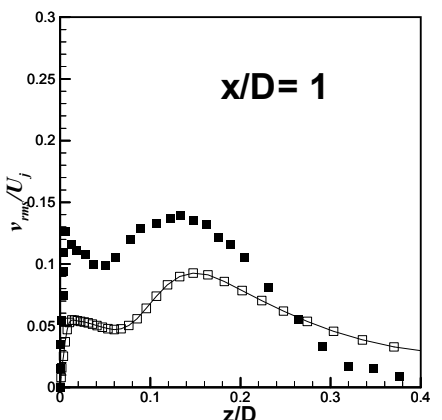

f)

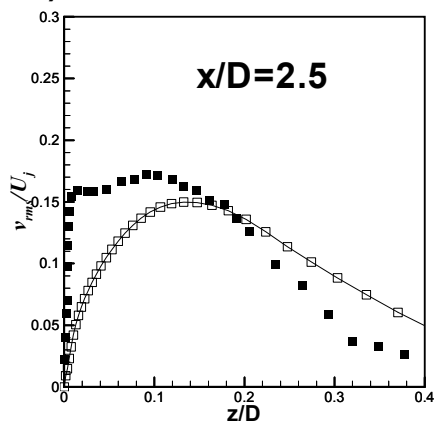

g)

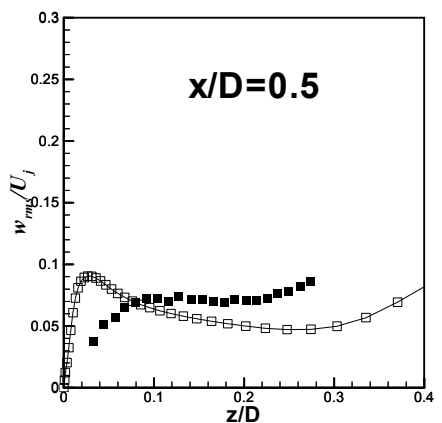

h)

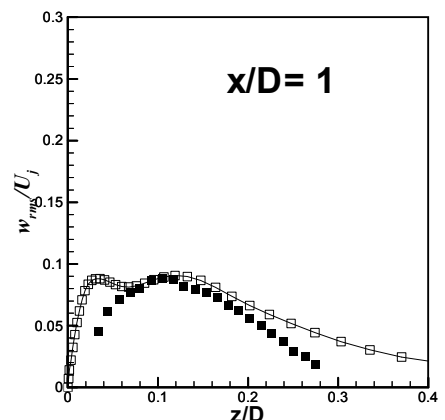

i)

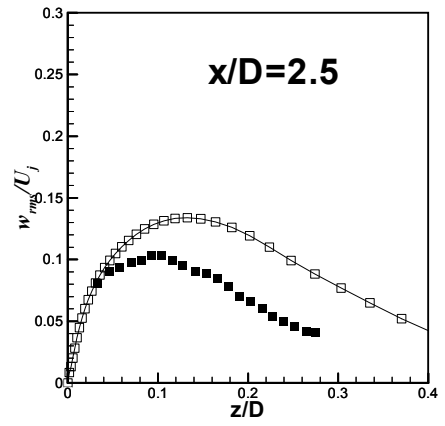

Figure 5.2. RMS fluctuating components using RANS RSM-BSL model for $L / D=2$ at three stations: $\mathrm{x} / \mathrm{D}=0.5,1$ and 2.5 .

do not affect its behavior significantly. However, as one moves downstream the wall jet region, especially at $x / D=1$ and 2.5 , the velocity magnitude increases sharply from a value of zero to some maximum due to the shear flow as the wall jet accelerates and subsequently decays to a very small value as the shear weakens at large $z / D$ values. It can be noted that although the simulated mean profiles are slightly underestimated, especially at $x / D=1$ and 2.5 , when compared with the experimental peaks close to the 
wall, they have similar decay rate as the experiment in the viscous sublayer region (i.e., at $z / D \leq 0.01)$. Moreover, it can be noted that the spreading of the mean velocity into the freestream is qualitatively similar to the experiment.

Figures 5.2a-5.2i show that the transverse $u_{r m s} / U_{j}$, spanwise $v_{r m s} / U_{j}$ and the normalto-wall $w_{r m s} / U_{j}$ RMS fluctuating velocity components (lines with open squares) have similar trends as the experiment of Cooper et al. (1993). At $x / D=0.5$, the fluctuating velocity trends increase smoothly to some peak close to the wall due to the flow acceleration (Cooper et al., 1993) before it decays sharply because of the wall effect. In the downstream at $x / D=1$, the fluctuating velocity profiles exhibit a gradual increase towards the wall (larger $z / D$ ) followed by a slight decrease due to the turbulent mixing. Then, they show a slight increase because of the shear induced by the flow's acceleration after which they decay under the wall effect. Similarly at $x / D=2.5$, the fluctuating profiles, generally, exhibit an increase towards the wall because of the shear that is produced in this region. Then, they show a nearly abrupt decay due to the wall effect. Although the underprediction noted in $u_{r m s} / U_{j}$ and $v_{r m s} / U_{j}$ at $x / D=1$ and, to a lesser extent, at $x / D=0.5$ as the impingement wall is approached, the fluctuations still reproduce the qualitative features. While the RMS normal-to-wall fluctuating velocity $w_{r m s} / U_{j}$ is overpredicted when compared to the experiment close to the impingement wall (at about $z / D<0.1$ ), it is reasonably predicted at larger values of $z / D$ although the slight overprediction noted at $x / D=2.5$. The overprediction in the RMS normal-to-wall fluctuating velocity component might be attributed to the fact of excessive predicted levels of shear strain, which replace the normal straining in the generation of turbulence energy. 
Similarly for $L / D=6$, the profiles of the mean velocity magnitude and the RMS fluctuating velocity components (lines with open squares) are shown in Figure 5.3 and 5.4, respectively. As shown in Figure 5.3, the simulated mean velocity profiles have similar trends as in the experiment (dark squares) despite the underprediction of the experimental peaks very close to the impingement wall. While the mean velocity profiles at $x / D=0.5$ and 1.5 have similar spreading as the experiment, except at $x / D=3$ that is slightly overpredicted, they also have similar decay rate as the experiment very close to the wall. The behaviors of the mean velocity trends are qualitatively similar to those described in the $L / D=2$ case. At $x / D=0.5$, the mean velocity is affected by the pressure and thus has different trend from the profiles at $x / D=1.5$ and 3 in which the RMS fluctuations affect their behaviors through the turbulence kinetic energy, which is produced during the wall jet development.

Figures 5.4a-5.4i show that the transverse $u_{r m s} / U_{j}$, spanwise $v_{r m s} / U_{j}$ and the normalto-wall $w_{r m s} / U_{j}$ RMS fluctuating velocity components, generally, have similar trends as the experiment although the underpredictions and overpredictions noted in their behaviors. At $x / D=0.5$, the fluctuations of $u_{r m s} / U_{j}$ and $v_{r m s} / U_{j}$ (in the experiment) exhibit smooth increase very close to the wall due to the induced shear by the flow's acceleration. Then, they exhibit a sharp decrease because of the wall effect. At $x / D=1.5$ and 3, the $u_{r m s} / U_{j}$ and $v_{r m s} / U_{j}$ fluctuations demonstrate a gradual increase as the wall is approached $(\mathrm{z} / \mathrm{D}<0.3)$ due to the shear produced in these regions, followed by a sharp decay at the wall. The $w_{r m s} / U_{j}$ fluctuations at $x / D=0.5,1.5$, and 3 show an increase, due to the shear produced during the wall jet development, before they decay gradually because of the 

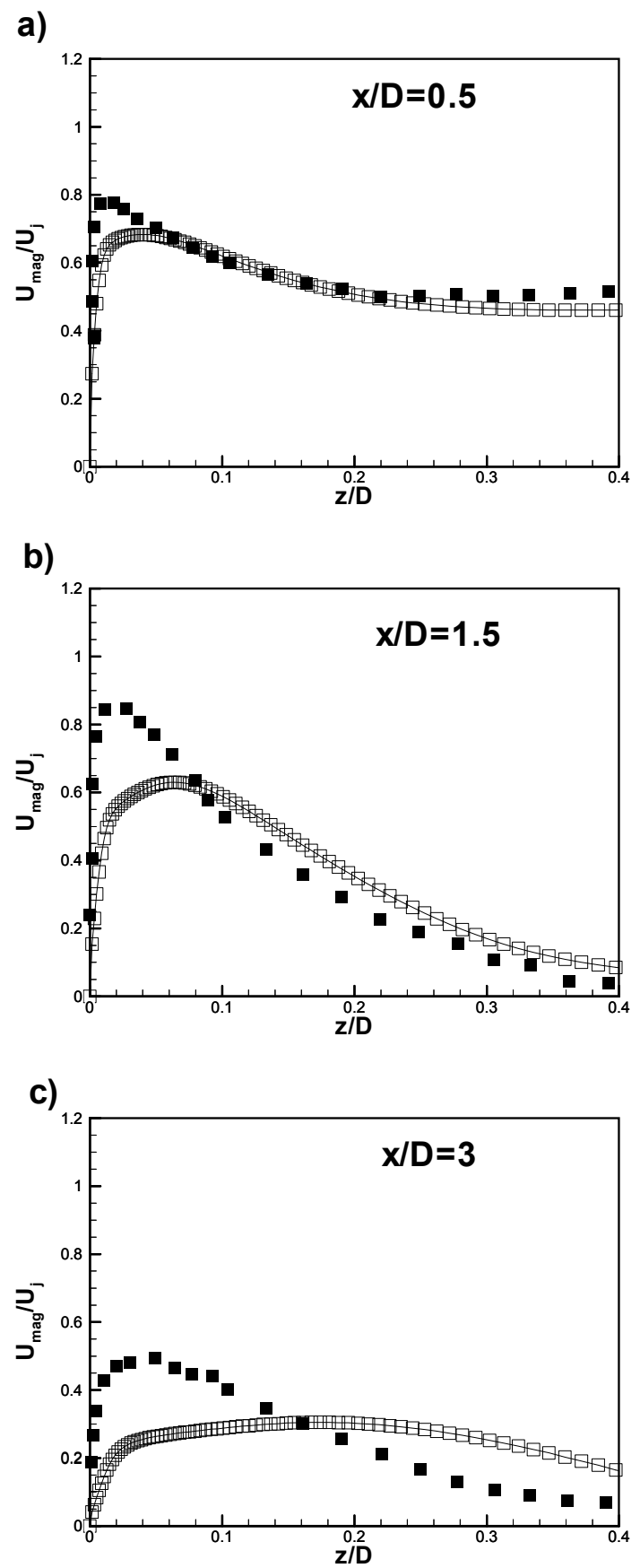

Figure 5.3. Mean velocity magnitude using RANS RSM-BSL model for $L / D=6$ at three stations: (a) $x / D=0.5$, (b) $x / D=1.5$, and (c) $x / D=3$. 
a)

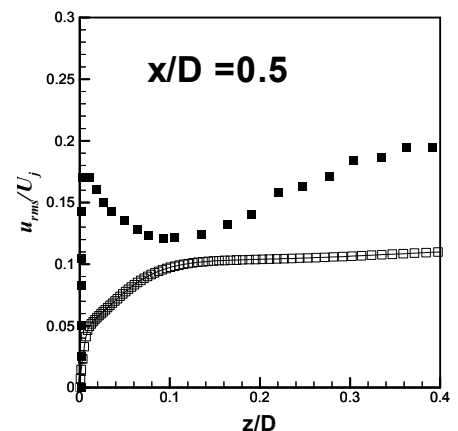

b)

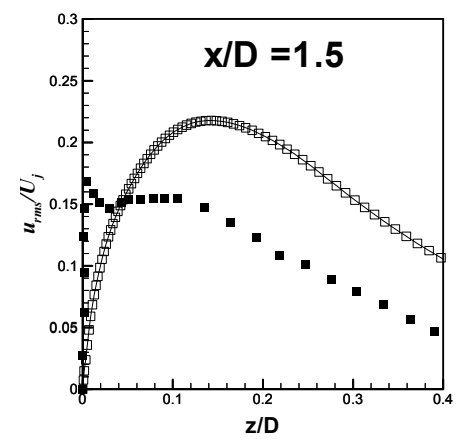

c)

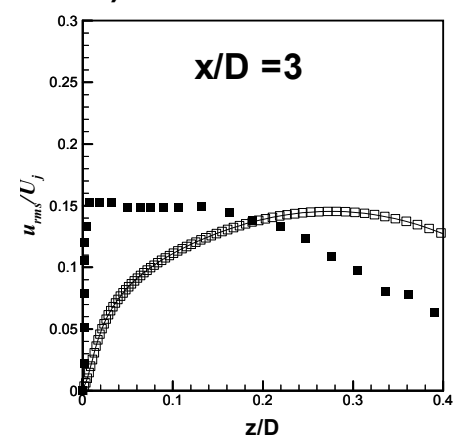

d)

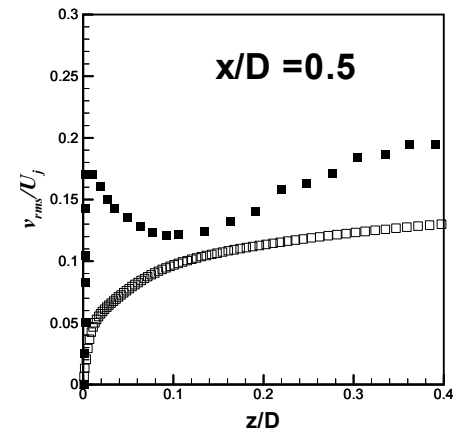

e)
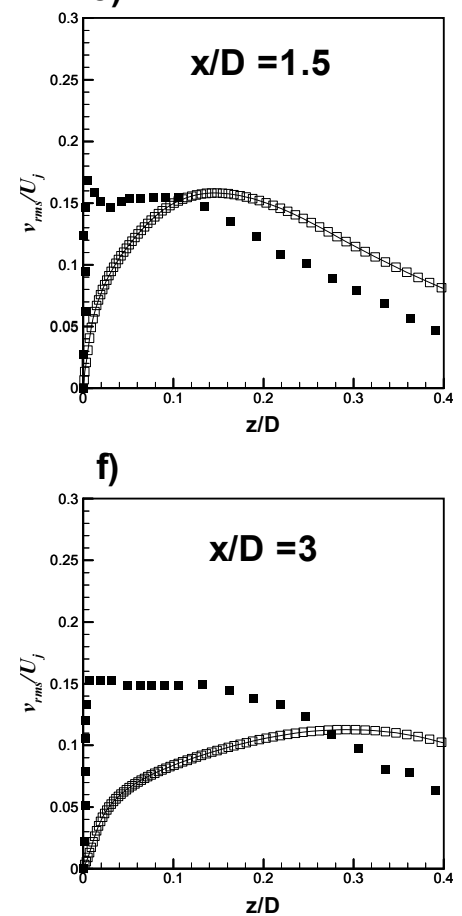

g)

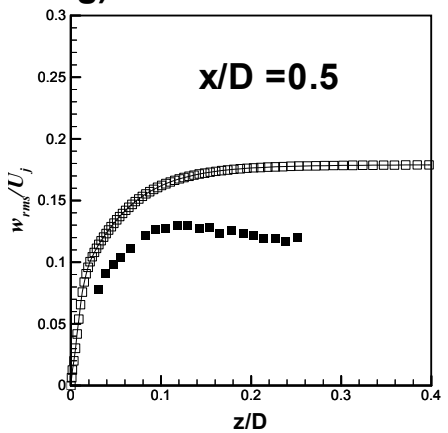

h)
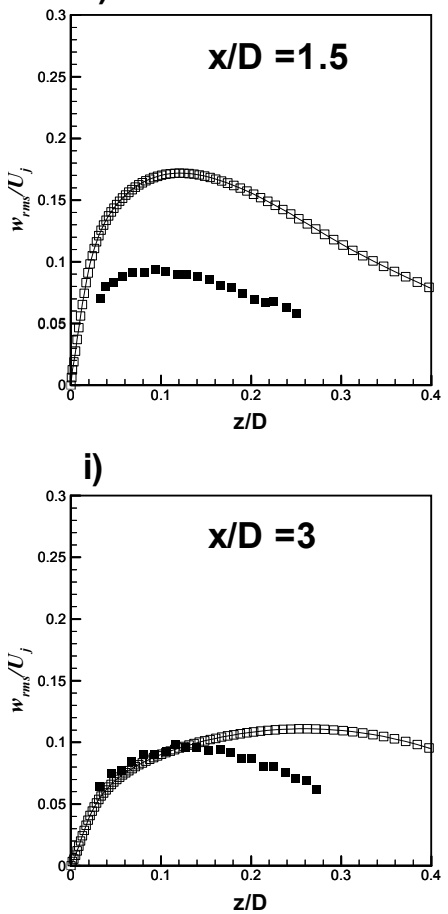

Figure 5.4. RMS fluctuating components using RANS RSM-BSL model for $L / D=6$ at three stations: $x / D=0.5,1.5$ and 3 .

wall effect. It can be noted that the RMS normal-to-wall fluctuating velocity $w_{r m s} / U_{j}$ at $x / D=0.5$ and 1.5 is overpredicted when compared to the experiment.

The differences between the simulation results and the experiment of Cooper et al. (1993) in Figures 5.1, 5.2, 5.3 and 5.4 are expected because of the differences in the flow parameters and inflow conditions between the present simulation, which is based on the 
experiment of Burwash et al. (2006), and the experiment of Cooper et al. (1993). In the present simulation, the Reynolds number is set to value similar to that of the experiment of Burwash et al. (2006), who reported only the deposition results. In the experiment, the Reynolds number is 10000 for all the nozzle-to-surface distance $L / D$ cases. However, in the experiment of Cooper et al. (1993), the Reynolds numbers used were 23000 and 70000 for $L / D=2$ and 6 , respectively. Also, the inflow condition has significant effect on the fluid flow. In the present simulation, which is performed under the conditions of the experiment of Burwash et al. (2006), the inflow velocity profile is nearly flat (Top-Hat profile), where the exit was a contraction nozzle. In contrast, in the experiment of Cooper et al. (1993), the flow exits from a pipe nozzle. Indeed, the present simulations are conducted in confined computational domains similar to the experiment of Burwash et al. (2006). However, the experiment of Cooper et al. (1993) has an unconfined set-up. The overprediction in the RMS normal-to-wall fluctuating component, which is shown for $L / D=2$ and 6 (Figures 5.2 and 5.4), can be attributed to the absence of the stress limiter in the RANS RSM-BSL model and the excessive predicted levels of shear strain. This may affect the prediction of mean flow as described in Section 4.5.

Although the RANS RSM-BSL model predicts the variations in the fluctuating velocities according to three space directions (anisotropy), it is unable to accurately predict the normal-to-wall fluctuating velocity near the impingement wall. The overprediction in the normal-to-wall fluctuating velocity component, which is the component responsible for the deposition, has consequences in predicting high deposition rates as will be shown in the next section. Therefore, for successful predictions of the 
particle deposition rate, it is necessary that the normal-to-wall fluctuating velocity be correctly modeled as will be shown later in this chapter.

\subsection{Deposition results using modified EIM}

\subsubsection{Turbulent tracking and mean flow tracking}

The simulated particle deposition density profiles, using turbulent tracking and mean flow tracking versus the radial distance from the stagnation point $r / D$, are shown in Figures 5.5, 5.6 and 5.7 for $L / D=2,4$ and 6 , respectively. These particle simulations were performed using the developed tracking code (modified EIM). The experimental data of Burwash et al. (2006) for $L / D=2,4$ and 6 are shown for comparison. As can be seen from the figures, the turbulent tracking (bold solid line) leads to a prediction qualitatively close to the experimental data, downstream of the wall jet region at $r / D \geq 2$, for all $L / D$ cases. However, the turbulent tracking significantly overpredicts the experimental data at region of $r / D<2$ for all $L / D$ cases. This significant overprediction of the particle deposition is not unexpected because of the high turbulence normal-to-wall fluctuating velocity, which highly affects the predicted deposition. Although the RSMBSL accounts for the anisotropy of turbulence near the wall, it predicts high turbulence fluctuating velocities and thus high kinetic energy as the wall jet starts to develop. Therefore, these results highlight the critical importance of the proper modeling of the RMS normal-to-wall fluctuating velocity, which plays significant role in particle deposition, in the near-wall region. Deposition using near-wall correction is discussed in the next section. 


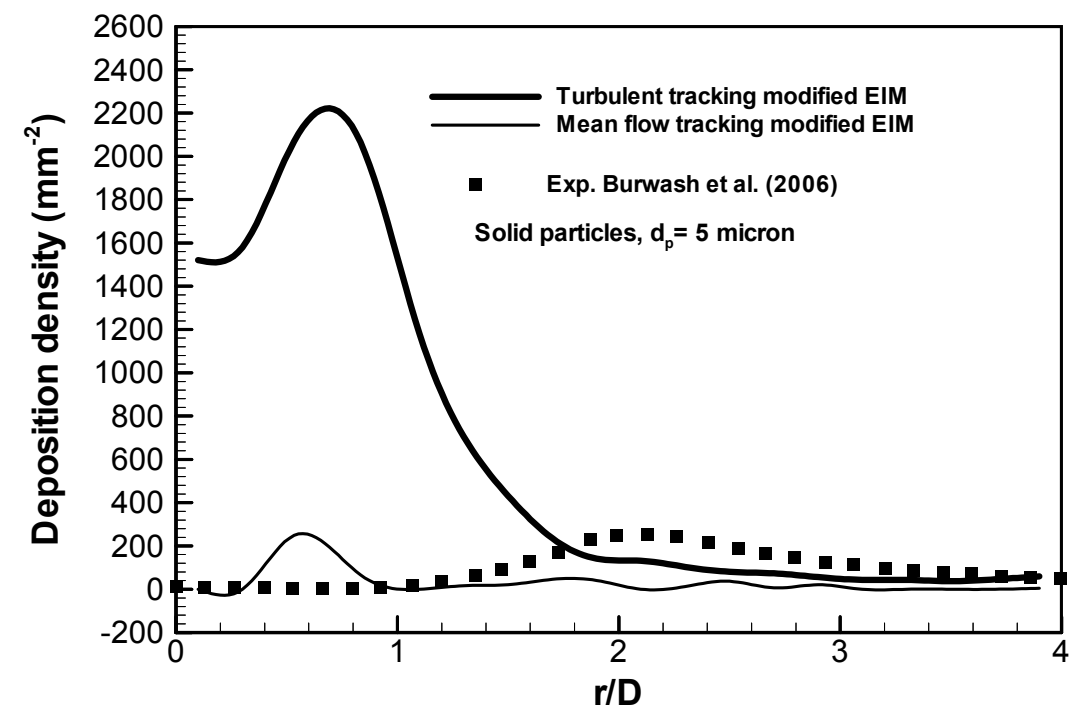

Figure 5.5. Deposition density from turbulent tracking and mean flow tracking for L/D = 2 using RSM-BSL/modified EIM.

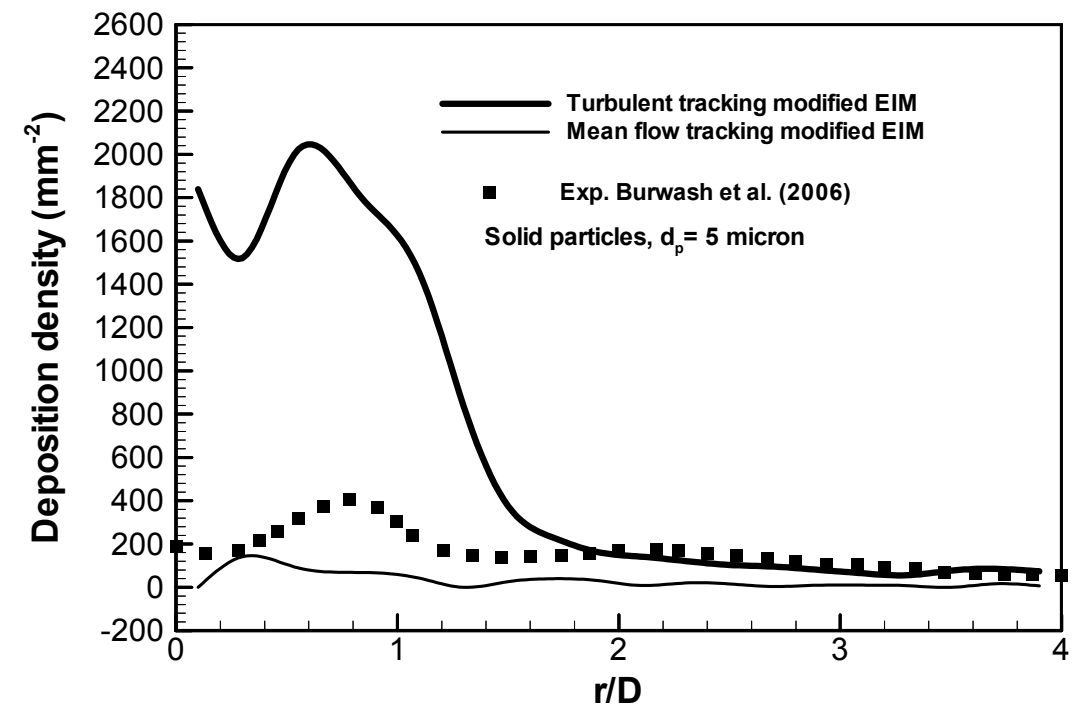

Figure 5.6. Deposition density from turbulent tracking and mean flow tracking for L/D = 4 using RSM-BSL/modified EIM. 


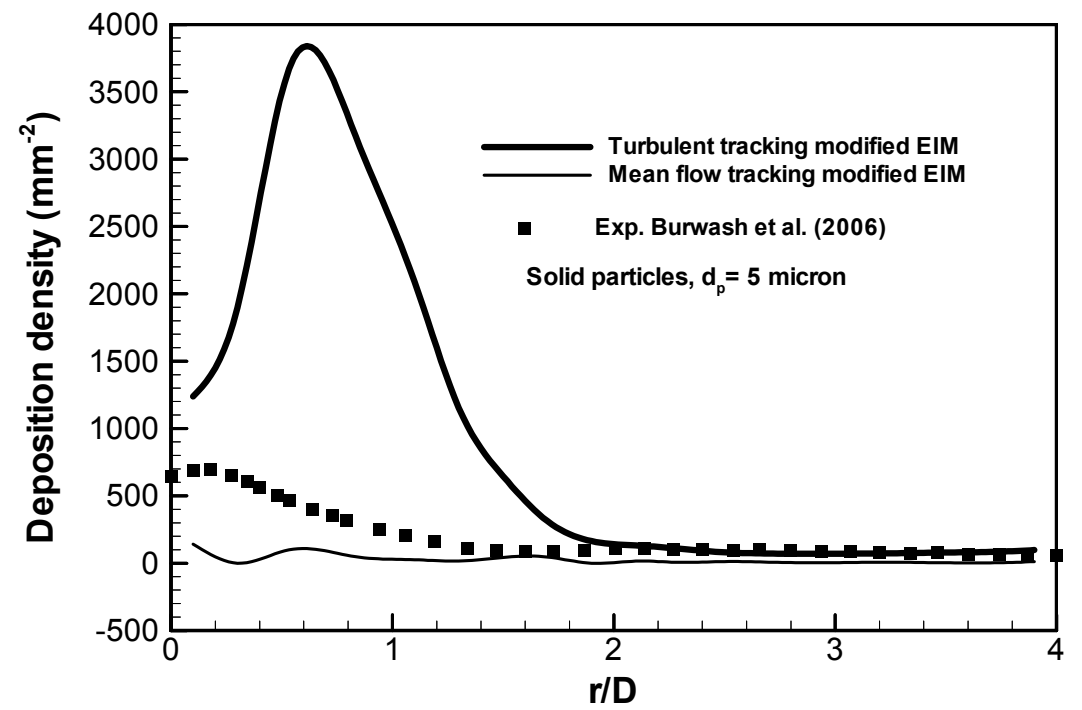

Figure 5.7. Deposition density from turbulent tracking and mean flow tracking for L/D = 6 using RSM-BSL/modified EIM.

The mean flow tracking (thin solid line), where the solid particles are deposited by means of inertial impaction mechanism, leads to an underprediction of the simulated deposition density for all $L / D$ cases compared to the experimental data of Burwash et al. (2006). It can be noted that the deposition density is almost nil downstream the wall jet region at $r / D>1$ for all $L / D$ cases. However, a few particles are deposited very close to the stagnation point in a different trend from the experimental data as shown for $L / D=2$. Similarly for $L / D=4$ (Figure 5.6) and 6 (Figure 5.7), few particles are deposited very close to the stagnation point and the simulated deposition density is lower than the experimental data, as expected.

By comparing the simulated deposition density results obtained by the turbulent tracking with those obtained by the mean flow tracking, significant difference exists. This means that these results indicate that the deposition of $5 \mu \mathrm{m}$ particles is strongly governed by turbulent dispersion (turbulent dispersion by fluctuating velocities) rather 
than by the inertial impaction, which is significant as the particles size increases. This is consistent with the results that were presented when the SST model with the classical and modified EIM was used.

\subsubsection{Near-wall correction}

As demonstrated in Section 5.2, although the RSM-BSL model accounts for the anisotropy in the near-wall region, it yields a high RMS fluctuating velocity in the normal-to-wall direction $w^{\prime} r m s$ as the wall is approached. As a result, this leads to an overprediction in deposition close to the stagnation point and at the beginning of the wall jet region when the turbulent tracking was used as presented in the preceding subsection. This is because the RANS RSM-BSL model in the CFX solver is formulated primarily for turbulent flows in the core region. Therefore, this necessitates the need for accurate modeling of the near-wall region such that the RMS normal-to-wall fluctuating velocity can be accounted for adequately for more realistic simulations.

The proposed function, introduced in Subsection 4.9.2 and based on Van Driest's (1956) damping factor, is utilized here to correct the RMS normal-to-wall fluctuating velocity that is obtained from RANS RSM-BSL model in order to obtain an accurate prediction of the particle deposition. Therefore, using the same procedure adopted earlier in Subsection 4.9.2.2, this function (Equation 4.3) should be calibrated by determining the value of $A$ in the function according to the impinging jet flow characteristics that are obtained from RANS RSM-BSL model. For this reason, series of numerical simulations for the primary flow (single-phase) using RSM-BSL model under conditions similar to 
those of the experiment of Cooper et al. (1993) were performed for nozzle-to-surface distances of $L / D=2$ and 6 .

As was shown in Subsection 4.9.2.2, the accurate representation of the RMS normal-to-wall fluctuating velocity is obtained by comparison between calculations and the experiment of Cooper et al. (1993). Therefore, this is carried out by comparing the RMS normal-to-wall fluctuating velocity, which is predicted by RSM-BSL model and corrected using Equation (4.3), with the experimental data at different stations. Using Equation (4.3), the corrected RMS normal-to-wall fluctuating velocity is obtained as

$v_{f, r m s, \text { corr }}^{\prime}=\left(1-e^{\frac{-y^{+}}{A}}\right)\left(\overline{v^{/ 2}}\right)^{0.5}$

As described earlier in Subsection 4.9.2.2, two different procedures (taking the average value and the best fit basis) were implemented to determine the value of $A$ in Equation (5.1). This is described in the following paragraphs.

The calibration of the RMS normal-to-wall fluctuating velocity using different values of $A$ is shown in Figures 5.8a-5.8c and 5.8d-5.8f for $L / D=2$ and 6 , respectively. As can be seen in the figure, the RMS normal-to-wall fluctuating velocity without damping (i.e., without correction) exhibits high levels as the impingement wall is approached. This causes an overprediction in the deposition when turbulent tracking is used. When the correction with Equation (5.1) is used in the near-wall region, the normal fluctuating velocity is lowered. For $L / D=2$, the average value (first procedure) of $A=$ 139 (gradient symbols) demonstrates higher levels of the RMS normal-to-wall fluctuating velocity compared to the experiment (dark square symbols). However, Using the second 
procedure leads to the value of $A=200$ (diamond symbols) that yields the closer fit to the experiment overall the stations $(x / D=0.5$ and 1$)$, which have the significant overprediction of the fluctuation, more than the value $(A=139)$ determined by the first procedure. Similarly for $L / D=6$, the average value of $A=243$ (gradient symbols) with the first procedure, although the close agreement with the experiment at $x / D=1.5$, it still has significant overprediction at $x / D=0.5$. Using the second procedure (best fit basis), the value of $A=300$ (diamond symbols) gives the best fit across most of the stations as shown in Figures 5.8d-5.8f. Therefore, from this analysis, it can be deduced that the best fit procedure leads to the best compromising value of $A$ across the near-wall region.

Based on this evaluation, values of $A=200$ and 300 were chosen for $L / D=2$ and 6 , respectively. For $L / D=4$, the value of $A=200$ was chosen since the turbulent features for $L / D=2$ and 4 , including the fluctuating velocities, are very similar (Cooper et al., 1993). These values were used in Equation (4.3) in order to obtain the corrected RMS normal-to-wall fluctuating velocity to predict the particle deposition in the impinging jet flow. Therefore, for improved particle deposition, the corrected RMS normal-to-wall fluctuating velocity

$w_{f, r m s, \text { corr }}^{\prime}=\left(1-e^{\frac{-y^{+}}{200}}\right)\left(\overline{w^{\prime 2}}\right)^{0.5}$ 
a) $x / D=0.5, L / D=2$

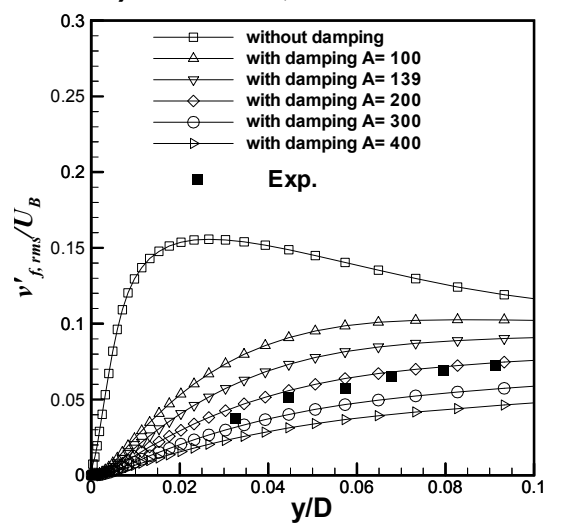

b) $x / D=1, L / D=2$

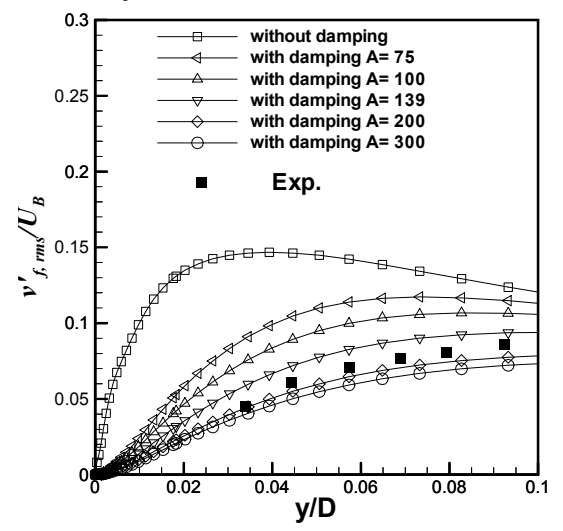

c) $x / D=2.5, L / D=2$

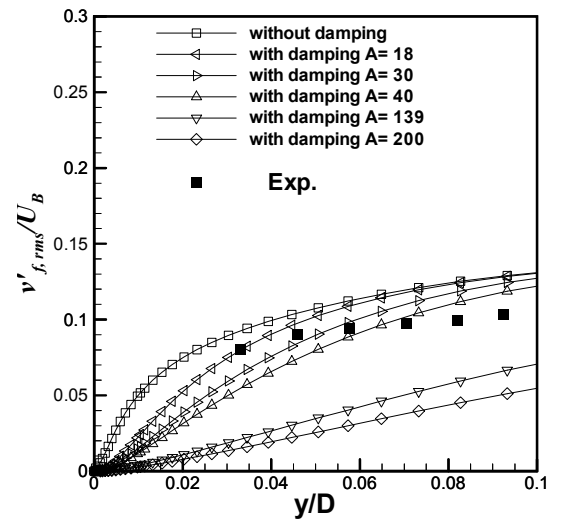

d) $x / D=0.5, L / D=6$

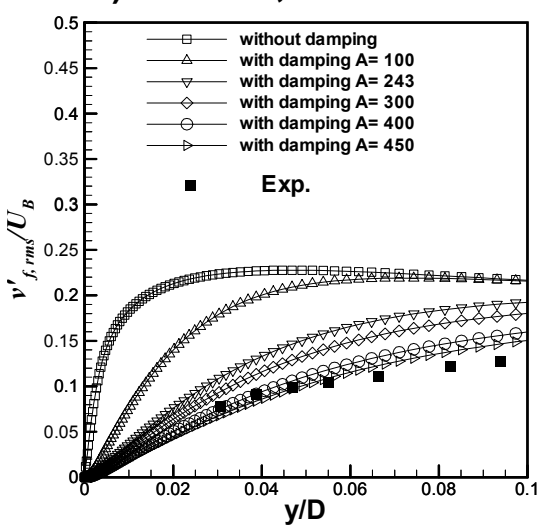

e) $x / D=1.5, L / D=6$
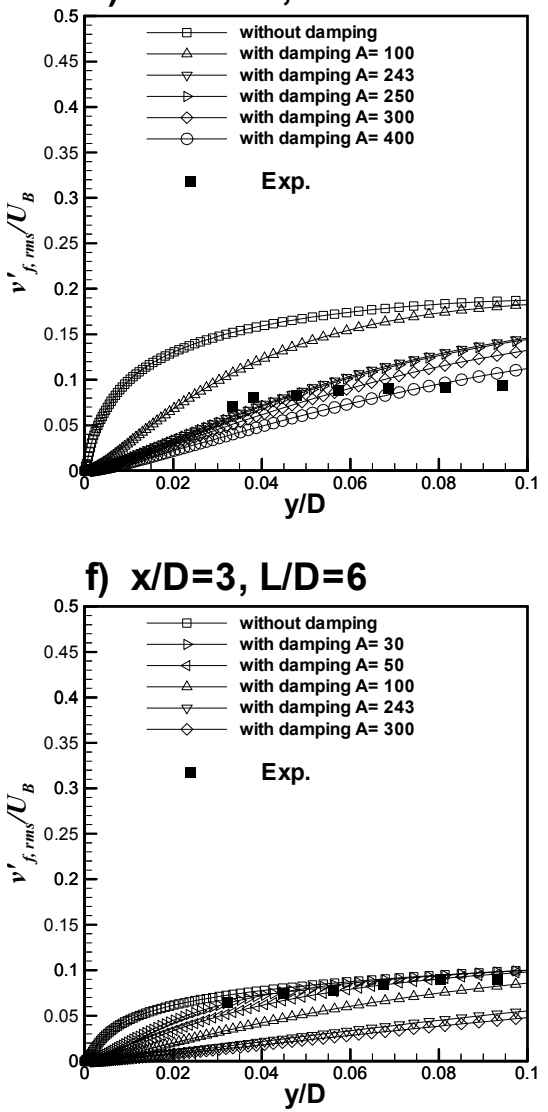

Figure 5.8. Calibrating RMS normal-to-wall fluctuation using RSM-BSL model for $L / D=2(a-c)$, and $L / D=6$ (d-f). 
and the corrected eddy lifetime

$$
\tau_{e}=2 C\left(\left(1-e^{\frac{-y^{+}}{200}}\right)\left(\overline{w^{\prime 2}}\right)^{0.5}\right)^{2} / \varepsilon
$$

were used for $L / D=2$ and 4 cases. Here, $C$ is equal to 0.1 . Also, the following corrected RMS normal-to-wall fluctuating velocity

$$
w_{f, r m s, \text { corr }}^{\prime}=\left(1-e^{\frac{-y^{+}}{300}}\right)\left(\overline{w^{\prime 2}}\right)^{0.5}
$$

and the corrected eddy lifetime

$$
\tau_{e}=2 C\left(\left(1-e^{\frac{-y^{+}}{300}}\right)\left(\overline{w^{\prime 2}}\right)^{0.5}\right)^{2} / \varepsilon
$$

were used for $\mathrm{L} / \mathrm{D}=6$ case. Here $C=0.1$.

In the present study, the RMS normal-to-wall fluctuating velocity was corrected in regions that are in the buffer layer and the laminar sublayer near the wall (distant from the turbulent core). This correction (Equations 5.2-5.5) is implemented in the developed tracking code. The results are presented in the following paragraphs.

Figure 5.9 shows the deposition density curves for $L / D=2$ obtained with turbulent tracking and near-wall correction (Equations 5.2 and 5.3). As the near-wall correction is used with corrections at $\mathrm{y}^{+} \leq 15$ and 20 , the predicted deposition density is clearly improved due to the effect of the near-wall correction in reducing RMS normal-to-wall fluctuating velocities. It can be noted that the correction at $\mathrm{y}+\leq 15$ has a similar trend as the experiment (dark square symbols) despite the slight underprediction at $r / D>1.8$. Also, it still shows a higher deposition than the experiment at about $0.5 \leq r / D \leq 1.6$ 
before it coincides with the experiment close to the stagnation point. This higher prediction in the deposition density can be attributed to the accumulation effect of the particles in the low turbulence intensity region, as discussed in Subsection 4.9.2.2, in spite of using RSM-BSL model and modified EIM with near-wall correction. The correction at $\mathrm{y}+\leq 20$ underpredicts the experiment more than the correction at $\mathrm{y}+\leq 15$ due to an excessive reduction in the RMS normal-to-wall fluctuating velocity component, although it shows a similar trend as the experiment at $r / D<1.6$.

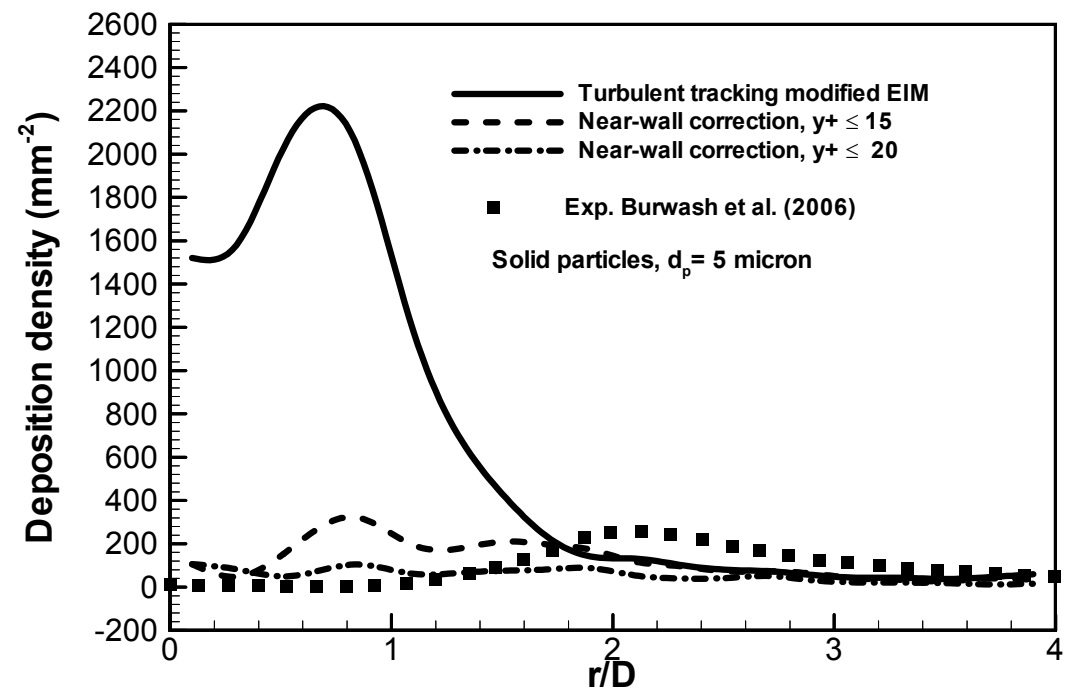

Figure 5.9. Deposition density for $L / D=2$ from turbulent tracking and near-wall correction with the new function at different $y+$ using RSM-BSL/modified EIM.

Similarly, Figure 5.10 shows the deposition density curves for $L / D=4$. As shown in the figure in comparison to the turbulent tracking, which shows a significant overprediction at $r / D<1.8$ compared to the experiment, the near-wall correction at $\mathrm{y}+\leq$ 10 and 12 highly improved the deposition density due to its performance in reducing the 
RMS normal-to-wall fluctuations. It can be noted that the correction at $\mathrm{y}+\leq 10$ yields good prediction farther downstream the wall jet region $(r / D>1.8)$ and, moreover, shows

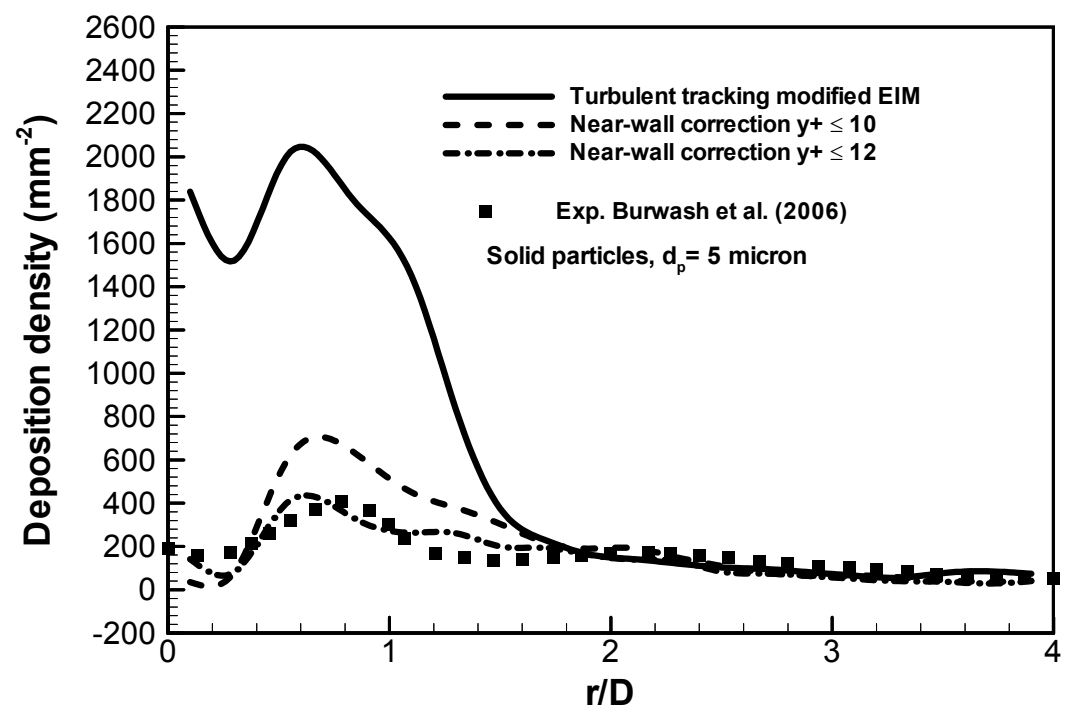

Figure 5.10. Deposition density for $\mathrm{L} / \mathrm{D}=4$ from turbulent tracking and near-wall correction with the new function at different $y+$ using RSM-BSL/modified EIM.

a similar trend as the experiment at the beginning of the wall jet region and close to the stagnation point $(r / D<1.8)$ although the slight overprediction of the experimental peak. This overprediction might be attributed to the presence of high RMS normal-to-wall velocity that causes high deposition density in this region. The correction at $y+\leq 12$ (dot dash line) yields the closest prediction to the experiment. It can be noted that this correction captures the experiment quite well over the entire range of $r / D$ despite the very small difference.

For $L / D=6$, the adjustment of the application of the correction, which is adopted in Subection 4.9.2.2, is implemented here using the near-wall correction (Equations 5.4-5.5) and the results are shown in Figure 5.11. Figure 5.11 shows the deposition density curves 
obtained with the turbulent tracking and the near-wall correction using two different sets of $\mathrm{y}_{\mathrm{cr}}^{+}$, i.e., set1 is $\mathrm{y}_{\mathrm{cr}, 1}^{+} \leq 4$ and $\mathrm{y}_{\mathrm{cr}, 2}^{+} \leq 20$ and set2 is $\mathrm{y}_{\mathrm{cr}, 1}^{+} \leq 4$ and $\mathrm{y}_{\mathrm{cr}, 2}^{+} \leq 25$. Unlike the turbulent tracking, the near-wall correction highly improved the deposition density at the beginning of the wall jet region and near the stagnation point due to its ability in reducing high values of the RMS normal-to-wall fluctuating velocities in the wall proximity. The correction with set1 (dash lines) still shows a slightly higher peak at the beginning of the wall jet (i.e., at $r / D \approx 0.8$ ) and a slightly higher deposition very close to the stagnation point, which is likely attributable to the remaining high RMS normal-towall fluctuating velocities. However, the correction with set2 (dot dash lines) leads to significant improvement and captures the experimental peak close to the stagnation point. Also, although the small underprediction at approximately $0.4 \leq r / D \leq 0.7$, it can be noted that the deposition density curve with this correction is in agreement with the experiment over the entire $r / D$ range.

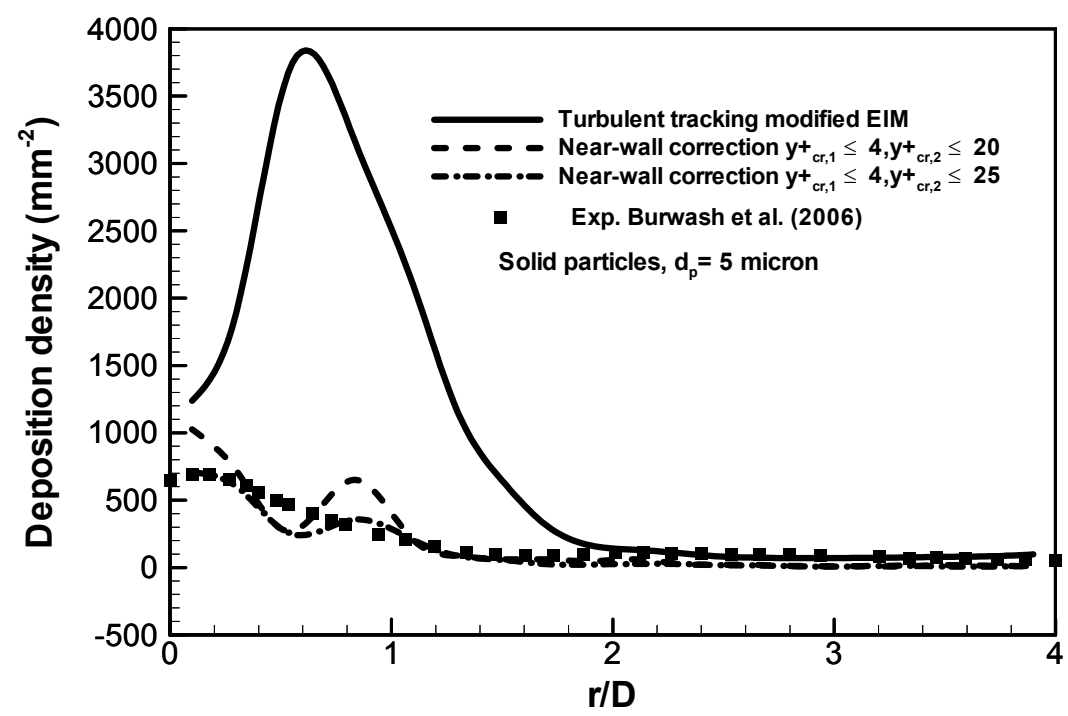

Figure 5.11. Deposition density for $L / D=6$ from turbulent tracking and near-wall correction with the new function at different $y+$ using RSM-BSL/modified EIM. 
In general, the differences that appear between the present simulation results and the experiment for all $L / D$ cases might be due to the combination of the effects presented in Subsection 4.9.2.2.

\subsection{Sensitivity of the Lagrangian time scale constant}

The effect of the Lagrangian time scale constant $C$, which is used in the Lagrangian time scale equation (Equation 3.17), is investigated using the turbulent tracking and the nearwall correction for three different values of $C=0.1,0.2$ and 0.3. Figure 5.12 shows the deposition density obtained by turbulent tracking and near-wall correction at $\mathrm{y}+\leq 15$ for $L / D=2$. As can be seen in the figure, when the constant $C$ is increased, the deposition density levels using turbulent tracking (bold lines) are generally increased. In other words, higher values of $C$ lead to a larger turbulent dispersion, which in fact causes more deposition for the turbulent tracking.

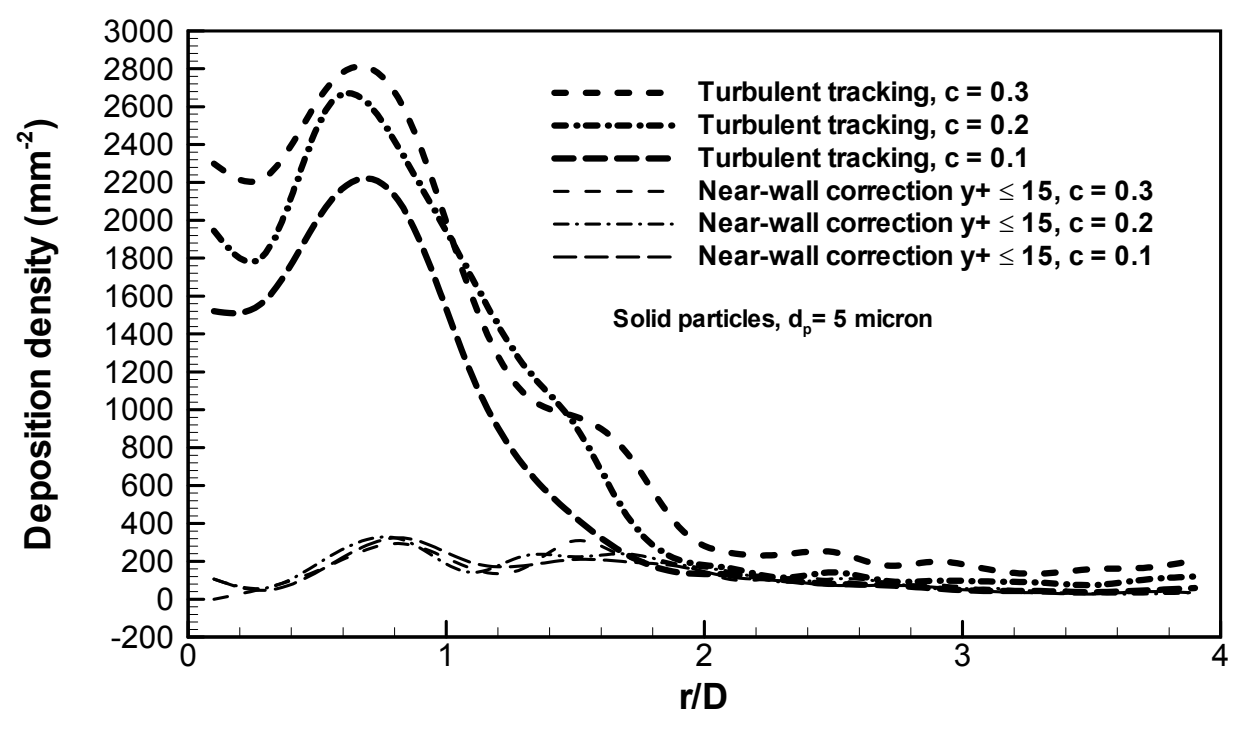

Figure 5.12. Sensitivity of the Lagrangian time scale constant. 
It can be noted that $C=0.1$ leads to the lowest deposition for turbulent tracking. However, the near-wall correction (thin lines) is insensitive to the different values of $C$ as shown in the figure. Therefore, since $C=0.1$ yields lower deposition levels for turbulent tracking, the value of $C=0.1$ is adopted in the present study to compute the Lagrangian time scale when RANS RSM/ modified EIM was used to predict the particle deposition.

\subsection{Effect of isotropic eddy lifetime}

The deposition density, which was obtained by turbulent tracking and near-wall correction at $\mathrm{y}^{+} \leq 15$, was tested by using the isotropic eddy lifetime scale $\tau_{e}=0.2 k / \varepsilon$ (Equation 3.15), where the kinetic energy, $k$, is calculated as $k=\left(\overline{u^{\prime 2}}+\overline{v^{\prime 2}}+\overline{w^{\prime 2}}\right)^{0.5}$. The

results are presented and compared to the case with $\tau_{e}=0.2 \overline{w^{2}} / \varepsilon$ (anisotropic eddy lifetime) in Figure 5.13 for the $L / D=2$ case. As can be seen in the figure, the deposition densities that are obtained by turbulent tracking using isotropic and anisotropic eddy lifetime scales are quite similar.

The deposition densities obtained by near-wall correction at $\mathrm{y}^{+} \leq 15$ using the isotropic and anisotropic eddy lifetime scales show an almost insignificant difference as shown in the figure. These results indicate that the isotropic eddy lifetime does not make significant difference and that the anisotropic eddy lifetime is quite adequate. This is consistent with the previous simulation of Burry and Bergeles (1993), who concluded similar results for particle dispersion in free jet flow. 


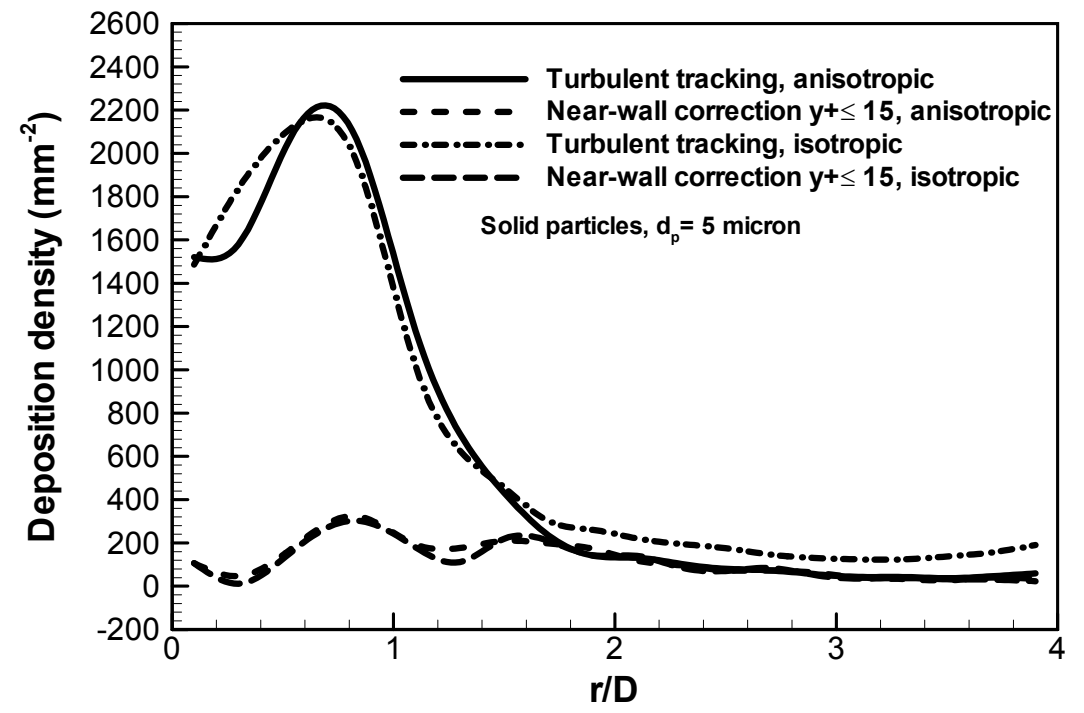

Figure 5.13. Effect of isotropic and anisotropic eddy lifetime scales on deposition.

\subsection{Particle distribution over the impingement surface}

Figure 5.14 shows the particle deposition patterns using turbulent tracking and near-wall correction (Equations 5.2-5.5) for $L / D=2,4$ and 6. It can be noted in Figures 5.14a-5.14c that the particles are intensely deposited in and around the stagnation point over the impingement wall for all $L / D$ cases. This behavior, especially for $L / D=2$, is different from the experimental pattern of Burwash et al. (2006) shown in Figure 4.20. This behavior results because of the high turbulence levels of the RMS normal-to-wall fluctuating component that is generated by the RSM-BSL model in region near the impingement wall and close to the stagnation point. When the near-wall correction is used, the deposition is generally reduced. For $L / D=2$, Figure $5.14 \mathrm{~d}$, the deposition is improved where most of the particles deposit around the stagnation point forming a ring pattern, which is qualitatively resembles the experimental pattern shown in Figure 4.20. It 
can be noted that the ring deposition pattern has a dense annular deposition area that extends outward from approximately $x / D$ or $y / D \approx \pm 0.5$ to \pm 2 .

Similarly for $L / D=4$, Figure 5.14e, the deposition is improved where most of the particles deposit making a ring-like pattern with a dense annular area around the stagnation point. However, this annular area is shrinking such that it extends to a smaller range than in $L / D=2$, i.e., it extends from approximately $x / D$ or $y / D \approx \pm 0.5$ to \pm 1.5 . This dense annular area occurs inside the deposition peak that is shown for the deposition density curve at $\mathrm{y}^{+} \leq 12$, which is shown in Figure 5.10.

For larger nozzle-to-surface distance, $L / D=6$, the deposition is reduced significantly and thus improved over the prediction that is obtained by turbulent tracking. As can be seen in Figure 5.14f, most of the particles deposit in and around the stagnation point, which is a region of maximum deposition density as shown in the deposition density curve in Figure 5.11. By comparing the patterns in Figures 5.14d-5.14f, it is clearly noted that the area of deposition around the stagnation point is moving towards the stagnation point as the nozzle-to-surface distance is increased. This behavior is because of the nozzle-to-surface distance effect, where high turbulence levels are produced near the impingement wall and close to the stagnation point as the $L / D$ distance is increased. Therefore, more deposition results in and around the stagnation point for $L / D=6$ compared to $L / D=4$ and 2. It can be noted, from these predictions for all $L / D$ cases, that the particle deposition patterns are axisymmetric as expected. 

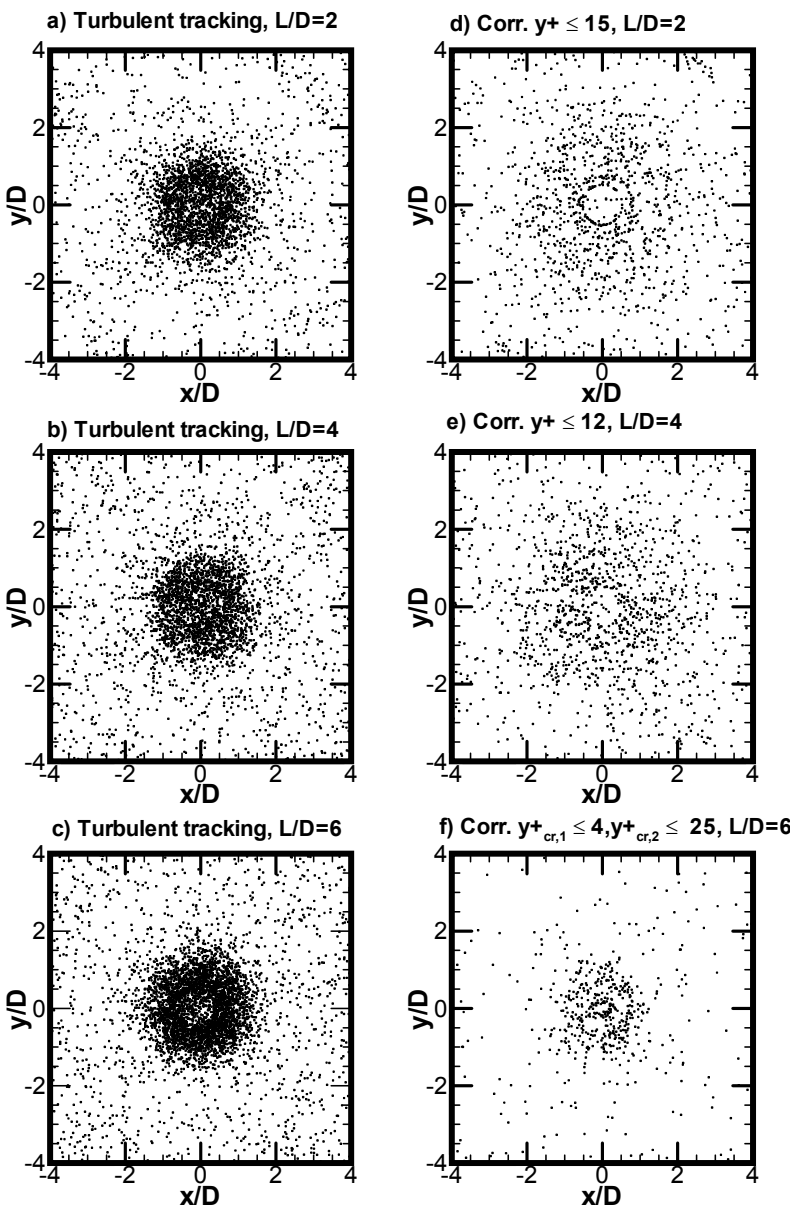

Figure 5.14. Particle deposition patterns for $L / D=2,4$ and 6 plotted for: $(a, b, c)$ turbulent tracking, and (d,e,f) near-wall correction.

\subsection{Comparison of SST/EIM and RSM-BSL/EIM}

Although both RANS models (SST and RSM-BSL) and modified EIM in conjunction with near-wall correction yield significant improvements for the particle deposition, there are some differences between RANS SST and RANS RSM-BSL models. Figure 5.15 shows a comparison of particle deposition density using RANS SST/EIM and RANS RSM-BSL/EIM, as obtained by turbulent tracking and near-wall correction, for $L / D=2$. As can be noted in the figure, the deposition density obtained with the turbulent tracking using SST/EIM and, to a lesser extent, RSM-BSL/EIM has a close prediction when 
compared to the experimental data of Burwash et al. (2006) at approximately $r / D>1.8$. Indeed, both models, SST/EIM and RSM-BSL/EIM, overpredict the experiment at $r / D<$ 1.8; however, RSM-BSL/EIM yields a higher deposition density than the SST/EIM, which is probably due to the absence of stress limiters in the RSM-BSL model. When the near-wall correction function is used, the deposition density prediction is highly improved using both models, although the peak at about $0.5 \lessgtr r / D \lesssim 1.6$, which is reduced in RANS RSM-BSL/EIM more than in RANS SST/EIM.

Similarly for $L / D=4$ in Figure 5.16, whereas the SST/EIM predicts a higher deposition density over the entire range of $r / D$ using the turbulent tracking, the RSMBSL/EIM predicts the deposition density quite well at $r / D>1.8$. However, it shows a higher prediction than the experiment and the SST model at $r / D<1.8$, similar to $L / D=2$, due to the absence of the stress limiter. When the near-wall correction function is used, the predictions from both models are significantly improved. It can be noted that the prediction using RSM-BSL captures the experiment much better than the prediction using the SST model.

In the same manner for $L / D=6$, it can be noted from Figure 5.17 that the RSMBSL/EIM predicts a higher deposition density than the SST/EIM using the turbulent tracking at $r / D<1.8$. However, its prediction agrees fairly well with the experiment at $r / D>1.8$ unlike SST/EIM, which predicts higher deposition due to excessive production of kinetic energy despite the stress limiters in the model. As the near-wall correction function is used, the predictions from both models are significantly improved. It can be 


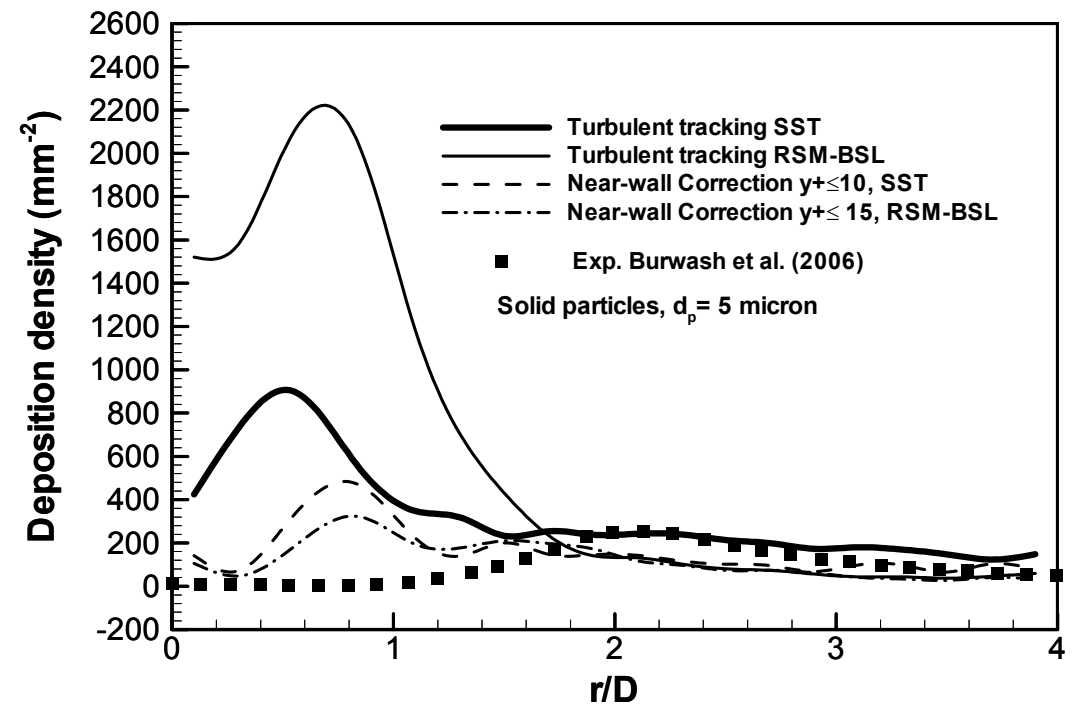

Figure 5.15. Comparison of deposition density for $\mathrm{L} / \mathrm{D}=2$ using SST and RSM-BSL along with modified EIM.

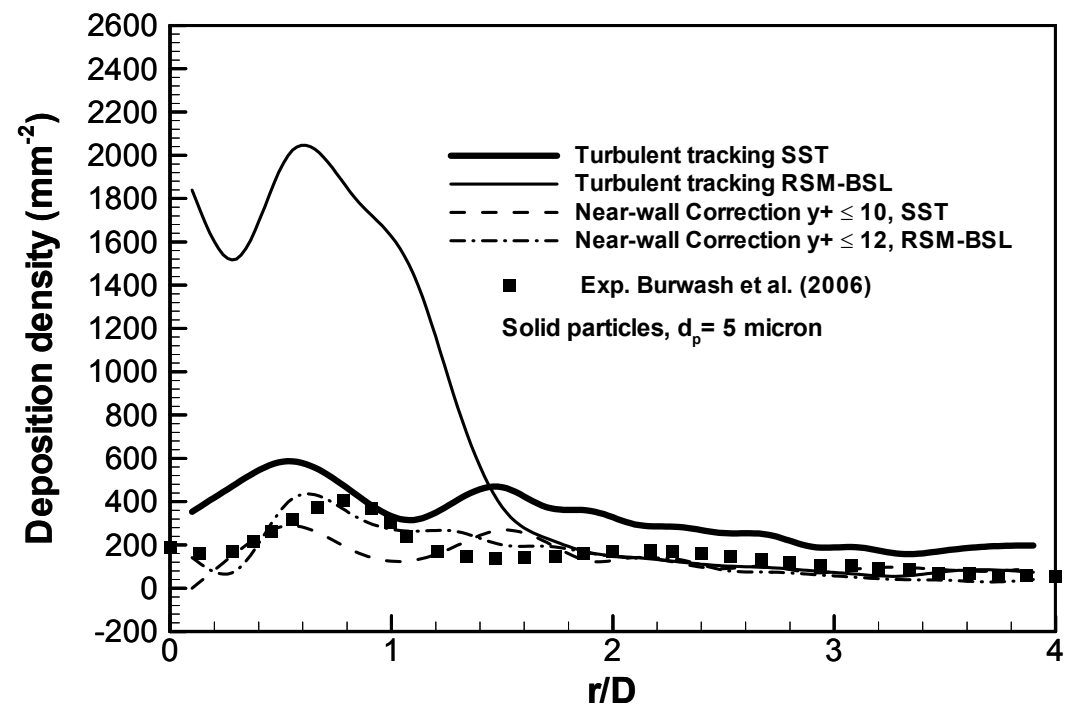

Figure 5.16. Comparison of deposition density for $\mathrm{L} / \mathrm{D}=4$ using SST and RSM-BSL along with modified EIM. 


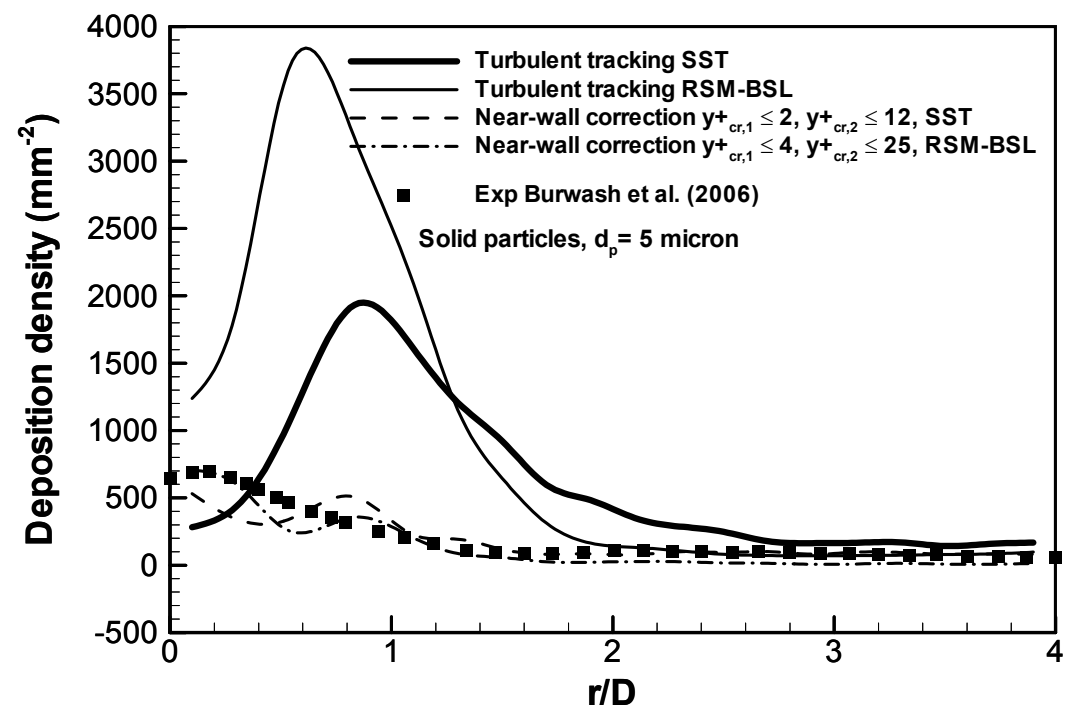

Figure 5.17. Comparison of deposition density for L/D $=6$ using SST and RSM-BSL along with modified EIM.

noted that the SST/EIM (dash lines) yields a good prediction in the wall jet region, but still overpredicts the experiment slightly before it shows a slight underprediction close to the stagnation point (at approximately $r / D<0.5$ ). The prediction using RSM-BSL/EIM (dot dash lines) captures the experiment accurately, even the peak close to the stagnation point, unlike SST/EIM, although the small underprediction that is noted at approximately $0.4 \lesssim r / D \lesssim 0.7$

From Figures 5.15, 5.16 and 5.17, the results suggest that both models, using the turbulent tracking, yield a higher deposition than the experiment for all $L / D$ cases at the beginning of the wall jet region and as the stagnation point is approached. This shows the importance of using the near-wall correction functions in order to predict adequate particle deposition levels. Also, these results suggest that the RSM-BSL/EIM shows significant improvement over the SST/EIM when the near-wall correction is used. The 
reason for this improvement, in addition to the good performance of the near-wall correction damping functions that take the near-wall effects into account, is the RSMBSL performance that provides a more accurate description of the turbulent flow field near the impingement wall. The next paragraphs describe the performance of the two models.

The differences in the deposition results that are obtained using SST/EIM and RSM-BSL/EIM are more appreciable by examining the performance of the SST and RSM-BSL models. The contours of the velocity magnitude and the turbulent kinetic energy in region close to the wall $(z / D<2$, and a dimensionless distance from the jet centerline axis, $x / D<2.5$ ) using the RANS SST and RANS RSM-BSL models for $L / D=$ 2, 4 and 6 are shown in Figures 5.18 and 5.19, respectively. As can be seen in the figures, the contours of velocity magnitude from both models demonstrate a similar development of the flow as it reaches the impingement wall and then turns sideways over the impingement wall (wall jet region). However, the SST model differs from the RSM-BSL model in the width of the free shear layer as the flow approaches the wall and also in the extent of the recirculation zones in the wall jet region. It can be noted that in the SST model, the free jet shear layer width has a larger expansion than that in the RSM-BSL model as shown in the velocity contours (Figure 5.18). This implicitly affects the flow development in the subsequent stages. Also, the SST model predicts slightly larger recirculation zones than the RSM-BSL model very close to the impingement wall as shown in the kinetic energy contours (Figure 5.19).

This makes the SST model prediction is not representing the flow behavior adequately due to the insensitivity of the streamline curvature and the shortcoming in 
accounting for the anisotropy effects. This in turn affects the deposition prediction, even with the use of the near-wall correction, and in spite of the stress limiters used in the SST model. However, although the RSM-BSL model yields higher fluctuating velocities (high kinetic energy) close to the wall, as noted in the kinetic energy contours, it still preserves certain features such as accounting for anisotropy, turbulent transport and streamline curvature. These features along with the use of the near-wall corrections, which are implemented in the tracking code (modified EIM), lead to significant improvement in the predictions of the particle deposition. 

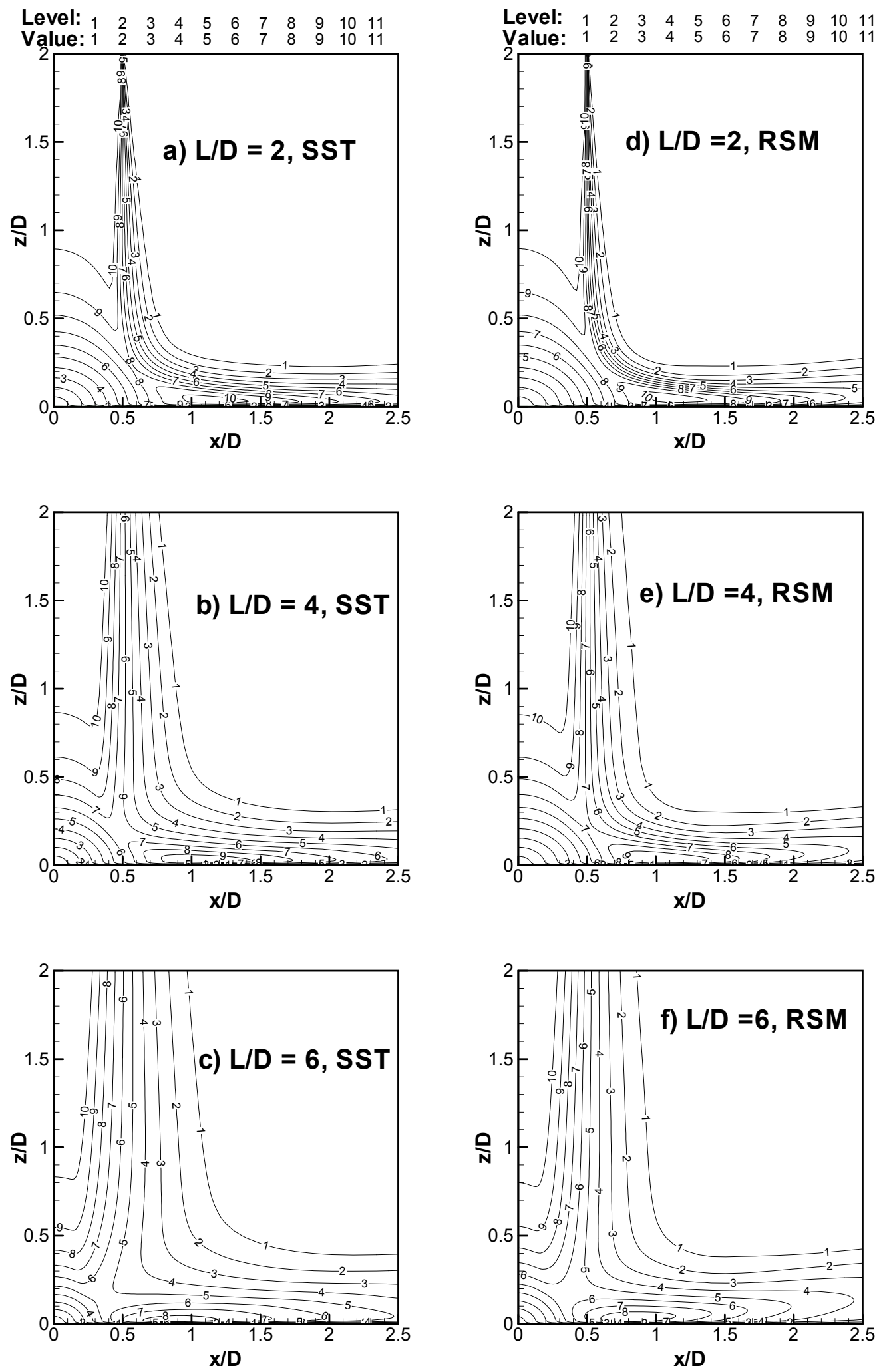

Figure 5.18. Velocity magnitude contours ( $x-z$ plane, $y=0)$ for $L / D=2,4$ and 6 using SST and RSM-BSL model. Value is in $\mathrm{m} / \mathrm{s}$. 

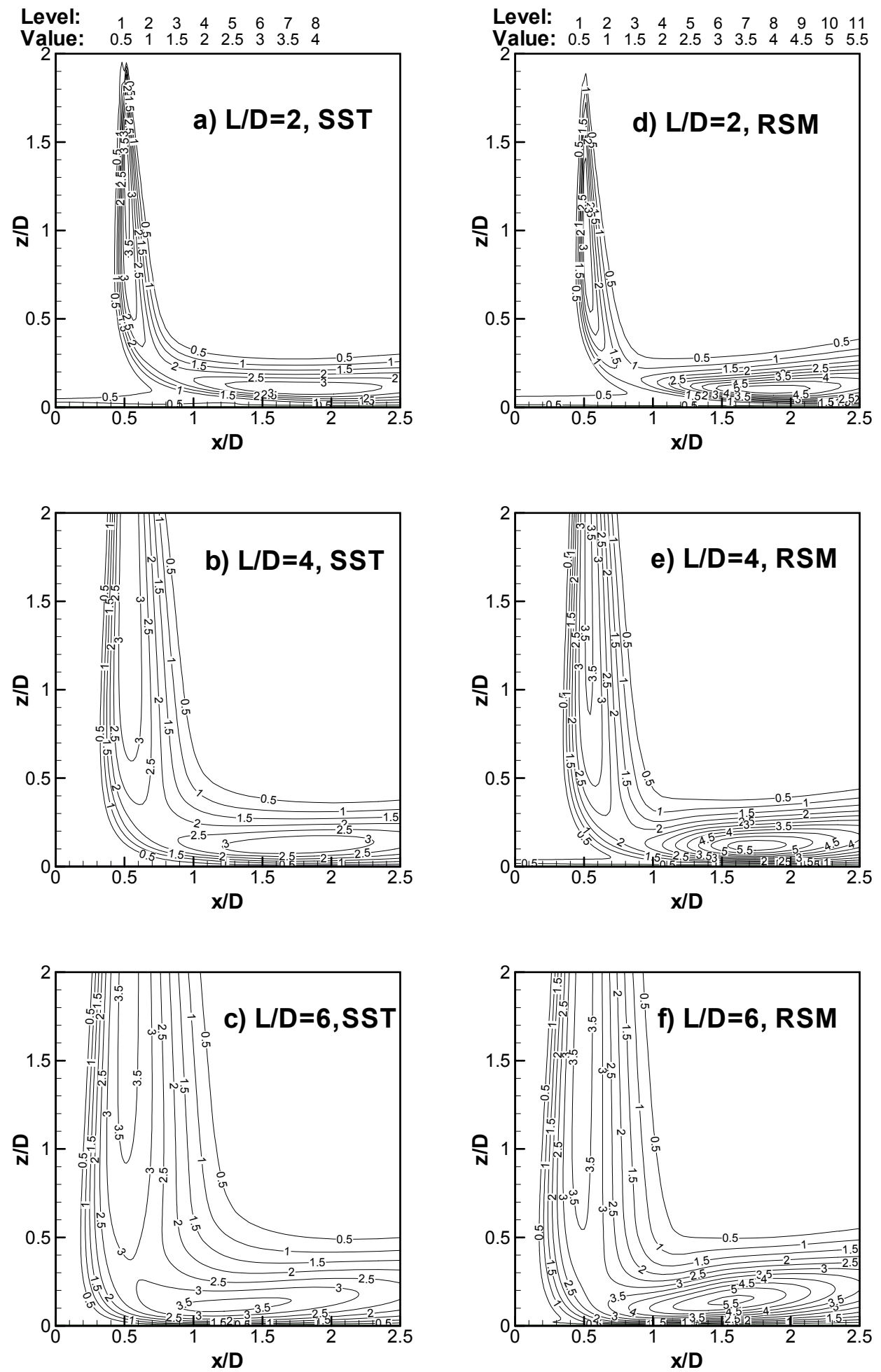

Figure 5.19. Kinetic energy contours $(x-z$ plane, $y=0)$ for $L / D=2,4$ and 6 using SST and RSM-BSL model. Value is in $\mathrm{m}^{2} / \mathrm{s}^{2}$. 


\subsection{Conclusion}

In this chapter, another approach, RANS RSM-BSL/modified EIM based on solving individual equations for Reynolds stresses, was used to predict the particle deposition. However, despite accounting for the anisotropy of turbulence, the deposition obtained by turbulent tracking "without correction" has significant overprediction as the stagnation point is approached for all the $L / D$ cases. By implementing the near-wall correction function based on the impinging jet flow characteristics, which were obtained from RANS RSM-BSL model, using similar procedure as the one adopted when RANS SST was used, significant improvement was obtained for all the $L / D$ cases. Also, the particle deposition patterns using the near-wall correction were consistent with the deposition density curves for all the $L / D$ cases.

The predicted deposition density, in general, showed close agreement with the experimental data for all the $L / D$ cases more than the deposition density predicted using RANS SST/EIM. The reason for this is because RANS RSM-BSL model preserves certain features including anisotropy, turbulent transport, and streamline curvature, which are along with the use of the near-wall correction, lead to significant improvement over RANS SST/EIM. 


\section{Chapter 6: Results Using LES}

Having explored the particle deposition in the impinging jet flow with two different approaches in framework of RANS, RANS SST and RANS RSM models in conjunction with EIM along with the "near-wall correction" technique, the next objective is to investigate the particle deposition using a more advanced approach. The accurate prediction of the complex flow fields, such as the impinging jet flow, encountered in different industrial systems is essential to gain a better understanding of the phenomena governing particle transport and deposition as well as to ultimately improve the deposition prediction. Such a prediction requires high fidelity computational fluid dynamics (CFD) analysis tool that can accurately account for the particle-turbulence interactions. Solving the unsteady Navier-Stokes equations, as in LES, can improve the modeling of the two-phase flow and enhance our understanding of the complex particleturbulence interactions.

In this chapter, the particle deposition in impinging jet flow with nozzle-to-surface distance of $2, L / D=2$, using perturbed inflow boundary conditions, is investigated by LES. Two different procedures for applying the perturbations at the inflow boundary were tested and their effects on the particle deposition are evaluated and compared. Also, particle deposition patterns are presented. The present particle simulation results were validated by comparing with the experiment of Burwash et al. (2006). In addition, the particle LES simulation results were compared with the particle simulation results using RANS RSM-BSL/EIM. 


\subsection{Computational domain and numerical parameters}

In the present study, the computational dimensions were made based on the experiment of Burwash et al. (2006). Figure 6.1 shows a schematic of the computational domain and the dimensions used. In this case study, the height $L$ is kept constant at $30 \mathrm{~mm}$, where the nozzle-to-surface distance is equal to $2(L / D=2)$. In addition, the inlet boundary was moved upstream the nozzle exit in order to have better representation of the inflow condition. Including the nozzle in the computational domain was to allow the large structure vortices to be initiated, by using imposed perturbations, at the jet exit and thus evolve downstream as the jet is developed.

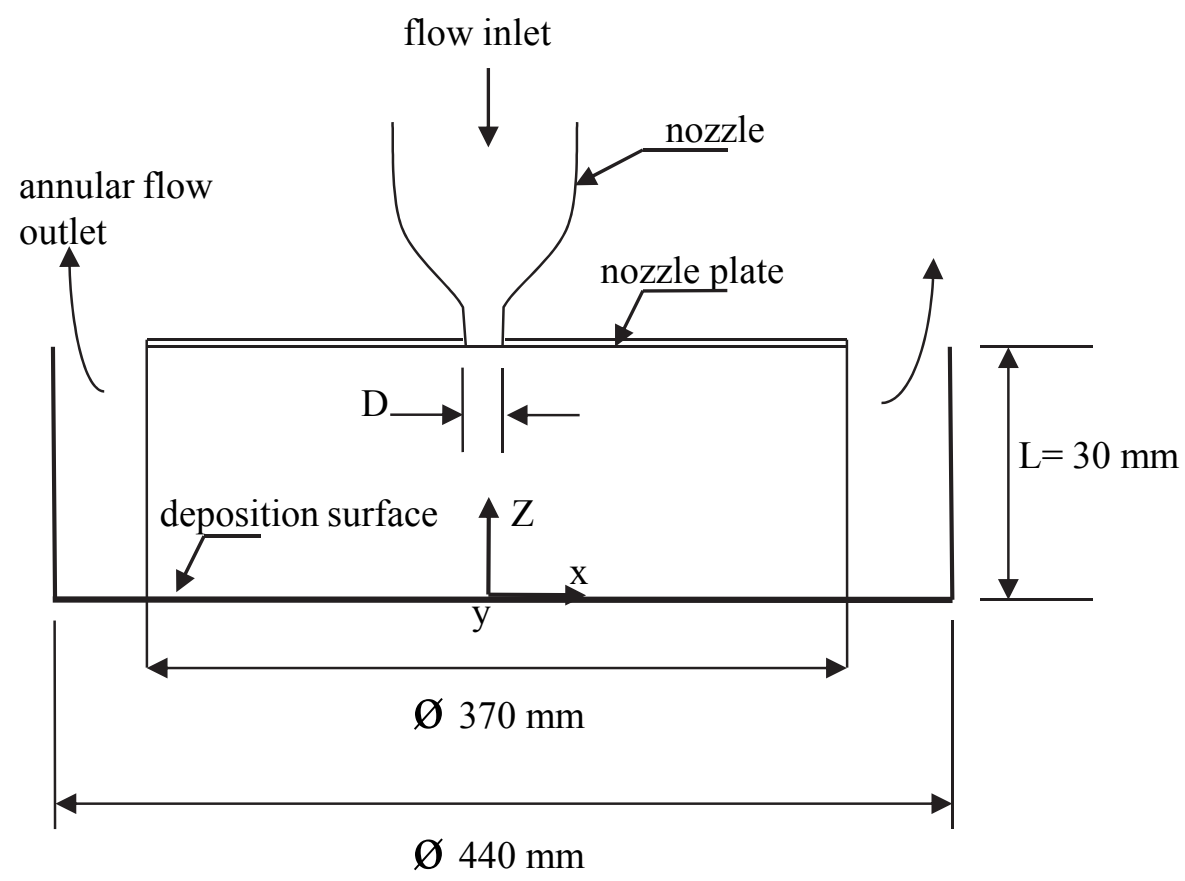

Figure 6.1. Schematic of the computational domain of the impinging jet based on the experiment of Burwash et al. (2006).

As stated before, LES computations are more expensive than RANS computations. Since the LES method aims at resolving the scales of motion, it requires extremely fine 
grid resolution, including the near-wall region, approaching that of DNS. The computational domain was constructed using CFD-ICEM (Ansys Inc.) mesh generator code. The computational domain was composed of structured multi-block mesh. In this mesh, 22 blocks were used. Grid points were accumulated at the walls of the nozzle, and at the bottom, top, and side walls of the computational domain (Kubacki and Dick, 2010a). Moreover, the grid points were accumulated in the shear layer of the jet since these are regions of high velocity gradients. Also, the grid points were clustered in the central region of the geometry (from the inlet up to the stagnation point) and towards the impingement wall (near-wall region) with a grid expansion factor of 1.01. Figure 6.2a shows a top view of the computational domain, where the grid points were accumulated in the central region of the domain. Figure $6.2 \mathrm{~b}$ shows a cluster of grid points near the impingement wall. Discontinuities in node spacing at the boundaries were avoided in order to have smooth connections. In order to ensure numerical accuracy, the sensitivity of the results to the grid sizes was investigated as shown in Section 6.2.

The inflow conditions for unsteady simulations, such as LES, are of primal importance because the evolution of the jet is strongly affected by the inflow conditions. Therefore, random perturbation (random fluctuations) with intensity of $10 \%$ of the nozzle velocity, added to the nozzle velocity, was applied to the inflow condition in the normal direction (normal-to-wall) at every time step upstream of the nozzle exit. In addition, random perturbations with intensity of $10 \%$ of the nozzle velocity were applied in the transverse and spanwise directions at every time step. The randomly perturbed inflow boundary condition is defined as 


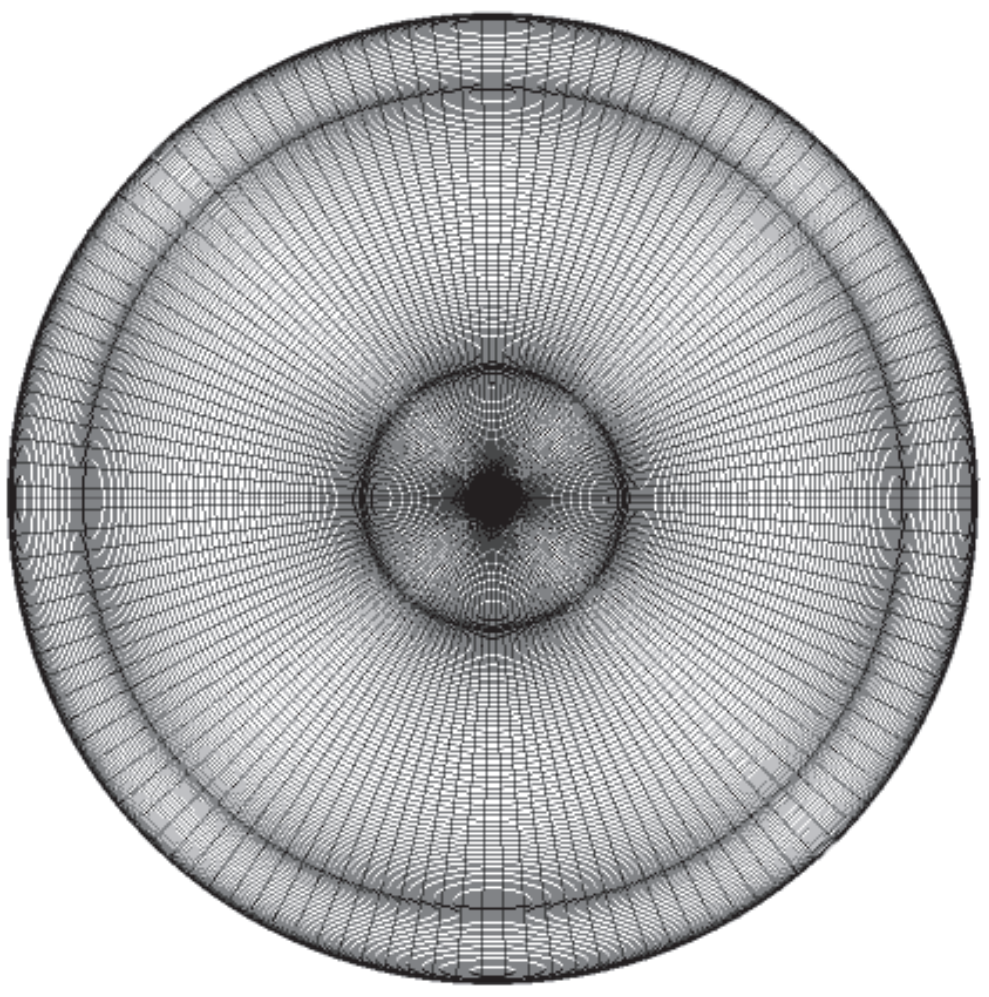

a) Top view of the computational domain.

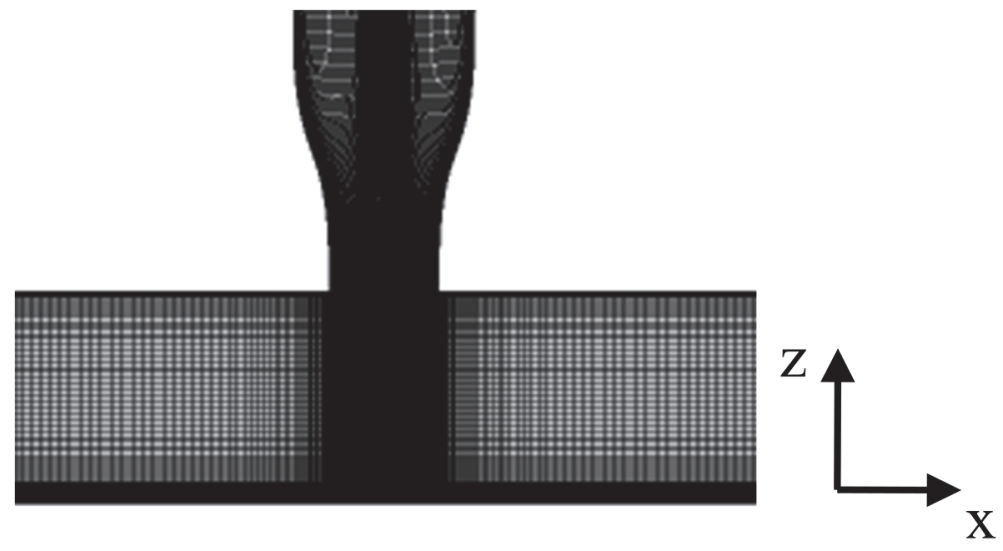

b) Zoom of the normal-to-wall mesh in $x-z$ plane.

Figure 6.2. Grid of the computational domain of the impinging jet used for LES calculations for: (a) Top view of the computational domain, and (b) Zoom of the normal-to-wall mesh in $x-z$ plane. 
$U(x, y, t)=A U_{n o z z}(\operatorname{gasdev}(x, y, t))$

$V(x, y, t)=A U_{n o z z}(\operatorname{gasdev}(x, y, t))$

$W(x, y, t)=U_{\text {nozz }}+A U_{\text {nozz }}(\operatorname{gasdev}(x, y, t))$

where $U_{\text {nozz }}=3.63 \mathrm{~m} / \mathrm{s}$ is the nozzle velocity in the normal-to-wall direction. $A$ is the turbulence intensity equal to $10 \%$ of the nozzle velocity, and gasdev is the Gaussian function that provides random numbers with zero mean and unit standard deviation. The perturbed inflow boundary condition was generated using an in-house code. At every time step, the perturbed inflow was generated at the inlet plane and then saved in a file. Then, these files were fed as inflow while the simulation was running. Each file contains perturbed inflow information for 1000 time steps. Therefore, this requires running the simulation in segments where each segment is 1000 time steps. The reason for this procedure is because of the CFX code requirement that allows the use of specific number of files during the simulation for a certain range of time steps. This procedure is denoted by dynamic perturbation.

For flexibility reasons, another procedure was adopted in the present study in order to generate inflow boundary condition by using random fluctuations. In this procedure, the random fluctuations were generated once and then applied in every time step throughout the simulation. This procedure is denoted by "constant perturbation". The rest of the boundary conditions include the standard no slip condition at the walls, and zero gage pressure opening outlet was applied at the outlet boundary.

In the present study, the governing equations of the fluid phase were solved numerically using CFX 12.0 (Ansys Inc.). Incompressible airflow at $25 \mathrm{C}^{\mathrm{o}}$ was assumed 
in the solution. For all LES computations in the present study, a time step of $\Delta t=9 \mu \mathrm{s}$ was chosen. The time step was determined with respect to the explicit time-marching scheme and temporal resolution requirement of LES Courant-Friedrich-Levy (CFL) number that requires $\mathrm{CFL} \leq 1$. In the present computations value of CFL number less than 0.8 was maintained through the simulations. A central differencing scheme was used in the LES computations. For the time advancement, a second order accurate implementation using a backward implicit difference formula was used. The present simulations were initialized from converged RANS computations. In order to eliminate the effect of initial conditions, 40,000 time steps were used in the present computations. The computations were performed on a cluster having 20 nodes.

Similar to RANS/EIM particle simulations, 10,000 particles of $5 \mu \mathrm{m}$ were used in the LES particle simulations. The particles were released and fully tracked at one time step in a frozen domain, and then deposition statistics were recorded. The particles were randomly and uniformly distributed in space at the inlet. Initial particle velocities were set equal to the fluid velocity.

\subsection{Effect of grid size on deposition}

In the present analysis, three different grid sizes of $820,185,2,429,407$ and $3,278,328$ nodes were tested and compared. The third grid was refined with this large number of nodes such that it allows for a sufficient comparison to be made regarding the accuracy of the results, thus providing a greater resolution of the results. In this sense, the effect of the computational grid size on the particle deposition is conducted and shown in Figure 6.3 . 


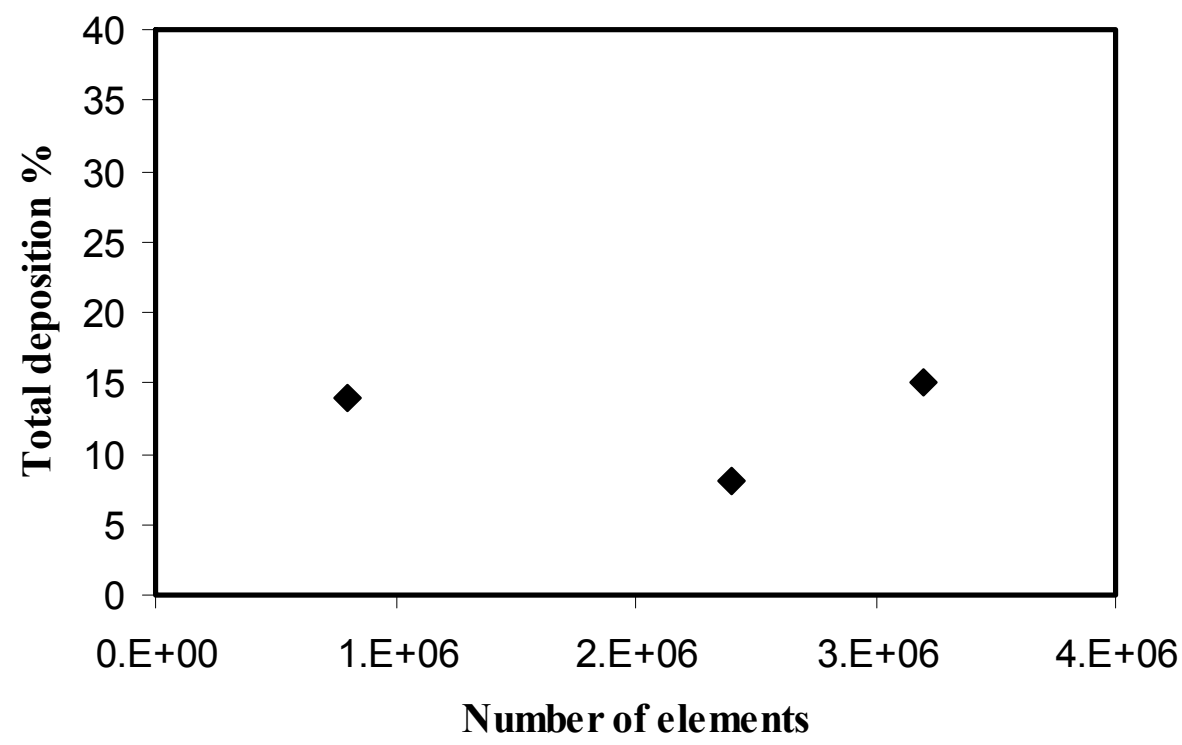

Figure 6.3. Effect of grid size on the particle deposition using LES.

As can be seen from the figure, when the number of nodes was increased to $2,429,407$ nodes, the particle deposition is reduced slightly. However, as the number of nodes is further increased to $3,278,328$ nodes, the deposition is increased showing very close value to the deposition when 820,185 nodes were used. This indicates that the grid convergence is obtained despite the very slight increase, which is expected as the number of nodes was greatly increased. The difference (between the first and the third grids) in percentage total deposition is approximately within $1 \%$, indicating that the grid resolution for the refined mesh is adequate. Therefore, based on the above discussion, the refined grid size is chosen for the present analysis. The reason for this choice is associated to the LES requirements, where the refined mesh performs better in terms of the results accuracy. 


\subsection{Deposition results}

Figure 6.4 shows the deposition density curves obtained by LES using constant and dynamic perturbations in comparison with the deposition density curves from RANS RSM-BSL/EIM using turbulent tracking (bold solid line) and near-wall correction (thin solid line). Part of the turbulent tracking curve (as the stagnation point is approached) is not shown in the figure because its range is outside the bound used. As can be seen from the figure, the deposition density results from LES using constant (dash lines) and dynamic (dot dash lines) perturbations are better predicted and qualitatively have the closest prediction to the experiment of Burwash et al. (2006) (dark square symbols) over most of the $r / D$ range.

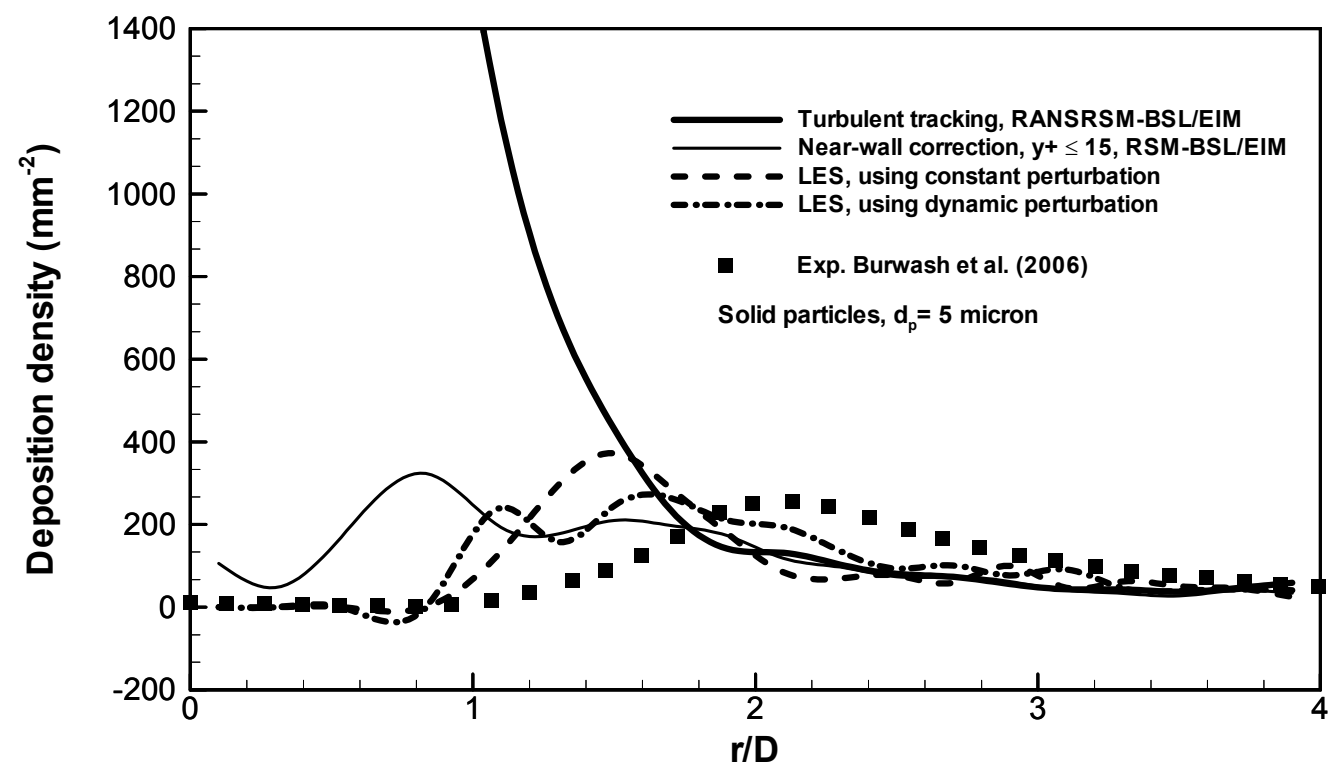

Figure 6.4. Particle deposition density using LES compared with RANS calculations.

It can be noted that the behaviors of the LES results resembles the experiment qualitatively in most of the regions over the impingement wall, i.e., very close to the stagnation point and along the wall jet region, although the deposition peak (at 
approximately $r=1.6 D$ ) occurs slightly upstream of the experimental peak (at approximately $r=2.1 D$ ) for both constant and dynamic perturbations. The reason of this is probably because of the large structure vortices. As the flow is deflected upon the presence of the wall, the large structure vortices, which form as the wall jet develops and the kinetic energy increases due to shear flow, cause more particles to deposit in this region just upstream the experimental peak. Moreover, as these structures develop downstream the wall jet region, they initiate secondary vortices that lead to more deposition before they weaken in the far downstream and the deposition is reduced below the experiment at about $2.1 \leq r / D \leq 2.8$. It can be noted that the LES with constant perturbation, unlike the LES with dynamic perturbation, yields higher deposition at approximately $1 \leq r / D \leq 1.8$. This appears to be due to the development of more large structures in this region and subsequently secondary vortices that are weakened quickly. As a result, this produces less deposition than the experiment and the LES with dynamic perturbation as the flow develops farther downstream the wall jet. Generally, it is conjectured that the difference between the present LES and the experiment can be attributed to the variations between the actual inflow conditions in the experiment and those used for the simulation.

It can be noted that, overall, the LES with dynamic perturbation has better performance than that with constant perturbation. This is because the dynamic perturbation demonstrates physically relevant turbulence features since the random perturbation is generated at every time step unlike the constant perturbation. In the constant perturbation, the random perturbation, which is generated at the first time step, is applied in the subsequent time steps of the simulation. It should be mentioned here that 
the two ways were used in the present research in order to develop the turbulence structures that represent the energetic scales of the flow and generate three dimensional effects. However, the constant perturbation was adopted because it is less expensive (requiring approximately $384 \mathrm{CPU}$ hours) than the dynamic perturbation.

In order to apply the dynamic perturbation as an inflow boundary condition, it is required to run the simulation of 40,000 time steps in segments due to the CFX requirement. Every segment is 1000 time steps and uses one file that stores the time series of velocity fluctuations for every time step. Therefore, this requires approximately 85 days to run the LES simulation for 40,000 time steps. This indicates that LES is considerably much more expensive when compared to the RANS/EIM particle simulations.

From Figure 6.4, it can be noted that the LES predicts the particle deposition density, by far, better than the turbulent tracking, which yields an unrealistic behavior near the stagnation point and at the beginning of the wall jet region. Also, although using the near-wall correction relatively improves the deposition prediction, it still gives a higher deposition than the experiment and LES close to the stagnation point and at the beginning of the wall jet. This is probably attributed, in addition to the limitation of the EIM, to the shortcoming of RANS (with its model RSM-BSL). RANS provides only limited information about the turbulence characteristics and almost no details on the unsteady large-scale structures of the flow field unlike LES, which is designed to simulate the unsteady behavior of those structures of the flow field and preserve the characteristics of the vortex. This directly affects the deposition prediction, which depends mainly on the correct flow field representation. 


\subsection{Particle distribution over the impingement surface}

Figures $6.5 \mathrm{a}-6.5 \mathrm{~d}$ show the particle deposition patterns for $L / D=2$ resulting from RSMBSL/EIM, using turbulent tracking and near-wall correction (NWC), and LES with dynamic and constant perturbations. As can be seen from these figures, the differences between RSM-BSL/EIM and LES are evident. It can be noted that the deposition patterns from LES, unlike the turbulent tracking and near-wall correction, have the closest pattern to the experiment shown in Figure 4.20. The particles deposit around the stagnation point in a ring pattern, similar to RSM-BSL/EIM with near-wall correction, but the annular deposition area from LES is not as dense as in the near-wall correction. Although the annular deposition area from LES with dynamic perturbation (Figure 6.5c) extends outward from approximately $x / D$ or $y / D \approx \pm 1$ to \pm 2.6 , the corresponding deposition area from LES with constant perturbation (Figure 6.5d) is less extended outward. However, the predictions of the particle deposition patterns are axisymmetric similar to the experiment as expected.

Moreover, from the deposition patterns that are produced by LES, it can be noted that some particles, as they deposit around the stagnation point, mass in some spots because they are influenced by the presence of the secondary and large-scale structure vortices, i.e., the particles follow these structures (Fan et al., 2001). This feature is not demonstrated by RSM-BSL/EIM especially when the near-wall correction is used. This indicates that the particles, due to their small inertia, follow closely the flow field before they deposit on the wall. 


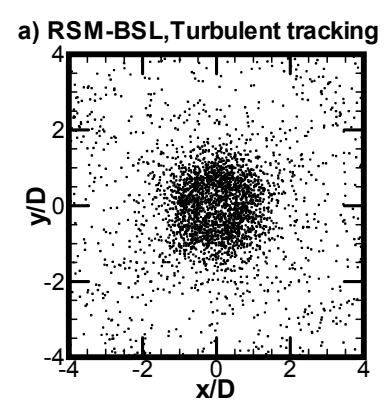

b) RSM-BSL, NWC

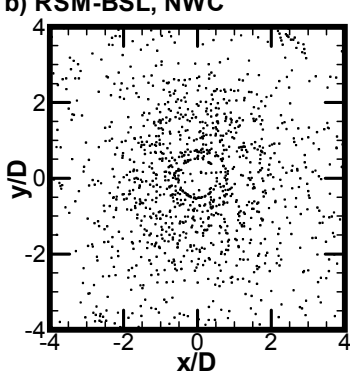

c) LES, dynamic perturbation

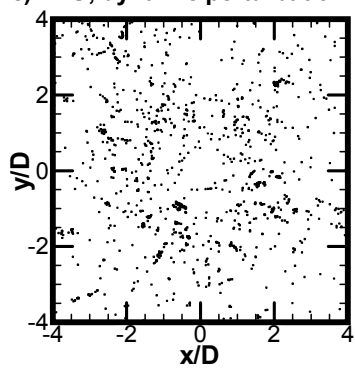

d) LES, constant perturbation

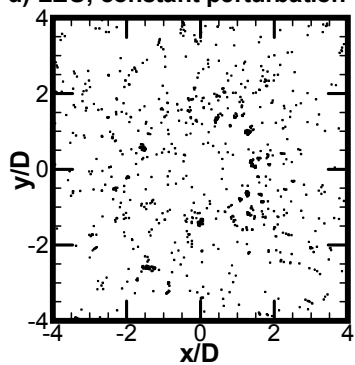

Figure 6.5. Particle deposition patterns for $L / D=2$ plotted for: (a) turbulent tracking using RANS/EIM, (b) near-wall correction using RANS/EIM, (c) using LES with dynamic perturbations, and (d) LES with constant perturbations. 


\subsection{Conclusion}

In this chapter, the particle deposition was investigated for the nozzle-to-surface distance of 2 using LES. In order to have better representation of the inflow boundary condition and to allow the large structure vortices to be initiated at the jet exit, random perturbations were applied upstream the nozzle exit (jet exit). Two different procedures including dynamic and constant perturbations were applied. In dynamic perturbation, the random fluctuations were generated at every time step throughout the simulation, however; in constant perturbation, the random fluctuations were generated once and then the same fluctuations were used in the subsequent time steps.

The LES deposition results including the deposition density and the deposition patterns using dynamic and constant perturbations were in close agreement with the experimental data despite the small difference. The LES with dynamic perturbation has better performance than that with the constant perturbation since the dynamic perturbation exhibits physically relevant turbulence characteristics where the random perturbation is generated at every time step. Indeed, the LES predicts the particle deposition much better than the turbulent tracking and has significant improvement over RANS RSM-BSL/modified EIM with the near-wall correction. This is because the LES simulates the unsteady behavior of the large-scale structures and preserves the characteristics of the vortex. 


\section{Chapter 7: Conclusions and Future Work}

\subsection{Conclusions}

A comprehensive prediction of aerosol particle dispersion and deposition in impinging jet flow has been conducted. In addition to the classical EIM, which is implemented in CFX code, a new in-house tracking code for particle-laden impinging jet flow using modified EIM was developed to simulate the particulate phase in three cases of nozzle-to-surface distances of $L / D=2,4$ and 6 . Particle deposition density results using turbulent tracking from RANS SST model, along with the classical and modified EIM, generally showed higher predictions than experimental data for all $L / D$ cases, although the predictions using modified EIM demonstrated lower levels of the deposition density profiles. These higher predictions were found to be caused by the isotropy assumption. Using the eddy ratio correction implemented through classical EIM led to a remarkable improvement in spite of some differences that still remain due to the limitations of this correction and the classical EIM.

The present analysis indicated that the nozzle-to-surface distance has a sensible effect on the particle deposition. As the $L / D$ distance is increased, more deposition occurs close to the stagnation point. This was confirmed by the deposition pattern, which exhibits a ring-like deposition with an annular area moving towards the stagnation point as $L / D$ was increased.

The present analysis revealed the fact that the appropriate modeling of the normalto-wall fluctuating velocity in the near-wall regions is crucial for accurate particle simulations. Therefore, in this regard, the near-wall correction function calibrated 
according to the impinging jet flow characteristics was adopted to correct and give an adequate representation of the normal-to-wall fluctuation. From the deposition predictions that were performed using RANS SST with and without Kato and Launder modification, along with modified EIM in conjunction with the near-wall correction, significant improvement was obtained. For the case of $L / D=2$, the near-wall-correction at $\mathrm{y}^{+} \leq 5$, when Kato and Launder modification was used, gave the closest prediction to the experiment. However, for the $L / D=4$ case without Kato and Launder modification, the near-wall correction at $\mathrm{y}^{+} \leq 10$ yielded the closest behavior to the experiment unlike the case with Kato and Launder modification.

For larger nozzle-to-surface distance, $L / D=6$, the deposition was affected by the stagnation point. Therefore, the application of the function was modified such that the function switches to use small $\mathrm{y}^{+}$values close to the stagnation point. The simulation results were close to the experiment for the case without Kato and Launder modification more than the case with Kato and Launder modification.

The present analysis using RANS RSM-BSL model, along with the modified EIM, demonstrated a higher deposition density at the beginning of the wall jet and stagnation near-wall regions in spite of accounting for the anisotropy of turbulence. However, as the near-wall correction technique was used via the calibrated near-wall function, significant improvement was obtained for all $L / D$ cases. Although a higher prediction was noted at the beginning of the wall jet due to the particle accumulation effect, deposition density results using the near-wall function showed significant improvement for the $L / D=2$ case compared to the turbulent tracking. Moreover, the predicted deposition density curves, at $\mathrm{y}^{+} \leq 12$ for $L / D=4$ and using set 2 for $L / D=6$, were in close agreement with the 
experiment more than the predictions using RANS SST/EIM in conjunction with the near-wall correction. The large eddy simulation with the perturbed inflow boundary condition for $L / D=2$ led to deposition results that are in close agreement with experimental data more than RANS RSM-BSL/modified EIM.

Generally, RANS approach with the modified EIM, along with its capability and simplicity of incorporating near-wall correction, was able to predict the deposition results close to the experiment. Also, the LES deposition results for $L / D=2$ showed that the LES is a powerful approach that permits for more accurate accounting of particleturbulence interactions. The results from this comprehensive prediction provide new insight into the behavior of the aerosol particle transport and deposition process that can enhance the understanding of deposition in impinging jet flow.

\subsection{Future work}

This research represents the first detailed study of various turbulence models (RANS SST/EIM, RSM-BSL/EIM and LES) for particle transport and deposition in turbulent jet flow impinging normally over a flat plate. Therefore, this research opens the horizon for a wide range of investigations using more advanced numerical approaches such as LES with different stochastic models and DNS that could be used for assessing the accuracy of deposition predictions in the future.

The present research has shown that EIM in framework of RANS led to significant improvement in the deposition prediction of impinging jet flow with inclusion of the near-wall correction technique. However, this approach is still limited. Although including the SGS velocities has an insignificant effect on the deposition for a small 
Reynolds number, an improved detailed study using LES with simulating the SGS effect on the particles using a stochastic model would provide more realistic results of particle deposition. The use of a coarser numerical description coupled with the stochastic modeling of the SGS can provide substantial savings in CPU time required for the computation compared to a fully resolved two-phase LES. In addition, incorporating the near-wall correction, which can account for the near-wall regions based on impinging jet flow characteristics, into the stochastic model, especially for a high Reynolds number, is important for more accurate predictions. Finally, since the prediction of the particle deposition depends heavily on an accurate fluid flow phase, a DNS study, which can account for the most accurate representation of the fluid flow with particulate phase treated in a Lagrangian context, comprises an important direction for the future research on particle deposition prediction in impinging jet flow.

The suggested future work is useful for the development of particle deposition prediction in impinging jet flow. Also, it is helpful for enhancing our fundamental understanding of the mechanisms that govern deposition in impinging jet flows. 


\section{References}

Abuzeid S., Busnaina A. A. and Ahmadi G., Wall deposition of aerosol particles in a turbulent channel flow., J. Aerosol Sci., vol. 22, no. 1, pp. 43-62, 1991.

Agnihotri V., Ghorbaniasl G., Verbanck S. and Lacor C, An eddy interaction model for particle deposition, Aerosol Sci., vol. 47, pp. 39-47, 2012.

Ahmadi G. and Chowdhury S., A rate dependent algebraic stress model for turbulence, Clarkson University, N.Y., MIE-168, 1988.

Ai W. and Fletcher T., Computational analysis of conjugate heat transfer and particulate deposition on a high pressure turbine vane, Journal of Turbomachinery, vol. 134, pp. 1$12,2012$.

Alatawi E. S. and Matida E. A. , Effect of anisotropy on particle deposition in impinging jet flow, Intr. Journal Mechanical Engineering \& Mechatronics, vol. 1, pp. 95-101, 2012.

Almeida $\mathrm{T}$ and Jaberi F., Larg-eddy simulation of a dispersed particle-laden turbulent round jet, Int. J. Heat and Mass Transfer, vol. 51, pp. 683-695, 2008.

Amano R. S., Turbulent effect on the impinging jet on flat plate, Bulletin of the JSME, vol. 26, pp. 1891-1899, 1983.

Anderson, S and Longmire, E. , Particle motion in the stagnation zone of an impinging air jet, J. Fluid Mech, vol. 229, pp. 333-366, 1995.

Apte S., Mahesh K., Moin P. and Oefelein J., Large-eddy simulation of swirling particleladen flows in a coaxial-jet combustor, Int. J. of Multiphase Flow, vol. 29, pp. 13111331, 2003.

Ashforth-Frost S., Jambunathan K. and Whitney C.F., Velocity and turbulence characteristics of a semiconfined orthogonally impinging slot jet, Experimental Thermal and Fluid Science, vol. 14, pp. 60-67, 1997.

Barata J. M., Durao D.F.G., Heitor M.V. and MaGuirk J.J., On the analysis of an impinging jet on ground effects, Exp. Fluids, vol. 15, pp. 117-129, 1993.

Barton, I. E., Computation of particle tracks over a backward-facing step, J. Aerosol Sci., vol. 26, p. 881, 1995.

Basset A. B. , A treatise on hydrodynamics, 2nd ed. New York: Dover: Cambridge: Deighton, Bell, 1961.

Berlemont, A., Desjonqueres, P., Gousbet, G., Particle Lagrangian simulation in turbulent flows., Int. J. Multiphase Flow, vol. 16, pp. 19-34, 1990.

Berrouk A. and Laurence D., Stochastic modeling of aerosol deposition for LES of 90 deg. bend turbulent flow, Intr. J. Heat and Fluid Flow, vol. 29, pp. 1010-1028, 2008.

Berrouk A. S., Laurence D. L., Riley J. J. and Stock D. E., Stochastic modelling of 
inertial particle dispersion by subgrid motion for LES of high Reynolds number pipe flow., J. Turbulence, vol. 8, pp. 1-20, 2007.

Breuer M., Baytekin H. and Matida E., Prediction of aerosol deposition in 90 deg. bends using LES and an effecient Lagrangian tracking method, Aerosol Sci., vol. 37, pp. 14071428, 2006.

Burry, D. and Bergeles, G., Dispersion of particles in anisotropic turbulent flows, Int J Multiphase Flow, vol. 19, pp. 651-664, 1993.

Burwash W., Finlay W. and Matida E., Deposition of particles by a confined impinging jet onto a flat surface at $R e=10000$, Aerosol Sc. and Technol., vol. 40, pp. 147-156, 2006.

Chen H. C. and Patel V. C., Near-wall turbulence models for complex flows including seperation, AIAA, vol. 26, pp. 641-648, 1988.

Chen M. and McLaughlin JB., A new correlation for the aerosol deposition rate in vertical sucts, Collide Interface Sci., vol. 169, pp. 437-455, 1995.

Chen Q. and Ahmadi G., Deposition of particles in a turbulent pipe flow, J. Aerosol Sci., vol. 28, no. 5, pp. 789-796, 1997.

Chen X. Renksizbulut M. and Li X., Interaction of a particle-laden gaseous jet with a confined annular turbulent flow, Part. Part. Syst. Charact., vol. 18, pp. 120-133, 2001.

Chorel F., Kondjoyan and Mirade P., Towrd quantitative CFD prediction of contaminant particle deposition against surfaces in large forced-ventilation food plants, Aerosol Sci. and Tech., vol. 44, pp. 10-28, 2010.

Ciampini D., Spelt J., Papini M. Simulation of interference effects in particle streams following impact with a flat surface Part I. Theory and analysis, Wear, vol. 254, pp.237249, 2003a.

Ciampini D., Spelt J., Papini M. Simulation of interference effects in particle streams following impact with a flat surface Part II. Parametric study and implications for erosion testing and blast cleanin, Wear, vol. 254, pp.250-264, $2003 \mathrm{~b}$.

Colucci D. W. and Viskanta R., Effect of nozzle geometry on local convective heat transfer to a confined impinging air jet, Exp. Therm. Fluid Sci., vol. 13, pp. 71-80, 1996.

Cooper D., Jackson D.C., Launder B.E. Liao G.X., Impinging jet studies for turbulence model assessment-I Flow field experiments, Int. Journal of Heat Mass transfer, vol. 36, pp. 2675-2684, 1993.

Corrsin, S. and Lumley, J., On the equation for a particle in turbulent fluid, Appl. Sci. Res., section A, vol. 6, pp. 114-116, 1956.

Craft T. J. Graham L.J. W. and Launder B. E., Impinging jet studies for turbulence model assessment-II. An examination of the performance of four turbulence models, Int. J. Heat Mass Transfer, vol. 36, pp. 2685-2697, 1993. 
Crowe, C., Sommerfeld, M. and Tsuji, Y., Multiphase flows with droplets and particles.: CRC Press, Boca Raton, 1998.

Crowe, C.T., Gore, R.A. and Troutt, T.R., Particle dispersion by coherent structures in free shear flows, Particulate Science and Technology, vol. 3, pp. 149-158, 1985.

Dano B. P. E., Liburdy J. A. and Kanokjaruvijit, Flow characteristics and heat transfer performances of a semi-confined impinging array of jets: effect of nozzle geometry, Intr. J. Heat and Mass Transfer, vol. 48, pp. 691-701, 2005.

Davies C. N., The rate of deposition of aerosol particles from turbulent flow through ducts, Ann. Occup. Hyg., vol. 8, pp. 239-245, 1965.

DeHaan, W. H., and Finlay, W. H., Predicting extrathoracic deposition from dry powder inhalers, J. Aerosol Sci. 35 , vol. 35, pp. 309-331, 2004.

Dehbi, A. , Prediction of extrathoracic aerosol deposition using RANS-Random Walk and LES approaches., Aerosol Sc. and Technol., vol. 45, pp. 555-569, 2011.

Dosanjh, S and Humphrey, J., The influence of turbulence on erosion by a particle-laden fluid jet., Wear, vol. 102, pp. 309-330, 1985.

Elghobashi S., On predicting particle-laden turbulent flows, Applied Scientific Research, vol. 52, pp. 309-329, 1994.

Fan F. and Ahmadi G., A sublayer model for turbulent deposition of particles in verticalducts with smooth and rough surfaces., Aerosol Science, vol. 24, pp. 45-64, 1993.

Fan Q., Wang X., Zhang H. and Chan C., Large eddy simulation of a horizontal particleladen turbulent planar jet, Computational Mechanics, vol. 27, pp. 128-137, 2001.

Finlay W. H., The mechanics of inhaled pharmaceuitical aerosols, Academic Press, 2001.

Fuchs L. and Hallqvist T., Numerical study of impinging jets with heat transfer-inlet conditions effects, in 47th AIAA Aerospace Sciences Meeting Including The New Horizons Forum and Aerospace Exposition, USA, FL, Orlando, 2009, pp. 1-21.

Fuchs, N. A., The mechanics of aerosols . New York: Dover, 1964.

G'omez-Moreno, F. J., Rosell Llompart, J. F., and Fern'andez de la Mora, J. , Turbulent transition in impactor jets and its effect on impactor resolution, J. Aerosol Sci, vol. 33, pp. 459-476, 2002.

Galletti C., Brunazzi, E. and Tognotti, L., A numerical model for gas flow and droplet motion in wave-plate mist eliminators with drainage channels., Chem. Eng. Sci., vol. 63, pp. 5639-5652, 2008.

Ganic E. N. and K. and Mastanaiah KM, Investigation of droplet deposition from a 
turbulent gas stream, Int. J. Multiphase Flow, vol. 7, pp. 401-422, 1981.

Gao N. and Ewing D., Investigation of the effect of confinement on the heat transfer to round impinging jets exiting a long pipe, Int. J. of Heat and Fluid Flow, vol. 27, pp. 33$41,2006$.

Garimella S. and Nenaydykh, Nozzle-geometry effects in liquid jet impingement heat transfer, Intr. Journal of Heat Mass Transfer, vol. 39, no. 14, pp. 2915-2923, 1996.

Gosman, A. D. and Ioannides, E., Aspects of computer simulation of liquid-fueled combustors, AIAA 19th Aerospace Sci. Meeting, 811981.

Govan A. H., Hewitt G.F. and Ngan C.F., Particle motion in a turbulent pipe flow , Int. J. Multiphase Flow, vol. 15, pp. 471-481, 1989.

Graham, D. I., James, P. W., Turbulent dispersion of particles using eddy interaction models., Int. J. Multiphase Flow, vol. 22, no. 1, pp. 157-175, 1996.

Greenfield C. and Quraini G., Particle deposition in a turbulent boundary layer including the effect of thermophoresis, ASME Fluids Engineering Division Summer Meeting FEDSM97-3902, 1997.

Greenfield C. and Quaraini G., A Lagrangian simulation of particle deposition in a turbulent boundary layer in the presence of thermophoresis, Applied Mathematical Modelling, vol. 22, pp. 759-771, 1998.

Hatta N., Fujimoto H. and Ishii R., Numerical analysis of a gas-particle subsonic jet impinging on a flat plate, ISIJ International, vol. 31, no. 4, pp. 342-349, 1991.

Hattori H. and Nagano Y., Direct numerical simulation of turbulent heat transfer in plane impinging jet, Intr. Journal Heat and Fluid Flow, vol. 25, pp. 749-758, 2004.

He C. and Ahmadi G., Particle deposition in a nearly developed turbulent duct floe with electrophoresis, Aerosol Sci., vol. 30, pp. 739-758, 1999.

Huang G. P. Mujumdar A. S. and Douglas W. J. M., Numerical prediction of fluid flow and heat tarnsfer under a turbulent impinging slot jet with surface motion and crossflow, ASME paper 84-WA/HT-33, 1984.

Hutchinson P., Hewitt G.F. and Dukler A.E., Deposition of liquid or solid dispersions from turbulent gas streams: a stochastic model, Chem. Engng. Sci., vol. 26, pp. 419-439, 1971.

Jambunathan, K., Lai, E., Moss, M. A. and Button, B. L., A review of heat transfer data for single circular jet impingement. Int. J. Heat and Fluid Flow, Vol.13, No.2, June, 1992.

James P., Wang Y., Azzopardi B. and Hughes J., The role of drainage channels in the performance of wave-plate mist eliminators, Trans IChemE, vol. 81, Part A, pp. 639648, 2003.

Jaramillo J. E., Perez-Segarra, Rodrigues I. and Oliva A., Numerical study of plane and 
round impinging jets using RANS models, Numerical Heat Transfer, Part B. , vol. 54, pp. 213-237, 2008.

Jin H. H., LUO K., Fan J. R. and Cen K. F., Large eddy simulation of a particle-laden turbulent plane jet, Journal of Zhejiang University Science, vol. 4, no. 2, pp. 175-180, 2003.

John W., Fritter D. N. and Winklmayr W., Resuspension induced by imacting particles, J. Aerosol Sci., vol. 22, no. 6, pp. 723-736, 1991.

Kallio G. A. and Reeks M. W., A numerical simulation of particle deposition in turbulent boundary layers, Int. J. Multiphase Flow, vol. 15, no. 3, pp. 433-446, 1989.

Kato M. and Launder B.E., The modelling of turbulent flow around stastionary and vibrating square cylinders, UMIST, Ninth symposium on turbulent shear flows 1993.

Kim J., Moin P. and Moser R. D., Turbulent statistics in fully developed channel flow at low Reynolds number, J. Fluid Mech., vol. 177, pp. 133-166, 1987.

Kim, I., Elgobashi, S. and Sirignano, W. A., On the equation for spherical-particle motion: effect of Reynolds and acceleration numbers, Journal of Fluid Mechanics, vol. 367, pp. 221-253, 1998.

Knowles K. and Myszko M., Turbulence measurements in radial wall jet, Exp. Therm. Fluid Sci, vol. 77, pp. 71-78, 1998.

Kraichnan R. H., Diffusion by random velocity field, Phys. Fluid, vol. 11, pp. 43-62, 1970.

Kreplin, H. P. and Eckelmann H., Behaviour of the three fluctuating velocities in the wall region of a turbulent channel flow, phys. Fluids, vol. 22, pp. 1233-1239, 1979.

Kubacki S. and Dick E., Simulation of plane impinging jets with k-w based hybrid RANS/LES models, Intr. J. of Heat and Fluid Flow, vol. 31, pp. 862-878, 2010a.

Kubacki S. and Dick E., Hybrid RANS/LES of low Reynolds number round impinging jets, in ECCOMAS CFD 2010, Lisbon, 2010b, pp. 1-14.

Kurosaki Y., Murasaki T., Satoh I. and Kashiwagi T., Study on heat transfer mechanism of a gas-solid suspension impinging jet(effect of particle sizes and thermal properties), Heat Transfer, vol. 5, pp. 2587-2592, 1986.

Kurosaki, Y., Satoh, I., Kameoka, Y. and Annmo, Y., Mechanisms of heat transfer enhancement around the stagnation point of an impinging air jet laden with solid particles, in 9th Int. Heat Transfer Conference, Jerusalem, 1990, pp. 99-104.

Lange R., Fissan H. and Schmidt-Ott A., A new method for the determination of the interception equivalent diameter, Aerosol Science, vol. 31, pp. 221-231, 2000. 
Lee K. W. and Gieseke J. A., Deposition of particles in turbulent pipe flows, Aerosol Science, vol. 25, pp. 699-709, 1994.

Lei K., Kase K., Oshima N. and Kobayashi T., Solid particle distribution in particleladen turbulent channel flows, Progress in Computational Fluid Dynamics, vol. 8 , pp. 413-423, 2008.

Li D., Fan J., Luo K. and Cen K., Direct numerical simulation of a particle-laden low Reynolds number, International Journal of Multiphase Flow, vol. 37 , pp. 539-554, 2011.

Li, A. and Ahmadi, G. , Deposition of aerosols on surfaces in aturbulent channel flow, Int. J. Eng. Sci., vol. 31, pp. 435-451, 1993.

Li, A. and Ahmadi, G. , Dispersion and deposition of spherical particles from point sources in a turbulent channel flow., Aerosol Sci. Technol., vol. 16, pp. 209-226, 1992.

Li, A., Ahmadi G., Bayer R. and Gaynes M. , Aerosol particle deposition in an obstructed turbulent duct flow., J. Aerosol Sci., vol. 25, pp. 91-112, 1994.

Ling W., Chung JN. and Crowe CT., Direct numerical simulation of the two-way coupled interaction between particles and mixing layer, Proc. R. Soc Lond A, vol. 456, pp. 2931-2955, 2000.

Luo X.Y., Hinton J.S., Liew T.T. and Tan K.K., LES modelling of flow in a simple airway model, Medical Engineering and Physics, vol. 26, pp. 403-413, 2004.

MacInnes, J. M. and Bracco, F. V., Stochastic particle dispersion modeling and the tracer-particle limit., Phys. Fluids, A4, vol. 12, pp. 2809-2824, 1992.

McCoy D.D. and Hanratty T. J., Rate of deposition of droplets in annular two-phase flow, Int. J. Multiphase flow, vol. 3, pp. 319-331, 1977.

Mashayek F. and Pandya R. V. R., Analytical description of particle/droplet-laden turbulent flows, Progress in energy and combustion science, vol. 29, pp. 329-378, 2003.

Matida, E. A., Nishino, K. and Torii, K., Statistical simulation of particle deposition on the wall from turbulent dispersed pipe flow., Int. J. Heat Fluid Flow, vol. 21, pp. 389$402,2000$.

Matida, E.A., Finlay, W.H., Breuer, M. and Lange, C.F., Improving prediction of aerosol deposition in an idealized mouth using Large-Eddy Simulation., J. Aerosol Med., vol. 19, pp. $290-300,2006$.

Maxey, M. R. and Riley, J. J., Equation of motion for a small rigid sphere in a nonuniform flow, Phys. Fluids, vol. 26, pp. 883-889, 1983.

McLaughlin, J. B. , Aerosol particle deposition in numerically simulated channel flow., Phys. Fluids A1, vol. 7, pp. 1211-1224, 1989. 
Mei, R., Velocity fidelity of flow tracer particles, Exp. Fluids, vol. 22, pp. 1-13, 1996.

Menter, F. R., Two-Equation eddy-viscosity turbulence models for engineering applications, AIAA J., vol. 32, pp. 1598-1605, 1994.

Mostafa A. and Mongia H., On the interaction of particles and turbulent fluid flow, Int. J. Heat Mass Tranfer, vol. 31, no. 10, pp. 2063-2075, 1988.

Nijdam J. Guo B., Fletcher D. and Langrish T., Validation of the Lagrangian approach for predicting turbulent dispersion and evaporation of droplets within a spray, Drying Tech., vol. 24, pp. 1373-1379, 2006.

Nishino K., Samada M., Kasuya K, Torii K., Turbulence statistics in the stagnation region of an axisymmetric impinging jet flow, Journal of Heat Fluid Flow, vol. 17, pp. 193-201, 1996.

Obot N. T., Mujumdar A. S. and Douglas W. J. M., Effect of nozzle geometry on impingement heat transfer under a round turbulent jet, American Society of Mechanical Engineers, 79 -WA/HT-53, 1979a.

Ormancey A. and Martinon J., Prediction of particle dispersion in turbulent flows, Phys. Chem. Hydrodyn., vol. 5, p. 229, 1984.

Ounis, H., Ahmadi, G. and McLaughlin, J. B. , Dispersion and deposition of Brownian particles from point sources in a simulated turbulent channel flow., J. Colloid Interface Sci., vol. 143, pp. 266-277, 1991.

Park T. S. and Sung H. J., Development of a near-wall turbulence model and application to jet impingement heat transfer, Intrnational Journal of Heat and Fluid Flow, vol. 22, pp. $10-18,2001$.

Piomelli U., Large-eddy and direct simulation of turbulent flows, in 9e Conference Annuelle de la Societe Canadienne de CFD, Montreal, 2001.

Polat S., Huang A., Mujumdar A. and Douglas W. J., Numerical flow and heat transfer under impinging jets, Annual Review of Numerical Fluid Mechanics and Heat Transfer, vol. 2, pp. 157-197, 1989.

Pope S. B., Turbulent flows. New York: Cambridge university press, 2000.

Quispe J.R. Canales E. and Borquez R., Simulation of turbulent flows in an impingegment dryer by an extended k-epsilon model, Comput. Methods Appl. Mech. Engrg., vol. 190, pp. 625-637, 2000.

Rao N. and Faghri M., Computer modeling of electrical enhancement in fibrous filters, Aerosol Science and Tech., vol. 13, pp. 127-143, 1990.

Rhea S., Bini M., Fairweather M. and Jones W. P., RANS modelling and LES of a single-phase, impinging plane jet, Computers and Chemical Engineering, vol. 33, pp. 
1344-1353, 2009.

Sehmel G. A., Complexities of particle deposition and Re-entrainment in turbulent pipe flow, Aerosol Science, vol. 2, pp. 63-72, 1971.

Sethi, V., and John, W. , Particle impaction patterns from a circular jet, Aerosol Sci. Technol. , vol. 18, no. 1, pp. 1-10, 1993.

Seyedein S. , Hasan M. and Mujumdar A., Modeling of a single confined turbulent slot jet impingement using various k-epsilon turbulence models, Appl. Math. Modelling, vol. 18, pp. 526-537, 1994.

Seyedein S. H., Hasan M. and Mujumdar A. S., Turbulent flow and heat transfer from confined multiple impinging slot jets, Numerical Heat transfer; Part A: Applications, vol. 27, pp. 35-51, 1995.

Shi Y., Ray M. and Mujumdar A., Effects of Prandtl number on impinging jet heat transfer under a semi-confined turbulent slot jet, Int. Comm. Heat Mass Transfer, vol. 29, pp. 929-938, 2002a.

Shi Y., Ray M. and Mujumdar A., Numerical study on the effect of cross-flow on turbulent flow and heat transfer characteristics under normal and oblique semi-confined impinging slot jets, Drying Technology, vol. 21, pp. 1923-1939, 2003 a.

Shi Y., Mujumdar A. and Ray M., Parametric study of heat transfer in turbulent gas-solid flow in multiple impinging jets, Ind. Eng. Chem. Res., vol. 42, pp. 6223-6231, $2003 \mathrm{~b}$.

Shimizu A., Echigo R. and Hasegawa S., Impinging jet heat transfer with gas-solid suspension medium, in 18th Nat.Heat Transfer Conference, San Diego, CA, USA, 1979, pp. 155-160.

Shotorban B., Zhang K. and Mashayek F., Improvement of particle concentration prediction in large-eddy simulation by defiltering, Int. J. Heat and Mass Transfer, vol. 50, pp. 3728-3739, 2007.

Shuen, J. S., Chen, L. D., and Faeth, G. M., Evaluation of a Stochastic model of particle dispersion in a turbulent round jet, AIChE J, vol. 29, pp. 167-170, 1983 a.

Shuen, J. S., Chen, L. D., and Faeth, G. M., Predictions of the structure of turbulent, particle-laden round jets, AIAA, vol. 21, no. 11, pp. 1483-1484, $1983 \mathrm{~b}$.

Sigalla A., Measurements of a skin-friction in a plane turbulent wall jet, Aero. Soc., vol. 62, p. $873,1958$.

Smagorinsky J.S., General circulation experiments with the primitive equation, Monthly Weather Review, vol. 91, pp. 99-164, 1963.

Sommerfield m., Kohnen G. and Ruger M., Some open questions and inconsistencies of Lagrangian particle dispersion models, in 9th Symp. Turbulent Shear Flows, Kyoto, 
1993, pp. 1-6.

Tailland A. and Mathieu J., Jet parietal, Journal of Mecanique, vol. 6, p. 1, 1967.

Tchen C. M., Mean value and correlation problems connected with the motion of small particles in a turbulent field, Delft University, Netherlands, Ph.D Dissertation 1947.

Thomson D. J., Criteria for the selection of stochastic modes of particle trajectories in turbulent flows, J. Fluid Mech., vol. 180, pp. 529-556, 1987.

Tian L. and Ahmadi G. , Particle deposition in turbulent duct flow- comparisons of different model predictions., Aerosol Sci. , vol. 38, pp. 377-397, 2007.

Uijttewaal W. Oliemans R., Particle dispersion and deposition in direct numerical simulation and large eddy simulations of vertical pipe flows, Phys. Fluids, vol. 8, pp. 2590-2604, 1996.

Van Driest E.R., On turbulent flow near a wall, AIAA Journal, vol. 23, pp. 1007-1011, 1956.

Wagner C., Huttl, T. and Sagaut P., Large eddy simulation for acoustics. New York: Campridge University Press, 2007.

Wang S. J. and Mujumdar A., A comparative study of five low Reynolds number kepsilon models for impingement heat transfer, Applied Thermal Engineering, vol. 25, pp. 31-44, 2005.

Wang Y. and James P., The calculation of wave-plate demister effeciencies using numerical simulation of the flow field and droplet motion, Trans IChemE, vol. 76, Part A, pp. 980-985, 1998.

Wang, L. P., and Stock, D. E., Stochastic trajectory models for turbulent diffusion: Monte Carlo Process Versus Markov Chains, Atmosphere Envir. , vol. 26A, no. 9, pp. 1599-1607, 1992.

Wang, Y., James, P.W. , Assessment of an eddy-interaction model and its refinements using predictions of droplet deposition in a wave-plate demister., Chemical Engineering Research \& Design, vol. 77, pp. 692-698, 1999a.

Wang, Y., and James, P.W. , On the effect of anisotropy on the turbulent dispersion and deposition of small particles, Int. J. Multiphase Flow, vol. 25, pp. 551-558, 1999 b.

Wang., Q., and Squires, K. D., Large eddy simulation of particle deposition in a vertical turbulent channel flow., Int. J. Multiphase flow, vol. 22, no. 4, pp. 667-683, 1996.

Wilcox D.C., Turbulence modeling for CFD , 2nd ed.: DCW Industries Inc., 1998.

Wilcox D.C., Turbulence modeling for CFD , 3rd ed.: DCW Industries Inc., 2006. 
Williams I. and Hedley A., Deposition of aerosols from turbulent pipe flow, Faraday Symp. Chem. Soc., vol. 7, pp. 162-175, 1973.

Wood, N. B. , A Simple method for the calculation of turbulent deposition to smooth and rough surfaces., J. Aerosol Sci., vol. 12, no. 3, pp. 275-290, 1981.

Yeh F. and Lei U., On the motion of small particles in a homogeneous isotropic turbulent Flow, Phys. Fluid, vol. 3, no. 11, pp. 2571-2586, 1991.

Yokomine T., Shimizu A., Hasegawa S. and Kawamura T., Stochastic simulation of gas-solid suspension flows by means of Reynolds stress rurbulence model, in ASME/JSME Thermal Engineering Proceedings, 1991, pp. 81-88.

Yokomine T., Shimizu A., Saitoh A. and Higa K., Heat transfer of multiple impinging jets with gas-solid suspensions, Experimental Thermal and Fluid Science, vol. 26, pp. 617-626, 2002.

Yoshida H., Suenaga K. and Echigo, Turbulence structure and heat transfer of a twodimensional impinging jet with gas-solid suspensions, Int. J. Heat Mass Transfer, vol. 33, pp. 859-867, 1990.

Yoshida H., Suenaga K. and Echigo, R., Turbulence structure and heat transfer of a two-dimensional impinging jet with gas-solid suspensions, in National Heat Transfer, Houston, TX, USA, 1988, pp. 461-467.

Yuu S, Yasukouchi N and Hirosawa Y., Particle turbulent diffusion in a dust laden round jet., AIChE Journal, vol. 24, pp. 509-519, 1978.

Yuu S., Ueno T.and Umekage T, Numerical simulation of the high Reynolds number slit nozzle gas-particle jet using subgrid-scale coupling large eddy simulation, Chemical Engineering Science, vol. 56, pp. 4293-4307, 2001.

Zamora B. and Kaiser A. S., Comparative efficiency evaluations of four types of cooling tower drift eliminator, by numerical investigation., Chem. Eng. Sci. , vol. 66, pp. 1232-1245, 2011.

Zhang P., Xu G.H., Fu X. and Li C.R., Confined jet impingement of liquid nitrogen onto different heat transfer surfaces, Cryogenics, vol. 51, pp. 300-308, 2011.

Zhang Y., Finlay. W. and Matida E., Particle deposition measurements and numerical simulation in a highly idealized mouth-throat, Aerosol Sci., vol. 35, pp. 789-803, 2004.

Zhao J., Jin B. and Zhong Z., Study of the separation effeciency of a demister vane with response surface methodology, Hazardous Materials, vol. 147, pp. 363-369, 2007.

Zhou Q. and Leschziner M.A., A time-correlated stochastic model for particle dispersion in anisotropic turbulence, in 8th Turbulent Shear Flow Symposium, Munich, 1991, pp. 1-6. 
Zhou, Q. and Leschziner, M. A., Modelling particle dispersion in anisotropic turbulence, ECCOMAS 96, John Wiley, pp. 577-583, 1996. 


\section{Appendices}

\section{Appendix A RANS models}

\section{A.1 Shear stress transport (SST) model}

The SST model can be developed using the original $\mathrm{k}-\omega$ model as follows

$$
\begin{aligned}
& \frac{\partial k}{\partial t}+U_{j} \frac{\partial k}{\partial x_{j}}=\frac{\partial}{\partial x_{j}}\left[\left(v+\sigma_{k 1} v_{T}\right) \frac{\partial k}{\partial x_{j}}\right]+P_{k}-\beta^{\prime} \omega k \\
& \frac{\partial \omega}{\partial t}+U_{j} \frac{\partial \omega}{\partial x_{j}}=\frac{\partial}{\partial x_{j}}\left[\left(v+\sigma_{\omega 1} v_{T}\right) \frac{\partial \omega}{\partial x_{j}}\right]+\alpha_{1} \frac{\omega}{k} P_{k}-\beta_{1} \omega^{2}
\end{aligned}
$$

The SST model is obtained by developing the transformed $\mathrm{k}-\varepsilon$ model as

$$
\begin{aligned}
& \frac{\partial k}{\partial t}+U_{j} \frac{\partial k}{\partial x_{j}}=\frac{\partial}{\partial x_{j}}\left[\left(v+\sigma_{k 2} v_{T}\right) \frac{\partial k}{\partial x_{j}}\right]+P_{k}-\beta^{\prime} k \omega \\
& \frac{\partial \omega}{\partial t}+U_{j} \frac{\partial \omega}{\partial x_{j}}=\frac{\partial}{\partial x_{j}}\left[\left(v+\sigma_{\omega 2} v_{T}\right) \frac{\partial \omega}{\partial x_{j}}\right]+\alpha_{2} \frac{\omega}{k} P_{k}-\beta_{2} \omega^{2}+2 \sigma_{\omega 2} \frac{1}{\omega} \frac{\partial k}{\partial x_{j}} \frac{\partial \omega}{\partial x_{j}}
\end{aligned}
$$

The model constants are as follows

$$
\begin{aligned}
& \sigma_{k 1}=0.5, \quad \beta^{\prime}=0.09, \quad \sigma_{\omega 1}=0.5, \quad \alpha_{1}=\frac{5}{9}, \quad \beta_{1}=0.075 \\
& \sigma_{k 2}=1, \quad \sigma_{\omega 2}=0.856, \quad \alpha_{2}=0.44, \quad \beta_{2}=0.0828
\end{aligned}
$$

The production term $P_{k}$ is formulated using a limiter as follows

$$
P_{k}=\min \left(v_{T} 2 \mathrm{~S}_{\mathrm{ij}} \frac{\partial U_{i}}{\partial x_{j}}, 10 \rho \varepsilon\right)
$$

where $v_{T}=\frac{k}{\omega}$ and $S_{i j}$ the mean strain rate tensor is given as

$$
S_{i j}=\frac{1}{2}\left(\frac{\partial U_{i}}{\partial x_{j}}+\frac{\partial U_{j}}{\partial x_{i}}\right)
$$


The SST model constants are determined from the following relation

$\phi=F_{1} \phi_{1}+\left(1-F_{1}\right) \phi_{2}$

$\phi_{1}$ represents any constant in the k- $\omega$ model and $\phi_{2}$ any constant in the k- $\varepsilon$ model. $F_{1}$ is a blending function given as

$F_{1}=\tanh \left(\arg _{1}^{4}\right)$

with

$\arg _{1}=\min \left[\max \left(\frac{k^{\frac{1}{2}}}{\beta^{\prime} \omega \mathrm{y}}, \frac{500 v}{\mathrm{y}^{2} \omega}\right), \frac{4 \rho k}{C D_{k W} \sigma_{\omega 2} y^{2}}\right]$

where $\mathrm{y}$ is the distance to the nearest wall, $v$ is the kinematic viscosity and

$C D_{k \omega}=\max \left(2 \rho \frac{1}{\sigma_{\omega 2} \omega} \frac{\partial k}{\partial x_{i}} \frac{\partial \omega}{\partial x_{i}}, 1.0 \times 10^{-10}\right)$

The proper transport behavior is obtained by a limiter to the formulation of the eddy viscosity:

$v_{T}=\frac{0.31 k}{\max \left(0.31 \omega, \Omega F_{2}\right)}$

where $\Omega$ is the absolute value of vorticity, and

$$
F_{2}=\tanh \left(\arg _{2}^{2}\right)
$$

with

$\arg _{2}=\max \left(\frac{2 k^{\frac{1}{2}}}{\beta^{\prime} \omega \mathrm{y}}, \frac{500 v}{y^{2} \omega}\right)$

$F_{2}$ is a blending function similar to $F_{1}$. The formulation of the blending function is based on the distance to the nearest surface and on the flow variables. 
The SST model makes use of the production limiter of Kato and Launder (1993), which is provided as an option in CFX 12.0, to limit the excessive generation of turbulence energy in the vicinity of the stagnation point. When the standard eddy viscosity approximation is used to solve for the Reynolds stress in the turbulence equations, the production term is given as

$P_{k}=\mu_{t} S^{2}$

where $S$ is the magnitude of the strain rate $S=\sqrt{2 S_{i j} S_{i j}}$. However, the very high levels of strain rate $S$ in the production term lead to excessive turbulence kinetic energy. Therefore, Kato and Launder (1993) suggested the following replacement for the production term

$P_{k}=\mu_{t} S \Omega$

Where the magnitude of the vorticity rate is $\Omega=\sqrt{2 \Omega_{i j} \Omega_{i j}}$ and the vorticity tensor $\Omega_{i j}$ is given as

$$
\Omega_{i j}=\frac{1}{2}\left(\frac{\partial U_{i}}{\partial x_{j}}-\frac{\partial U_{j}}{\partial x_{i}}\right)
$$

This limiter is helpful in reducing the excessive levels of turbulence kinetic energy near the stagnation point since the deformation near this point is very nearly irrotational, i.e., the vorticity rate $\Omega$ is very small.

\section{A.2 Reynolds stress model (RSM) model}

For the RSM models used in the present study, the following Reynolds stress equations can be written as

$$
\frac{\partial \rho \overline{u_{i} u_{j}}}{\partial t}+\frac{\partial}{\partial x_{k}}\left(U_{k} \rho \overline{u_{i} u_{j}}\right)=P_{i j}+\phi_{i j}-\frac{2}{3} \beta^{\prime} \rho \omega k \delta_{i j}+\frac{\partial}{\partial x_{k}}\left[\left(\mu+\frac{\mu_{t}}{\sigma_{k}}\right) \frac{\partial \overline{u_{i} u_{j}}}{\partial x_{k}}\right]
$$


such that the pressure strain term is given as

$\phi_{i j}=\beta^{\prime} C_{1} \rho \omega\left(-\overline{u_{i} u_{j}}+\frac{2}{3} k \delta_{i j}\right)-\hat{\alpha}\left(P_{i j}-\frac{2}{3} P \delta_{i j}\right)-\hat{\beta}\left(D_{i j}-\frac{2}{3} P \delta_{i j}\right)-\hat{\gamma} \rho k\left(S_{i j}-\frac{1}{3} S_{k k} \delta_{i j}\right)$

where $P=0.5 P_{k k}$, and the production tensor of the Reynolds stresses term is

$P_{i j}=-\rho \overline{u_{i} u_{k}} \frac{\partial U_{j}}{\partial x_{k}}-\rho \overline{u_{j} u_{k}} \frac{\partial U_{i}}{\partial x_{k}}$

and the diffusion of the Reynolds stress in the pressure strain term is given as

$D_{i j}=-\rho \overline{u_{i} u_{k}} \frac{\partial U_{k}}{\partial x_{j}}-\rho \overline{u_{j} u_{k}} \frac{\partial U_{k}}{\partial x_{i}}$

In addition, $\mu_{t}=\rho \frac{k}{\omega}$

where the constants are given as

$\beta^{\prime}=0.09, \sigma_{k}=2, \hat{\alpha}=\frac{8+C_{2}}{11}, \hat{\beta}=\frac{8 C_{2}-2}{11}, \hat{\gamma}=\frac{60 C_{2}-4}{55}, C_{1}=1.8, C_{2}=0.52$.

The transport equation for $\omega$ can be obtained from either the RSM- $\omega$ or RSM-BSL.

\section{RSM- $\omega$ model}

The RSM- $\omega$ model uses the following equation (Wilcox, 1998) for $\omega$

$\frac{\partial(\rho \omega)}{\partial t}+\frac{\partial\left(U_{k} \rho \omega\right)}{\partial x_{k}}=\alpha \rho \frac{\omega}{k} u_{i} u_{j} \frac{\partial U_{i}}{\partial x_{j}}-\beta \rho \omega^{2}+\frac{\partial}{\partial x_{k}}\left[\left(\mu+\frac{\mu_{t}}{\sigma}\right) \frac{\partial \omega}{\partial x_{k}}\right]$

where the constants are

$\sigma=2$

$\beta=0.075$

$\alpha=\frac{5}{9}$

\section{RSM-BSL model}


In the RSM-BSL model, the $\omega$-equation is adopted in a similar manner as the SST model (Menter, 1994). However, the coefficients of the equation are blended between values from the $\omega$-based model constants and the $\varepsilon$-based model constants transformed to the $\omega$ formulation. The new $\omega$-equation is written as

$$
\frac{\partial(\rho \omega)}{\partial t}+\frac{\partial\left(U_{k} \rho \omega\right)}{\partial x_{k}}=\alpha_{3} \frac{\omega}{k} u_{i} u_{j} \frac{\partial U_{i}}{\partial x_{j}}-\beta_{3} \rho \omega^{2}+\frac{\partial}{\partial x_{k}}\left[\left(\mu+\frac{\mu_{t}}{\sigma_{\omega 3}}\right) \frac{\partial \omega}{\partial x_{k}}\right]+\left(1-F_{1}\right) \frac{2 \rho}{\sigma_{2} \omega} \frac{\partial k}{\partial x_{k}} \frac{\partial \omega}{\partial x_{k}}
$$

The coefficients of $\alpha_{3}, \beta_{3}, \sigma_{\omega 3}$ are obtained by blending between set1 and set2 constants via

$\phi_{3}=F_{1} \cdot \phi_{1}+\left(1-F_{1}\right) \phi_{2}$

where $F_{1}=\tanh \left(\arg ^{4}\right), \phi_{1}$ and $\phi_{2}$ represent any constant from $\omega$-zone and $\varepsilon$-zone, respectively.

$F_{1}=\tanh \left(\arg ^{4}\right)$

where $\quad \arg =\min \left[\max \left(\frac{k^{\frac{1}{2}}}{\beta^{\prime} \omega \mathrm{y}}, \frac{500 v}{\mathrm{y}^{2} \omega}\right), \frac{4 \rho k}{C D_{k W} \sigma_{k-\varepsilon} y^{2}}\right]$, and the cross-diffusion is given as $C D_{k \omega}=\max \left(2 \rho \frac{1}{\sigma_{k-\varepsilon} \omega} \frac{\partial k}{\partial x_{j}} \frac{\partial \omega}{\partial x_{j}}, 1.0 \times 10^{-10}\right)$.

Set $1(\omega$ zone $)$

$$
\begin{aligned}
& \sigma_{1}=2 \\
& \beta_{1}=0.075 \\
& \alpha_{1}=0.553
\end{aligned}
$$

Set 2 ( $\varepsilon$ zone) 


$$
\begin{aligned}
& \sigma_{2}=0.856 \\
& \beta_{2}=0.0828 \\
& \alpha_{2}=0.44 \\
& \sigma_{k-\varepsilon}=\frac{1}{0.856}
\end{aligned}
$$

With this blending, the RSM-BSL combines the best features of the $\varepsilon$-based model, which predicts the free shear layers, and the $\omega$-based model, which accurately predicts the near-wall region. This makes the RSM-BSL model more suited to wall bounded flows. 


\section{Appendix B Smagorinsky Subgrid Scale Modeling}

Smagorinsky subgrid scale model (Smagorinsky, 1963) is the most commonly used model in LES to date. It is given by the equation

$\tau_{i j}-\frac{1}{3} \tau_{k k} \delta_{i j}=-2 v_{T} \bar{S}_{i j}=-v_{T}\left(\frac{\partial \bar{U}_{i}}{\partial x_{j}}+\frac{\partial \bar{U}_{j}}{\partial x_{i}}\right)$

Where $v_{T}$ is referred to as the eddy viscosity, and the large scale strain rate tensor is given as

$\bar{S}_{i j}=-\frac{1}{2}\left(\frac{\partial \bar{U}_{i}}{\partial x_{j}}+\frac{\partial \bar{U}_{j}}{\partial x_{i}}\right)$

The Smagorinsky model is an eddy viscosity model in which the subgrid scale stress $\tau_{i j}$ is related to the large scale strain rate tensor $\bar{S}_{i j}$. It is known as an eddy viscosity model since it assumes that the small eddies remove the energy from the flow through the dissipation process. This model is based on the hypothesis (Piomelli, 2001) that the energy is produced at the large scale level and transported to smaller and smaller scales (smaller eddies) where the viscous dissipation occurs. Under this hypothesis, the viscous dissipation of SGS energy is written as

$\varepsilon_{v}=-\tau_{i j} \bar{S}_{i j}$

If the viscous dissipation is modeled as

$$
\varepsilon_{v} \sim \frac{q_{S G S}^{3}}{\ell}
$$

where $q_{S G S}$ and $\ell$ are, respectively, velocity and length scales, and then Equation (B.1) is substituted in Equation (B.3) such that

$v_{T} \sim \ell q_{S G S}$ 
the following relation can be obtained as

$q_{S G S} \sim \ell|\bar{S}|$

where $|\bar{S}|=\left(2 \bar{S}_{i j} \bar{S}_{i j}\right)^{0.5}$ is referred to as the magnitude of the strain rate tensor. Making the simplification $\ell \sim \Delta$ ( $\Delta$ is the filter width), the eddy viscosity in the Smagorinsky model can be written as

$v_{T}=\left(C_{S} \Delta\right)^{2}|\bar{S}|$

where $C_{S}$ is the Smagorinsky constant. Also, the Smagorinsky model makes use of the Van Driest (1956) wall damping to damp out the eddy viscosity close to the walls. (Wagner et al., 2007). Therefore, by the introduction of the Van Driest damping to account for the reduced growth of the small scales near the walls, the eddy viscosity is then rewritten in the following form

$v_{T}=\left(C_{S} \Delta D_{\text {wall }}\right)^{2}|\bar{S}|$

where the Van Driest wall damping $D_{\text {wall }}$ is $D_{\text {wall }}=1-e^{\frac{-y^{+}}{25}}$. 


\section{Appendix C Grid convergence for computational domains based on Cooper's experiment}

Similar to grid convergence analysis that was conducted for computational domains based on Burwash's et al. (2006) experiment; a grid convergence analysis for the computational domains based on Cooper's et al. (1993) experiment was conducted. The analysis was performed using RANS with the SST model by considering three different grid sizes of $1,310,784,2,422,679$ and $4,537,168$ nodes for $L / D=2$. The grid size for the $L / D=6$ case was constructed based on the adequate grid size chosen for $L / D=2$. The velocity distribution and the kinetic energy along the jet centerline axis and along a horizontal line $(y=0.1 D)$ parallel to the impingement surface were used to test the grid convergence for the flow field.

\section{C.1 Velocity distribution}

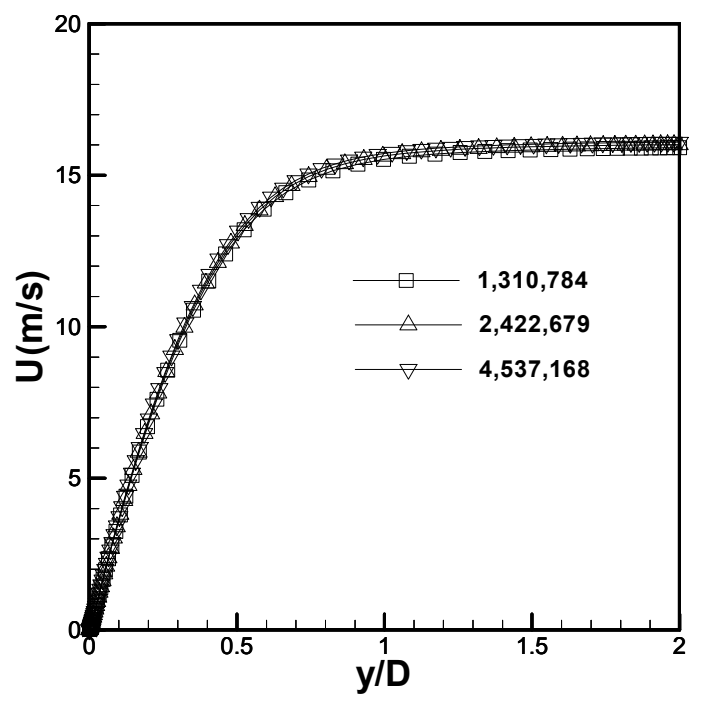

Figure C.1. Velocity magnitude along the centerline axis. 
Figure C.1 and Figure C.2 show, respectively, the velocity magnitude along the centerline axis as a function of $y / D$ and the velocity along a line parallel to the impingement wall ( $y$ $=0.1 D)$ as a function of $x / D$. Figure C.1 indicates that the velocities for the three grid sizes decay with the same trend as the flow approaches the impingement wall. Figure C.2 shows that the velocity profiles for the three grid sizes collapse on each other showing a symmetric behavior around the centerline axis.

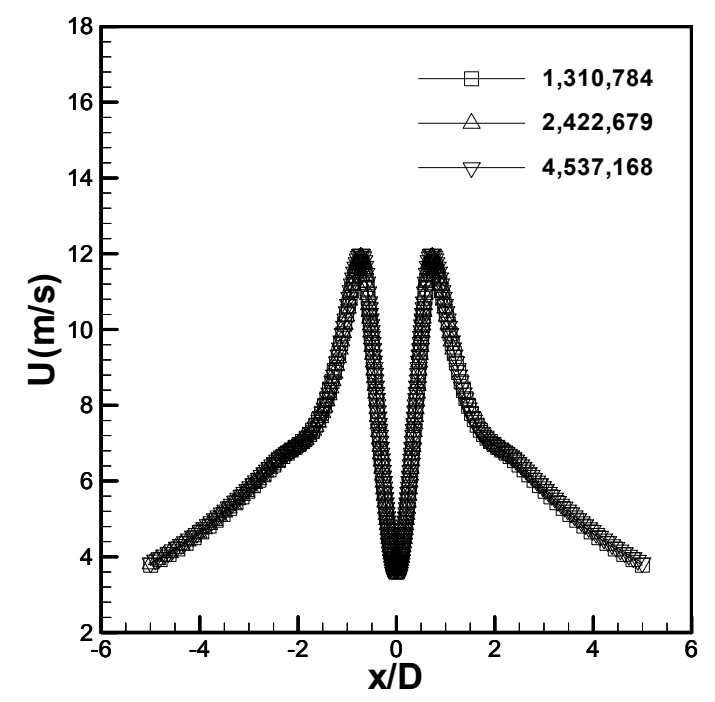

Figure C.2. Velocity along a line parallel to the impingement wall.

\section{C.2 Kinetic energy}

Figure C.3 and Figure C.4 show, respectively, the kinetic energy profiles along the centerline axis as a function of $y / D$ and the kinetic energy profiles along a line $(y=0.1 D)$ parallel to the impingement wall versus $x / D$. As can be seen from Figure C.3, the kinetic energy for the three grid sizes is increasing with the same trend before decaying sharply very close to the wall. Also, in Figure C.4, it can be noted that the kinetic energy profiles for the three grids are identical to each other and symmetric around the centerline axis. 


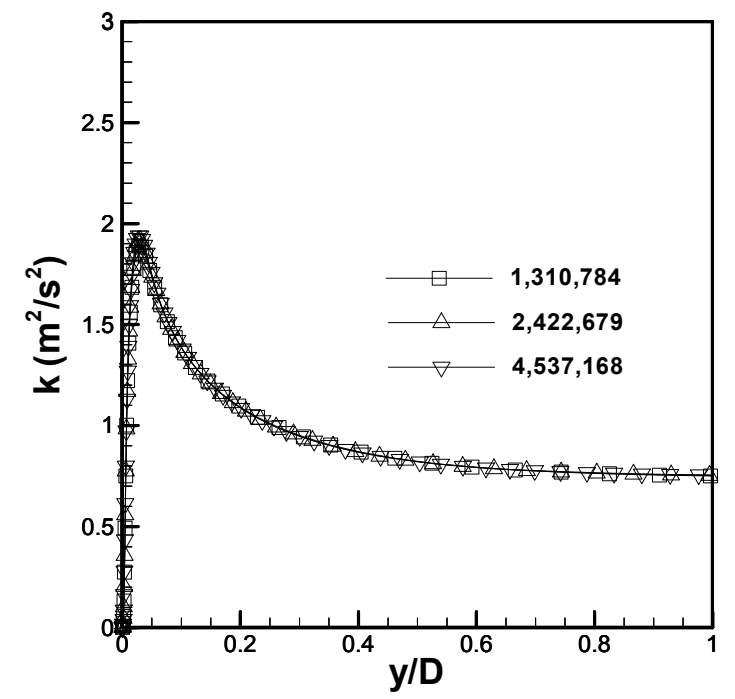

Figure C.3. Kinetic energy profile along the centerline axis.

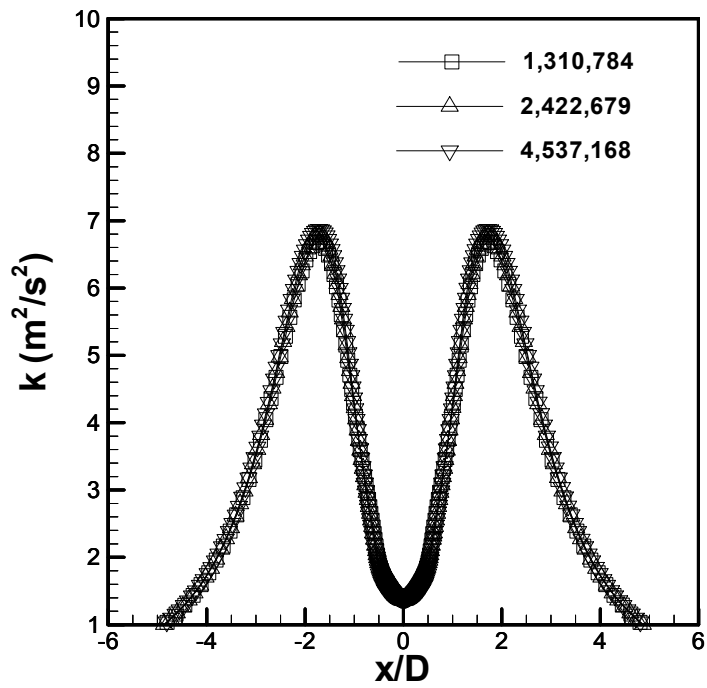

Figure C.4. Kinetic energy profiles along a line parallel to the impingement wall.

From the grid convergence analysis, it can be seen that a number of 2,422,679 nodes is sufficient for accurate results. This finer grid would provide better results in terms of accuracy. Therefore, this grid was used to perform the fluid flow simulations based on Cooper's et al. (1993) experiment. 